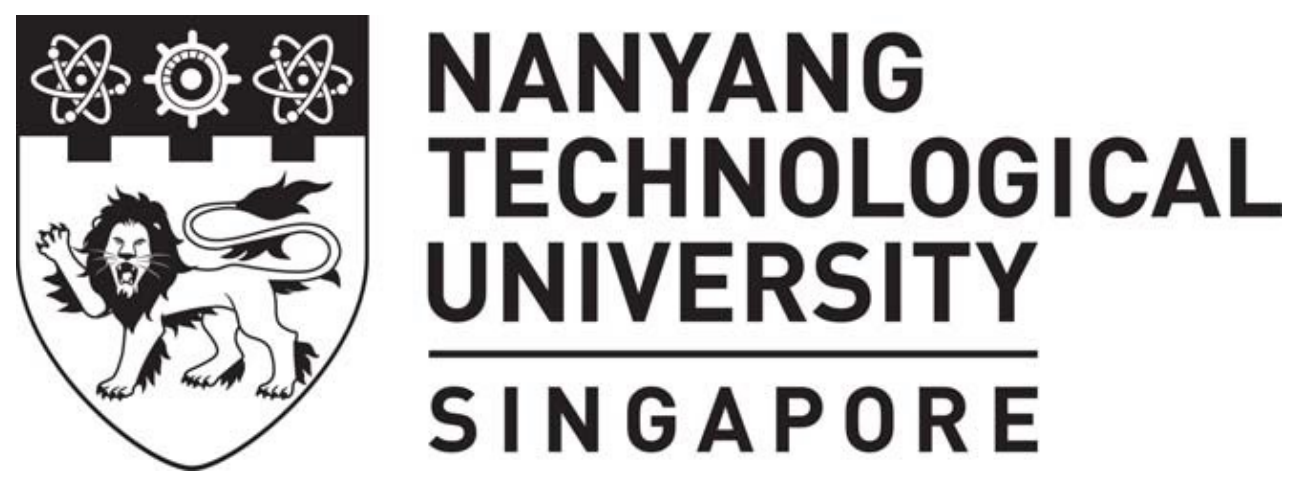

\title{
MICRO AND NANOTECHNOLOGY FOR REGENERATIVE MEDICINE: CELL TRACKING AND ENGINEERING
}

SHIYING LIU

SCHOOL OF CHEMICAL AND BIOMEDICAL ENGINEERING 2017 


\section{MICRO AND NANOTECHNOLOGY FOR REGENERATIVE MEDICINE: CELL TRACKING AND ENGINEERING}

SHIYING LIU

School of Chemical and Biomedical Engineering

A thesis submitted to the Nanyang Technological University in fulfilment of the requirement for the degree of Doctor of Philosophy 2017 


\section{ACKNOWLEDGEMENTS}

At the beginning of the thesis, I would like to appreciate my two supervisors Prof Yuejun Kang and Chenjie Xu most sincerely. They are always very patient, considerate and enthusiastic to provide help whenever I have some problems and difficulties. Thank them for teaching me and sharing with me their precious experiences for research project design, problem solving skills, scientific writing and presentation, as well as collaboration with others.

Besides my supervisor, I also want to thank my collaborators including Prof Bin Cao, Prof Milan Mrksich, Dr. Zhigang Xu, Dr. Yingdan Zhang, Dr. Sreejith Sivaramapanicker for their help and support during my researches.

Special thanks to my group members in the two groups, including Dr. Chuah Yon Jin, Dr.Nishanth V Menon, Li Min Tay, Raditya Anggara, Peng Xue, Yafeng Wu, Yingnan Wu, Dang Tran Chien, Dr. David Yeo, Dr. Keming Xu, Dr. Min Wang, Dr. Hao Chang, Christian Wiraja, Mingyue Cui, Yiyuan Han, Mengjia Zheng, Duong Phan Khanh, Daniel Lio, Huseyin Enis Karahan, Zayim Razina S/O Seei Syed, and so on.

Finally, I want to thank my parents, sister and husband for their endless support and contribution and my friends for their encouragement and comfort. 


\section{BIOGRAPHY}

Shiying Liu graduated from Xiamen University with double Bachelor Degree in Biochemical Engineering and Mathematics \& Applied Mathematics in 2010 in China. Then she was recommended to Institute of Process Engineering, University of Chinese Academy of Sciences without examination and obtained a Master Degree in Biochemical Engineering in 2013. She joined as a $\mathrm{PhD}$ student in School of Chemical and Biomedical Engineering, Nanyang Technological University in 2013. She is very interested in addressing biological problems by micro- and nanotechnology, including four directions. The first one was to track mesenchymal stem cell differentiation into adipocytes, osteocytes, and chondrocytes for a long term by photostable polymeric fluorescent nanoparticles. Secondly, $\mathrm{pH}$ and glutathione responsive drug delivery systems and multi-adjuvant whole cell tumor vaccine were developed for anti-cancer chemotherapeutic and immunotherapy. In addition, she also developed a facile and gentle strategy to load hydrophilic molecules into polymeric microneedles by swelling for abnormal scar treatment. Last but not least, microcontact printing technique was used for patterning single cells with desired sizes and shapes to investigate nanoparticle labeling efficiency and transfer between cells. 


\section{LIST OF PUBLICATIONS}

$\left({ }^{\dagger}\right.$ - co-first authors, ${ }^{*}$ - corresponding author)

1. Shi-Ying Liu ${ }^{\dagger}$, Wei Wei ${ }^{\dagger}$, Hua Yue, De-Zhi Ni, Zhan-Guo Yue, Shuang Wang, Qiang Fu, Yue-Qi Wang, Guang-Hui Ma*, Zhi-Guo Su* Nanoparticles-based multi-adjuvant whole cell tumor vaccine for cancer immunotherapy. Biomaterials, 2013, 34, 8291-8300. $($ IF $=8.978)$

2. Shuo Huang ${ }^{\dagger}$, $\underline{\text { Shiying Liu }}^{\dagger}$, Kai Wang, Cangjie Yang, Yimin Luo, Yingdan Zhang, Bin Cao, Yuejun Kang ${ }^{*}$, Mingfeng Wang ${ }^{*}$. Highly fluorescent and bioresorbable polymeric nanoparticles with enhanced photostability for cell imaging. Nanoscale, 2015, 7, 889-895. (IF= 7.915)

3. Shiying Liu ${ }^{\dagger}$, Li Min Tay ${ }^{\dagger}$, Raditya Anggara, Yon Jin Chuah, Yuejun Kang* ${ }^{*}$ Long-term tracking mesenchymal stem cell differentiation with photostable fluorescent nanoparticles. ACS Applied Materials \& Interfaces, 2016, 8, 11925-11933. (IF= 7.332)

4. Shiying Liu, David C Yeo, Christian Wiraja, Hong Liang Tey, Milan Mrksich, Chenjie Xu*. Peptide delivery with poly(ethylene glycol) diacrylate microneedles through swelling effect. Bioengineering \& Translational Medicine, 2017, 2, 258-267.

5. Shiying Liu, Pradeep Bugga, Milan Mrksich, Chenjie Xu* . Nanoparticle labelling transfer between single cells. (in preparation)

6. Shiying Liu, Nishanth V.M., Sreejith S., Kang Yuejun* . Alleneamide-thiol site specific labeling of CD44 vector for selective cancer bioimaging. (in preparation)

7. Zhigang $\mathrm{Xu}^{\dagger}$, $\underline{\text { Shiying Liu }}^{\dagger}$, Yuejun Kang ${ }^{*}$, Mingfeng Wang ${ }^{*}$. Glutathioneand $\mathrm{pH}$-responsive nonporous silica prodrug nanoparticles for controlled release and cancer therapy. Nanoscale, $2015,7, \mathbf{5 8 5 9 - 5 8 6 8}$. $($ IF $=7.915)$

8. Zhigang $\mathrm{Xu}^{\dagger}$, $\underline{\text { Shiying Liu }}^{\dagger}$, Yuejun Kang ${ }^{*}$, Mingfeng Wang ${ }^{*}$. Glutathioneresponsive polymeric micelles formed by a biodegradable amphiphilic triblock copolymer for anticancer drug delivery and controlled release. ACS Biomaterials Science \& Engineering, 2015, 1, 585-592. 
9. Xiaochun Hou, Shiying Liu, Min Wang, Christian Wiraja, Wei Huang, Peggy Chan, Timothy Tan, Chenjie $\mathrm{Xu}^{*}$. Layer-by-layer 3D constructs of fibroblasts in hydrogel for examining transdermal penetration capability of nanoparticles. Journal of Laboratory Automation, 2016, 1-7. $(\mathrm{IF}=1.297)$

10. Zhigang $\mathrm{Xu}$, Shiying Liu, Hui Liu, Cangjie Yang, Yuejun Kang, Mingfeng Wang*. Unimolecular micelles of amphiphilic cyclodextrin-core star-like block copolymers for anticancer drug delivery. Chemical Communications, 2015, 51, 15768-15771. (IF= 6.628)

11. Wei Wei, Pi-Ping Lv, Xiao-Ming Chen, Zhan-Guo Yue, Qiang Fu, ShiYing Liu, Hua Yue, Guang-Hui Ma* . Codelivery of mTERT siRNA and paclitaxel by chitosan-based nanoparticles promoted synergistic tumor suppression. Biomaterials, 2013, 34, 3912-3923. (IF= 8.978)

12. Lifeng Wang, Keming Xu, Xiaochun Hou, Yiyuan Han, Shiying Liu, Christian Wiraja, Cangjie Yang, Jun Yang, Mingfeng Wang, Xiaochen Dong*, Wei Huang", and Chenjie ${ }^{*}{ }^{*}$. Fluorescent Poly (glycerol-cosebacate) acrylate nanoparticles for stem cell labeling and longitudinal tracking. ACS Applied Materials \& Interfaces, 2017, 9, 9528-9538. (IF= 7.332)

13. Sreejith S., Nishanth V.M., Yue Wang, Hrishikesh Joshi, Shiying Liu, Kok Chan Chong, Yuejun Kang, Handong Sun and Mihaiela C. Stuparu* All-organic luminescent nanodots from corannulene and cyclodextrin nanoassembly: continuous-flow synthesis, non-linear optical properties, and bioimaging applications. Materials Chemistry Frontiers. (Cover)

14. Zhigang Xu, Peng Xue, Yong-E Gao, Shiying Liu, Xiaoxiao Shi, Meili Hou, Yuejun Kang*. pH-responsive polymeric micelles based on poly (ethyleneglycol)-b-poly (2-(diisopropylamino) ethyl methacrylate) block copolymer for enhanced intracellular release of anticancer drugs. Journal of Colloid and Interface Science, 2017, 490, 511-519. ( IF= 3.758) 


\section{CONFERENCE PROCEEDINGS}

1. Poster presentation. Highly fluorescent and bioresorbable polymeric nanoparticles with enhanced photostability for cell imaging. Biomedical Engineering Society 8th Scientific Meeting, Singapore, 17th May, 2014.

2. Oral presentation. Long-term tracking mesenchymal stem cell differentiation with photostable fluorescent nanoparticles. 6th International Conference on Mechanics of Biomaterials and Tissues, Hawaii, USA, 6-10 Dec, 2015.

3. Poster presentation. Peptide delivery with poly(ethylene glycol) diacrylate microneedles through swelling effect. School of Chemical and Biomedical Engineering PhD Symposium, Singapore, 21-22 Mar, 2017.

4. Oral presentation. Peptide delivery with poly(ethylene glycol) diacrylate microneedles through swelling effect. 9th International Conference on Materials for Advanced Technologies, Singapore, 18-23 Jun, 2017. 

AWARDS
1. National Encouragement Scholarship (2007)
2. Third prize in Xiamen University Physics Contest (2008)
3. University first grade scholarship (2008)
4. University first grade scholarship and Wu Simin Scholarship (2009)
5. Third prize in National Challenge Cup Shanghai Expo Special Contest (2009)
6. University second grade scholarship (2010)
7. Awarded "Merit Student" (2007, 2008, 2009, 2010)
8. Awarded "Excellent Graduate" (2010)
9. NTU Research Scholarship (2013-2017) 


\section{TABLE OF CONTENTS}

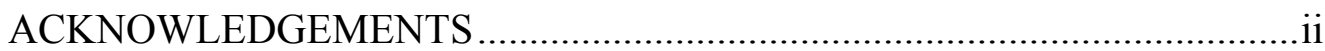

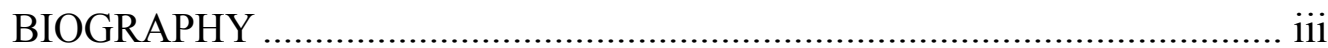

LIST OF PUBLICATIONS .....................................................................

CONFERENCE PROCEEDINGS .............................................................. vi

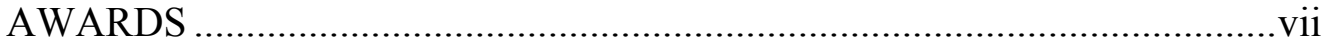

TABLE OF CONTENTS .......................................................................... viii

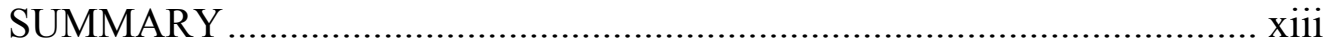

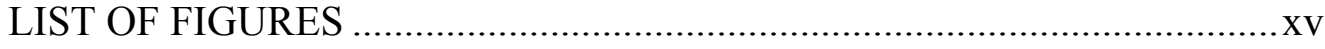

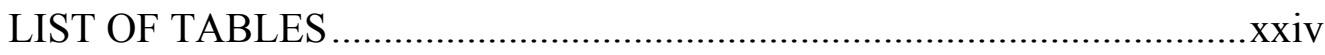

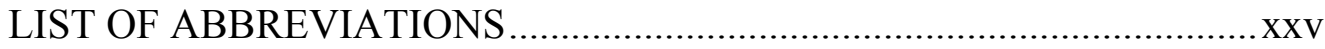

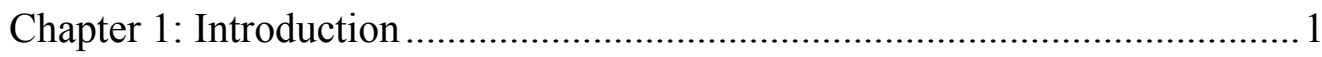

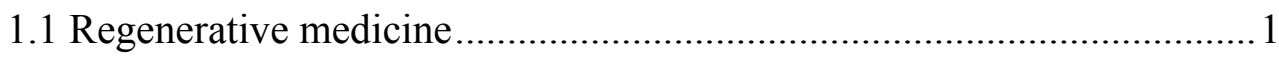

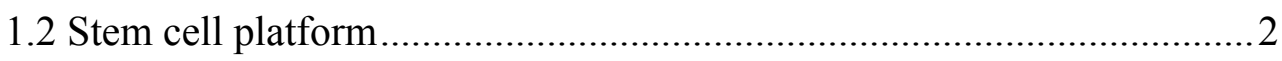

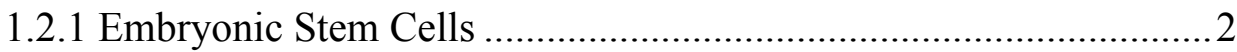

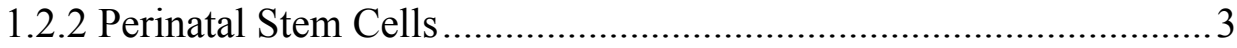

1.2.3 Engineered stem cells ............................................................ 4

1.2.4 Mesenchymal stem cells .......................................................... 4

1.3 Mesenchymal stem cell tracking.................................................. 6

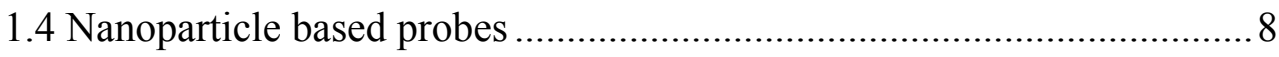

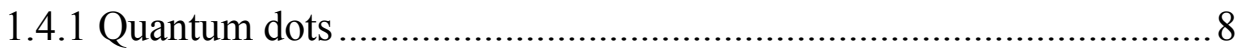

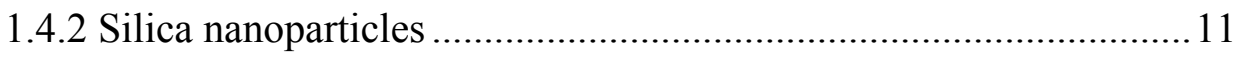

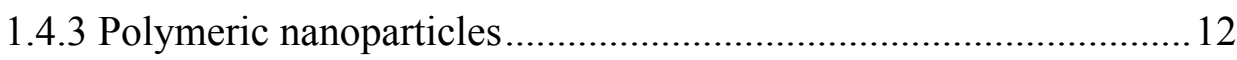


1.5 Keloid scar treatment by transdermal delivery .................................. 14

1.5.1 Conventional treatment for keloid scars ...................................... 15

1.5.2 Keloid fibroblasts engineering by transdermal delivery ................ 17

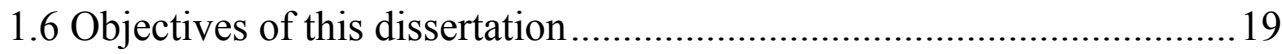

Chapter 2: Mesenchymal Stem Cell Imaging by Fluorescent and Bioresorbable Polymeric Nanoparticles with Enhanced Photostability................................ 22

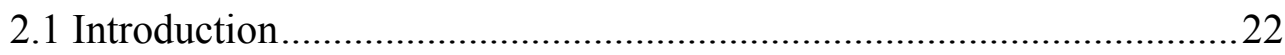

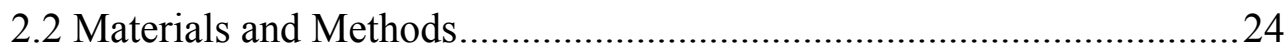

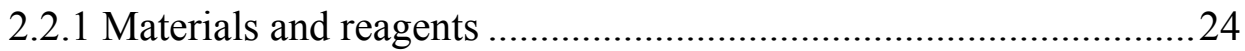

2.2.2 General materials characterization...........................................25

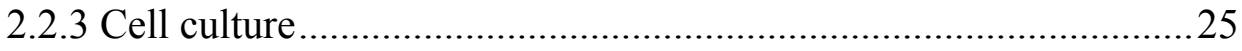

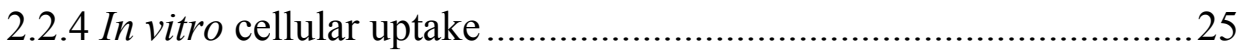

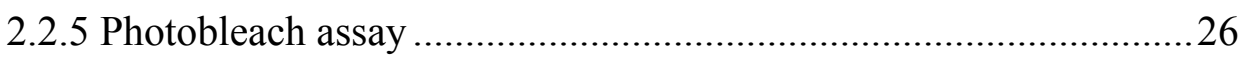

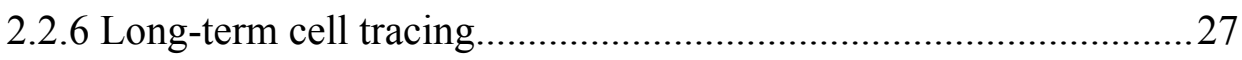

2.2.7 In vitro cytotoxicity.............................................................. 27

2.2.8 2,5-bis(6-hydroxyhexyl)-3,6-di(thiophen-2-yl)pyrrolo[3,4-c]pyrrole

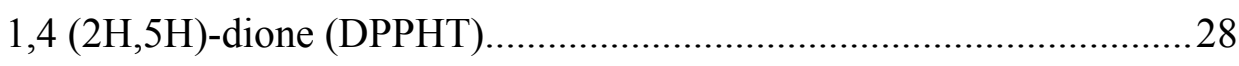

2.2.9 Synthesis of PCL-DPP-PCL and PCL-QA-PCL ........................29

2.2.10 General procedure for the synthesis of the PCL-DPP-PCL

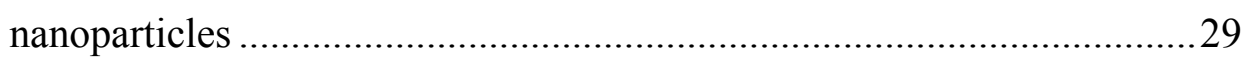

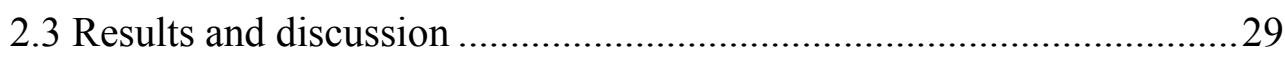

2.3.1 Fabrication and characterization of NPs...................................29

2.3.2 In vitro cellular uptake of NPs .................................................. 33

2.3.3 Photostability of NPs in cellular imaging ...................................35

2.3.4 Long-term cell tracing using PCL-DPP-PCL NPs vs. Qtracker® .. 43 


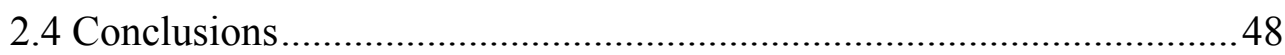

Chapter 3. Long-term Tracking Mesenchymal Stem Cell Differentiation with Photostable Fluorescent Nanoparticles ......................................................49

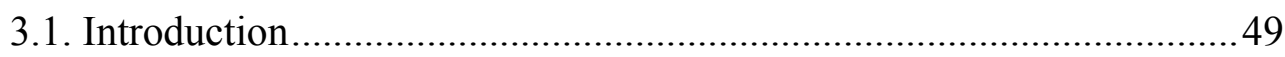

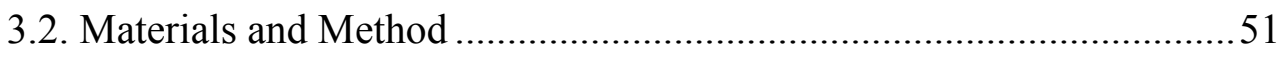

3.2.1 Preparation of PCL-DPP-PCL NPs ...........................................51

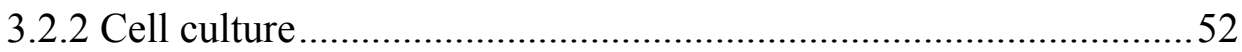

3.2.3 Cell labeling and flow cytometry.............................................52

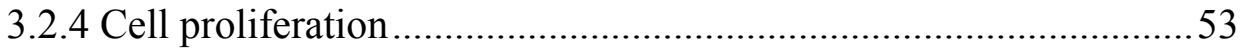

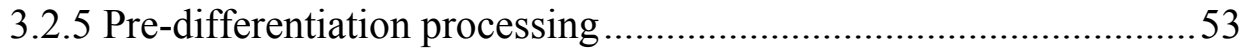

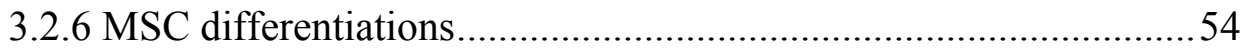

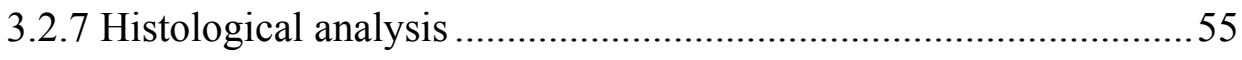

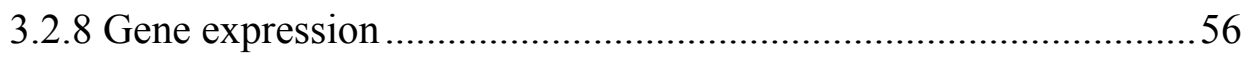

3.2.9 Confocal laser scanning microscopy ........................................57

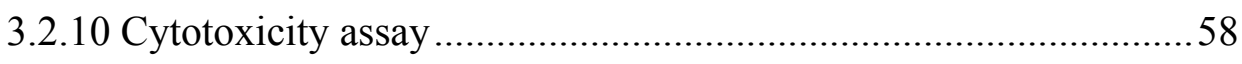

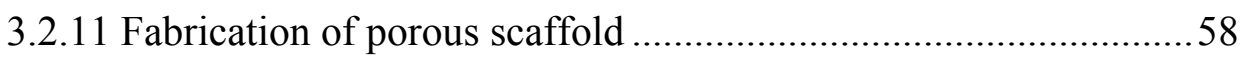

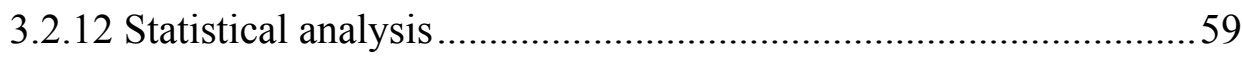

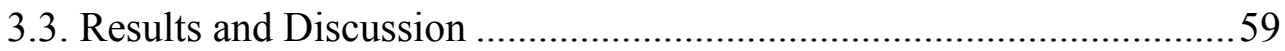

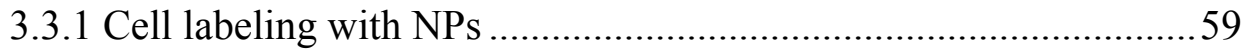

3.3.2 Multilineage differentiations of labeled hMSCs..........................63

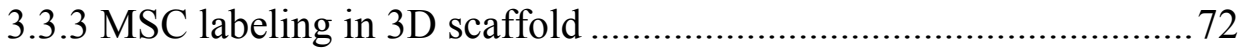

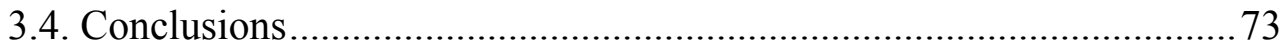

Chapter 4: Peptide Delivery with Poly (ethylene glycol) Diacrylate Microneedles for Keloid Scar Treatment........................................................ 75 


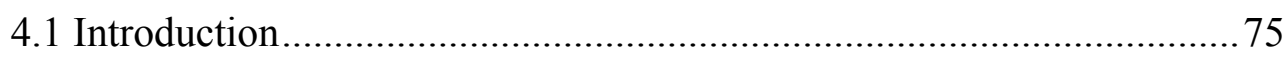

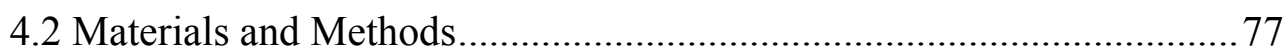

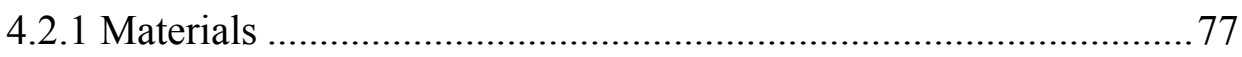

4.2.2 Fabrication of blank and CPT loaded PEGDA microneedles.........78

4.2.3 Swelling ratio and mesh size determination ................................78

4.2.4 Fabrication of hydrophilic molecules (FITC, FITC-Dextran, Gap 26)

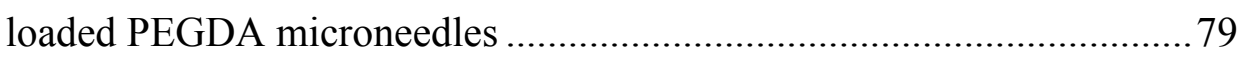

4.2.5 Release profile of CPT and Gap26 from microneedles ................ 80

4.2.6 Cell viability test by AlamarBlue ${ }^{\circledR}$ assay .................................... 80

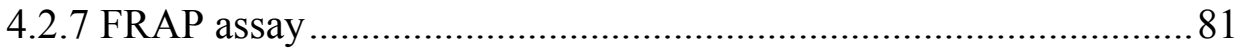

4.2.8 Real-time Polymerase Chain Reactions for collagen I expression .81

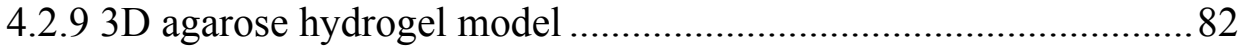

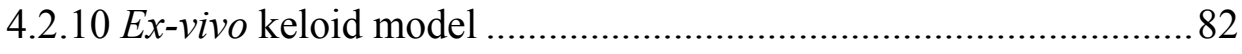

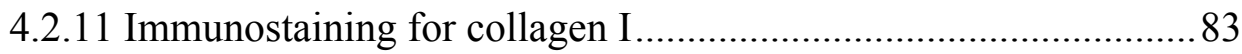

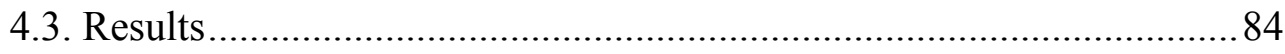

4.3.1 PEGDA microneedles loaded with hydrophobic camptothecin .....84

4.3.2 Swelling effect of PEGDA microneedles in aqueous solution .......86

4.3.3 Peptide loaded PEGDA microneedles ......................................... 88

4.3.4 Therapeutic effect of Gap26 loaded microneedles in ex-vivo keloid

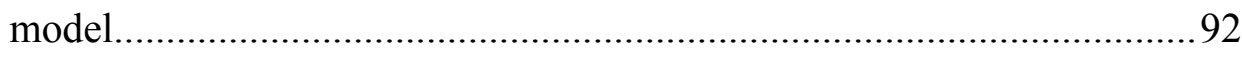

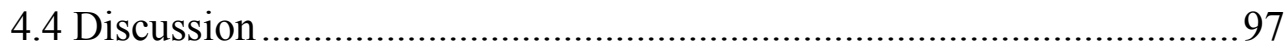

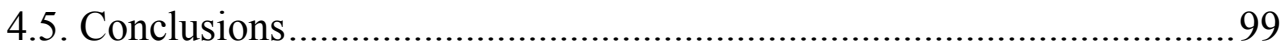

Chapter 5: Conclusions and Future Work..................................................... 100

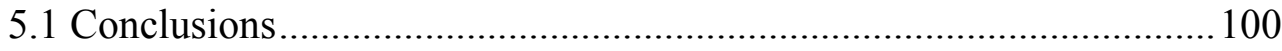

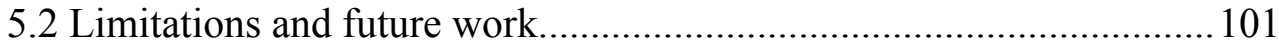


Reference . 103 


\section{SUMMARY}

Regenerative medicine is providing a promising treatment method for degenerative diseases, and has been extensively investigated in recent decades for bone, skin, cartilage, neurons, blood vessels, skin regeneration, and so on. One of the most important branches is to exploit multi-potent stem cells to differentiate into targeted tissues and organs before transplantation. Mesenchymal stem cells (MSCs) have been identified and widely explored as a promising and main source for regenerative medicine, with great differentiation potentials into osteocytes, adipocytes, and chondrocytes but less concern of ethical issues. However, a tough problem is to track the cell location, migration, fate and distribution of these transplanted cells, with clearer understanding about these will help instruct the optimum cell dosage, transplantation time, and evaluate efficacy. It is imperative to develop a photostable probe with excellent biocompatibility to track the MSCs for a long-term without affecting the cell proliferation and potency.

Another issue which is expected to be solved in this thesis is to develop an effective and conveniently used drug delivery system for treatment of keloid scars to achieve skin regeneration. Keloid scars, as a complex abnormal scar, cause not only aesthetic disfigure to the patients, but also have leaded to pruritus, infection, and much distress. Traditional treatments include surgery removal, silicone or pressure dressings, hypodermic injections of drugs like corticosteroid, fluorouracil (5-FU), and so on. However, these methods may result in pain, infections, and adverse effects as well as less compliance to patients. Transdermal delivery route by microneedles can provide a noninvasive method for delivering drugs with low cost and conveniences to patients.

This thesis targets to solve the above two problems by applying micro and nano technology. Specifically, enhancing the photostability of organic fluorophores will be achieved for long-term stem cell tracking in Chapter 2 and 3 by covalent 
linking organic dye di(thiophene-2-yl)-diketopyrrolopyrrole between a biocompatible polymer polycaprolactone and fabricating fluorescent nanoparticles based on this polymer. The cellular internalization of the prepared nanoparticles will be explored and cytotoxicity to the cells will be detected. In addition, photostability of these fluorescent nanoparticles will be compared to organic dyes, commercial quantum dots or physical blends of dye/polymer complexes. Furthermore, the long-term tracking of these photostable nanoparticles will be investigated in details for differentiations of mesenchymal stem cells into osteocytes, adipocytes, and chondrocytes and shown in Chapter 3.

In order to solve the second problem for keloid scar treatment, Chapter 4 will exhibit the application of biocompatible polymeric microneedles made from (polyethylene glycol) diacrylate (PEGDA) for loading of connexin mimetic peptide-Gap 26 by the swelling effect, which is expected to protect therapeutic biomolecules (including peptides) from enzymatic digestion that occurs in the oral route. Then the peptide-loaded microneedles will be applied to a keloid scar model and the effect of peptide on gap junction based intercellular communication and collagen I expression will be investigated in vitro and in an ex vivo model. 


\section{LIST OF FIGURES}

Figure 1. Differentiation potentials of adult human MSCs. (Adapted with permission from[22]. Vasileios Karantalis, Joshua M. Hare. Use of Mesenchymal Stem Cells for Therapy of Cardiac Disease. Circulation Research. 2015; 116 (8): 1413-1430. Copyright (2015) Wolters Kluwer Health, Inc. https://www.ncbi.nlm.nih.gov/pmc/articles/PMC4429294/).................... 6 Figure 2. (A) Rhodamine red and genetically encoded DsRed2 protein's absorption and emission. (B) Six QDs' absorption and emission spectra with the black line indicating the absorption of QDs emitted at $510 \mathrm{~nm}$. (C) Six QDs exhibit size-tunable fluorescence properties. Reprinted by permission from Macmillan Publishers Ltd: [Nature Materials][54], copyright (2005)............ 11

Figure 3. Photostability of QDs compared with Alexa 488 within single cell. (A) In the up row, QD-630-streptavidin (red) stained the nuclear antigens, while Alexa 488 (greeen) labeled microtubules in a 3 T3 cell. In the below row, QD630-streptavidin (red) stained microtubules, while Alexa 488 (green) labeled the nuclear antigens. During successive exposure to light from a usual mercury lamp $(100 \mathrm{~W})$ for $3 \mathrm{~min}$, the samples were taken images at indicated time points. Scale bar, $10 \mu \mathrm{m}$. (B) Normalized fluorescence intensity change curves of QD608-streptavidin and Alexa 488 which have stained microtubules and nuclear antigens respectively. Reprinted by permission from Macmillan Publishers Ltd: [Nature Biotechnology][53], copyright (2003).

Figure 4. Non-genetic cell engineering. (Reprinted from [82], copyright (2015) with permission from Elsevier)

Figure 5. Non-genetic cell engineering strategies including cell surface modification and delivering free drugs or encapsulated drugs by nanocarriers. (Reprinted from [82], copyright (2015) with permission from Elsevier) ........ 17

Figure 6. (a) Schematic showing the fabrication process of PCL-DPP-PCL nanoparticles by nanoprecipitation with Pluronic ${ }^{\circledR} 127$ as the stabilizer. (b-e) 
The morphology characterization and optical properties of PCL-DPP-PCL nanoparticles: (b) SEM image showing the average particle size of $51 \pm 4 \mathrm{~nm}$. (c) TEM image of PCL- DPP-PCL nanoparticles from a water dispersion. (d) The dynamic light scattering test shows that the hydrated NPs have an average diameter of $110 \mathrm{~nm}$ in water dispersion. (e) The fluorescence emission spectra of PCL-DPP-PCL nanoparticles (orange) and DPPHT in THF (blue) under 488 $\mathrm{nm}$ excitation. (This figure is reprinted with copyright from[111]) 31 Figure 7. (a) Absorption spectra of PCL-DPP-PCL NPs and DPPHT in THF and (b) linear plots for standard samples and PCL-DPP-PCL NPs. (This figure is reprinted with copyright from[111]) 32

Figure 8. Cellular uptake of PCL-DPP-PCL nanoparticles (NPs) in HuH-7 tumor cells (a-d and i) and MSCs (e-h and j) imaged by confocal laser scanning microscopy. The fluorescence of DAPI, Alexa Fluor ${ }^{\circledR} 633$ phalloidin, and NPs are represented with blue $(\mathbf{a}, \mathbf{e})$, red $(\mathbf{b}, \mathbf{f})$, and yellow $(\mathbf{c}, \mathbf{g})$, respectively. Merged images of HuH-7 cells and MSCs from three channels are shown in (d) and (h), respectively. (i) and (j) are ortho-view images of z-stack, showing nanoparticles inside the cells. Scale bars: $10 \mu \mathrm{m}(\mathrm{HuH}-7)$ and $20 \mu \mathrm{m}$ (MSC). For a, b, c, d, and i, pixel dwell time: $0.39 \mu$ s; frame size: $1024 \times 1024$; pixel size: $0.086 \mu \mathrm{m}$. For e, f, g, h, and j, pixel dwell time: $1.00 \mu \mathrm{s}$; frame size: $512 \times 512$; pixel size: $0.45 \mu \mathrm{m}$. (This figure is reprinted with copyright from[111])........34 Figure 9. Photostability of fluorescent PCL-DPP-PCL nanoparticles (NPs) compared to Alexa Fluor ${ }^{\circledR} 633$ phalloidin and DAPI in MSC. The fluorescences from DAPI, nanoparticles, and Alexa Fluor ${ }^{\circledR} 633$ phalloidin are represented by blue, yellow and red, respectively. (a) Chronological decay of fluorescence intensity of a multiply-stained MSC at different time points imaged by confocal laser scanning microscopy. The whole image field was photobleached. Pixel dwell time: $0.50 \mu \mathrm{s}$; frame size: $1024 \times 1024$; pixel size: $0.28 \mu \mathrm{m}$. Scale bar: 20 $\mu \mathrm{m}$. (b) Relative intensity (instantaneous intensity / initial intensity) change of three fluorophores in the photobleached region. Curves are fitted using mono- 
exponential (PCL-DPP-PCL NPs and DAPI) or bi-exponential functions (Alexa Fluor ${ }^{\circledR} 633$ phalloidin). The nominal powers of 405, 561, and $633 \mathrm{~nm}$ lasers are $20 \mathrm{~mW}, 20 \mathrm{~mW}$, and $5 \mathrm{~mW}$, respectively. (This figure is reprinted with

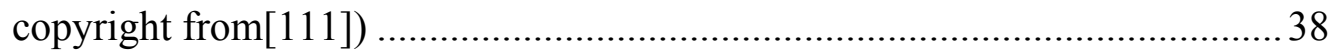

Figure 10. SEM (a, d), TEM (b, e) and DLS (c, f) characterization of physically blended DPPHT/PCL NPs (a-c) and PCL-QA-PCL NPs (d-f). (g) The chemical structure of PCL-QA-PCL. (This figure is reprinted with copyright from[111]) 40

Figure 11. Cellular uptake of physically blended PCL/DPPHT NPs (a-d and i) and PCL-QA-PCL nanoparticles (e-h and j) in MSCs imaged by confocal laser scanning microscopy. The fluorescence of DAPI, Alexa Fluor ${ }^{\circledR} 633$ phalloidin, physically blended PCL/DPPHT NPs, and PCL-QA-PCL nanoparticles are pseudo-labeled with blue (a, e), red (b, f), yellow (c), and green (g), respectively. Merged images of MSCs from different channels are shown in (d) and (h), respectively. (i) and (j) are ortho-view images of z-stack, showing they could be uptaken by the cells. Scale bars: $20 \mu \mathrm{m}$. (For a-d and i, pixel dwell: $1.27 \mu \mathrm{s}$, frame size: $512 \times 512$; for $\mathrm{e}-\mathrm{h}$, and $\mathrm{j}$, pixel dwell: $0.63 \mu \mathrm{s}$, frame size: $1040 \times 1040$ ). (This figure is reprinted with copyright from[111]). 40

Figure 12. Photostability of physically blended PCL/DPPHT NPs compared to Alexa Fluor ${ }^{\circledR} 633$ phalloidin and DAPI in a MSC. The fluorescence of DAPI, physically blended PCL/DPPHT NPs, and Alexa Fluor ${ }^{\circledR} 633$ phalloidin are represented with blue, yellow and red, respectively. (a) Chronological decay of fluorescence intensity of a multiply-stained MSC at different time points imaged by confocal laser scanning microscopy. The green polygon indicates the region photobleached. Pixel dwell time: $1.27 \mu$ s; frame size: $512 \times 542$; pixel size: $0.37 \mu \mathrm{m}$. Scale bar: $20 \mu \mathrm{m}$. (b) Relative intensity (instantaneous intensity / initial intensity) change of three fluorophores in the photobleached region. Curves are fitted using mono-exponential (DAPI) or bi-exponential functions 
(physically blended PCL/DPPHT nanoparticles and Alexa Fluor® 633 phalloidin). (This figure is reprinted with copyright from[111])...................41

Figure 13. Photostability of PCL-QA-PCL NPs compared to Alexa Fluor ${ }^{\circledR} 633$ phalloidin and DAPI in a MSC. The fluorescences from DAPI, PCL-QA-PCL nanoparticles, and Alexa Fluor ${ }^{\circledR} 633$ phalloidin are represented with blue, green, and red, respectively. (a) Chronological decay of fluorescence intensity of a multiply-stained MSC at different time points imaged by confocal laser scanning microscopy. The whole image field was photobleached. Pixel dwell time: $0.64 \mu \mathrm{s}$; frame size: $1024 \times 1024$; pixel size: $0.42 \mu \mathrm{m}$. Scale bar: $20 \mu \mathrm{m}$. (b) Relative intensity (instantaneous intensity / initial intensity) change of three fluorophores in the photobleached region. Curves are fitted using monoexponential (DAPI) or bi-exponential functions (PCL-QA-PCL NPs and Alexa Fluor $^{\circledR} 633$ phalloidin). (This figure is reprinted with copyright from[111]).. 42 Figure 14. Long-term tracing of HuH-7 tumor cells using (a, c) PCL-DPP-PCL nanoparticles and (b, d) Qtracker ${ }^{\circledR}$. The fluorescence intensity change of the labeled cells was measured by flow cytometry analyses at different time points. The control represents the unlabeled cells. The percentages of cells with positive signals in (c, d) were identified based on the gating thresholds shown in (a, b) for NP and Qtracker ${ }^{\circledR}$, respectively. (This figure is reprinted with copyright from[111]) 45

Figure 15. Long-term tracing of HuH-7 tumor cells using PCL-DPP-PCL NPs and Qtracker ${ }^{\circledR}$. The fluorescence intensity change of the labeled cells is characterized by optical contrast and fluorescence microscopy measured at different time points. (This figure is reprinted with copyright from[111])..... 46 Figure 16. Long-term tracing of MSCs using PCL-DPP-PCL NPs and Qtracker ${ }^{\circledR}$. The fluorescence intensity change of the labeled cells is characterized by (a) flow cytometry histograms with PCL-DPP-PCL NPs, (b) flow cytometry histograms with Qtracker, (c) flow cytometry dot plots with PCL-DPP-PCL NPs, (d) flow cytometry dot plots with Qtracker, (e) optical 
contrast and fluorescence microscopy measured at different time points. (This figure is reprinted with copyright from[111])

Figure 17. Cell viabilities of HuH-7 and MSCs after incubating with different concentrations of PCL-DPP-PCL NPs for (a) $24 \mathrm{~h}$ and (b) $72 \mathrm{~h}$ tested by PrestoBlue assay. Blank cells without any nanoparticles treatment were regarded as control. Values were expressed as means $\pm S D(n=3)$. Paired student t-test was used for statistical analysis between groups treated with different concentrations of NPs and control $(p>0.5)$. (This figure is reprinted with copyright from[111])

Figure 18. Schematic illustration of long-term tracking human mesenchymal stem cells during adipogenic, chondrogenic and osteogenic differentiations using PCL-DPP-PCL nanoparticles. (This figure is reprinted with permission from[144]. Copyright (2016) American Chemical Society) .60

Figure 19. Corresponding ortho-views by taking different $\mathrm{z}$ stacks for the confocal laser scanning microscope images of NP-ST (A) and NP-LT (B) in Figure 21A. Cell nuclei were counterstained with DAPI (blue), F-actin were stained with Alexa Fluor ${ }^{\circledR} 633$ phalloidin (red), and nanoparticles were represented as yellow. (This figure is reprinted with permission from[144]. Copyright (2016) American Chemical Society) 61

Figure 20. Relative cell viability of hMSCs during NP-labelling (concentration: $0.2 \mathrm{mg} / \mathrm{mL}$ ) detected with PrestoBlue assay. The viability of blank cells without labelling was set as $100 \%$. The data were expressed as means \pm SD $(n=3)$. (This figure is reprinted with permission from[144]. Copyright (2016) American Chemical Society) 61

Figure 21. (A) Cellular uptake of NPs in hMSCs imaged by confocal microscopy. Cell nuclei were counterstained with DAPI (blue), F-actin were stained by Alexa Fluor ${ }^{\circledR} 633$ phalloidin (red) and the nanoparticles were represented as yellow. Scale bar: $100 \mu \mathrm{m}$. (B) Quantitative analysis of cellular uptake of NPs by flow cytometry. Normalized MFI: 1143.5 for NP-LT, 691.1 
for NP-ST. (C) Cell proliferation measured by PrestoBlue assays. Data presented as mean $\pm \mathrm{SD}$. ${ }^{*} \mathrm{p}$-value $<0.05, * * \mathrm{p}$-value $<0.01 . \mathrm{n}=3$. (This figure is reprinted with permission from[144]. Copyright (2016) American Chemical Society) 63

Figure 22. Microscopic images of the NP-labeled hMSCs during adipogenic differentiation. The images of NP-ST and NP-LT groups were obtained by merging the bright field contrast images and the corresponding fluorescent images. Scale bar: $250 \mu \mathrm{m}$. (This figure is reprinted with permission from[144]. Copyright (2016) American Chemical Society) 65

Figure 23. MSC differentiation in adipogenic lineage. (A) Confocal fluorescence images of the differentiated adipocytes (day 21). Scale bar: 100 $\mu \mathrm{m}$. (B) The retained fluorescence due to NP staining in the differentiated adipocytes (day 21) analyzed by flow cytometry. Normalized MFI: 394.7 for NP-LT, 208.8 for NP-ST. (C) The mRNA expression of leptin and PPAR- $\gamma$ (day 14) quantified by RT-PCR assay. The results were normalized to the data on day 0 (before differentiation). ${ }^{*}$ p-value $<0.05,{ }^{*}$ p-value $<0.01$. Data presented as mean \pm SD. $n=4$ for leptin and $n=3$ for PPAR- $\gamma$. (D) Oil Red staining for oil droplets in the differentiated adipocytes (day 21). Scale bar: $250 \mu \mathrm{m}$. (This figure is reprinted with permission from[144]. Copyright (2016) American Chemical Society) 66

Figure 24. Analysis of the total positive area of Oil Red O (ORO) staining against the reference area of the image frame according to a threshold range set with ImageJ. There is no significant difference among three groups (p-value $>$ $0.05, \mathrm{n}=7$ ). The area is measured based on an arbitrary unit (a.u.). (This figure is reprinted with permission from[144]. Copyright (2016) American Chemical Society) 67

Figure 25. MSC differentiation in chondrogenic lineage. (A) The cryosections of the hMSC-derived tissue pellets (day 28). Scale bar: $100 \mu \mathrm{m}$. (B) Confocal fluorescence images of the original pellets (day 28). Scale bar: $200 \mu \mathrm{m}$. (C) 
Flow cytometry analysis of the trypsinized chondrocytes (day 28). Normalized MFI: 80.4 for NP-ST. (D-F) The mRNA expression of Col1, Col2 and aggrecan (day 21) quantified by RT-PCR assay. Data were normalized according to the results at day 0 (before differentiation). Data presented as mean \pm SD. $n=5$ for Col1, Col2 and Col2/Col1, $n=3$ for AGG. (G) Immunohistochemistry staining of Col2 in the chondrogenic pellets (day 28). Scale bar: $250 \mu \mathrm{m}$. (This figure is reprinted with permission from[144]. Copyright (2016) American Chemical Society) .69

Figure 26. MSC differentiation in osteogenic lineage. (A) Confocal fluorescence images of the differentiated osteocytes (day 21). Scale bar: 100 $\mu \mathrm{m}$. (B) Alizarin Red histological staining for calcium deposition after osteogenic differentiation (day 21). Scale bar: $250 \mu \mathrm{m}$. (C) The mRNA expression of Col1, OCN and ALP (day 14) quantified by RT-PCR assay. Data were normalized according to the results at day 0 (before differentiation). *pvalue $<0.05, * *$ p-value $<0.01$. Data presented as mean \pm SD. $n=4$. (D) Flow cytometry analysis of the hMSC-derived osteocytes (day 21). Normalized MFI: 94.6 for NP-ST. (This figure is reprinted with permission from[144]. Copyright (2016) American Chemical Society) 71

Figure 27. Scanning electron microscope images for PLCL porous scaffold under different magnifications: (A) $100 \times$, (B) $250 \times$ and (C) $500 \times$.(This figure is reprinted with permission from[144]. Copyright (2016) American Chemical Society) .73

Figure 28. Confocal fluorescence images of hMSCs seeded in a 3D scaffold (day 14). 3D images were reconstructed using ZEN 2012 software by taking different z-stack images. Scale bar: $100 \mu \mathrm{m}$. (This figure is reprinted with permission from[144]. Copyright (2016) American Chemical Society) .........73

Figure 29. Schematic showing the loading of hydrophilic peptides into poly(ethylene glycol) diacrylate microneedles by the swelling effect. (This figure is reprinted with copyright from[170]) 77 
Figure 30. The inhibition of (A) the proliferation and (B) collagen I expression of keloid fibroblasts by camptothecin (CPT). ** $\mathrm{p}<0.01, * * * \mathrm{p}<0.001$. (This figure is reprinted with copyright from[170]) ........................................... 84

Figure 31. (A) Stereomicroscope images of blank PEGDA microneedles, scale bar: $500 \mu \mathrm{m}$. (B) Scanning electron microscopy (SEM) images of blank PEGDA microneedles, scale bar: $100 \mu \mathrm{m}$. (This figure is reprinted with copyright from[170]) .85

Figure 32. (A) Confocal images of CPT loaded microneedles. Scale bar: 100 $\mu \mathrm{m}$. (B) Release profile of CPT from the microneedle patch in PBS. (C) Confocal images of agarose hydrogel containing keloid fibroblasts after the treatment with PEGDA microneedle patches for $24 \mathrm{~h}$, scale bar: $100 \mu \mathrm{m}$. (D) Anti-proliferative effect of CPT loaded microneedles against keloid fibroblasts encapsulated in agarose gel; $* \mathrm{p}<0.05$. (This figure is reprinted with copyright from[170]). .87

Figure 33. Swell performance of PEGDA microneedles and loading molecules with different molecular weight. (A) Swelling ratio of PEGDA microneedles prepared with different UV exposure time. ${ }^{* *} p<0.01$. (B) Confocal images of PEGDA microneedles loaded with FITC and FITC-Dextran with different molecular weight. Scale bar: $100 \mu \mathrm{m}$. (C) Quantified microneedle fluorescence intensity in B relative to that with free FITC. (This figure is reprinted with copyright from[170]) .87

Figure 34. Mesh size of PEGDA microneedles prepared with different UV exposure time. ** $\mathrm{p}<0.01$. (This figure is reprinted with copyright from[170]) .88

Figure 35. Effect of Gap 26 on FRAP of keloid fibroblasts and loading of FITCGap 26 into PEGDA microneedles. (A) Confocal images of keloid fibroblasts in FRAP experiment with the treatment of Gap 26. Scale bar: $20 \mu \mathrm{m}$. (B) Recovered fluorescence intensity change during fluorescence recovery. 
$* \mathrm{p}<0.05, \quad * * \mathrm{p}<0.01$. (C) Confocal images of Gap26 loaded PEGDA microneedles. Scale bar: $100 \mu \mathrm{m}$. (D) Quantitative analysis of C. (This figure is reprinted with copyright from[170]) 91

Figure 36. Cumulative release of Gap26 from the microneedle patch. (This figure is reprinted with copyright from[170]) 92

Figure 37. Ex-vivo keloid scar model and injection of keloid fibroblasts. (A) Illustration of the ex-vivo keloid model. (B) Presence of DiI labelled keloid fibroblasts in the dermis region of skin samples. Scale bar: $100 \mu \mathrm{m}$. (This figure is reprinted with copyright from[170]) 93

Figure 38. Keloid fibroblasts labelled with DiI. scale bar: $100 \mu \mathrm{m}$. (This figure is reprinted with copyright from[170]) 93

Figure 39. Effect of Gap 26 loaded microneedles on collagen I expression of skin samples in the ex vivo model. (A) Immunostaining of Collagen I expression after the treatment with Gap26-loaded microneedles. Scale bar: $100 \mu \mathrm{m}$. (B) Quantitative analysis of collagen I expressions in A by normalization to the modified skin without treatment. (This figure is reprinted with copyright from[170]) 95

Figure 40. (A) Immunostaining of Collagen I expression in the ex vivo keloid model after the treatment with CPT loaded microneedles. Scale bar: $100 \mu \mathrm{m}$. (B) Quantitative analysis of collagen I expressions in A by normalization to the keloid model without treatment. (This figure is reprinted with copyright from[170]) 96

Figure 41. Stereomicroscope images of CPT and Gap26 loaded PEGDA microneedles after application in the ex vivo skin samples, scale bar: $500 \mu \mathrm{m}$. (This figure is reprinted with copyright from[170]) 96 


\section{LIST OF TABLES}

Table 1. Parameters for fitting relative intensity change of PCL-DPP-PCL NPs in MSC (Figure 9b) using $\mathrm{y}=\mathrm{A}_{1} * \exp \left(-\mathrm{t} / \tau_{1}\right)+\mathrm{y}_{0}$ (Note: In this bi-exponential model, the half-life $(\mathrm{t} 1 / 2 *)$ was calculated by $\ln 2 * \tau 2$.) (This table is reprinted

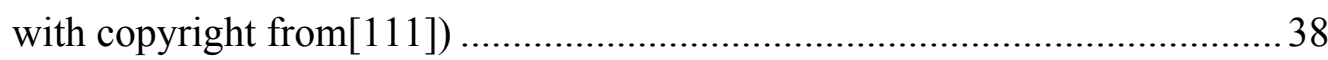

Table 2. Parameters for fitting relative intensity change of DAPI in MSC (Figure 9b) using $y=A_{1} * \exp \left(-t / \tau_{1}\right)+y_{0}$ (Note: In this bi-exponential model, the half-life $(\mathrm{t} 1 / 2 *)$ was calculated by $\ln 2 * \tau 2$.) (This table is reprinted with copyright from[111]) 39

Table 3. Parameters for fitting relative intensity change of Alex Fluor ${ }^{\circledR} 633$ in MSC (Figure 9b) using $\mathrm{y}=\mathrm{A}_{1} * \exp \left(-\mathrm{t} / \tau_{1}\right)+\mathrm{A}_{2} * \exp \left(-\mathrm{t} / \tau_{2}\right)+\mathrm{y}_{0}$ (Note: In this biexponential model, the half-life $(\mathrm{t} 1 / 2 *)$ was calculated by $\ln 2 * \tau 2$.) (This table is reprinted with copyright from[111]) 39

Table 4. Primer sequences for specific genes used in RT-PCR. (This table is reprinted with permission from[144]. Copyright (2016) American Chemical Society) .57

Table 5. Primer sequences for real-time polymerase chain reactions (This table is reprinted with copyright from[170]) .82

Table 6. FITC-Gap 26 loading amount and efficiency into PEGDA microneedles by swelling effect. (This table is reprinted with copyright from[170]) 91 


\section{LIST OF ABBREVIATIONS}

\begin{tabular}{|c|c|}
\hline Abbreviations & Full Name \\
\hline MSCs & Mesenchymal stem cells \\
\hline ESCs & Embryonic stem cells \\
\hline FDA & Food and drug administration \\
\hline UCB & Umbilical cord blood \\
\hline iPSCs & Induced pluripotent stem cells \\
\hline MRI & Magnetic resonance imaging \\
\hline NPs & Nanoparticles \\
\hline GFP & Green fluorescent protein \\
\hline QDs & Quantum dots \\
\hline $\mathbf{C x}$ & Connexin \\
\hline DMEM & Dulbecco's modified eagle medium \\
\hline FBS & Fetal bovine serum \\
\hline PBS & Phosphate buffer saline \\
\hline EDTA & Ethylenediaminetetraacetic acid \\
\hline DPP & Di(thiophene-2-yl)-diketopyrrolopyrrole \\
\hline PCL & Polycaprolactone \\
\hline PPAR- $\gamma$ & Peroxisome proliferator-activated receptors \\
\hline Col I & Collagen type 1 \\
\hline
\end{tabular}




\begin{tabular}{|c|c|}
\hline Col II & Collagen type 2 \\
\hline Agg & Aggrecan \\
\hline OCN & Osteocalcin \\
\hline ALP & Alkaline phosphatase \\
\hline RT-PCR & Messenger ribonucleic acid \\
\hline mRNA & Complementary DNA \\
\hline cDNA & Cycle threshold \\
\hline GAPDH & Glyceraldehyde 3-phosphate dehydrogenase \\
\hline CT & Microneedle \\
\hline DAPI & Polyethylene glycol) diacrylate \\
\hline MN & Fluorescence recovery after bleach \\
\hline PEGDA & Polydimethylsiloxane \\
\hline FRAP & Ultraviolet \\
\hline PDMS & Analysis of variance \\
\hline UV & \\
\hline ANOVA & \\
\hline & \\
\hline & \\
\hline & \\
\hline & \\
\hline & \\
\hline & \\
\hline
\end{tabular}




\section{Chapter 1: Introduction}

\subsection{Regenerative medicine}

Human tissue and organs may be damaged by age, disease, or trauma. The limited source for transplantations and consequent immune problems require the development of another kind of novel therapy, which is able to regenerate tissues and decrease the reliance on transplantations. Regenerative medicine emerged as a promising field which targets to promote tissue regeneration by exploiting the multi-disciplinary expertise, including engineering and life science principles, which is potentially able to restore diseased and injured tissues and even the whole organs. Specifically, regenerative medicine aims to repair cells, tissues, and organs by exploiting the therapeutic value of body's own growth factors and stem cells[1-4]. It can utilize autologous or allogeneic stem cells to enhance the intrinsic healing processes and supplement repair deficiencies. By replacing, engineering, or regenerating human cells, tissues or organs can be regenerated to restore or establish normal function.

Since the appearance of the regenerative field several decades ago, it has received increasing attention and achieved much progress. A number of therapies especially for wound healing and orthopaedics applications, have obtained the approval from Food and Drug Administration (FDA) and are currently commercially available[1].

Among the strategies in regenerative medicine, many of them deliver autologous or allogeneic therapeutic and typically differentiated cells, which still have proliferation capacity[1]. For example, Carticel utilizes autologous chondrocytes, which are collected from articular cartilage, expanded ex vivo, and then implanted into the site of injury, to treat focal articular cartilage defects and has been approved by FDA in the orthopaedic field. This product leads to effective recovery and the result is comparable with that observed by microfracture and mosaicplasty techniques. There are also other examples like 
laViv, targeting to improve the nasolabial fold wrinkles by injection of the autologous fibroblasts, and Epicel for severe burn wounds by autologous keratinocytes. These autologous cells are collected from a patient's tissue, and often require a long time before treatment for the cell expansion in vitro. Another promising candidate is the allogeneic cell source with low antigenicity, which can allow off-the-shelf tissues with low risk of immune reaction. Other FDA-approved examples include GINTUIT and Apligraf, which exploit allogeneic human foreskin fibroblasts during the fabrication of wound-healing grafts.

\subsection{Stem cell platform}

With the direct contribution to innate healing, stem cells have been widely recognized to provide a promising source for regenerative medicine. In fact, there are many types of stem cells either extracted or bioengineered by molecular programming to provide enormous progenitor cells, which are endowed with multi-potency and can be applied for various regenerative applications. Stem cells from natural sources include embryonic stem cells, perinatal stem cells (such as stem cells derived from umbilical cord), as well as adult stem cells (such as stem cells derived from bone marrow including mesenchymal stem cells (MSCs)). In addition, iPSCs technology pioneered by Shinya Yamanaka's lab in Kyoto, Japan, also attracts much attention which can program adult cells and induce them to become pluripotent stem cells by gene encoding.

\subsubsection{Embryonic Stem Cells}

Embryonic stem cells (ESCs), derived from the inner cell mass of a preimplantation blastocyst, have the pluripotent capacity and can differentiate into various cell types, including neuron-like cells, dendritic cells, pancreatic islet-like cells, osteocytes, chondrocytes, adipocytes, skin, and so on. This amazing potency makes ESCs be able to produce a large number of transplantable cells for therapeutic use. In order to characterize pluripotency of 
human ESC, several marker expressions can be detected, such as alkaline phosphatase, POU transcription factor Oct3/4, Nanog, Cripto/TDGF1, proteoglycans TRA-1-60/81, GCTM-2, and embryonic antigens SSEA-3 and SSEA-4. Early lineage commitment of the three germinal layers has been monitored by the expression of markers such as Sox 1 for neuroectoderm, Pdx1 for endoderm, and Flk-1 for mesoderm.

Currently, there are many clinical trials using ESCs-derived cells, including retinal pigment epithelium[5], pancreatic endoderm derivatives (for type 1 diabetes)[6], and cardiac progenitors (for severe heart failure)[7]. However, there is an unavoidable concern for the regenerative application of ESCs, that is the risk of teratoma formation after transplantation due to their unlimited growth capacity. However, further clarifications about embryonic developmental pathways to induce ESC into tissue-specific lineages can potentially solve this problem. On the other hand, proper regulation of ESC differentiation before and after transplantation is also very important for the safety in the clinical application.

\subsubsection{Perinatal Stem Cells}

In addition to ESCs, an increasing evidence has proved the differentiation capacity of perinatal stem cells, such as the ones from umbilical cord blood (UCB). These cells also exhibit stemness properties and are normally collected at the birth. UCB-derived stem cells transplantation with less immune tolerance, can lead to high degree of engraftment and less serious graft-versus-host disease[2]. And these UCB-derived stem cells can also be expanded, continuously cultured in vitro, and also differentiate into three embryonic germinal layers, including the endoderm (like mature hepatocytes, etc), mesoderm (like adipocytes, chondrocytes, osteoblasts, etc), and ectoderm (like neurons, etc)[2]. UCB transplantations have been successfully performed in more than 10,000 patients which have genetic, hematological or immunological disorders[8]. 
A main limitation of UCB transplantation is the low cell dose of UCB derived stem cells, thus resulting in an increasing risk of graft failure[9]. Besides, it's necessary to have a bigger investment in the public cord blood tanks considering the importance of HLA-matching and cell dose on outcomes after UCB transplantation. And there should be accredited cord blood banks and bone marrow donor registries especially for patients who don't have an HLAmatched sibling to benefit hematopoietic stem cell transplantation[8].

\subsubsection{Engineered stem cells}

The advanced development of biotechnology allows to perform genetic reprogramming for adult cells and render them with the embryonic differentiation abilities. These novel technologies including nuclear reprogramming can obtain the pluripotent stem cells without the need of embryo extraction. In 2006, Shinya Yamanaka's group in Japan achieved a great breakthrough in which they reprogrammed mouse skin fibroblasts into stem cells by overexpressing the stemness regulators[10], this marked the appearance of induced pluripotent stem cells (iPSCs). Thereafter, they and other groups in the world, such as James Thomson and George Daley also successfully converted human fibroblasts or somatic cells into human iPSCs[11-13]. Ever since, iPSCs attract increasing attention because they can avoid the ethical issues from using human embryos and the genetic programming can match to the donor[14-16].

Up to now, the clinical applications about iPSCs have not achieved a lot, with one which used autologous patient-specific iPSC derivatives for the treatment of age-related macular degeneration[17]. Starting from September 2014 to treat the first patient in Japan[18], the clinical trial was recently paused since the genetic mutations were found in the iPSCs from a second patient.

\subsubsection{Mesenchymal stem cells}

Adult stem cells are able to provide an abundant sources for progenitors from bone marrow, adipose tissue, as well as resident stem cell pools, compared with 
the above stem cells[19]. Considering the easy accessibility, autologous status, and proliferative potential, these adult stem cells become the main source in the regenerative medicine clinically[20].

Although these stem cells may also be extracted from adipose tissue or the resident stem cell pool, currently the main source is from bone marrow. Mesenchymal stem cells (MSCs) with different potentials can be produced from bone marrow with high efficiency from the supporting architecture of the adult marrow, attracting an increasing attention for the regenerative medicine applications.

Mesenchymal stem cells can demonstrate multipotency to regenerate the bone, cartilage, muscle, and so on (Figure 1)[21]. They are also discovered to regenerate liver and pancreatic islet cell, as well as play a role in the protection of kidney, heart, or lung injury. In addition, by secreting various bioactive molecules, the regenerative microenvironment generated by mesenchymal stem cells can restrict the damage area and initiate a self-adjusted regenerative response.

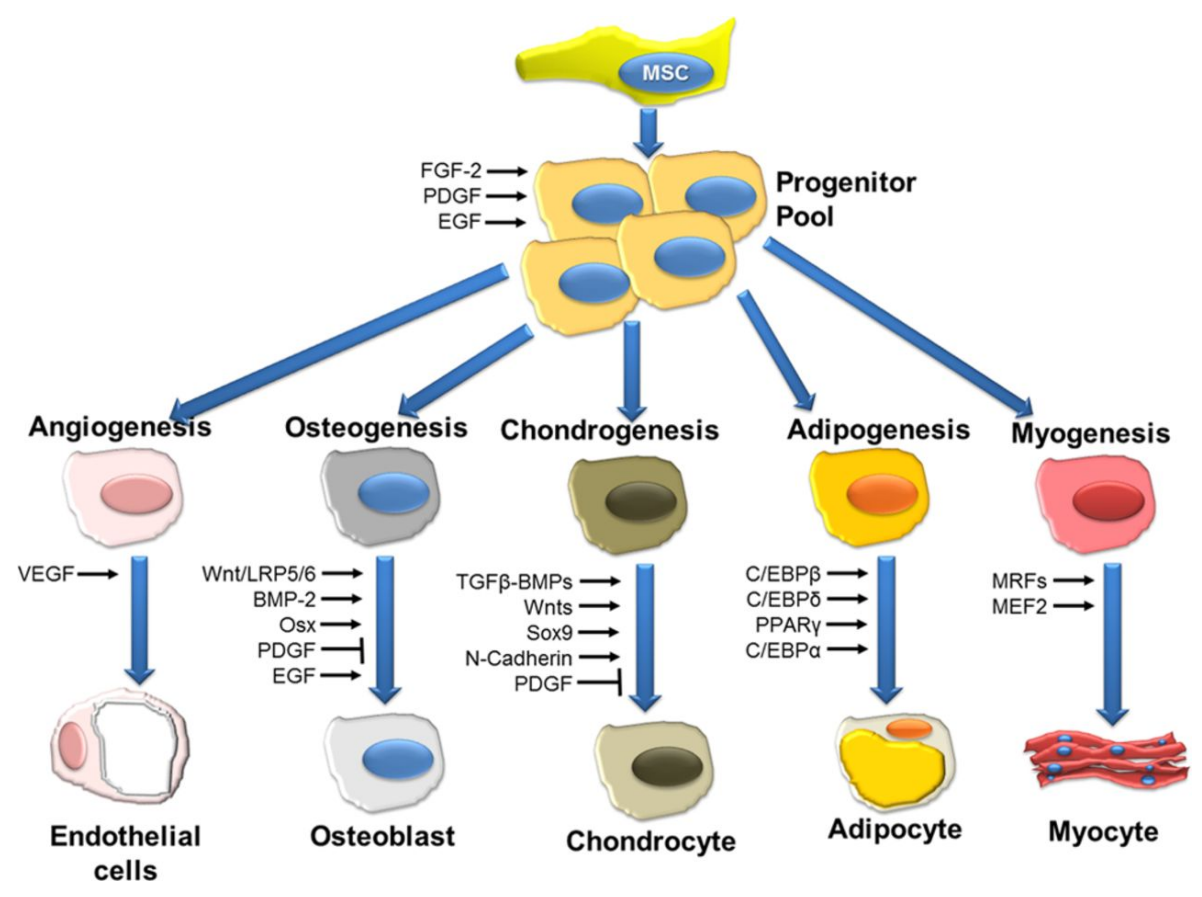


Figure 1. Differentiation potentials of adult human MSCs. (Adapted with permission from[22]. Vasileios Karantalis, Joshua M. Hare. Use of Mesenchymal Stem Cells for Therapy of Cardiac Disease. Circulation Research. 2015; 116 (8): 1413-1430. Copyright (2015) Wolters Kluwer Health, Inc. https://www.ncbi.nlm.nih.gov/pmc/articles/PMC4429294/)

Current clinical trials include mesenchymal stem cells from both endogenous and exogenous tissues, in the applications for treatment of osteogenesis imperfecta, Crohn's disease, and graft-versus-host disease[23]. It's favorable to apply the allogeneic mesenchymal stem cell to treat some acute diseases, such as acute myocardial infarction, and so on, since preparing allogeneic cells can efficiently save time without need to extract and expand autologous cells from the recipient. However, if the mesenchymal stem cells are not needed urgently for some patients, it will be better to extract and expand patients' cells before implantation as personalized cell-based therapy[24].

\subsection{Mesenchymal stem cell tracking}

The past decades also witnessed the increasing researches and achievements in stem cell field, which have broadened the treatment for many diseases, such as diabetes, neurodegenerative or cardiovascular diseases. Human stem cells, especially mesenchymal stem cells (MSCs), owning the self-renew ability and high differentiation potency, can provide an abundant source for the wide application in regenerative medicine, which is very promising to revolutionize the treatment of various diseases[22, 25, 26]. However, although many results in vitro or in vivo are promising, there are still many issues which are needed to be solved before human use. One of the problems is the shortage of methods to understand the fate, distribution, and the function of transplanted stem cells in the local microenvironment to make sure these transplanted cells have arrived in the sites of injury[27]. Previously, monitoring the fate of transplanted stem cells has to depend on the histological studies at the desired time points after the transplantation. Therefore, it's very necessary to develop a non-invasive imaging methods to real-time monitor the transplanted stem cells, which can benefit the optimization conditions for cell dosage, delivery route, and time 
points of transplantations, as well as prediction of the therapy efficacy[28, 29]. The long-term tracking technique will help researchers have a better understanding about the distribution, motility, location, and roles of stem cells in the transplanted scaffold or tissue engineered organs, and further analyze their interaction with the patient's cells and body[22, 25, 26, 30, 31].

In order to achieve stem cells tracking, magnetic resonance imaging (MRI) is commonly used[32-34]. Many researchers developed magnetic contrast agents to try to achieve MRI in vivo by superparamagnetic iron oxide nanoparticles (SPIONs). For example, by coating the dextran or other polymers on the nanoparticles' surface, their stability and solubility can be increased and this way also reduces aggregation, which provides a useful tracking method for stem cells in MRI and is much better than the traditional gadolinium-based contrast agents. However, using this dextran coated SPIONs also causes problems. It is reported that stem cells are unfavorable to internalize these SPIONs by endocytosis; therefore, high labeling efficiency has to be achieved by using higher concentrations of the nanoparticles (NPs) and additional transfection agents. On the other hand, iron oxide NPs have been reported to promote the free hydroxyl radicals and reactive oxygen molecules after dissolving within cells, which may lead to some toxic effects to induce apoptosis of cell and alter the cell metabolism. What's more, $\mathrm{Fe}^{2+}$ irons dissolved may result in potential side effects on cells[32].

Other than MRI, fluorescence imaging can also be used as a technique for tracking MSCs in tissue engineering since it offers superb temporal and spatial resolution, high sensitivity and is easily applicable. Fluorescence imaging allows insights into living organisms without the need for dissection as a sensitive, cost-effective and non-invasive method[31, 35-39].

In order to achieve fluorescence imaging, a lot of fluorophores have been widely used owing to their high fluorescence quantum yield, relatively low toxicity, and structural versatility that enables facile tuning of the optical properties and conjugation with biomolecules[40-44]. For example, Alexa Fluor ${ }^{\circledR}$ family of 
fluorescent molecules has been commercially available and used as cell and tissue labels in fluorescence microscopy and cell biology. Another example is 4',6-diamidino-2-phenylindole, denoted as DAPI, which has been a commonly used fluorescent probe for selectively labeling of DNA in cell nuclei.

Despite these advantages, most organic fluorophores suffer from their poor photostability, i.e. the fluorescence intensity decays quickly (tens of seconds) under continuous exposure to light. This phenomenon, also called photobleaching, limits the ability of employing fluorescence microscopy or spectroscopy for long-term tracking of biological activities.

Apart from available organic dyes, researchers also use genetic transfection to introduce the gene which can express fluorescent substances to achieve fluorescence labeling, such as green fluorescent protein (GFP). Unfortunately, the commonly used traditional organic or genetically encoded fluorophores, often have broad excitation and emission spectra, susceptible to photobleaching which restrict their applications in long-term and multiplexing studies. Also, genetic transfection is time-consuming which results in low labeling efficiency. Moreover, it has been reported that fluorescent protein transfection even influences the normal cell function, thus hindering the applications of GFPbased probes[45].

\subsection{Nanoparticle based probes}

To overcome the limitations of organic dyes and genetic proteins, various nanoparticles based fluorescent probes have been developed for stem cell tracking, especially semiconductor nanoparticles represented by quantum dots, silica nanoparticles, polymeric nanoparticles, and so on.

\subsubsection{Quantum dots}

In the past decades, new fluorescent probes with high quantum yields and wide absorption/emission spectra have been developed, which are called Quantum dots (QDs). As novel semiconductor NPs, they have attracted numerous 
attention in various applications with superb optical properties[46-50]. They have large effective Stokes shifts. The quantum yield and molar extinction coefficients are high ( 10-100 times of that of organic dyes). The molar extinction coefficient of QD emitted at $510 \mathrm{~nm}$ even if at the lowest absorption wavelength, $450 \mathrm{~nm}$, is greater than that of rhodamine red at its maximum absorption[46] (150,000 versus 129,000 $\mathrm{M}^{-1} \mathrm{~cm}^{-1}$ ) (Figure 2A and B). Most importantly, they can be excited using one single excitation light source but have narrow and symmetric fluorescence spectra ranging from UV to nearinfrared light, allowing to excite QDs with various emissions at the same time[51]. Therefore, QDs can eliminate autofluorescence through selecting a proper excitation wavelength $[52,53]$. Furthermore, their fluorescent emission can be tunable with core size (Figure 2C), making it possible to control the probe color by adjusting the size and separate different probes without spectral overlap[46, 52]. Traditional organic fluorescences often encounter with the serious problem of photobleaching, thus failing to provide long-term stability and achieve multiplex labeling. QDs can tackle these issues by virtue of their good photostability[53]. All the distinctive properties make QDs popularly investigated in biological sensing and imaging.

In addition, QDs can also be conjugated to some biomolecules (proteins, DNAs) or antibodies to enhance biocompatibility. It was reported that human MSCs can be labeled with QDs which have been conjugated with arginine-glycineaspartic acid peptide and differentiated into chondrogenic, androgenic, and adipogenic cells for a long-term tracing probe[30]. Xingyong $\mathrm{Wu}$ et al developed some specific immunofluorescent probes by surface modification and conjugation QDs with IgGs[53]. In the single cell level, they detected the dynamic fluorescence change and compared QD-608 to Alexa-488[53]. The fluorescence of Alexa 488 reduced fast and became dim within $2 \mathrm{~min}$, nevertheless QD $608 \mathrm{kept}$ their brightness for the entire time period (3 min) (Figure 3). 
QDs have exhibited superb photostability and brightness in biological applications, however, the current popularly applied QDs such as CdSe and CdTe have heavy metal elements, which result in the toxicity in oxidative conditions. What's more, some reports indicate QDs may aggregate within cells and exhibit unstable fluorescence signals. These shortcomings compromise the QDs' application in the clinic[30,34].
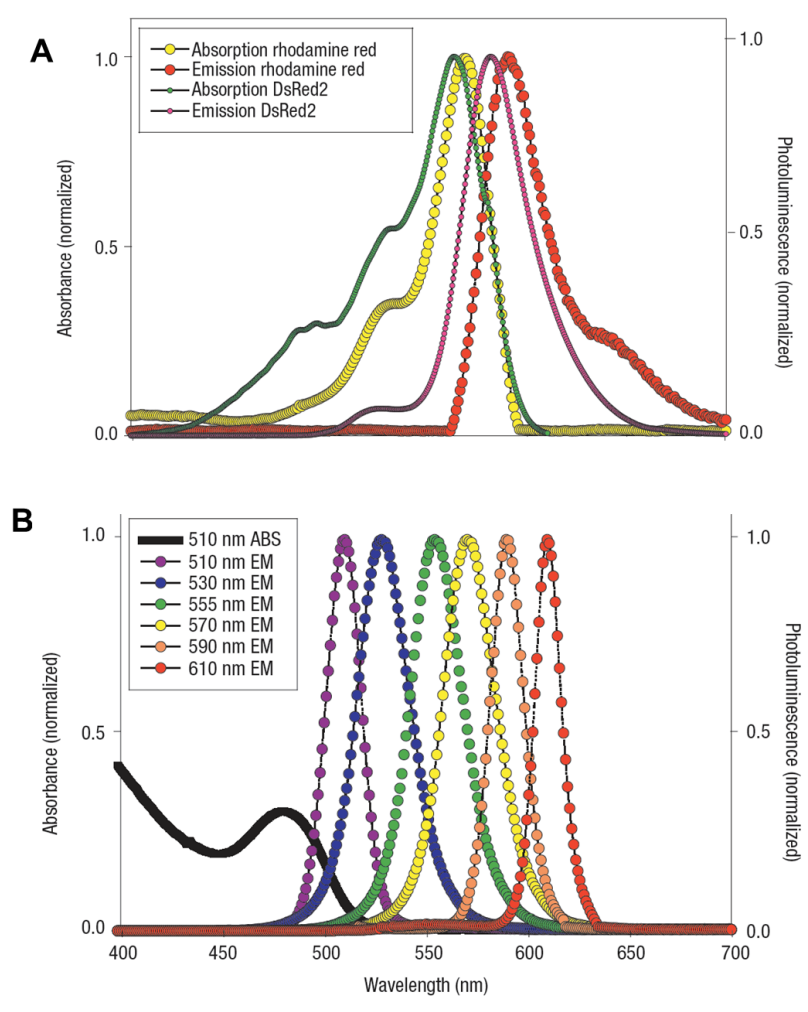

C

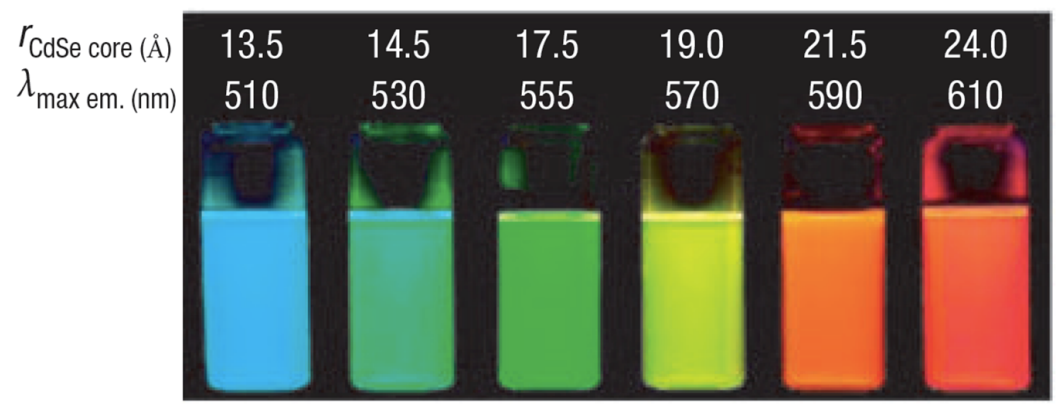


Figure 2. (A) Rhodamine red and genetically encoded DsRed2 protein's absorption and emission. (B) Six QDs' absorption and emission spectra with the black line indicating the absorption of QDs emitted at $510 \mathrm{~nm}$. (C) Six QDs exhibit size-tunable fluorescence properties. Reprinted by permission from Macmillan Publishers Ltd: [Nature Materials][54], copyright (2005).

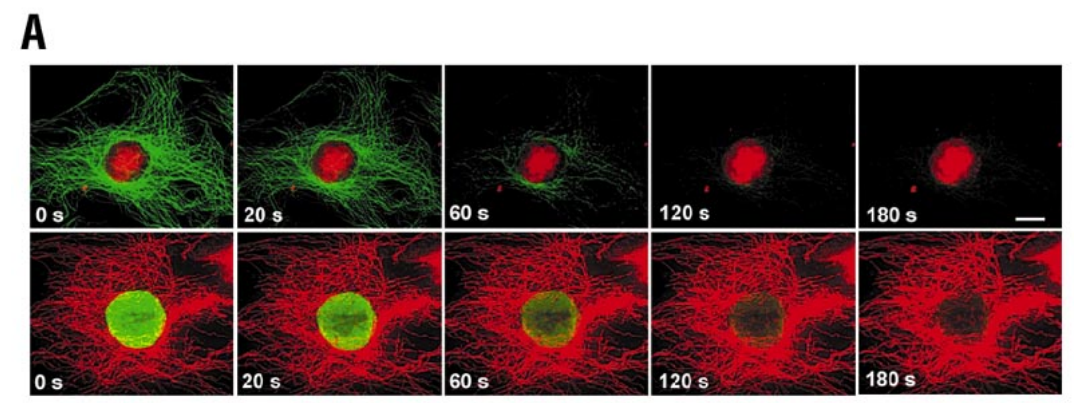

B

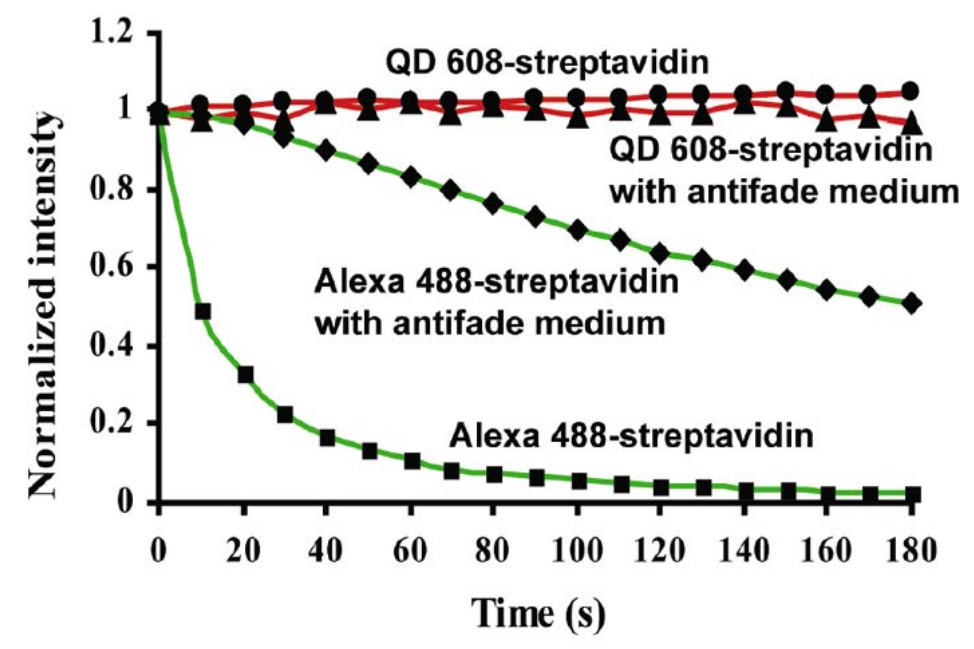

Figure 3. Photostability of QDs compared with Alexa 488 within single cell. (A) In the up row, QD-630-streptavidin (red) stained the nuclear antigens, while Alexa 488 (greeen) labeled microtubules in a 3 T3 cell. In the below row, QD-630-streptavidin (red) stained microtubules, while Alexa 488 (green) labeled the nuclear antigens. During successive exposure to light from a usual mercury lamp $(100 \mathrm{~W})$ for $3 \mathrm{~min}$, the samples were taken images at indicated time points. Scale bar, $10 \mu \mathrm{m}$. (B) Normalized fluorescence intensity change curves of QD-608-streptavidin and Alexa 488 which have stained microtubules and nuclear antigens respectively. Reprinted by permission from Macmillan Publishers Ltd: [Nature Biotechnology][53], copyright (2003).

\subsubsection{Silica nanoparticles}


In addition to quantum dots, researchers also designed many other nanoparticles based on silica and polymers containing fluorescent dyes to improve the photostability. The biocompatible silica shell can protect the concentrated fluorescent dyes from oxidation or decomposition, thus generating strong fluorescence. Especially, the mesoporous silica NPs have attracted much attention as a novel biomaterial since they are biocompatible and biodegradable[55], can provide an adequate surface area and abundant pore volumes[56]. In addition, the silanol groups on the surface can be used for chemical modification for specific function[57].

There are two methods sol-gel or reverse microemulsion to fabricate fluorescent silica nanoparticles[58]. The famous Cornell dots or C-dots have been regulated by FDA for application in tumor and entering phase-I clinical trials. In this example, fluorescent organic dye was conjugated with a silica precursor to fabricate the dye-containing core, followed by co-condension with the silica solgel monomers in a specific order. The fabricated C-dots exhibited strong brightness, photo-stability, and multi-functions on the surface[59].

In the applications of silica nanoparticles on stem cells, Chung et al found that the mesoporous silica nanoparticles containing fluorescein isothiocyanate can be internalized in human bone marrow mesenchymal stem cells, and they did not influence stem cells' viability and proliferation[60]. However, importing the mesoporous silica nanoparticles increased actin polymerization but induced not enough osteogenic signals in human mesenchymal stem cells[61].

\subsubsection{Polymeric nanoparticles}

Similar as silica nanoparticles, polymer-based platform can also provide biocompatibility for the stem cell tracking. In order to achieve fluorescence, there are two methods to contain fluorescent organic dyes, including physical encapsulation in the polymer solution before the fabrication of nanoparticles, and covalent conjugation with the polymer chain. 
Currently, polystyrene (PS) NPs are popular fluorescent polymeric nanoparticles, which mainly encapsulate fluorescent dyes physically in the polymer solution. PS NPs can label stem cells by intracellular uptake by endocytosis and different surface functional groups have influences on cellular uptake. For example, Jiang et al. studied the uptake of two types of polystyrene NPs in MSCs by confocal fluorescence microscopy and flow cytometry, including plain PS NPs and amino-functionalized PS NPs (NPS)[62]. They found NPS nanoparticles could be rapidly internalized and accumulated to a much higher level in MSCs than plain PS nanoparticles, which was predominated by the clathrin-mediated endocytosis[62].

After intracellular internalization, PS NPs can be used as a tracer to track MSCs differentiation. In a further study, Andrea Tautzenberger et al applied phosphonate-functionalised polystyrene nanoparticles fabricated by miniemulsion polymerisation in the differentiation of human MSCs[63]. They have successfully intracellularly incorporated the nanoparticles into the MSCs without using any transfection agents[63]. They demonstrated that these polystyrene nanoparticles didn't affect cell viability and their potential for osteogenic, adipogenic and chondrogenic differentiation[63].

In addition to MSCs, PS NPs can also be applied to ESCs. It was found the ESCs exhibited columnar and embryoid body shape in low concentration (0.4 million/mL) polystyrene nanoparticles[64]. However, ESCs cultured in high concentration (12.2 million $/ \mathrm{mL}$ ) nanoparticles became fibroblast-like and less squamous types[64]. After $24 \mathrm{~h}$, the viability of ESCs was reduced by $40 \%$ and $30 \%$ in the low and high concentration of nanoparticles, respectively, which implies the negative effect of polystyrene nanoparticles on ESCs[64].

In spite of the wide applications, PS NPs have some limitations which include low dye encapsulation and inadequate protection for the dye, leading to easy leaching, quenching and photobleaching[65]. In addition to PS, there are other polymers for the similar applications including poly(thiphene-3-yl-acetic acid), 
poly(arylenediethynlenes), polyacetylene, and so on[65]. However, current available polymer NPs mostly encapsulate dyes in the polymer solutions, while there are few polymeric NP products which are by covalent conjugation. Actually, extinction coefficients (typically $10^{6}-10^{7} \mathrm{M}^{-1} \mathrm{~cm}^{-1}$ ) and quantum yield (up to $80 \%$ ) are generally high in the conjugated polymers, since $\pi^{*}$ excited states can make excitons to easily diffuse within a polymer chain, which enhances the fluorescence responses.

\subsection{Keloid scar treatment by transdermal delivery}

In addition to stem cell tracking, there is another tough problem in the regenerative medicine field, which is abnormal scar treatment, including keloid scars. These scars are formed due to exuberant healing response[66]. The abnormal healing responses include three stages, inflammation at the first 10 days, proliferation from 10 to 14 days and maturation or remodeling from two weeks to years[67]. Keloids as elevated fibrous scars, often progress and exceed the borders of the original wound. Surgery removal has limitations as the keloid scars usually recur.

Keloids are easier formed in dark skin with a 15 - to 20 -fold increased risk probably due to abnormal melanocyte stimulating hormone. People with black or yellow skins have more possibility to form keloids than white skins[68]. In addition, formation of keloids is related with age, and people younger than 30 years old have higher risk, especially teenagers between 10 to 20 years old[69]. Positions in the body including sternal skin, cheeks, earlobes, and shoulders have more tendency to develop keloids[70].

Family genetic reason is recognized to influence the formation of keloid scar[71]. Besides, some accidents including trauma, delayed healing longer than three weeks, and burn, increase keloid development. Also, certain reasons like vaccinations especially bacille Calmette-Guérin vaccination, acne, ear piercing, lacerations, and biopsy procedures can cause abnormal scarring. Acne keloids 
are pretty popular among them. Keloids can cause a lot of trouble to the patients, including not only cosmetic disfigurement and serious emotional distress but also pruritus and pain.

\subsubsection{Conventional treatment for keloid scars}

As mentioned, keloid scars can cause much trouble to the patients. Unfortunately, although various methods were developed to help management of keloid scars, there is still not an ideal therapy which can provide the best treatment for keloids. The main reason for the challenging clinical problem is the lesion often recur at a high rate even after almost any type of available treatment. Currently, the clinical doctors can only check family history or previous problems of scarring and estimate the risk of developing a scar before any surgical procedures. In some cases, doctors have to perform the surgery to remove the scars. Then the doctors have to inject immediately corticosteroid and pressure dressings to enhance wound healing and reduce the skin tension, which is helpful to reduce the risk of scarring[72, 73]. These common measures may cause pain, infection, and high cost. In addition, these available clinical measures require exemplary adherence and follow-up.

One popular method for keloid management, such as orticosteroid injection can suppress inflammation/mitosis as well as increase vasoconstriction of the scar. In a common practice, $10-40 \mathrm{mg} / \mathrm{mL}$ of triamcinolone acetonide suspension (Kenalog) is injected intralesionally. This manner can finally have effects on 50 to $100 \%$ of keloids, however, it has a high recurrence rate (9 to 50\%) and causes a lot of pain[70]. In order to achieve better effects, corticosteroid injections are often combined with surgery to fresher keloids, which are more sensitive to treatments. However, it may result in atrophy, telangiectasias, and hypopigmentation as side effects. In addition to corticosteroid, intralesional injections of verapamil, fluorouracil, and bleomycin are also investigated for keloid scar treatment. For example, fluorouracil $(50 \mathrm{mg} / \mathrm{mL}, 2$ to 3 times per week) injections intralesionally exhibited shrinking keloids safely and didn't 
cause the side effects about tissue atrophy and telangiectasia, which are commonly caused in corticosteroid injections[74].

In addition to intralesional injections of drugs, the second method for the prevention and treatment of keloids is silicone sheeting noninvasively. The mechanism is because silicone sheets are able to soften and flatten the scars through altering the temperature, hydration, as well as the oxygen tension in the obstructed scar[69]. However, this method can only be used for the closed skins not for any open wounds. Although more than 60 related products are commercial, most of them are expensive. And these silicone sheets must be put on the scar regions for 12 to 24 hours every day and last for 2 to 3 months in order to achieve effectiveness, which causes a lot of comfortlessness[67]. Most importantly, a recent review indicated that most researches regarding the promising results about this method have limited quality and subjective bias[75].

In addition, combination of several methods can be applied to achieve a better treatment. In order to increase the reduction of recurrence, the surgical removal of scar is better complete, or only a little left on the wound margin. An effective result was achieved in one study by a combination comprising of surgery, intrakeloidal steroid injection and cream, and silicone gel sheeting. This triple therapy treated 180 patients and took 2 to 4 years for the follow-up. The final results from 120 patients demonstrated the recurrence rate was only $12.5 \%$ after 13 months[76], with 15 out of 120 patients still have keloid formation. The result is promising, but this triple therapy is time consuming and tedious. At first, 180 patients were involved in this study, however, sixty of them discontinued for the follow-up and only 120 patients came for review finally. Thus, this frequent follow-up caused inconveniences to patients, and it will be better there is a method which patients can apply by themselves and don't need to go to hospitals frequently. 


\subsubsection{Keloid fibroblasts engineering by transdermal delivery}

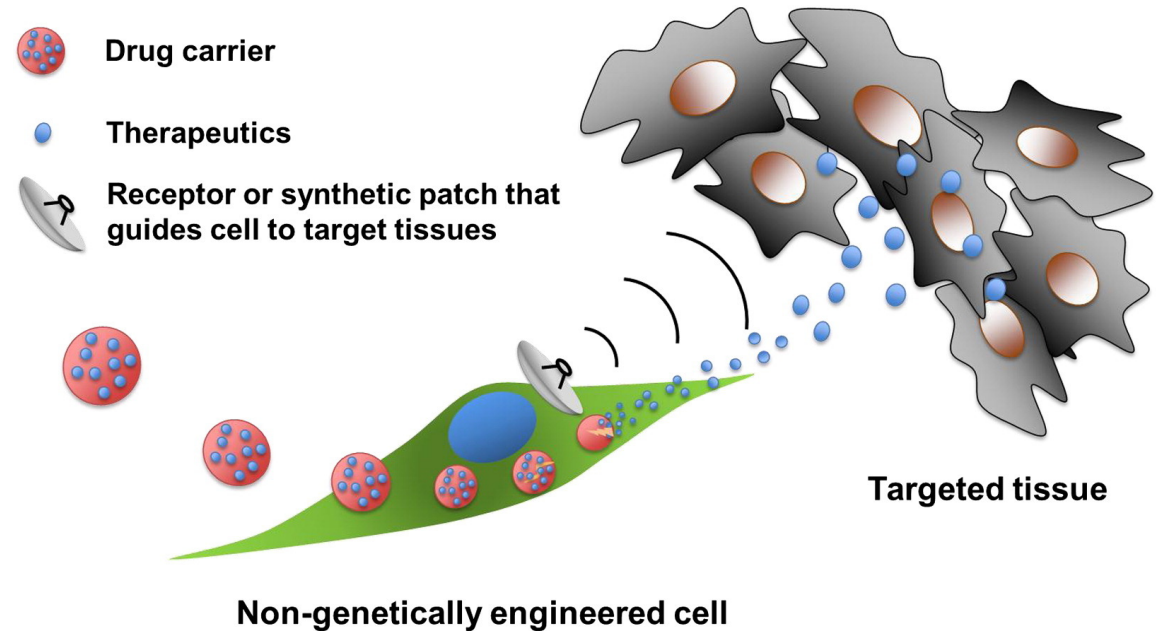

Figure 4. Non-genetic cell engineering. (Reprinted from [77], copyright (2015) with permission from Elsevier)

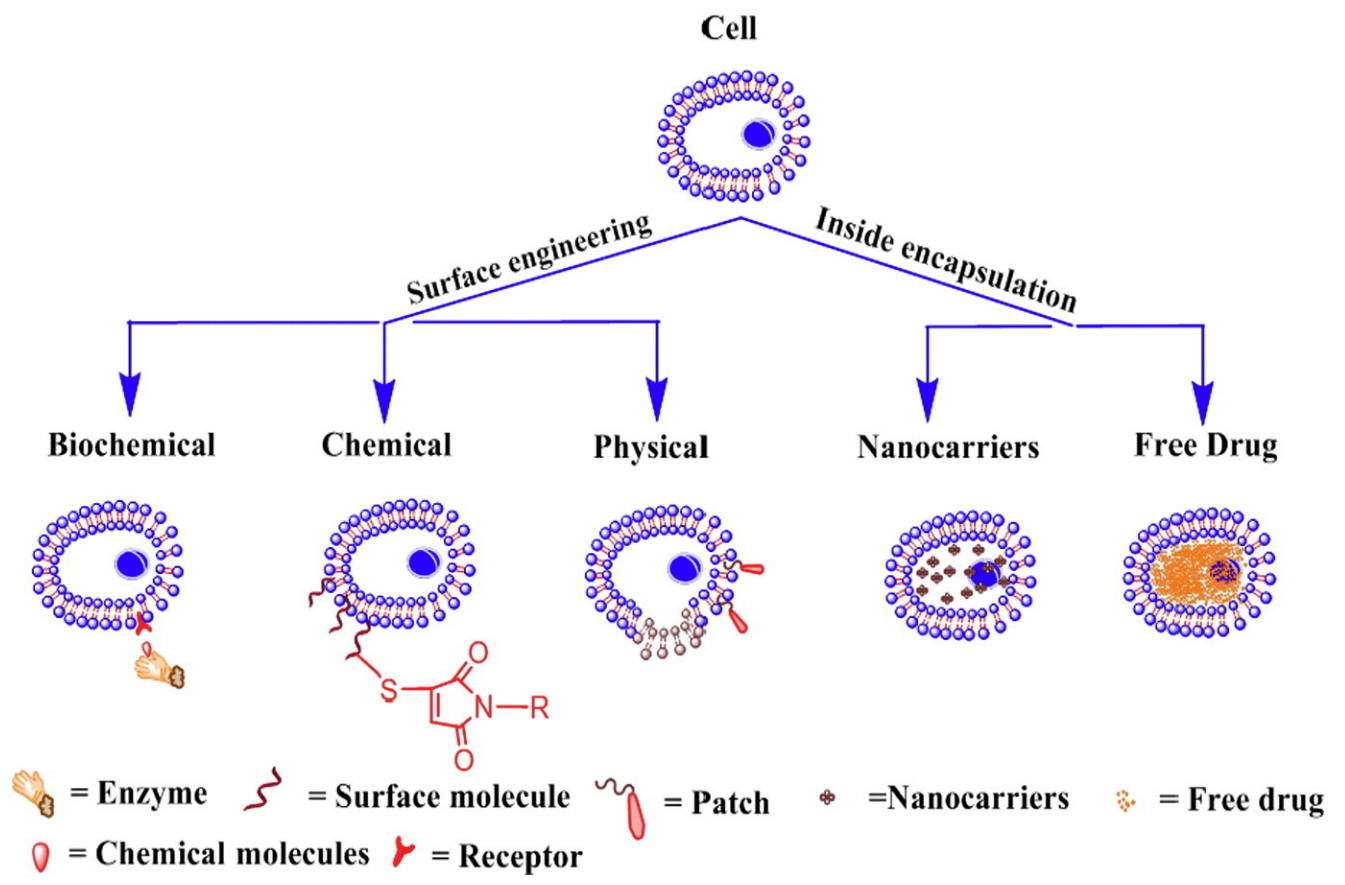

Figure 5. Non-genetic cell engineering strategies including cell surface modification and delivering free drugs or encapsulated drugs by nanocarriers. (Reprinted from [77], copyright (2015) with permission from Elsevier)

In recent years, non-genetic cell engineering (Figure 4) have been extensively explored to achieve new therapeutic function by utilizing physical, chemical and material methods[77]. Compared with genetic engineering methods, these 
non-genetic ways can overcome the concern of safety due to the lack of control over the genomic location of the inserted genes and the risk of insertional mutagenesis. Figure 5 shows the currently available major non-genetic cell engineering strategies. For example, through using drug delivery systems, free drugs can be delivered to targeted tissues. Using non-genetic engineering strategy can deliver a broad range of drugs, including synthetic drugs that cannot be produced by cells through genetic engineering.

In order to effectively inhibit keloid scarring and improve treatment, various drugs as mentioned in Section 1.5.1 have been injected into the keloid lesion by traditional needles. This traditional route causes a lot of pain to the patients, and may have the risk of infection. In addition, it also generates dangerous medical waste and needle re-use especially in developing countries may result in risk of disease transmission. Besides, as these treatments require frequent follow-up in the hospitals, which caused inconveniences to patients.

A novel technology to deliver drugs transdermally by microneedles has achieved great progress in recent years[78, 79]. Transdermal delivery has many advantages compared with the traditional hypodermic route and oral route. It's non-invasive and can be self-administered by patients, and also it can control the release of drugs for a long period of time (up to one week), which could improve patient compliance[78]. In addition, the microneedles with controllable depths can penetrate the skin stratum corneum and avoid the blood and nerve supply inside the skin, which don't cause any pain and infections to the patients, so this delivery method has better compliance. Finally, the transdermal delivery system is generally inexpensive. Currently, this transdermal delivery route has successfully been applied for various drugs, especially delivery of vaccines[78]. The common drugs as mentioned in Section 1.5.1 including for prevention and treatment of keloids, such as corticosteroid have adverse effects. In recent years, more researches clarifying the relationship between intercellular communication mediated by gap junctions and scarring provide new insights 
for improvement for scar reduction. Gap junctions are formed by intercellular channels composed of connexin proteins, which are known to impact inflammatory response, would closure, and scar formation[80-84]. Suppressing connexins activity by antisense oligodeoxynucleotide was found to reduce the spread of tissue damage, accelerate wound closure, and reduce scarring[85].

In order to inhibit connexins activity, various inhibitors were developed including halothane, octanol, 18 $\alpha$-glycyrrhetinic acid, carbenoxolone, and flufenamic acid. However, they have limitations with radical changes in membrane fluidity and influence cell's metabolic/signaling cascades[86]. And all of them exhibited poor specificity towards connexin $(\mathrm{Cx})$ channels[86]. It's urgently required to have a tool that specifically target $\mathrm{Cx}$ channels by a rapid and easily reversible mode of action.

Connexin mimetic peptide, can mimic specific amino acid sequences of two well-conserved extracellular loops of connexins and interfere with their functions[87]. Gap 26 and 27 are the most popular mimetic peptide, with Gap 26 has better efficacy at the same concentration, and have been used extensively to suppress gap-junctional communication constructed of $\mathrm{Cx} 43$ mainly, but also Cx32, 40 and 37 in a wide range of cells and tissues[87].

\subsection{Objectives of this dissertation}

Tracking the cell movement, location, migration, and distribution of transplanted stem cells can help instruct the optimum cell dosage, transplantation time, and evaluate efficacy. Currently available tracking probes suffer from poor photostability or potential toxicity. It is very necessary to develop a photostable probe with excellent biocompatibility to track the MSCs for a long-term without affecting the cell viability, proliferation, and differentiation potency.

In another issue for treatment of keloid scars to achieve skin regeneration, traditional treatments including surgery removal, silicone sheet dressings, 
hypodermic injections of drugs like corticosteroid, fluorouracil (5-FU), may result in pain, infections, and adverse effects as well as less patients' compliance. It's imperative to deliver drugs in a cheap, non-invasive and convenient way to patients without causing pain and infections.

This thesis aims to solve the above issues by developing and applying biocompatible and photostable fluorescent nanoparticles for long-term stem cell tracking and engineering keloid fibroblasts by transdermal delivery of hydrophilic peptide for keloid scar treatment.

More specifically, this thesis targets to achieve the following objectives:

1) To enhance the photostability of organic fluorophores, for example, di(thiophene-2-yl)-diketopyrrolopyrrole (DPP), it was covalently linked in the middle of a biocompatible and bioresorbable polymer chain, polycaprolactone (PCL). The polymer chain protection and steric effect are expected to suppress the aggregation of DPP to increase photostability. Subsequently, the polymer will be used to fabricate nanoparticles and applied for mesenchymal stem cell imaging. The nanoparticles' photostability will be compared in Chapter 2 with commercial organic dyes, well-recognized photostable quantum dots, and physical blends of dye/polymer complexes.

2) In order to efficiently track mesenchymal stem cells differentiation, the fluorescent probes should not only have strong fluorescence and are highly photostable, but also cause little negative effects on stem cell viability, proliferation behaviors, as well as differentiation performance. The second goal is to investigate the applications of the developed PCLDPP-PCL nanoparticles in long-term tracking of MSCs differentiation. The effects on cell viability, proliferation, and multipotency of the MSCs in the three lineages in vitro including osteogenic, adipogenic and chondrogenic differentiations will be explored in Chapter 3 . 
3) In another target to develop a transdermal delivery system for keloid scar treatment, (polyethylene glycol) diacrylate (PEGDA)-based microneedles with good biocompatibility will be fabricated through photo-polymerization in Chapter 4. A model hydrophilic peptide, gap junction inhibitor (Gap 26), will be loaded into PEGDA microneedles through the swelling effect in the aqueous solution. Then the effects of peptide on gap junction based intercellular communication will be investigated and the delivery system will be applied to a keloid scar model and detected its therapeutic effects. 


\section{Chapter 2: Mesenchymal Stem Cell Imaging by Fluorescent and Bioresorbable Polymeric Nanoparticles with Enhanced Photostability}

\subsection{Introduction}

Fluorescence imaging, due to its high specificity, sensitivity and imaging contrast, has proven to be an indispensable tool in modern biotechnology for advancing our understanding of various biological systems[44, 88]. Among a variety of probes for fluorescence imaging, small organic fluorophores have been most widely used owing to their high fluorescence quantum yield, relatively low toxicity, and structural versatility that enables facile tuning of the optical properties and conjugation with biomolecules[40-44]. For example, Alexa Fluor ${ }^{\circledR}$ family of fluorescent molecules has been commercially available and used as cell and tissue labels in fluorescence microscopy and cell biology. Another example is 4',6-diamidino-2-phenylindole, denoted as DAPI, which has been a commonly used fluorescent probe for selectively labeling of DNA in cell nucleus.

Despite these advantages, most organic fluorophores suffer from their poor photostability, i.e. the fluorescence intensity decays quickly (tens of seconds) under continuous exposure to light. This phenomenon, also called photobleaching, limits the ability of employing fluorescence microscopy or spectroscopy for long-term tracking of biological activities. Previous studies indicate that the formation of non-fluorescent triplet states (denoted as $T_{1}$ ), following the photonic excitation process, and the presence of molecular oxygen play a major role in the photobleaching of organic fluorophores. In air-saturated solutions, reactions between molecular oxygen and $T_{1}$ lead to substantial generation of reactive oxygen species (ROS), including $\mathrm{HO} \bullet, \mathrm{HO}_{2} \bullet$, and $\mathrm{H}_{2} \mathrm{O}_{2}$, and rapid photobleaching. 
Several strategies such as oxygen depletion and addition of protective agents as triplet-state quenchers, although being able to reduce photobleaching to some extent, have achieved limited success in fluorescence-based bioimaging[44]. Recently, Blanchard and coworkers[32] have reported a strategy of covalently linking protective moieties (e.g. cyclooctatetraene, 4-nitrobenzyl alcohol, 6hydroxy-2,5,7,8-tetramethylchroman-2-carboxylic acid) to cyanine fluorophores to enhance the photostability. Single-molecule fluorescence imaging showed remarkable enhancement of photostability in these modified fluorophores compared to the native ones without protective agents. Nevertheless, the improvement of photostability varies with different members of the cyanine class, suggesting the need for a deeper understanding of how the protective agents provide photostabilization[32]. Relatively high photostability has been observed in inorganic semiconductor quantum dots[50, 89-92], organic dye-silica core/shell $\mathrm{NPs}[38,93]$, some organic and polymeric fluorescent dots[39, 94], and some optimized fluorescence proteins through mutagenesis[95]. For example, enhanced photostability has been recently reported in nanoparticles composed of organic fluorophores with aggregation induced emission (AIE) effect[94, 96-98], but such improvement has been only observed in limited groups of organic dyes and polymers. Despite these recent advances, a general strategy of obtaining highly luminescent, photostable and non-phototoxic fluorophores for long-term bioimaging is still in great demand[44].

Herein we report a facile strategy of enhancing the photostability by covalently linking an organic fluorophore, di(thiophene-2-yl)-diketopyrrolopyrrole (DPP), in the middle of a biocompatible and bioresorbable polymer chain, polycaprolactone (PCL) (Fig. 1a). This polymer, denoted as PCL-DPP-PCL, has a number-average molecular weight $\left(M_{\mathrm{n}}\right)$ of 38,300 Da and a polydispersity index $\left(M_{\mathrm{w}} / M_{\mathrm{n}}\right)$ of 1.98. In contrast to small-molecule DPP derivatives which often show dramatic fluorescence quenching in solid states due to significant intermolecular $\pi-\pi$ aggregation, we found that PCL-DPP-PCL tethered with 
long PCL chains shows remarkably bright fluorescence in solid states, suggesting that the aggregation of DPP moieties is suppressed by the covalently attached PCL chains. In addition, PCL as a biocompatible and bioresorbable polyester has attracted much attention for use as an implantable biomaterial in tissue engineering and drug delivery[99]. Biodegradation of PCL is slow compared to other polymers, thus making it suitable for long-term delivery extending over a period of more than one year.

We expected that the integration of strong fluorescence of DPP with the biophysical properties of PCL could make PCL-DPP-PCL an ideal fluorescence probe for bioimaging. Considering the hydrophobic nature of both DPP and PCL in this polymer, we used Pluronic ${ }^{\circledR} 127$ as a surfactant and followed a method of nanoprecipitation to disperse PCL-DPP-PCL as nanoparticles (NPs) in aqueous media (Figure 6a). The resulting NPs were highly fluorescent and showed remarkably enhanced photostability compared to commercial organic fluorophores in imaging of both human liver cancer cell line (HuH-7) and porcine mesenchymal stem cells (MSCs). Moreover, these polymeric NPs showed brighter and more robust fluorescence properties compared to commercially available imaging probes such as quantum dots $\left(\right.$ Qtracker $\left.^{\mathbb{R}}\right)$ in long-term tracing of cells.

\subsection{Materials and Methods}

\subsubsection{Materials and reagents}

Dulbecco's Modified Eagle's Medium (DMEM), phosphate buffered saline (PBS), penicillin/streptomycin antibiotics, Alexa Fluor ${ }^{\circledR} 633$ phalloidin, TrypLE ${ }^{\text {TM }}$ Express Enzyme $(1 \times)$, PrestoBlue cell viability reagent, fetal bovine serum (FBS), and Qtracker ${ }^{\circledR} 705$ Cell Labeling Kit were ordered from Life Technologies (Singapore). Other reagents including Bovine Serum Albumin (BSA), formalin solution, 4', 6-diamidino-2-phenylindole (DAPI) were purchased from Sigma-Aldrich (Singapore). The synthetic details of DPPHT, 
PCL-DPP-PCL, PCL-QA-PCL and the polymer NPs are described in Supporting Information.

\subsubsection{General materials characterization}

Scanning electron microscopy (SEM) was performed on a field emission scanning electron microscopy JSM 6701F (JEOL, Japan) operated at $10 \mathrm{kV}$. Transmission electron microscope (TEM) measurements were carried out on a JEM-3010 (JEOL, Japan) electron microscope operating. UV-vis transmission spectrum was recorded on a UV-2450 (SHIMADZU, Japan) spectrophotometer. Photoluminescence spectra were measured with LS 55 (PerkinElmer, USA) fluorescence spectrometer. The particle size was measured with a dynamic light scattering (DLS) instrument, Zetasizer Nano ZS (Malvern, UK).

\subsubsection{Cell culture}

HuH-7 Cell Line (Hepato cellular carcinoma cells) were obtained from National Cancer Center (Singapore) and cultured in DMEM supplemented with 10\% FBS and $1 \%$ penicillin/streptomycin in the incubator with $37^{\circ} \mathrm{C}$ with $5 \% \mathrm{CO}_{2}$. Mesenchymal stem cells (MSCs) were isolated from porcine bone marrow aspirates following institutional guidelines, which demonstrated good purity and stability[100]. MSCs were also cultured in DMEM with 10\% FBS and 1\% penicillin/streptomycin in the incubator with $37^{\circ} \mathrm{C}$ with $5 \% \mathrm{CO}_{2}$.

\subsubsection{In vitro cellular uptake}

In order to explore the cellular uptake of PCL-DPP-PCL NPs and physically blended PCL/DPP NPs, HuH-7 or MSCs were seeded on Petri dish and maintained in the incubator at $37{ }^{\circ} \mathrm{C}$ with $5 \% \mathrm{CO}_{2}$. Then, NPs were diluted in the medium $(0.2 \mathrm{mg} / \mathrm{mL})$ and incubated with cells at $37{ }^{\circ} \mathrm{C}$ with $5 \% \mathrm{CO}_{2}$ after $12 \mathrm{~h}$, followed by washing excess NPs with $1 \times$ PBS for three times after $24 \mathrm{~h}$. Then formalin solution was used to fix cells for $20 \mathrm{~min}$ followed extensive washing by PBS for three times. Then $0.1 \%$ Triton $\mathrm{X}-100$ was used to permeabilize cells for 5 minutes at room temperature, followed by washing 
twice by PBS. 5\% BSA was used to block unspecific positions for $30 \mathrm{~min}$. Then filamentous actin (F-actin) cytoskeleton was stained by Alexa Fluor ${ }^{\circledR} 633$ phalloidin for $1 \mathrm{~h}$ at room temperature. Finally, nucleus was stained by DAPI for $1 \mathrm{~min}$ after washing. 405, 561, and $633 \mathrm{~nm}$ lasers were used as excitations for DAPI, NPs, and Alexa Fluor ${ }^{\circledR} 633$ phalloidin, respectively. Their emissions were collected through a confocal microscope (LSM 780) with 421-481 nm, 569-621 nm, and 650-758 nm for three individual channels (Objective: LD Plan-Neofluar 20x/0.4 Korr M27). In order to observe cell internalization of PCL-QA-PCL NPs, similar procedures were followed except that 405, 488, and $633 \mathrm{~nm}$ lasers were applied to stimulate DAPI, PCL-QA-PCL nanoparticles, and Alexa Fluor ${ }^{\circledR} 633$ phalloidin, respectively. Their emissions were collected through a confocal microscope (LSM 780) with 421-481 nm, 508-534 nm, and 650-758 nm for the three channels (Objective: LD Plan-Neofluar 20x/0.4 Korr M27).

\subsubsection{Photobleach assay}

In order to perform the photobleach test of PCL-DPP-PCL NPs and physically blended PCL/DPP NPs, HuH-7 or MSCs were plated on a petri dish and maintained in the incubator with $37{ }^{\circ} \mathrm{C}$ and $5 \% \mathrm{CO}_{2}$. NPs were added to the medium after $12 \mathrm{~h}$ and cultured with cells at $37{ }^{\circ} \mathrm{C}$ with $5 \% \mathrm{CO}_{2}$ for $24 \mathrm{~h}$. Subsequently, excess NPs were removed by PBS. Thereafter, cells were fixed and stained with Alexa Fluor ${ }^{\circledR} 633$ phalloidin and DAPI as mentioned above. After being multiply-stained with a cocktail of three dyes, both MSCs and $\mathrm{HuH}-$ 7 were continuously exposed to $405 \mathrm{~nm}, 561 \mathrm{~nm}$, and $633 \mathrm{~nm}$ laser irradiation with a higher intensity than that in cellular uptake assays and the fluorescence emissions from each dye were recorded at specific time points (Objective: LD Plan-Neofluar 20x/0.4 Korr M27). The nominal laser powers were calculated by multiplying the maximum laser power by the percentage of intensity output during photobleaching. In Figure 9 and 12, nominal laser powers of $405 \mathrm{~nm}$, $561 \mathrm{~nm}$, and $633 \mathrm{~nm}$ were $20 \mathrm{~mW}, 20 \mathrm{~mW}$, and $5 \mathrm{~mW}$, respectively. For the photobleach test of PCL-QA-PCL NPs, $405 \mathrm{~nm}, 488 \mathrm{~nm}$, and $633 \mathrm{~nm}$ lasers 
were used (Objective: LD Plan-Neofluar 20x/0.4 Korr M27). In Figure 13, nominal laser powers of $405 \mathrm{~nm}, 488 \mathrm{~nm}$, and $633 \mathrm{~nm}$ were $25 \mathrm{~mW}, 25 \mathrm{~mW}$, and $5 \mathrm{~mW}$, respectively. Images were obtained in every scans and bleaches were repeated after every scans. The time in second indicated on images was the moments for scanning.

\subsubsection{Long-term cell tracing}

HuH-7 tumor cells and MSC were plated in a 6-well plate and maintained in the incubator with $37^{\circ} \mathrm{C}$ and $5 \% \mathrm{CO}_{2}$. Two nM PCL-DPP-PCL NPs and Qtracker ${ }^{\circledR}$ 705 were added after $12 \mathrm{~h}$. After incubation for $48 \mathrm{~h}$, excess NPs were removed away by PBS. Then the cells were trypsinized by TrypLE Express and subcultured into another 6-well plate. After a number of days (Day 1, 3, 5, 7 for HuH-7 and Day 1, 7, 14 for MSC), cells were imaged by fluorescent microscopy (Olympus IX71, excited at 530-550 nm, emission filter 575IF). Finally, the cells were washed by PBS and detached by TrypLE Express. After centrifugation (500 g, 5 min), supernatant were discarded and cell pellets were collected and resuspended in PBS for flow cytometry (LSRII, BD Biosciences). The fluorescence from PCL-DPP-PCL NPs and Qtracker ${ }^{\circledR} 705$ were analyzed by PE (excitation/emission: $561 \mathrm{~nm} /(567 \mathrm{~nm}-597 \mathrm{~nm}))$ and PE-cy5 (excitation/ emission: $561 \mathrm{~nm} /(660 \mathrm{~nm}-760 \mathrm{~nm}))$ channels, respectively. Blank cells without treatment with NPs were set as control. Finally, FlowJo software was used to analyze the data.

\subsubsection{In vitro cytotoxicity}

Cell viability effect of PCL-DPP-PCL NPs with different concentrations in HuH-7 and MSCs was evaluated by PrestoBlue assay. These cells were plated on a 96-well plate with 10000 cells/well and maintained in the incubator with $37{ }^{\circ} \mathrm{C}$ and $5 \% \mathrm{CO}_{2}$ for $12 \mathrm{~h}$. Then, PCL-DPP-PCL nanoparticles with different concentrations were diluted in the medium and incubated for 24 and $72 \mathrm{~h}$. Subsequently, excess NPs were removed away with PBS. Then, PrestoBlue reagent in DMEM were added. Simutaneously, PrestoBlue reagents in DMEM 
only were also put to blank wells as control. Supernatant in each well were transferred into another new 96-well plate in order to reduce the absorbance background of NPs after $1 \mathrm{~h}$. Then, the absorbance at $571 \mathrm{~nm}$ with reference wavelength at $600 \mathrm{~nm}$ was measured by Plate Reader (Tecan Infinite M200 series Pro) and the percentage reduction in each sample was calculated based on the following formula as suggested by the manufacturer.

$\%$ Reduction of PrestoBlue Reagent $=\left[\left(117216 \times \mathrm{A}_{1}\right)-\left(80586 \times \mathrm{A}_{2}\right)\right] /$ $\left[\left(155677 \times \mathrm{N}_{2}\right)-\left(14652 \times \mathrm{N}_{1}\right)\right] \times 100$, where $\mathrm{A}_{1}$ is absorbance of test wells at 570 $\mathrm{nm}, \mathrm{A}_{2}$ is absorbance of test wells at $600 \mathrm{~nm}, \mathrm{~N}_{1}$ is absorbance of media only wells at $570 \mathrm{~nm}$, and $\mathrm{N}_{2}$ is absorbance of media only wells at $600 \mathrm{~nm}$. Blank cells were regarded as control and its cell viability was regarded as $100 \%$. Cell viability treated with a specific concentration of NPs $=(\%$ reduction of PrestoBlue reagent at this concentration) / (\% reduction of PrestoBlue reagent of cells not treated by NPs). Statistics analysis of different groups was conducted by the paired Student's t-test using online calculator (http://www.physics.csbsju.edu/stats/t-test.html). All experiments were repeated in three times. $p$ value of $<0.05$ was considered to be statistically significant.

\subsubsection{2,5-bis(6-hydroxyhexyl)-3,6-di(thiophen-2-yl)pyrrolo[3,4-c]pyrrole 1,4 (2H,5H)- dione (DPPHT)}

Potassium carbonate (8.28 g, $60 \mathrm{mmol})$ and DPP-monomers (3,6-di(thiophen2-yl)pyrrolo[3,4-c]pyrrole-1,4(2H,5H)-dione) (6 g, $20 \mathrm{mmol})$ were mixed in $200 \mathrm{~mL}$ of dimethylformamide under stirring in a $250 \mathrm{~mL}$ three-neck round bottom flask under nitrogen protection. The mixture was heated up to $140{ }^{\circ} \mathrm{C}$ followed by addition of 6-bromo-1-hexanol (10.88 g, $60 \mathrm{mmol})$ dissolved in 25 $\mathrm{mL}$ of dimethylformamide dropwise over 1 hour. The reaction mixture was stirred at $140{ }^{\circ} \mathrm{C}$ overnight. Then the flask was allowed to cool to room temperature and rinsed with chloroform. The product was washed by saturated brine, and extracted by dichloromethane twice. The organic phase was dried with $\mathrm{MgSO}_{4}$. The crude product was further purified by silica chromatography 
(dichloromethane: tertahydrofuran $=1: 1$ ) to obtain dark purple solid DPPHT $(1.02 \mathrm{~g}, 10 \%)$

\subsubsection{Synthesis of PCL-DPP-PCL and PCL-QA-PCL}

The DPP or QA initiator were added into a sealed Schlenk tube with nitrogen protection, and dissolved in anhydrous toluene under magnetic stirring. Stannous octoate as the catalyst was injected through the rubber seal with a syringe. $\varepsilon$-Caprolactone was injected into the reaction mixture. The mixture was degassed with $\mathrm{N}_{2}$-bubbling before the Schlenk tube was submerged in an oil bath and the reaction mixture was heated up to $110{ }^{\circ} \mathrm{C}$ and kept at this temperature overnight. Crude product was purified by dissolved in tetrahydrofuran and precipitated in cold methanol twice.

\subsubsection{General procedure for the synthesis of the PCL-DPP-PCL nanoparticles}

In a typical procedure, $6 \mathrm{~mL}$ of PCL-DPP-PCL $(3 \mathrm{mg} / \mathrm{mL}$ in THF) was injected into $30 \mathrm{~mL}$ of vigorously stirred solution of Pluronic ${ }^{\circledR} 127(8.3 \mathrm{~g} / \mathrm{L}$ in water) at room temperature for 3 mins. Then, the sample was left for stabilization for about 2 hours. Then the sample was dialyzed against DI-water using a $4 \mathrm{kDa}$ membrane. The resulting products were collected by centrifugation at $11,500 \mathrm{~g}$ for $15 \mathrm{mins}$, and the sediments were redispersed in di-water before characterization or dispersed in $1 \times \mathrm{PBS}$ buffer for cell experiments.

\subsection{Results and discussion}

\subsubsection{Fabrication and characterization of NPs.}

Figure 6 indicates the structure of PCL-DPP-PCL and a schematic demonstrating the fabrication of the NPs through a nanoprecipitation process[101-104]. In this process, an aliquot of PCL-DPP-PCL dissolved in tetrahydrofuran (THF) (3 mg/mL) was added to a vigorously stirred solution of Pluronic $^{\circledR} 127$ in water. Hydrophobic interaction of PCL-DPP-PCL drove the self-assembly and the polypropylene oxide (PPO) block of Pluronic ${ }^{\circledR} 127$ which 
collapsed to form the core of the NPs. The shell of the NPs consists of PEG chains originated from Pluronic ${ }^{\circledR} 127$ that extrude to the aqueous media to provide the colloidal stability.

A representative scanning electron microscopy (SEM) image (Figure 6b) shows that the resulting particles appear spherical and uniform in size and shape. A statistical analysis of the particle size gives an average diameter of $51 \pm 4 \mathrm{~nm}$. Assuming a density of $1.14 \mathrm{~g} \cdot \mathrm{cm}^{-3}$ for PCL at room temperature and neglecting the contribution of Pluronic ${ }^{\circledR} 127$ to the diameter of each NP, the estimated average mass of each NP $(d=51 \mathrm{~nm})$ was $4.77 \times 10^{4} \mathrm{KDa}$, and that in average 1,240 polymer chains of PCL-DPP-PCL $\left(M_{\mathrm{n}}=38,300 \mathrm{Da}\right)$ existed in the core of each NP.

While we observed that the size of the NPs could be tuned by changing the concentration of the polymer in THF, the following results and discussion will only focus on NPs with an average diameter of $51 \mathrm{~nm}$, which has been recently demonstrated to be within the optimal size range in monodisperse drug-silica nanoconjugates for deep tissue penetration and high retention in tumors[105]. Figure 6c shows that the internal structure of the PCL-DPP-PCL NPs appeared amorphous as measured with transmission electron microscopy (TEM). In addition, the size and shape of the NPs measured with TEM were consistent with those measured with SEM. Figure 6d shows the result of dynamic laser light scattering (DLS) measurement, which gives an average diameter of 110 $\mathrm{nm}$ for the NPs dispersed in water. The size difference observed using electron microscopy and DLS, which has also been observed for other soft-matter particles, reflects the fact that the polymer NPs existed as a colloidal and swollen state in aqueous media, while they collapse and shrink to smaller sizes after being dried in air and imaged under the high-vacuum condition of electron microscopy. 


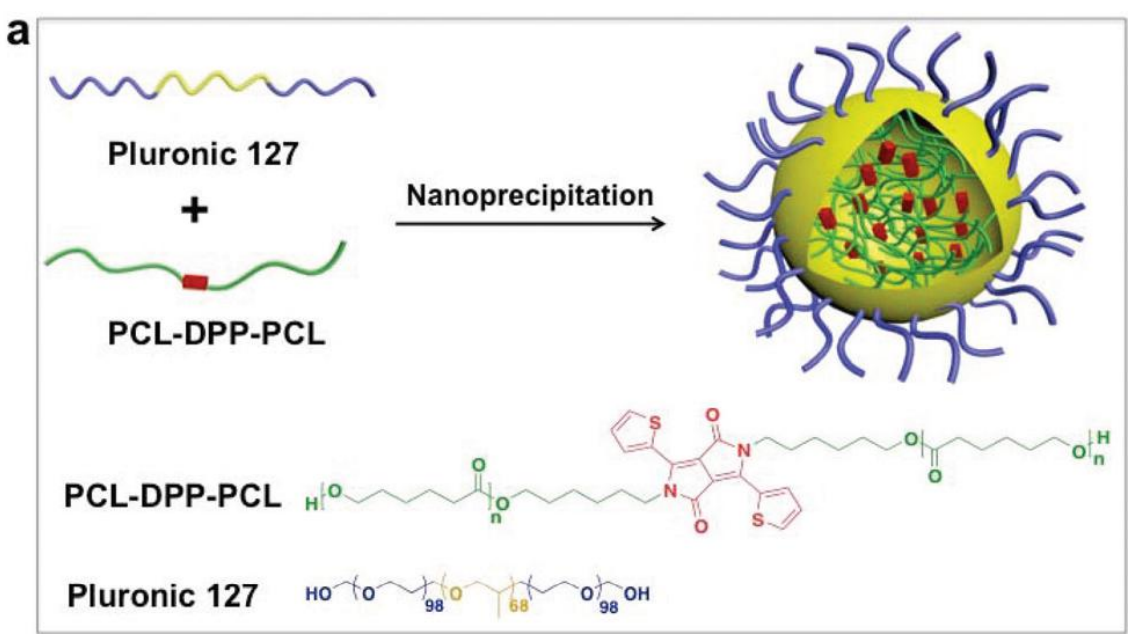

b

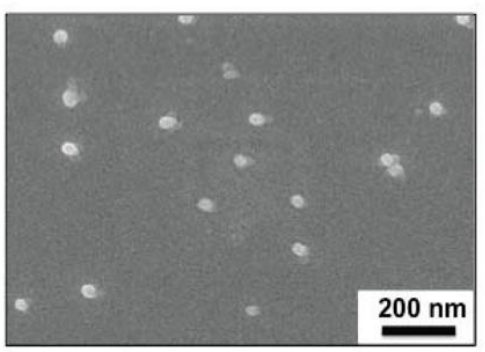

C

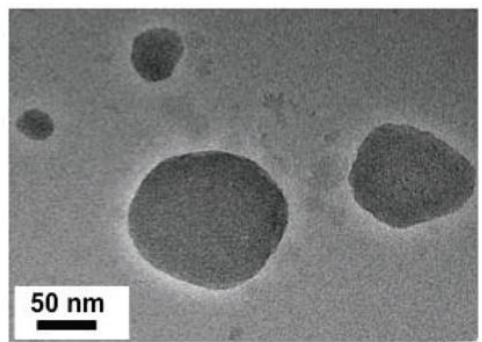

d

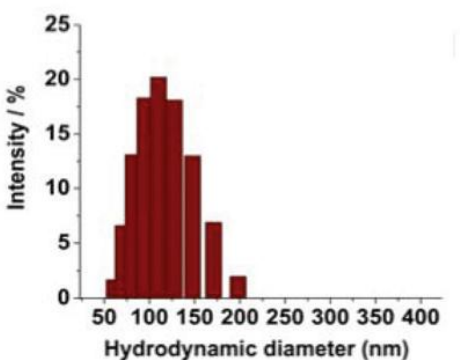

e

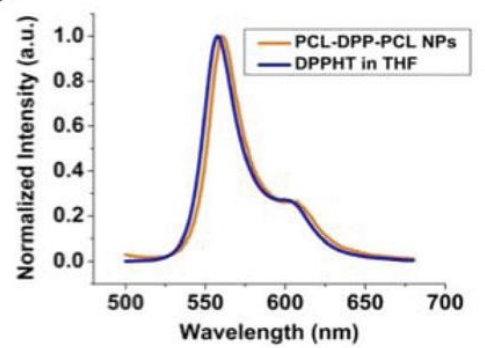

Figure 6. (a) Schematic showing the fabrication process of PCL-DPP-PCL nanoparticles by nanoprecipitation with Pluronic $\AA 127$ as the stabilizer. (b-e) The morphology characterization and optical properties of PCL-DPP-PCL nanoparticles: (b) SEM image showing the average particle size of $51 \pm 4 \mathrm{~nm}$. (c) TEM image of PCL- DPP-PCL nanoparticles from a water dispersion. (d) The dynamic light scattering test shows that the hydrated NPs have an average diameter of $110 \mathrm{~nm}$ in water dispersion. (e) The fluorescence emission spectra of PCL-DPPPCL nanoparticles (orange) and DPPHT in THF (blue) under $488 \mathrm{~nm}$ excitation. (This figure is reprinted with copyright from[106]) 


\section{a}
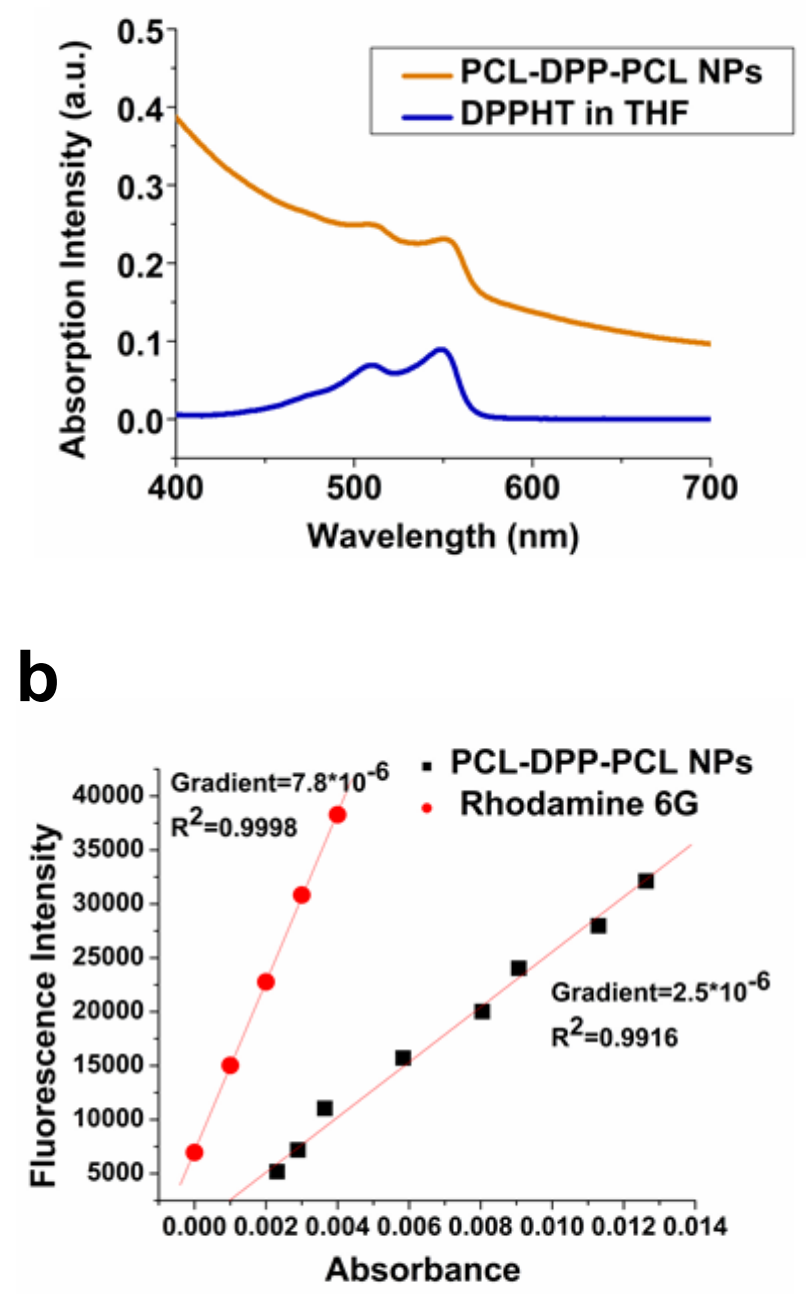

Figure 7. (a) Absorption spectra of PCL-DPP-PCL NPs and DPPHT in THF and (b) linear plots for standard samples and PCL-DPP-PCL NPs. (This figure is reprinted with copyright from[106])

Both the UV-vis absorption (Figure 7) and emission spectra (Figure 6e) of the PCL-DPP-PCL NPs appear similar to those of a small-molecule DPP derivative, [2,5-bis(6-hydroxyhexyl)-3,6-di(thiophen-2-yl)pyrrolo[3,4-c]pyrrole-

1,4(2H,5H)-dione] (DPPHT) in dilute solution of THF. The NP dispersion in water shows characteristic absorption peaks of DPP at 505 and $550 \mathrm{~nm}$, respectively, despite some light-scattering effect (Figure 7). The emission spectrum (Figure 6e) of the PCL-DPP-PCL NP dispersion shows a major 
emission peak located at $560 \mathrm{~nm}$, accompanied with a shoulder at $600 \mathrm{~nm}$. There is a slight red shift $(3 \mathrm{~nm})$ of the maximum emission peak in the NP dispersion compared to that of DPPHT in THF.

The fluorescence quantum yield of the PCL-DPP-PCL NPs in water is as high as 0.29 , based on Rhodamine 6G (quantum yield $=0.95$, in ethanol)[107] as the standard. These results imply that the intermolecular $\pi-\pi$ aggregation among DPP units is minimal in the core of the PCL-DPP-PCL NPs.

\subsubsection{In vitro cellular uptake of NPs}

To explore the potential of these NPs in bioimaging application, we first studied the cellular uptake in vitro. Two types of cell lines were chosen to this end. HuH-7 is a hepatocyte derived carcinoma cell line that has been used as a popular model for cancer research and some transfection study on hepatological infectious diseases.[108-110]

MSCs are multipotent stromal cells that are able to differentiate into a variety of important cell lineages, and have become a promising cell source for regenerative medicine and tissue engineering.[25] Therefore we believe these two types of cells are both representative and biomedically relevant to characterize the prepared PCL-DPP-PCL NPs for cell imaging. Figure 8 shows the fluorescence images of these two types of cells observed from three channels corresponding to different fluorophores: blue (DAPI), yellow (PCL-DPP-PCL NPs), and red (Alexa Fluor ${ }^{\circledR} 633$ phalloidin). 


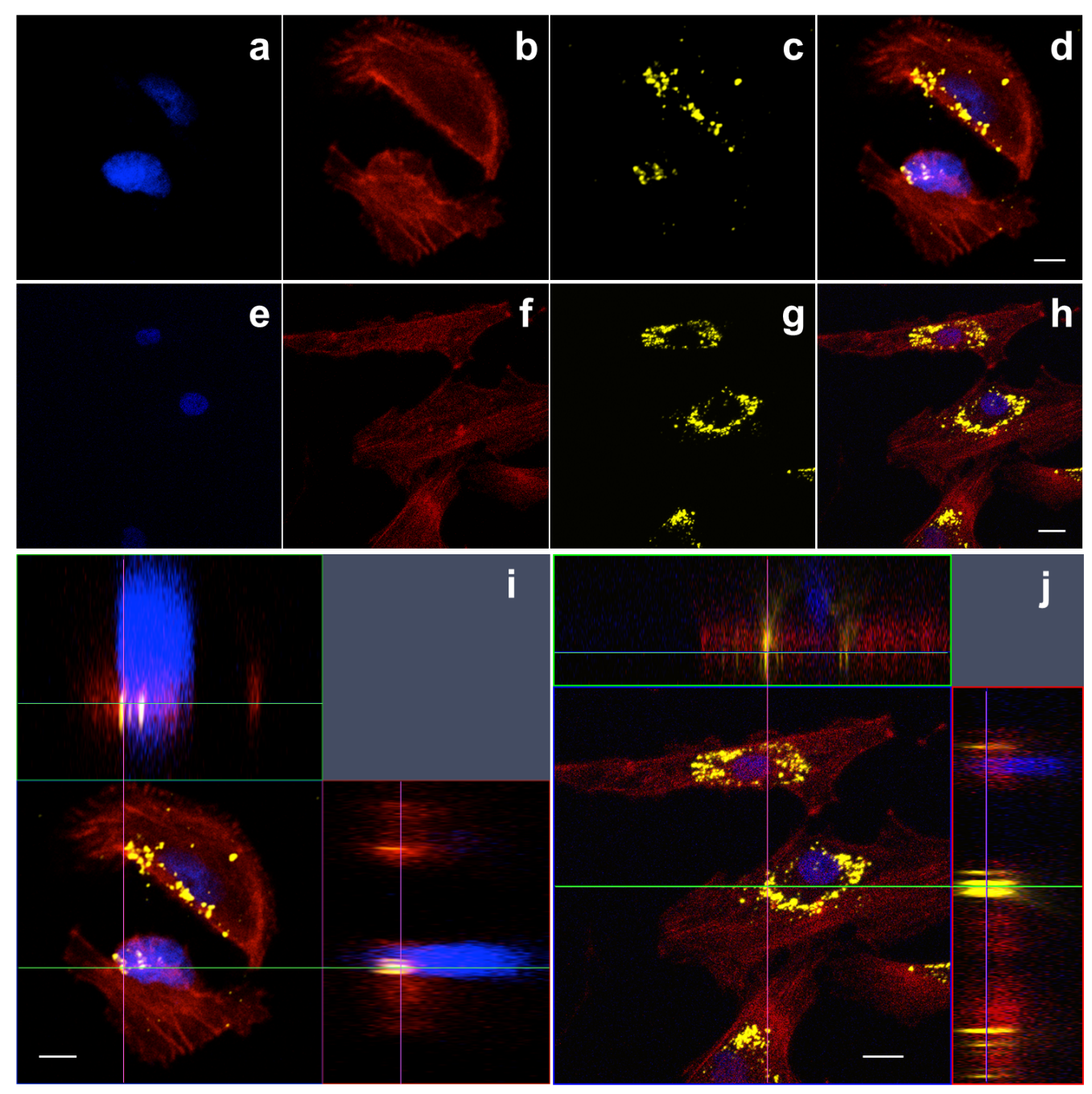

Figure 8. Cellular uptake of PCL-DPP-PCL nanoparticles (NPs) in HuH-7 tumor cells (a-d and i) and MSCs (e-h and j) imaged by confocal laser scanning microscopy. The fluorescence of DAPI, Alexa Fluor ${ }^{\circledR} 633$ phalloidin, and NPs are represented with blue (a, e), red $(\mathbf{b}, \mathbf{f})$, and yellow (c, g), respectively. Merged images of HuH-7 cells and MSCs from three channels are shown in (d) and (h), respectively. (i) and (j) are ortho-view images of z-stack, showing nanoparticles inside the cells. Scale bars: $10 \mu \mathrm{m}$ (HuH-7) and $20 \mu \mathrm{m}$ (MSC). For a, b, c, d, and i, pixel dwell time: $0.39 \mu \mathrm{s}$; frame size: 1024×1024; pixel size: $0.086 \mu \mathrm{m}$. For e, f, g, h, and j, pixel dwell time: $1.00 \mu \mathrm{s}$; frame size: $512 \times 512$; pixel size: $0.45 \mu \mathrm{m}$. (This figure is reprinted with copyright from[106])

The merged images from all three channels and the ortho-view of z-stack confirm that the NPs could be internalized by both HuH-7 tumor cells (Figure $8 \mathrm{a}-\mathrm{d}$, and i) and MSCs (Figure $8 \mathrm{e}-\mathrm{h}$, and j). Interestingly, the images in Figure 
8 (a-d, and i) suggest that the HuH-7 tumor cell nearly finished the mitotic (M) phase or cytokinesis when the mother cell split into two containing roughly equal shares of cellular components. As shown in Figure 8, the internalized NPs are mainly located in the perinuclear cytoplasm, while not exhibiting obvious affinity to any specific intracellular organelle. For further applications targeting at specific subcellular compartments, it is possible that the polymeric shell of these NPs could be functionalized with various ligands, such as nuclear localization signals (NLS) to facilitate NP passage through nuclear pore complex (NPC) for gene delivery[111], or mitochondrial localization signals to enhance specific drug delivery to mitochondria and improve the therapeutic efficacy.[112]

\subsubsection{Photostability of NPs in cellular imaging}

Photobleaching is always a major concern in bioimaging applications of organic fluorophores. To characterize the photostability of PCL-DPP-PCL NPs, we compared their performance with two popular commercial fluorophores, DAPI and Alexa Fluor ${ }^{\circledR} 633$ phalloidin, by a simultaneous photobleaching test for multiply-stained biological cells. After being stained with a cocktail of three dyes, MSCs were continuously exposed to 405, 561, and $633 \mathrm{~nm}$ laser irradiation and the fluorescence emission of each fluorophore was recorded at time points in 36.8-second intervals for a total duration of about $12 \mathrm{~min}$. A bandpass filter combination was applied with bandwidths tuned to record the emission in three channels: blue (421-481 nm), yellow (569-621 nm), and red $(650-758 \mathrm{~nm})$. Figure $9 \mathrm{a}$ show the merged images of the same multiply-stained MSC and the chronological decay of fluorescence intensity. It can be observed that all three fluorophores showed relatively high fluorescence intensity at the beginning of the test, while the subsequent photobleaching of three fluorophores were not synchronous. The fluorescence intensity of Alexa Fluor ${ }^{\circledR} 633$ phalloidin decayed most rapidly and the F-actin cytoskeleton was barely detectable after $110 \mathrm{~s}$. The fluorescence intensity of DAPI and NPs decreased much more slowly. Notably, the NPs were able to maintain strong fluorescence 
intensity during the test and maintain almost $90 \%$ of initial intensity even after $11 \mathrm{~min}$.

A quantitative analysis of the relative intensity change (Figure $9 \mathrm{~b}$ ) by curve fitting using exponential functions provided more insight of the decay kinetics of three different fluorophores. The PCL-DPP-PCL NPs exhibited monoexponential decay expressed by $\left[\mathrm{y}=\mathrm{A}_{1} * \exp \left(-\mathrm{t} / \tau_{1}\right)+\mathrm{y}_{0}\right]$, where $\left(\mathrm{A}_{1}+\mathrm{y}_{0}\right)$ represents the initial relative intensity, $\mathrm{y}_{0}$ is the relative residual fluorescence intensity, and $\tau_{1}$ is the decay rate constant called mean lifetime. The half-life was calculated as $\ln (2) * \tau_{1}$ (Table 1). DAPI also showed mono-exponential decay (Table 2) as reported in some previous studies[113, 114]. Although there was no prior study reporting the decay kinetics of Alexa Fluor ${ }^{\circledR} 633$ phalloidin, the fitting results showed that it could be described by a bi-exponential model $\left[y=A_{1} * \exp \left(-t / \tau_{1}\right)+A_{2} * \exp \left(-t / \tau_{2}\right)+y_{0}\right]$ (Table 3) in MSC. In this bi-exponential model, $\left(\mathrm{A}_{1}+\mathrm{A}_{2}+\mathrm{y}_{0}\right)$ represent the initial relative intensity; the half-life was computed based on the longer mean lifetime (Table 3). A comparison of the half-life values of three fluorophores in MSC (Tables 1-3) clearly showed that NPs (230 s) exhibited significantly higher photostability compared to DAPI (132 s) and Alexa Fluor ${ }^{\circledR} 633$ phalloidin (58.6 s).

As discussed previously, small-molecule DPP derivatives often show fluorescence quenching in solid states due to significant intermolecular $\pi-\pi$ aggregation. The reason why PCL-DPP-PCL NPs exhibit much greater photostability could be due to the covalent bonds between DPP and PCL chains, which efficiently suppressed the aggregation of DPP molecules. In order to verify this hypothesis, we prepared PCL/DPPHT nanoparticles (Figure 10 a-c) by simple physical blending (i.e. without any covalent bond formation between these two components $)$ of DPPHT $\left(8.0 \times 10^{-4} \mathrm{mmol}\right)$ and PCL $\left(M_{\mathrm{n}}=22,000\right.$, PDI $=1.3$ ) with a weight ratio similar to that of PCL-DPP-PCL. These physically blended PCL/DPP NPs could also be internalized by MSCs and accumulated in perinuclear region (Figure $11 \mathrm{a}-\mathrm{d}$, and i). However, photobleach test revealed 
that these physically blended NPs exhibited much weaker photostability (Figure 12) as compared to the covalently bonded PCL-DPP-PCL NPs (Figure 9). After $125 \mathrm{~s}$ of laser irradiation (561 nm), physically blended NPs could only retain about $13 \%$ of initial fluorescence intensity.

In addition to DPP-based fluorophores, this strategy to enhance the photostability could also be applied to other fluorophores with different excitation and emission wavelengths. As an example, we chose a quinacridone (QA) derivative (Figure 10g) as an initiator and synthesized PCL-QA-PCL NPs using a method similar to the preparation of PCL-DPP-PCL NPs. The SEM image (Figure 10d) showed that the resulting spherical particles had an average diameter of $117 \pm 21 \mathrm{~nm}$, which was consistent with the DLS (Figure 10f) and TEM (Figure 10e) results. Fluorescence microscopy (Figure 11e-h, and j) showed that they could be uptaken by MSCs and accumulated in the perinuclear region. The photobleach test suggested that the photostability of PCL-QA-PCL NPs (Figure 13) was relatively weaker than that of PCL-DPP-PCL NPs (Figure 9), which could be due to different properties of QA and DPP.

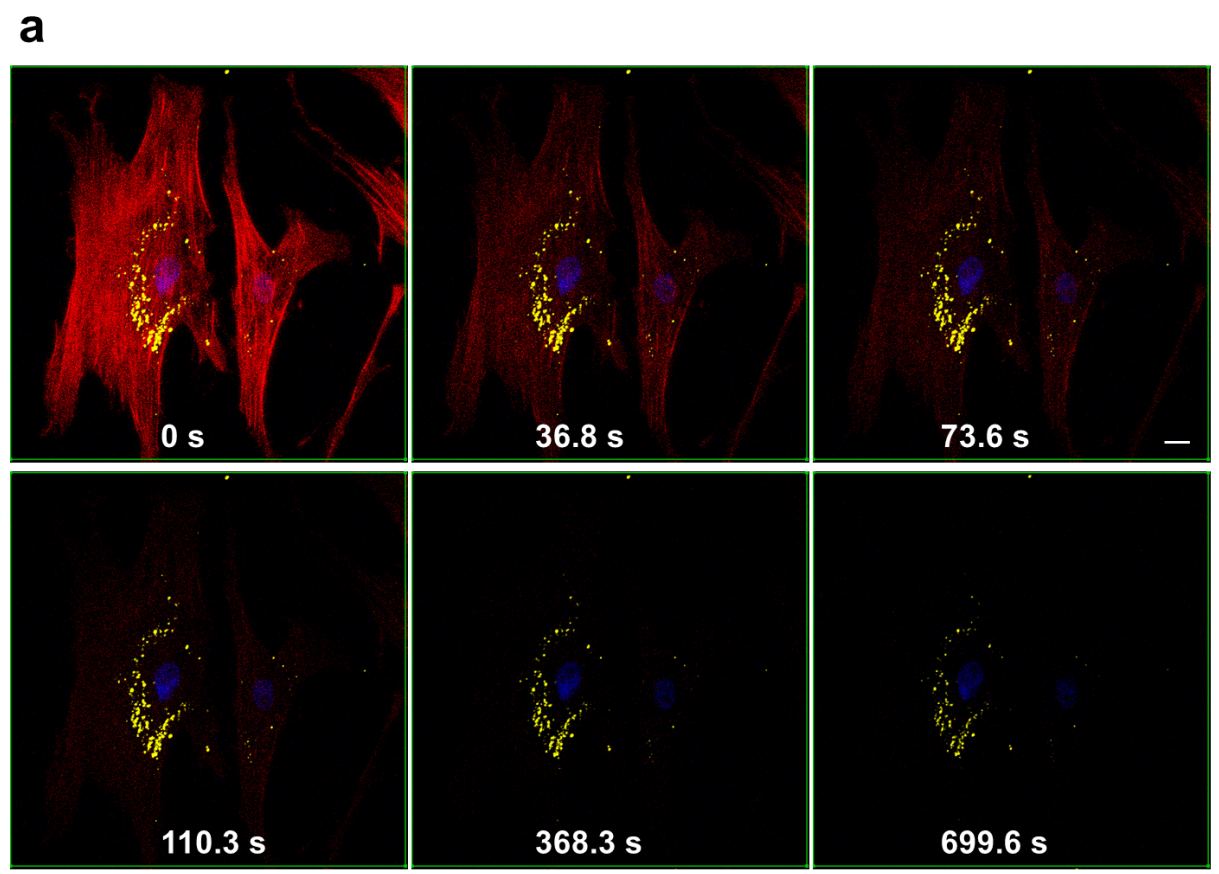




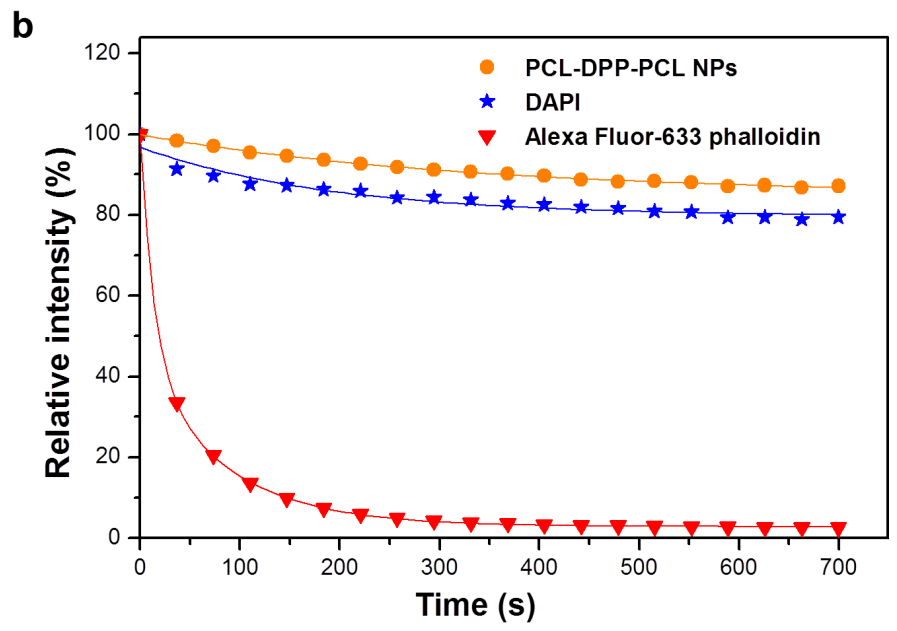

Figure 9. Photostability of fluorescent PCL-DPP-PCL nanoparticles (NPs) compared to Alexa Fluor® 633 phalloidin and DAPI in MSC. The fluorescences from DAPI, nanoparticles, and Alexa Fluor ${ }^{\circledR} 633$ phalloidin are represented by blue, yellow and red, respectively. (a) Chronological decay of fluorescence intensity of a multiply-stained MSC at different time points imaged by confocal laser scanning microscopy. The whole image field was photobleached. Pixel dwell time: $0.50 \mu$ s; frame size: $1024 \times 1024$; pixel size: $0.28 \mu \mathrm{m}$. Scale bar: $20 \mu \mathrm{m}$. (b) Relative intensity (instantaneous intensity / initial intensity) change of three fluorophores in the photobleached region. Curves are fitted using mono-exponential (PCL-DPPPCL NPs and DAPI) or bi-exponential functions (Alexa Fluor ${ }^{\circledR} 633$ phalloidin). The nominal powers of 405, 561, and $633 \mathrm{~nm}$ lasers are $20 \mathrm{~mW}, 20 \mathrm{~mW}$, and $5 \mathrm{~mW}$, respectively. (This figure is reprinted with copyright from[106])

Table 1. Parameters for fitting relative intensity change of PCL-DPP-PCL NPs in MSC (Figure 9b) using $\mathrm{y}=\mathrm{A}_{1} * \exp \left(-\mathrm{t} / \tau_{1}\right)+\mathrm{y}_{0}$ (Note: In this bi-exponential model, the half-life $\left(\mathrm{t} 1 / 2^{*}\right)$ was calculated by $\ln 2 * \tau 2$.) (This table is reprinted with copyright from[106])

\begin{tabular}{|c|c|c|}
\hline & Value & Standard Error \\
\hline $\mathbf{y}_{\mathbf{0}}$ & 84.99 & 0.32 \\
\hline $\mathbf{A}_{\mathbf{1}}$ & 14.97 & 0.28 \\
\hline Mean lifetime $\boldsymbol{\tau}_{\mathbf{1}}(\mathbf{s})$ & 331.82 & 17.28 \\
\hline Adj. $\mathbf{R}-\mathbf{s q u a r e}$ & 0.9967 & \\
\hline Half-life $\left(\mathbf{t}_{\mathbf{1} / \mathbf{2}}=\ln \mathbf{2} * \boldsymbol{\tau}_{\mathbf{1}}, \mathbf{s}\right)$ & 230.00 & \\
\hline
\end{tabular}


Table 2. Parameters for fitting relative intensity change of DAPI in MSC (Figure 9b) using $y=$ $\mathrm{A}_{1} * \exp \left(-\mathrm{t} / \tau_{1}\right)+\mathrm{y}_{0}$ (Note: In this bi-exponential model, the half-life ( $\left.\mathrm{t} 1 / 2 *\right)$ was calculated by $\ln 2 * \tau 2$.) (This table is reprinted with copyright from[106])

\begin{tabular}{|c|c|c|}
\hline & Value & Standard Error \\
\hline $\mathbf{y}_{\mathbf{0}}$ & 79.64 & 0.80 \\
\hline $\mathbf{A}_{\mathbf{1}}$ & 17.17 & 1.09 \\
\hline Mean lifetime $\boldsymbol{\tau}_{\mathbf{1}} \mathbf{( s )}$ & 190.62 & 32.56 \\
\hline Adj. R-square & 0.9288 & \\
\hline Half-life $\left(\mathbf{t}_{\mathbf{1} / \mathbf{2}}{ }^{*}=\ln \mathbf{2}^{*} \boldsymbol{\tau}_{\mathbf{1}} \mathbf{,} \mathbf{s}\right)$ & 132.13 & \\
\hline
\end{tabular}

Table 3. Parameters for fitting relative intensity change of Alex Fluor ${ }^{\circledR} 633$ in MSC (Figure 9b) using $\mathrm{y}=\mathrm{A}_{1} * \exp \left(-\mathrm{t} / \tau_{1}\right)+\mathrm{A}_{2} * \exp \left(-\mathrm{t} / \tau_{2}\right)+\mathrm{y}_{0} \quad$ (Note: In this bi-exponential model, the half-life $(\mathrm{t} 1 / 2 *)$ was calculated by $\ln 2 * \tau 2$.) (This table is reprinted with copyright from[106])

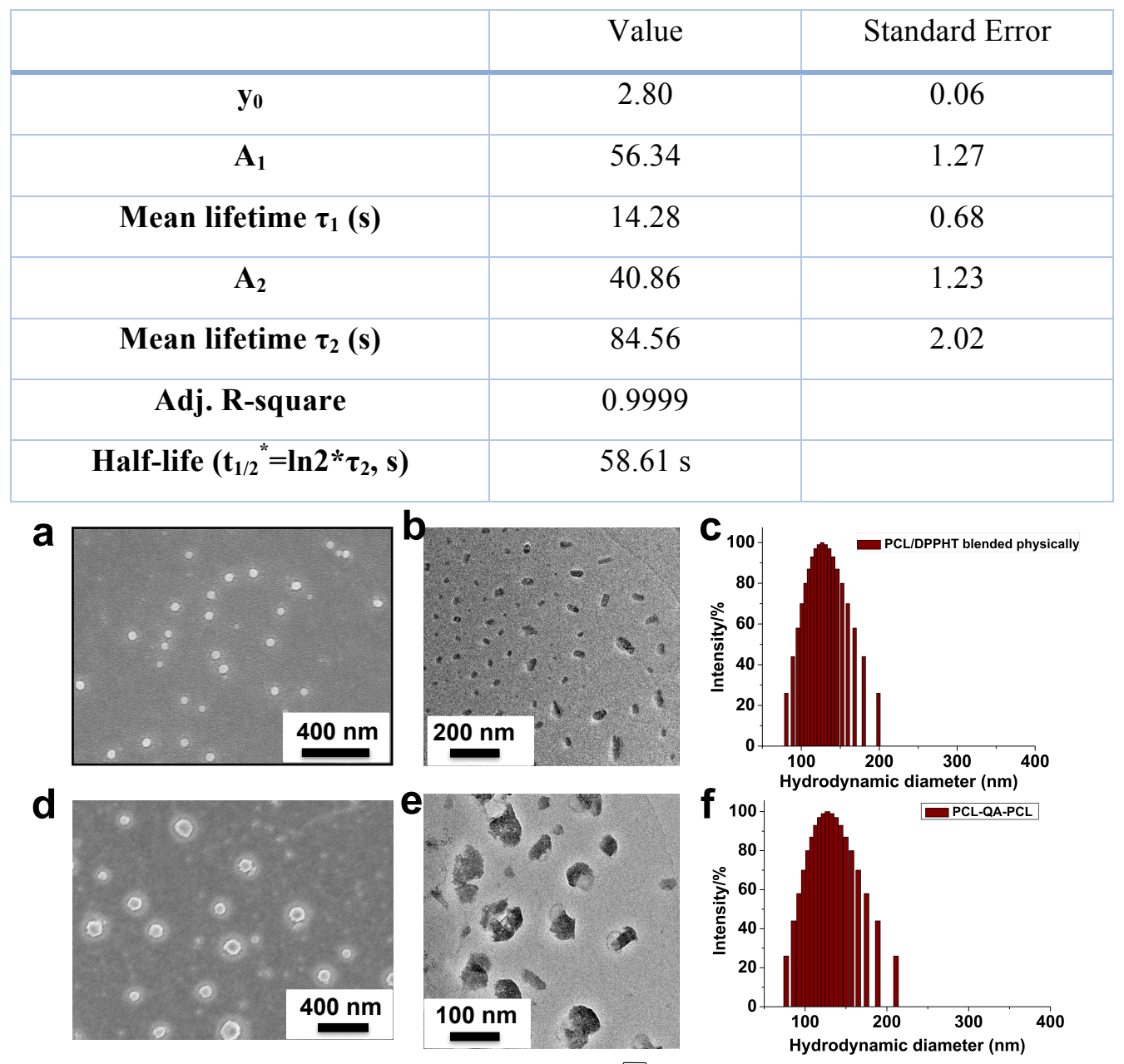




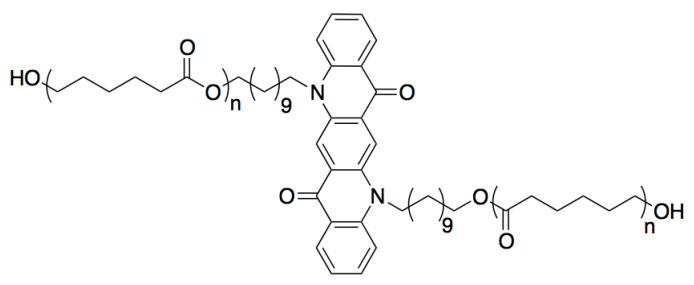

Figure 10. SEM (a, d), TEM (b, e) and DLS (c, f) characterization of physically blended DPPHT/PCL NPs (a-c) and PCL-QA-PCL NPs (d-f). (g) The chemical structure of PCL-QAPCL. (This figure is reprinted with copyright from[106])

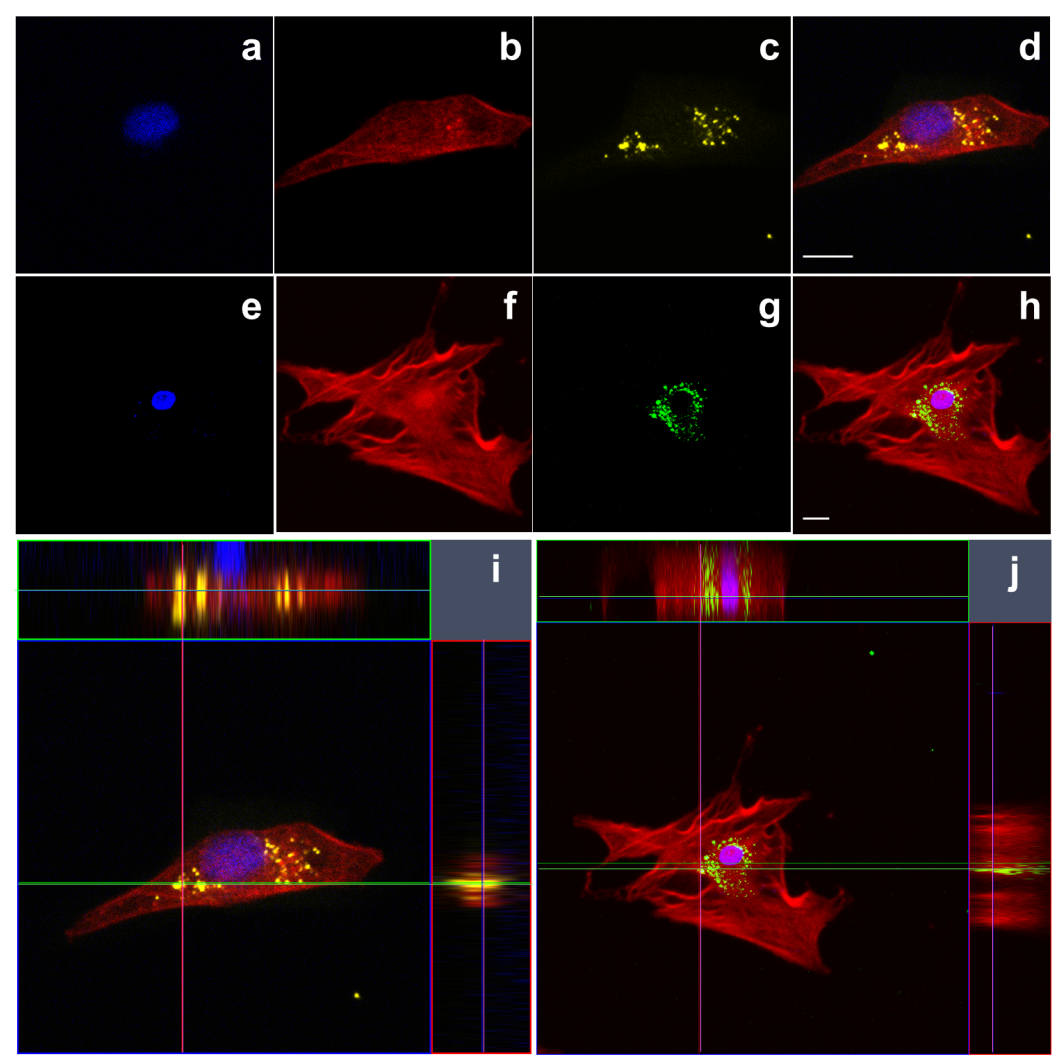

Figure 11. Cellular uptake of physically blended PCL/DPPHT NPs (a-d and i) and PCL-QAPCL nanoparticles (e-h and j) in MSCs imaged by confocal laser scanning microscopy. The fluorescence of DAPI, Alexa Fluor ${ }^{\circledR} 633$ phalloidin, physically blended PCL/DPPHT NPs, and PCL-QA-PCL nanoparticles are pseudo-labeled with blue (a, e), red (b, f), yellow (c), and green (g), respectively. Merged images of MSCs from different channels are shown in (d) and (h), respectively. (i) and (j) are ortho-view images of z-stack, showing they could be uptaken by the cells. Scale bars: $20 \mu \mathrm{m}$. (For a-d and i, pixel dwell: $1.27 \mu$ s, frame size: $512 \times 512$; for e-h, and $\mathrm{j}$, pixel dwell: $0.63 \mu \mathrm{s}$, frame size: $1040 \times 1040$ ). (This figure is reprinted with copyright from[106]) 


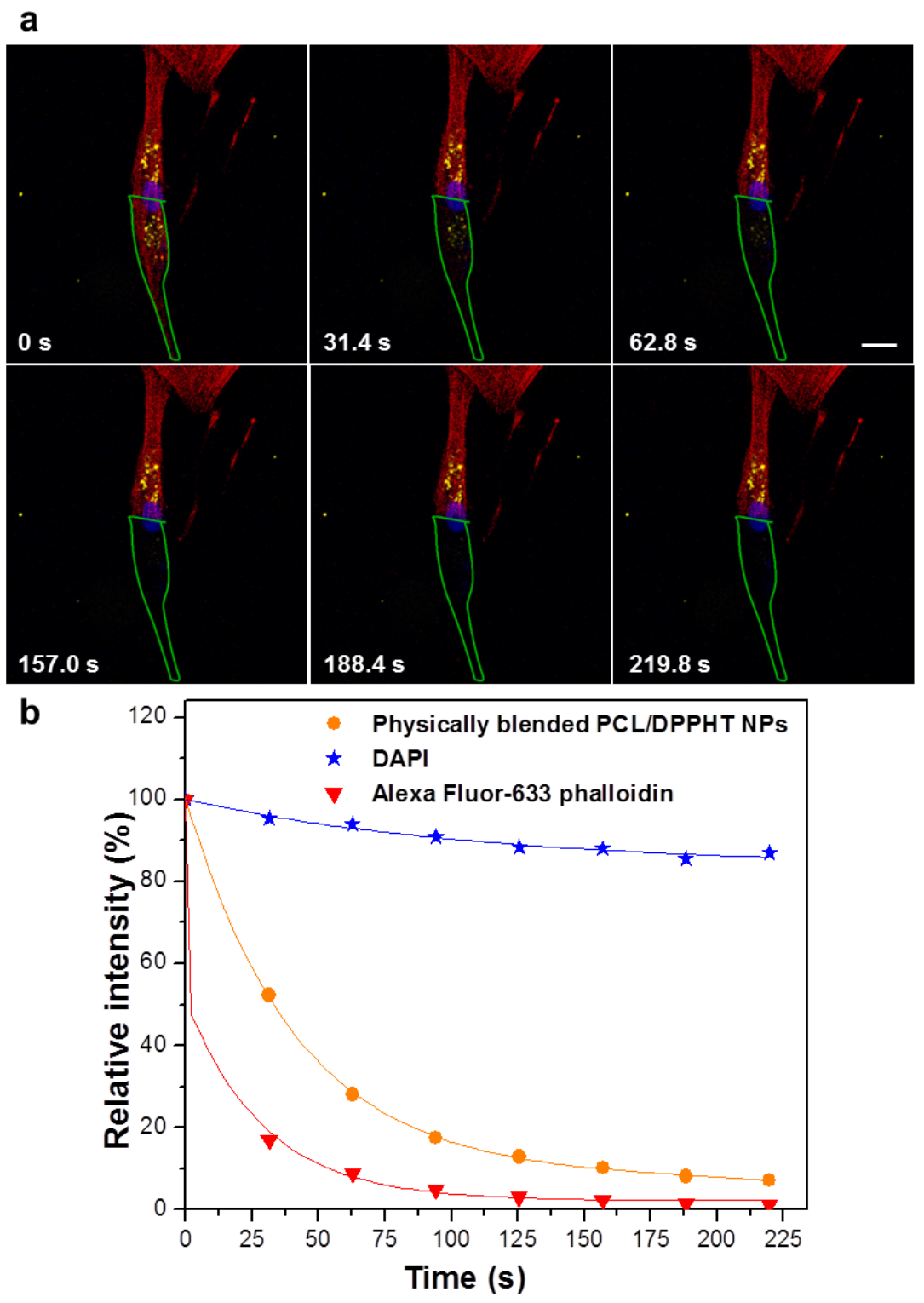

Figure 12. Photostability of physically blended PCL/DPPHT NPs compared to Alexa Fluor® 633 phalloidin and DAPI in a MSC. The fluorescence of DAPI, physically blended PCL/DPPHT NPs, and Alexa Fluor ${ }^{\circledR} 633$ phalloidin are represented with blue, yellow and red, respectively. (a) Chronological decay of fluorescence intensity of a multiply-stained MSC at different time points imaged by confocal laser scanning microscopy. The green polygon indicates the region photobleached. Pixel dwell time: $1.27 \mu$ s; frame size: $512 \times 542$; pixel size: $0.37 \mu \mathrm{m}$. Scale bar: $20 \mu \mathrm{m}$. (b) Relative intensity (instantaneous intensity / initial intensity) change of three fluorophores in the photobleached region. Curves are fitted using mono-exponential (DAPI) or bi-exponential functions (physically blended PCL/DPPHT nanoparticles and Alexa Fluor ${ }^{\circledR} 633$ phalloidin). (This figure is reprinted with copyright from[106]) 


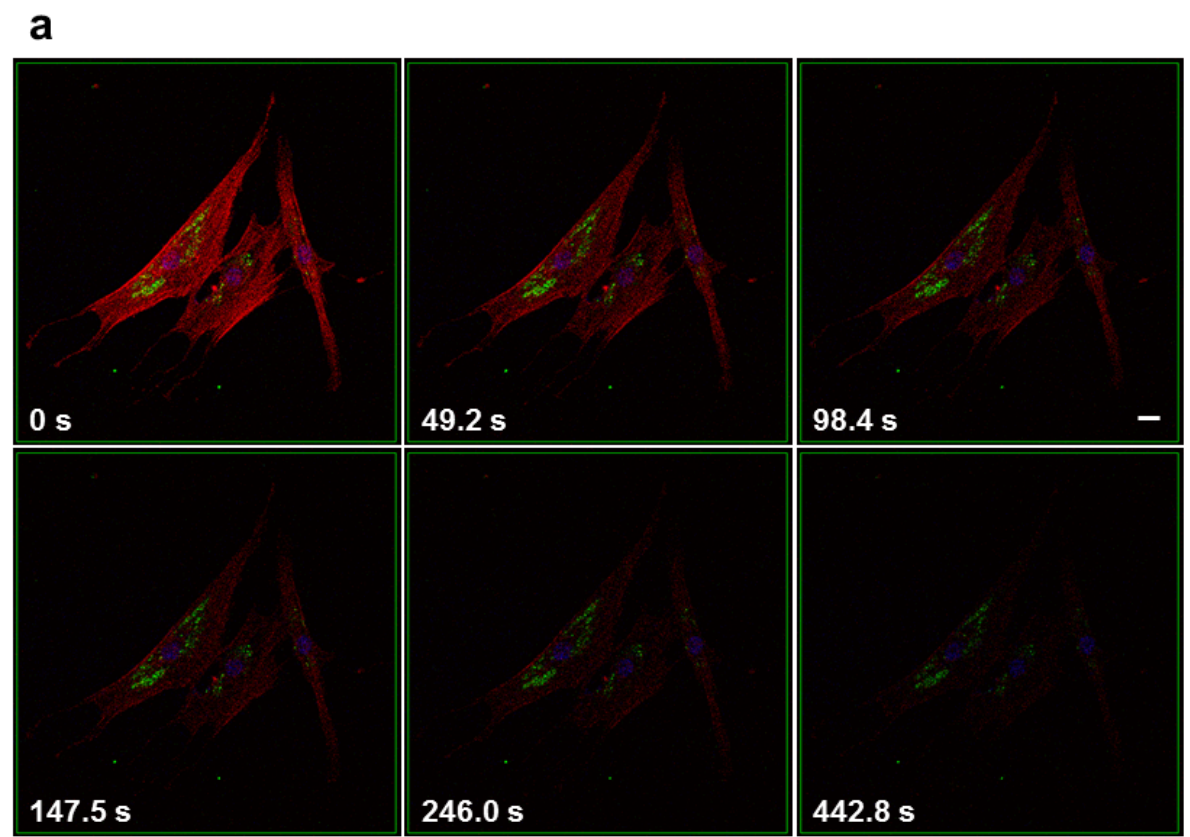

b

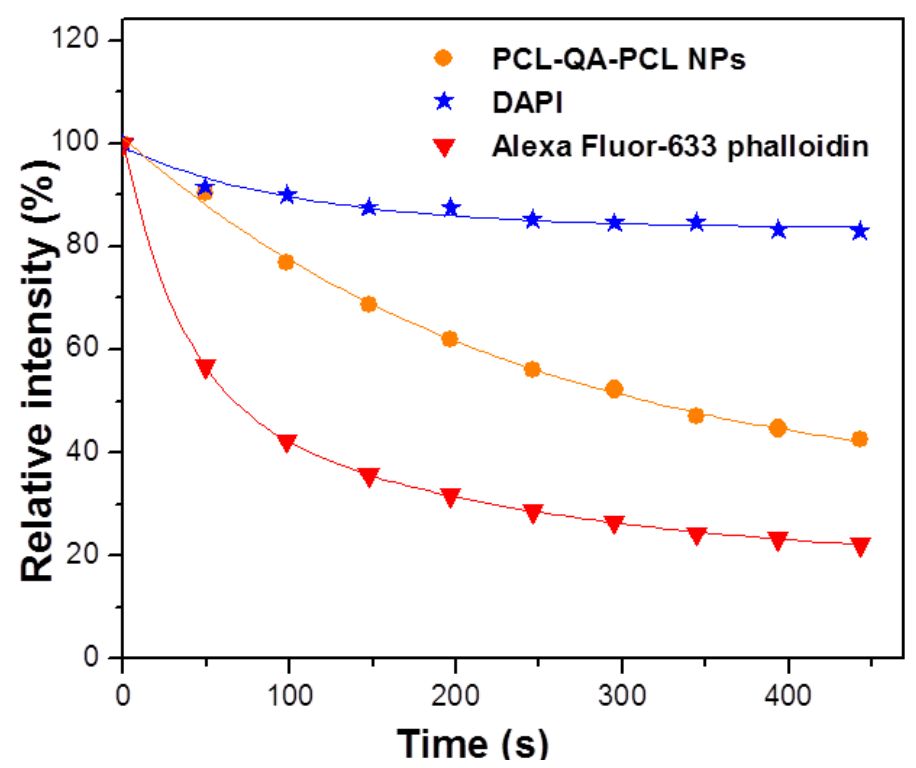

Figure 13. Photostability of PCL-QA-PCL NPs compared to Alexa Fluor ${ }^{\circledR} 633$ phalloidin and DAPI in a MSC. The fluorescences from DAPI, PCL-QA-PCL nanoparticles, and Alexa Fluor ${ }^{\circledR}$ 633 phalloidin are represented with blue, green, and red, respectively. (a) Chronological decay of fluorescence intensity of a multiply-stained MSC at different time points imaged by confocal laser scanning microscopy. The whole image field was photobleached. Pixel dwell time: 0.64 $\mu$ s; frame size: $1024 \times 1024$; pixel size: $0.42 \mu \mathrm{m}$. Scale bar: $20 \mu \mathrm{m}$. (b) Relative intensity (instantaneous intensity / initial intensity) change of three fluorophores in the photobleached region. Curves are fitted using mono-exponential (DAPI) or bi-exponential functions (PCL-QAPCL NPs and Alexa Fluor ${ }^{\circledR} 633$ phalloidin). (This figure is reprinted with copyright from[106]) 


\subsubsection{Long-term cell tracing using PCL-DPP-PCL NPs vs. Qtracker ${ }^{\circledR}$}

In addition to the photostability of fluorophore upon exposure to light, prior studies have suggested that the decrease of fluorescence intensity of stained cells in long-term study could be also due to loss of fluorophores during cell division and exocytosis[115]. Therefore we investigated the application of PCLDPP-PCL NPs for long-term cell tracing by fluorescence imaging, and compared their performance with a commercial counterpart, the Qtracker ${ }^{\circledR}$ cell labeling kit consisting of highly fluorescent quantum dot nanocrystals (nanoscale atom clusters comprising a core, a shell and surface coating).

HuH-7 tumor cells were firstly incubated with NPs and Qtracker ${ }^{\circledR}$ for $48 \mathrm{~h}$ at concentration of $2 \mathrm{nM}$, respectively. Then the excess fluorophores were removed and washed by $1 \times \mathrm{PBS}$. The cells were then subcultured into a 6 -well plate and cultured for up to 7 days. The fluorescence change of the labeled cells was monitored by fluorescence microscopy (Figure 15) and quantitatively analyzed at specific time points by flow cytometry for about 10,000 cell events in each test (Figure 14). The fluorescence of $\mathrm{HuH}-7$ cells labeled with either NPs or Qtracker ${ }^{\circledR}$ gradually decayed and shifted from the high intensity spectrum (Day 1) toward the weak intensity regime (Day 7). It was noted that the NP-labeled tumor cells exhibited much higher fluorescence stability with $49.9 \%$ of the population remaining to be strongly fluorescent at Day 7; whereas only $8.68 \%$ of the Qtracker ${ }^{\circledR}$-labeled cells maintained their fluorescence at Day 7. Similar comparative study was also performed with MSC culture for up to 14 days (Figure 16). The flow cytometry analysis and fluorescence microscopy again demonstrated the impressive long-term photostability of this PCL-DPPPCL NP system, with $46.7 \%$ of the NP-stained MSCs retaining strong fluorescence after 2 weeks. These results indicate that the PCL-DPP-PCL NPs are highly advantageous as a stable fluorophore particularly for applications related to long-term cell tracing. 


\subsubsection{Cytotoxicity of PCL-DPP-PCL NPs.}

Finally, we evaluated the cytotoxicity of the PCL-DPP-PCL NPs using PrestoBlue assay. PrestoBlue ${ }^{\circledR}$ reagent, Resazurin $\left(\lambda_{\text {max.abs }}=600 \mathrm{~nm}\right)$ is a nonfluorescent blue compound, can be reduced in live cells to red in color and highly fluorescent resorufin $\left(\lambda_{\text {max.abs }}=571 \mathrm{~nm}\right)$. Since the number of metabolically active cells is proportionally related with the reduction level, the data from absorbance readings can be represented as the percentage reduction of the PrestoBlue reagent, indicating the relative cell viability.

HuH-7 tumor cells and MSCs were incubated with NPs of different concentrations (up to $4 \mathrm{mg} / \mathrm{mL}$ ) for $24 \mathrm{~h}$ and $72 \mathrm{~h}$. Percentage reduction in each sample was measured with regard to that in cell culture without NP staining (the control).

Figure 17 shows that the cell viability of MSCs and HuH-7 cells was not significantly affected for up to $72 \mathrm{~h}$, indicating minimum cytotoxicity of the PCL-DPP-PCL NPs. Statistical analysis by paired student t-test indicated that there was no significant difference between groups treated with different concentrations of NPs and the control for both HuH-7 and MSCs ( $p>0.5)$.
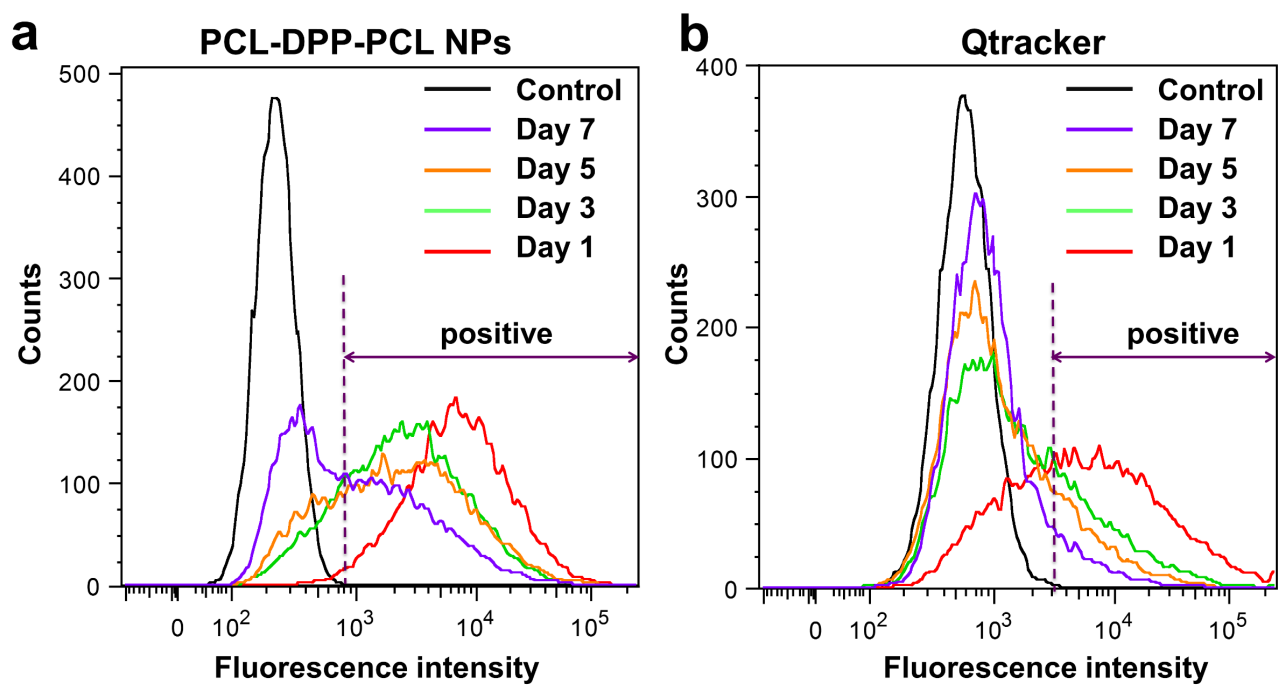


\section{PCL-DPP-PCL NPs}
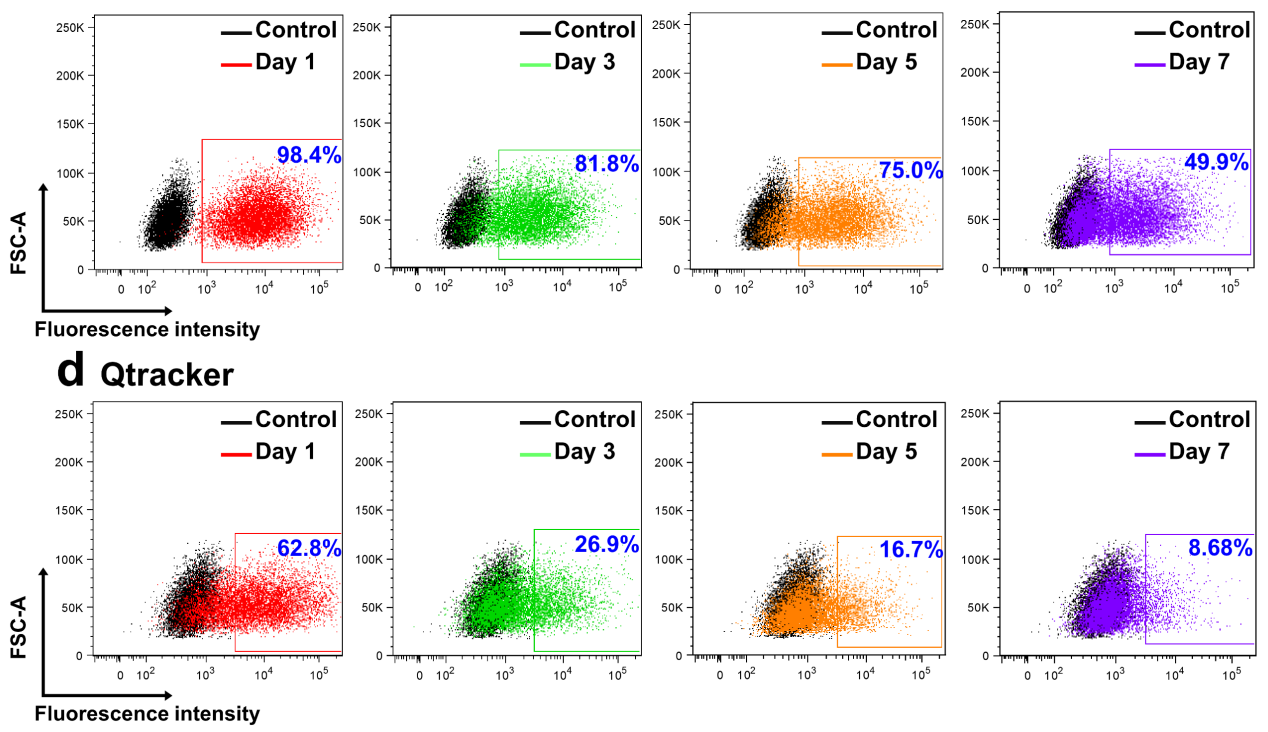

Figure 14. Long-term tracing of HuH-7 tumor cells using (a, c) PCL-DPP-PCL nanoparticles and (b, d) Qtracker ${ }^{\circledR}$. The fluorescence intensity change of the labeled cells was measured by flow cytometry analyses at different time points. The control represents the unlabeled cells. The percentages of cells with positive signals in $(\mathrm{c}, \mathrm{d})$ were identified based on the gating thresholds shown in $(a, b)$ for NP and Qtracker ${ }^{\circledR}$, respectively. (This figure is reprinted with copyright from[106])

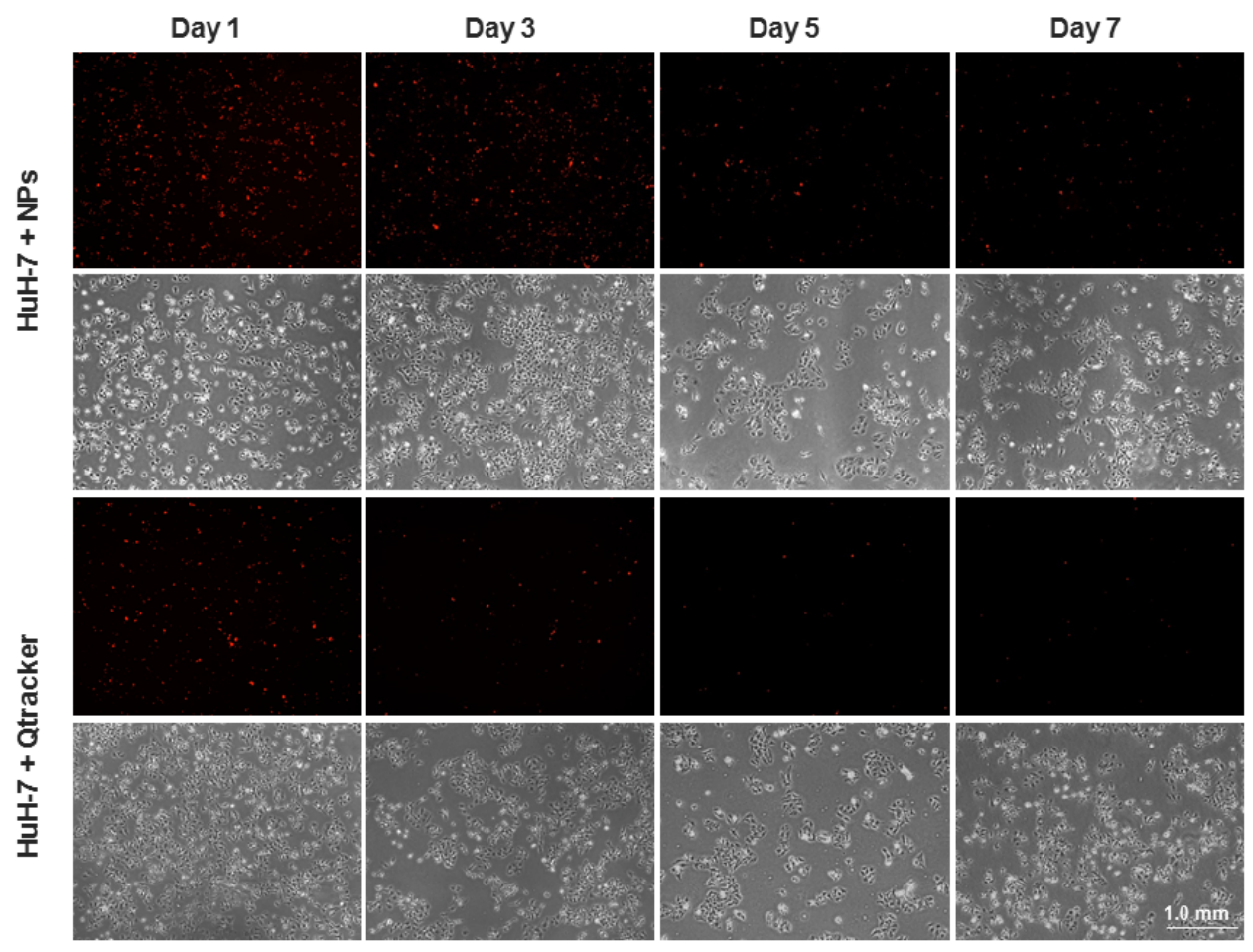


Figure 15. Long-term tracing of HuH-7 tumor cells using PCL-DPP-PCL NPs and Qtracker ${ }^{\circledR}$. The fluorescence intensity change of the labeled cells is characterized by optical contrast and fluorescence microscopy measured at different time points. (This figure is reprinted with copyright from[106])

a

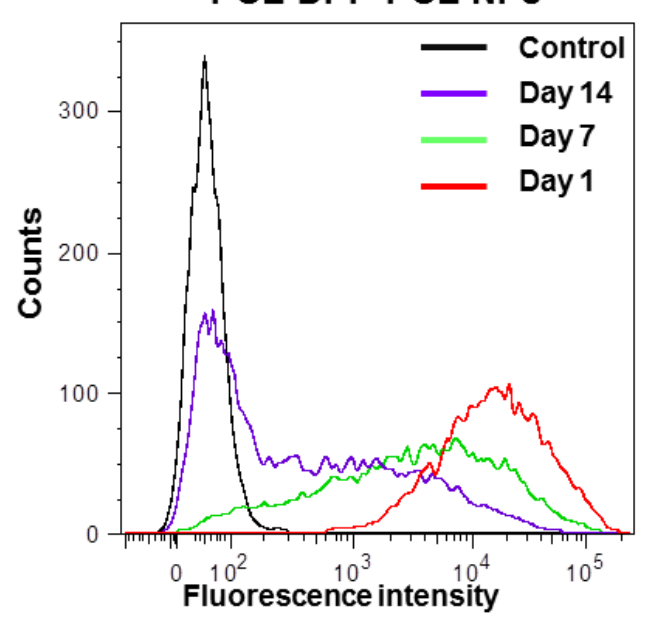

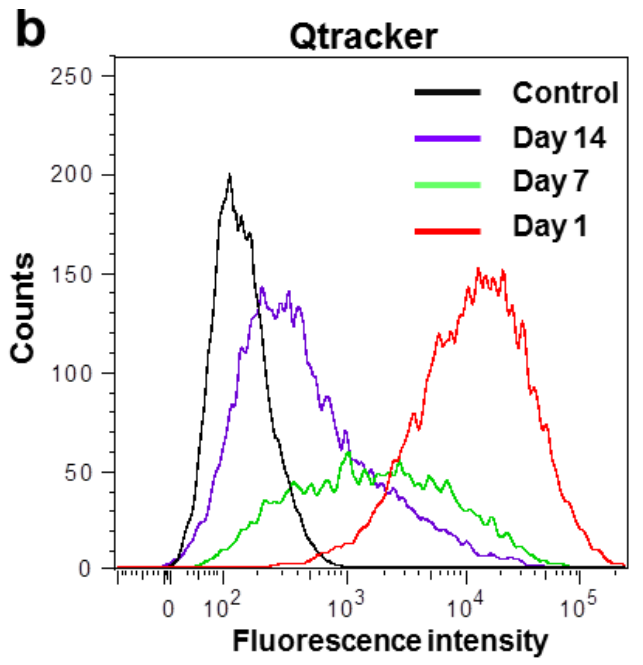

\section{PCL-DPP-PCL NPs}
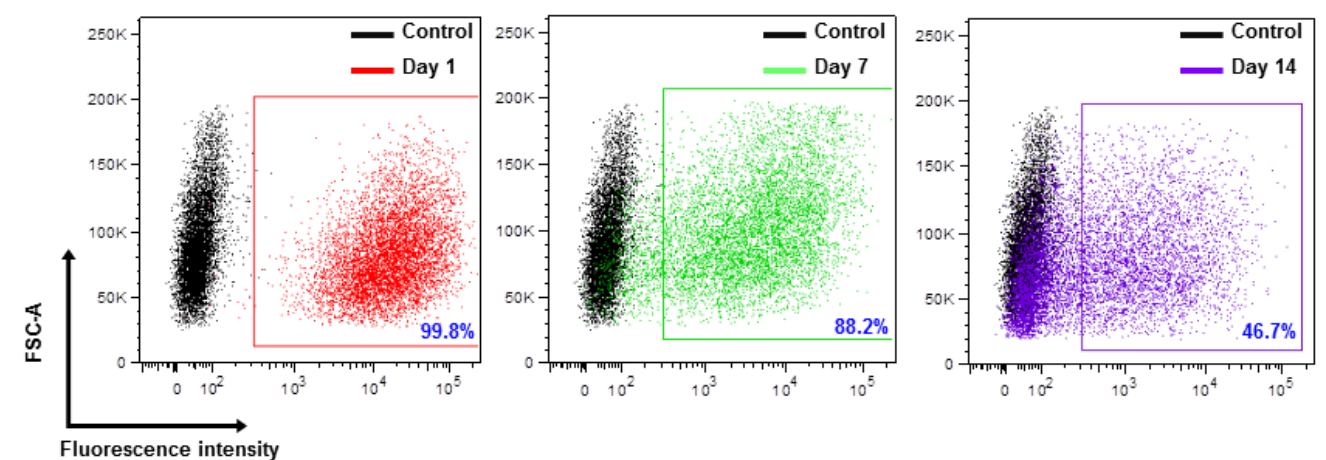

d Qtracker
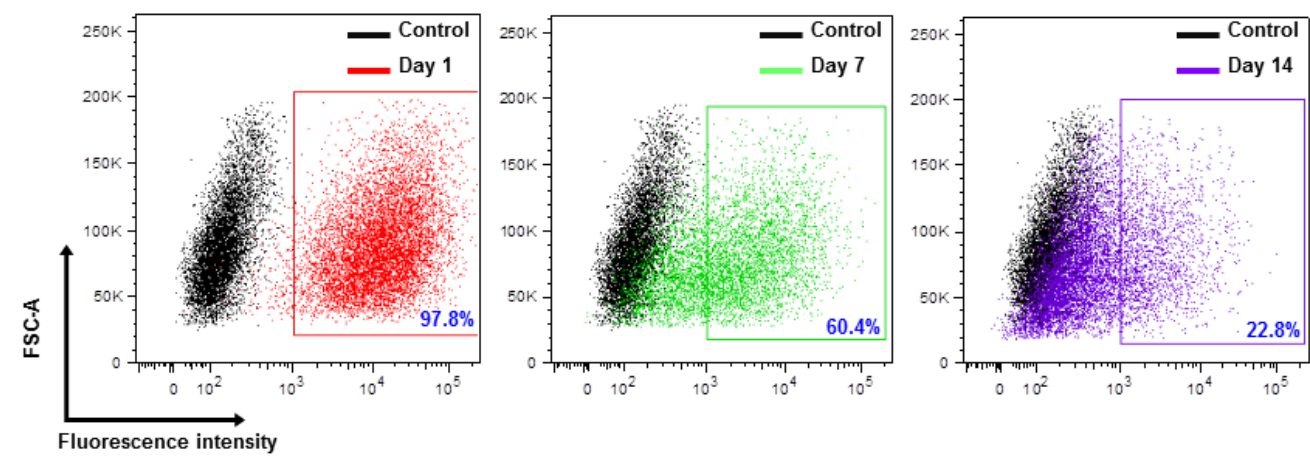
e
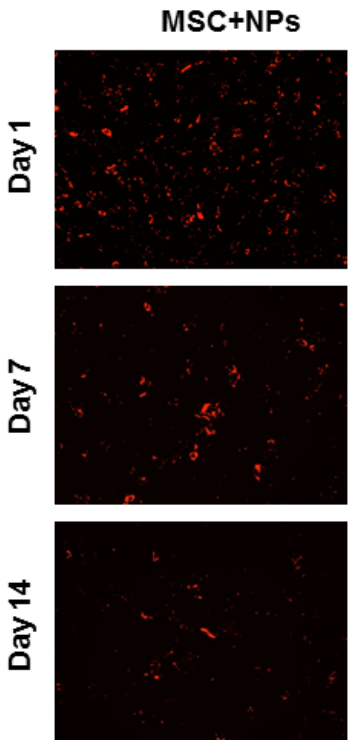
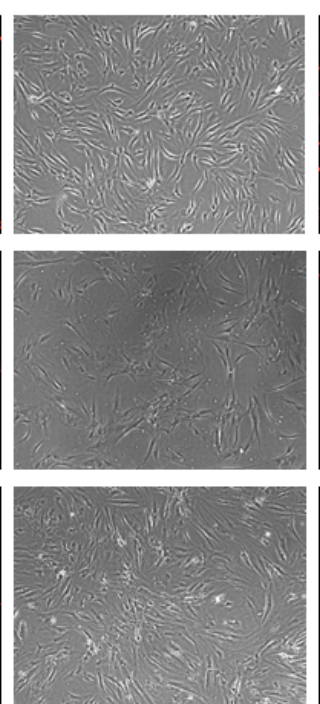

MSC+Qtracker
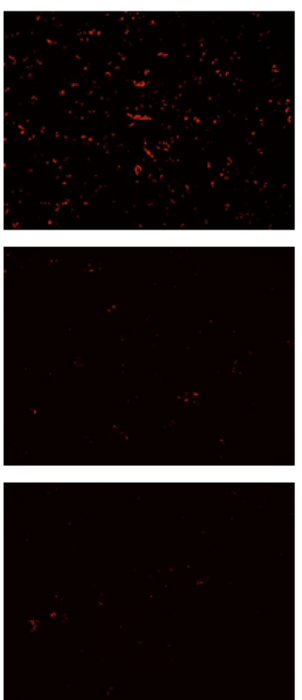
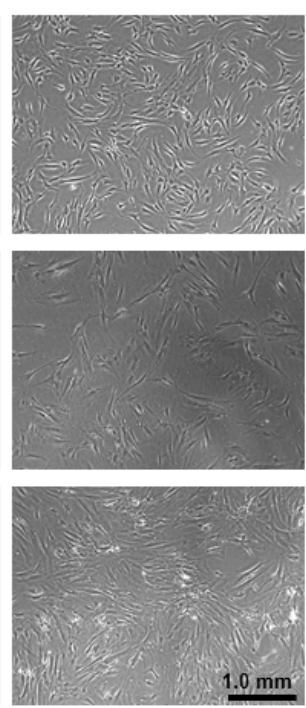

Figure 16. Long-term tracing of MSCs using PCL-DPP-PCL NPs and Qtracker ${ }^{\circledR}$. The fluorescence intensity change of the labeled cells is characterized by (a) flow cytometry histograms with PCL-DPP-PCL NPs, (b) flow cytometry histograms with Qtracker, (c) flow cytometry dot plots with PCL-DPP-PCL NPs, (d) flow cytometry dot plots with Qtracker, (e) optical contrast and fluorescence microscopy measured at different time points. (This figure is reprinted with copyright from[106])

a

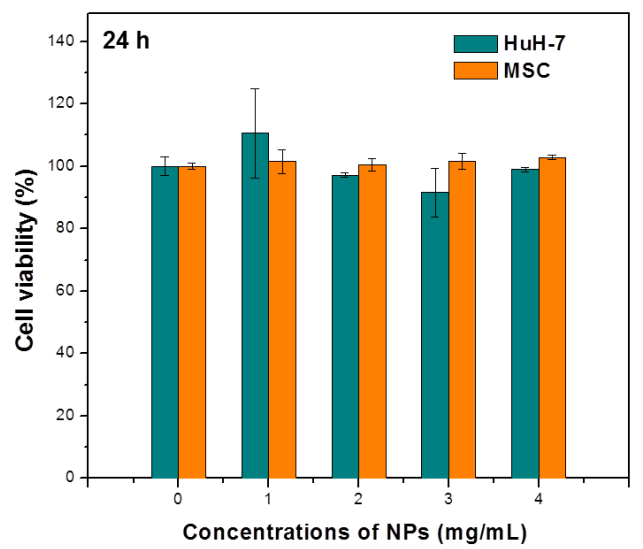

b

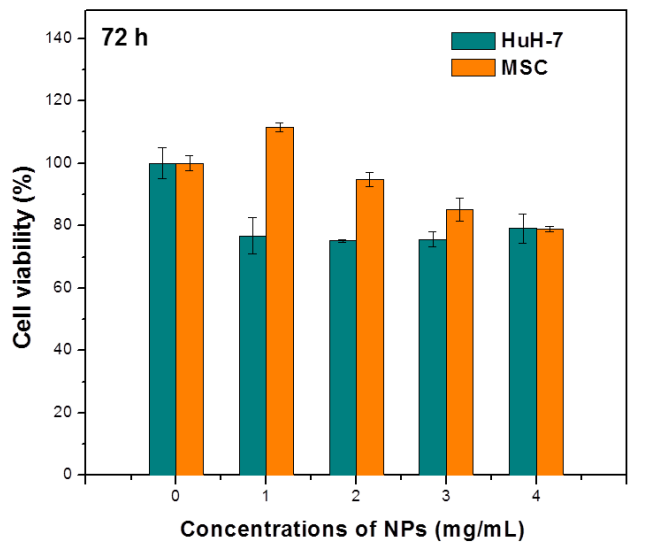

Figure 17. Cell viabilities of HuH-7 and MSCs after incubating with different concentrations of PCL-DPP-PCL NPs for (a) $24 \mathrm{~h}$ and (b) $72 \mathrm{~h}$ tested by PrestoBlue assay. Blank cells without any nanoparticles treatment were regarded as control. Values were expressed as means $\pm \mathrm{SD}$ $(n=3)$. Paired student $t$-test was used for statistical analysis between groups treated with different concentrations of NPs and control ( $p>0.5$ ). (This figure is reprinted with copyright from[106]) 


\subsection{Conclusions}

In summary, we have presented a new type of highly fluorescent and bioresorbable polymeric NPs prepared through nanoprecipitation of a welldefined polymer, PCL-DPP-PCL, in the presence of Pluronic ${ }^{\circledR} 127$ as the stabilizer. A key feature of our molecular design lies in the covalent insertion of DPP as a highly fluorescent moiety into the middle of each PCL chain. Such covalent bonding at the interface of DPP and PCL as well as the steric effect endowed by long PCL chains effectively suppress the $\pi-\pi$ aggregation of DPP in the PCL matrix. As a consequence, PCL-DPP-PCL with $M_{\mathrm{n}}$ of 38,300 Da showed bright fluorescence in solid states. The NPs prepared through a nanoprecipitation process were highly fluorescent and could be internalized by both HuH-7 tumor cells and MSCs with little cytotoxicity to these cells. Most importantly, the PCL-DPP-PCL NPs exhibited significantly enhanced photostability compared to several commercial organic fluorophores, the physically blended DPPHT/PCL NPs and Qtracker ${ }^{\circledR}$ for imaging of both tumor cells and stem cells. These unique properties make the PCL-DPP-PCL NPs promising imaging probes to track a broad range of biological systems. Compared to previously reported fluorescent organic or polymeric NPs[39, 94], the $1.3 \%$ weight fraction of fluorescent components in these NPs is significantly lower and the polymer matrix (i.e. PCL) has proved to be biocompatible and bioresorbable. Therefore, the potential toxicity, if any, caused by the encapsulated fluorophores is minimized. Moreover, the fluorescence excitation and emission wavelengths of these synthetic NPs can be customized and finely tuned during synthesis for specific applications. Currently we are extending this strategy to polymers with strong fluorescence in near infrared range that is important for in-vivo imaging.

(Republished with permission of [Royal Society of Chemistry] from[106]); permission conveyed through Copyright Clearance Center, Inc.) 


\section{Chapter 3. Long-term Tracking Mesenchymal Stem Cell Differentiation with Photostable Fluorescent Nanoparticles}

\subsection{Introduction}

Stem cell-based therapies, aiming to treat diseases using the self-renewal and multilineage differentiation capacities of the transplanted stem cells, have achieved great progress in the past decade [116-119]. Among the various types of stem cells, mesenchymal stem cells (MSCs) are much less prone to the ethical issues and the risk of developing teratoma [120]. MSCs are able to differentiate into multiple types of mesoderm lineages, such as chondrocytes, osteocytes, adipocytes and some ectodermic and endodermic cells [120]. An increasing number of MSC-based therapies, especially for tissue repair or immune disorder diseases, have been attempted since 2004 [121]. However, there are many major issues remaining to be resolved before successful clinical applications on real patients $[120,122]$. One of the critical challenges is the difficulty to track the fate, distribution and migration of the transplanted stem cells in the local microenvironment due to the limited tracking methods. Compared to traditional but highly invasive histological analysis, non-invasive imaging techniques are urgently demanded to track the transplanted stem cells for monitoring the therapeutic progress. The distribution and migration of the transplanted stem cells can provide a reliable evaluation for the cell-based therapeutic effect and elucidate the optimal conditions during surgery, such as cell dosage, route of delivery and the timing of transplantation [123, 124]. Additionally, in the applications of stem cell based tissue and organ regeneration, it is essential to delineate the specific contributions of transplanted cells and host cells to the damaged tissues or organs [125].

Fluorescence imaging has been widely applied in various biological fields as a non-invasive technique because of its unique advantages, such as high 
sensitivity, rapid acquisition, lack of radiation, low cost and easy access [124129]. The most popular labelling markers for fluorescence imaging are organic dyes, such as DAPI (4',6-diamidino-2-phenylindole) and Alexa Fluor family fluorophores. However, most organic dyes can only be used for short-term imaging because of photobleaching effect [125]. Another emerging type of fluorescent labels are quantum dots (QD), which emit strong fluorescence due to quantum confinement effect and the excitation and emission spectra are highly tunable based on their unique sizes $[125,130]$. However, the typical semiconductor and heavy metal-based quantum dots could release metal ions particularly under UV illumination, which are highly toxic to the cells both in vitro and in vivo [131-133]. QDs comprising a cadmium selenide (CdSe) nanocrystal core and a zinc sulfide $(\mathrm{ZnS})$ shell were developed to label human MSCs during osteogenic differentiation by liposome-mediated transportation [134]. It was found that although the labelled MSCs showed normal proliferation, the gene expressions of specific osteogenic markers including osteopontin, osteocalcin and alkaline phosphatase were significantly inhibited in the presence of QDs [134]. In another prior study, MSCs labelled with commercial quantum dots (QTracker 800, Thermo Fisher Scientific) were delivered to nude rats with segmental bone defects to track the cell distributions during the bone healing process after transplantation [131]. Although clear fluorescence could be observed in vivo from the treated rats, the QD-labelling reduced the function of transplanted cell and thereby the bone healing responses. To address this major issue, QD probes need to be modified with biomolecules or biocompatible polymers to reduce their cytotoxicity before applications. For example, CGGGRGD (Cys-Gly-Gly-Gly-Arg-Gly-Asp) was covalently bonded with $\mathrm{CdSe} / \mathrm{ZnS}$ QDs to label human MSCs for induced differentiations into adipogenic, chondrogenic, and osteogenic lineages [125]. These bioconjugated QDs were shown to be able to maintain the viability as well as the multipotency of the labelled human MSCs (hMSCs). In addition to quantum dots, other nanoprobes, such as phosphonate-functionalised 
polystyrene nanoparticles were also used for long-term tracing of hMSC differentiation after they were labelled with the fluorescent dye N-(2,6diisopropylphenyl)-perylene-3,4-dicarboximide [135].

Previously, Huang and co-workers have prepared a well-defined synthetic polymer platform based on di(thiophene-2-yl)-diketopyrrolopyrrole (DPP) covalently linked in the middle of biocompatible and bioresorbable polycaprolactone (PCL) polymer, which is abbreviated as PCL-DPP-PCL [106]. These nanoparticles exhibited bright fluorescence and strong photostability for cancer and stem cell imaging. Although the basic material properties, photostability and cytotoxicity to a few tumor cells and stem cells have been elaborated in details [106], however the potential influence of this polymeric nanoprobe on MSC multipotency and differentiation remains unclear. Herein we bring this study from cellular level to tissue level by longterm tracing MSCs labelled with PCL-DPP-PCL and elucidating the potential effect of these nanoparticles on the MSC multipotency. Specifically, we labelled hMSCs with PCL-DPP-PCL nanoparticles and then induced these labelled hMSCs into different cell lineages including adipocytes, chondrocytes, and osteocytes respectively, which were characterized by gene expression and histological staining. Additionally, the residue fluorescence within the differentiated labelled cells were measured by flow cytometry and confocal laser scanning microscopy. We believe that this work could be interesting and inspiring to researchers in the thriving fields of stem cell based therapies, tissue engineering and regenerative medicine.

\subsection{Materials and Method}

\subsubsection{Preparation of PCL-DPP-PCL NPs}

The detailed procedure to prepare and characterize the PCL-DPP-PCL nanoparticles (NPs) can be found in the previous report [106]. The prepared NPs were dispersed in sterile $1 \times$ PBS before use. 


\subsubsection{Cell culture}

Human bone marrow derived mesenchymal stem cells (hMSCs) were obtained from consented patients in the National University Hospital (Singapore) following a protocol approved by Institution Review Board (IRB) [136]. The isolated cells were expanded in expansion medium, consisting of low glucose Dulbecoo's Modified Eagle Medium (DMEM) supplemented with sodium pyruvate and GlutaMax ${ }^{\mathrm{TM}}$ (Life Technologies, Singapore), 10\% Fetal Bovine Serum (FBS) (Life Technologies, Singapore) and 100 units/ml penicillinstreptomycin (Life Technologies, Singapore), and incubated under $37^{\circ} \mathrm{C}$ with $5 \% \mathrm{CO}_{2}$ and humidified atmosphere. Non-adherent cells were removed after 48 h. The adherent cells remained in culture flasks for further expansion. The hMSCs in passages 3 and 4 were used for this study.

\subsubsection{Cell labeling and flow cytometry}

The hMSCs were seeded in 6-well plates in normal DMEM medium with 10\% FBS and 1\% penicillin-streptomycin. After cell attachment overnight, the original medium was replaced by fresh medium with dispersed PCL-DPP-PCL NPs at concentration of $0.2 \mathrm{mg} / \mathrm{mL}$. Then the cells were grown at $37^{\circ} \mathrm{C}$ with $5 \%$ $\mathrm{CO}_{2}$ for 3 and 7 days before the excess NPs were rinsed with $1 \times$ PBS.

For confocal laser scanning microscopy, the cells were fixed by formalin and then permeabilized by $0.1 \%$ Triton-X. Then the samples were blocked by $1 \%$ BSA and stained with Alexa Fluor ${ }^{\circledR} 633$ phalloidin for F-actin and DAPI for nucleus. The 3-color laser source $(405 \mathrm{~nm}, 561 \mathrm{~nm}$ and $633 \mathrm{~nm})$ in a confocal microscope (Carl Zeiss, LSM 710) provided excitation for the fluorophores in DAPI, PCL-DPP-PCL and Alexa Fluor ${ }^{\circledR} 633$ phalloidin with corresponding emission windows at 410-549 nm, 566-622 nm and 638-747 nm, respectively.

For quantitative analysis by flow cytometry, the cells were trypsinized by

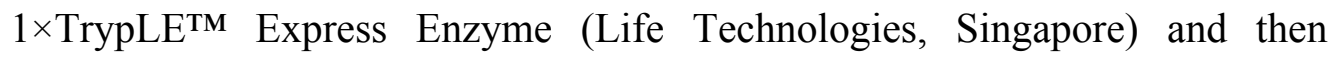
centrifuged. $1 \times$ PBS solution was added into the cell pellet to prepare 
monodispersed cell suspension $(500 \mu 1)$ for flow cytometry (LSR II, BD). The PE channel (excitation: $561 \mathrm{~nm}$; emission: 567-597 nm) was used to detect the fluorescence signals from PCL-DPP-PCL NPs. Regular cells without NP treatment were used as negative control. Approximately $1 \times 10^{4}$ cell events were recorded in each test and the data were analyzed by FlowJo software to obtain the mean fluorescence intensity (MFI) of cell populations. Then the MFI of each labelled group was normalized by the MFI of corresponding control groups, which was able to quantitatively indicate the change of fluorescence intensity before and after cell differentiations.

\subsubsection{Cell proliferation}

The influence of PCL-DPP-PCL NPs on hMSC proliferation was evaluated using PrestoBlue assay. Firstly, both labelled and unlabeled hMSCs were seeded on a 24 -well plate at a density of 2000 cells/well and cultured at $37^{\circ} \mathrm{C}$ with $5 \% \mathrm{CO}_{2}$ for 14 days. The medium was changed every 2-3 days. The absorbance was measured at day $3,7,10$, and 14 . Percentage $(\%)$ reduction that indicate the cell proliferation rate was calculated based on the formula provided by the manufacturer.

\subsubsection{Pre-differentiation processing}

The hMSCs of passage 3 or 4 were cultured in three T75 flasks. When cells became confluent after 3-4 days, PCL-DPP-PCL NPs $(0.2 \mathrm{mg} / \mathrm{mL})$ were added to the culture medium. Regular cells without treatment were regarded as control. After further incubation for 3 and 7 days, the excess NPs were removed away by washing with PBS for three times before the cells were trypsinized by $1 \times$ TrypLE $^{\mathrm{TM}}$ Express Enzyme. The cell suspension in DMEM medium from each group was placed on 4-well plates for osteogenic and adipogenic differentiations respectively, while other cells were seeded in sterile conical tubes for chondrogenic differentiation. The DMEM medium was replaced by differentiation induction medium after one day. 


\subsubsection{MSC differentiations}

$6.0 \times 10^{4}$ and $6.0 \times 10^{3} \mathrm{hMSCs}$ were seeded on $1.9 \mathrm{~cm}^{2}$ Nunc ${ }^{\mathrm{TM}} 4$-well plates (Thermo Fisher Scientific, Singapore) in expansion medium for adipogenic and osteogenic differentiations respectively. Cells were first incubated overnight for effective attachment. Then the expansion medium was replaced by differentiation medium on the following day for induction of adipogenic or osteogenic differentiations. The adipogenic medium comprised high glucose DMEM (without L-glutamine) supplemented with 10\% FBS, $1 \mathrm{mM}$ sodium pyruvate (Life Technologies, Singapore), 100 units/ml penicillin-streptomycin, $0.1 \mu \mathrm{M}$ dexamethasone (Sigma-Aldrich, Singapore), $10 \mu \mathrm{g} / \mathrm{ml}$ insulin (Life Technologies, Singapore), $0.5 \mathrm{mM}$ 3-Isobutyl-1-methylxanthine (IBMX) (Sigma-Aldrich, Singapore) and $200 \mu \mathrm{M}$ indomethacin (Sigma-Aldrich, Singapore). The osteogenic medium included low glucose DMEM, 10\% FBS, $1 \times$ Glutamax $^{\mathrm{TM}}$ (Life Technologies, Singapore), 100 units $/ \mathrm{ml}$ penicillinstreptomycin, $1 \mathrm{mM}$ sodium pyruvate, $50 \mu \mathrm{g} / \mathrm{ml}$ ascorbic acid (Sigma-Aldrich, Singapore), $10 \mathrm{mM} \beta$-glycerophospate (Sigma-Aldrich, Singapore) and $0.1 \mu \mathrm{M}$ dexamethasone. The cells were cultured under $37^{\circ} \mathrm{C}$ under $5 \% \mathrm{CO}_{2}$ humidified conditions with the differentiation medium refreshed every 2-3 days. After adipogenic and osteogenic differentiation, the cells were washed twice by PBS and then trypsinized by $1 \times$ TrypLE $^{\text {TM }}$ Express Enzyme. Then the cells were mixed with DMEM medium and spun down. The cell pellets were resuspended in $500 \mu \mathrm{l}$ PBS for flow cytometry.

Induction of hMSC chondrogenic differentiation was performed in a 3dimensional culture condition. $2.0 \times 10^{5} \mathrm{hMSCs}$ were transferred to a $15 \mathrm{ml}$ conical tube and then centrifuged to form a cell pellet, which was cultured in expansion medium at $37^{\circ} \mathrm{C}$ and $5 \% \mathrm{CO}_{2}$ humidified conditions to allow pellet stabilization. The expansion medium was replaced by chondrogenic medium on the second day. The chondrogenic medium consisted of high glucose DMEM, $1 \times$ Glutamax $^{\mathrm{TM}}, 100$ units/ml penicillin-streptomycin, $1 \mathrm{mM}$ sodium pyruvate, $50 \mu \mathrm{g} / \mathrm{ml}$ ascorbic acid, $0.1 \mu \mathrm{M}$ dexamethasone, $4 \mathrm{mM}$ proline (Life 
Technologies, Singapore) and ITS (BioLab, Singapore) was supplemented with $10 \mathrm{ng} / \mathrm{ml}$ transforming growth factor $\beta 3$ (TGF- $\beta 3$ ) (R\&D Systems, Singapore). The medium was changed every 2-3 days. The chondrogenic pellets after differentiation were firstly homogenized in collagenase type I for $2 \mathrm{~h}$ at at $37^{\circ} \mathrm{C}$ with $5 \% \mathrm{CO}_{2}$ and then spun down. Then $1 \times$ TrypLETM Express Enzyme was added to the pellet for several minutes and cells were centrifuged after adding DMEM medium. Finally, the cells were resuspended in PBS and measured by flow cytometry.

\subsubsection{Histological analysis}

The adipogenic and osteogenic differentiation samples were collected at day 21 , while the chondrogenic pellets were harvested at day 28. The samples were washed with $1 \times$ PBS before being fixed overnight with $10 \%$ formalin (SigmaAldrich, Singapore). The fixed samples were rinsed with $1 \times$ PBS prior to histological staining.

The adipogenic lineage was stained with Oil Red to identify intracellular oil droplet accumulation that can be found in mature adipocytes [137]. The staining solution was prepared by adding $4 \mathrm{ml}$ distilled water to $6 \mathrm{ml}$ of $0.5 \%$ Oil Red O stock solution. After 15 min incubation, the samples were washed with $70 \%$ ethanol and distilled water to remove the excess staining solution before being counterstained with hematoxylin (Sigma-Aldrich, Singapore) for $30 \mathrm{secs}$ and rinsed with distilled water. For osteogenic lineage, Alizarin Red S solution (Sigma-Aldrich, Singapore) was used to stain the cells for $5 \mathrm{~min}$, followed by gentle agitation with distilled water to remove non-specific staining. The cells subjected to osteogenic differentiation presented brick-red color due to calcium deposition. The chondrogenic pellets were embedded in FSC 22 Mounting Media (Leica, Singapore) and frozen at $-20^{\circ} \mathrm{C}$ for about $2 \mathrm{~h}$. The tissue sections of $5 \mu \mathrm{m}$ thick were sliced using a Leica CM1900 cryostat (Leica Microsystems, Singapore) and were collected using Mezel-Glaser Superfrost Plus slides (Thermo Fisher Scientific, Singapore). 
The collagen type II expression was analyzed by immunohistochemistry staining using UltraVision ${ }^{\mathrm{TM}}$ Quanto Detection System HRP DAB kit (Thermo Fisher Scientific, Singapore). The samples on the slides were first incubated with digestive enzyme containing $0.5 \%$ pepsin (Sigma-Aldrich, Singapore) for $20 \mathrm{~min}$ at $37^{\circ} \mathrm{C}$ in humid condition, followed by treatment of hydrogen peroxide for $10 \mathrm{~min}$ and Ultra V Block reagent (Thermo Fisher Scientific, Singapore) for 5 min to reduce non-specific background staining. The samples were then incubated overnight with Mouse monoclonal anti-Col2 antibody (Merck Millipore, Singapore) with a dilution factor of 1:500 at $4^{\circ} \mathrm{C}$. Following the staining protocol of UltraVision ${ }^{\mathrm{TM}}$ Quanto Detection System HRP DAB kit, samples were incubated in Primary Antibody Amplifier Quanto for $10 \mathrm{~min}$, HRP Polymer Quanto for $10 \mathrm{~min}$ with minimum or no light exposure, and finally a mixture of $30 \mu \mathrm{DAB}$ Quanto Chromogen and $1 \mathrm{ml}$ of DAB Quanto Substrate until color development was evident (approximately $5 \mathrm{~min}$ ). The chondrogenic sections were counterstained with hematoxylin (Sigma Aldrich, Singapore). The coverslips were dehydrated after staining.

\subsubsection{Gene expression}

Adipogenic and osteogenic samples were harvested at day 14, while chondrogenic pellets were only collected at day 21. Prior to RNA isolation, chondrogenic pellets were incubated in $0.25 \%$ collagenase type I for $2 \mathrm{~h}$ at $37^{\circ} \mathrm{C}$. The RNA was extracted using RNAeasy mini kit (Qiagen, Singapore) and quantified using NanoDrop 2000 UV-Vis Spectrophotometer (Thermo Fisher Scientific, Singapore). RNA was reverse transcribed into cDNA with $5 \times$ iScript Reverse Trascription Supermix (Biorad Laboratories, Singapore). Real-time Polymerase Chain Reactions (RT-PCRs) were performed with SYBR Green PCR Master Mix kit (Life Technologies, Singapore) using StepOnePlus RealTime PCR System (Life Technologies, Singapore) to quantify the specific gene expression of mRNA in the samples. Samples were first denatured at $95^{\circ} \mathrm{C}$ for $10 \mathrm{~min}$, followed with a 40 -cycle amplification consisting of $95^{\circ} \mathrm{C}$ denaturation step for $15 \mathrm{sec}$ and $60^{\circ} \mathrm{C}$ extension step for $1 \mathrm{~min}$. Primers used to amplify 
cDNA of adipogenic cDNA includes GAPDH, PPAR- $\gamma$ and Leptin. Meanwhile, GAPDH, Col1, OCN and ALP primers were used for osteogenic cDNA, and GAPDH, Col1 and Col2 primers were used for chondrogenic cDNA. The primer sequences are listed in Table 4. The expression levels of the mRNA of target genes were normalized according to the reference gene GAPDH, and then expressed as fold change with reference to their respective levels before induction of differentiation (day 0 ), which is calculated by the widely used comparative $\mathrm{C}_{\mathrm{T}}$ method using the $2^{-\Delta \Delta \mathrm{Ct}}$ formula[138].

Table 4. Primer sequences for specific genes used in RT-PCR. (This table is reprinted with permission from[139]. Copyright (2016) American Chemical Society)

\begin{tabular}{|c|c|c|}
\hline Gene & Forward primer sequence (5'-3') & Reverse primer sequence (5' - 3') \\
\hline $\mathbf{G A P D H}$ & ACAACTTTGGTATCGTGGAAGG & GCCATCACGCCACAGTTTC \\
\hline $\mathbf{P P A R}-\boldsymbol{\gamma}$ & TACTGTCGGTTTCAGAAATGCC & GTCAGCGGACTCTGGATTCAG \\
\hline $\mathbf{L e p t i n}$ & TGCCTTCCAGAAACGTGATCC & CTCTGTGGAGTAGCCTGAAGC \\
\hline $\mathbf{O C N}$ & CGCCTGGGTCTCTTCACTAC & CTCACACTCCTCGCCCTATT \\
\hline $\mathbf{A L P}$ & ACCACCACGAGAGTGAACCA & CGTTGTCTGAGTACCAGTCCC \\
\hline $\mathbf{C o l 1}$ & CAGAACGGCCTCAGGTACCA & CAGATCACGTCATCGCACAAC \\
\hline $\mathbf{C o l} \mathbf{2}$ & CCAGATGACCTTCCTACGCC & TTCAGGGCAGTGTACGTGAAC \\
\hline
\end{tabular}

\subsubsection{Confocal laser scanning microscopy}

The adipogenic and osteogenic lineages (day 21) were washed twice by PBS, fixed by formalin and then permeabilized by $0.1 \%$ Triton-X. Then the samples were blocked by $1 \%$ BSA and stained with Alexa Fluor ${ }^{\circledR} 633$ phalloidin for Factin and DAPI for nucleus. We used three laser channels $(405 \mathrm{~nm}, 561 \mathrm{~nm}$ and $633 \mathrm{~nm}$ ) in a confocal microscope (Carl Zeiss, LSM 710) to excite the fluorophores of DAPI, PCL-DPP-PCL NPs and Alexa Fluor ${ }^{\circledR} 633$ phalloidin. Their fluorescence emission signals were detected in three individual channels (410-549 nm, 566-622 $\mathrm{nm}$ and 638-747 nm). 
For chondrogenic lineage, the cell pellets were washed with PBS twice and fixed with formalin. The fluorescence of the labelled samples were observed by confocal microscope (excitation: $561 \mathrm{~nm}$; emission: 566-622 nm). We applied z-stack function to image different layers of the pellet. Additionally, the cryosection slides of pellets were also imaged with confocal microscopy.

\subsubsection{Cytotoxicity assay}

The cytotoxicity of PCL-DPP-PCL NPs on hMSCs during cell labeling was tested by PrestoBlue assay. Firstly, unlabeled hMSCs were plated on a 96-well plate with density of 6000 cells/well. Then the original medium was replaced with new medium containing $0.2 \mathrm{mg} / \mathrm{mL}$ PCL-DPP-PCL NPs. The cells were incubated with the NPs for 3 and 7 days respectively before the absorbance (measurement wavelength: $570 \mathrm{~nm}$; reference wavelength: $600 \mathrm{~nm}$ ) were measured by a micro-plate reader (Infinite M200 Pro). Cell viability was calculated based on the manufacturer's instructions. The unlabeled hMSCs were regarded as control with relative cell viability as $100 \%$.

\subsubsection{Fabrication of porous scaffold}

Porous scaffold was fabricated using salt leaching and solvent casting techniques. Poly(L-Lactide-co-Epsilon-Caprolactone) (70:30) (Purasorb ${ }^{\circledR}$, Singapore), also known as PLCL, was dissolved in $10 \% \mathrm{w} / \mathrm{v}$ chloroform (Fischer Scientific, analytical grade, Singapore). $\mathrm{NaCl}$ (Sigma-Aldrich) with particle size of 150-200 $\mu \mathrm{m}$ was mixed in the PLCL solvent, which was then casted at a constant rate to form approximately $200 \mu \mathrm{m}$ thick scaffold and dried. The salt component was then leached by washing with water forming a porous scaffold.

The PLCL scaffolds were pretreated with $3 \mathrm{mg} / \mathrm{ml}$ chitosan (Sigma-Aldrich, Singapore) in $4 \mathrm{mM} \mathrm{HCl}$ using aminolysis method [22] before they were soaked in 50\% ethanol and dried. Subsequently, these scaffolds were immersed in $10 \%$ (w/v) 1,6-hexanediamine (Alder BioPharmaceutical, Singapore) in isopropanol (Fischer Scientific, Singapore) and then 1\% glutaraldehyde (Sigma Aldrich, 
Singapore) after washing with $1 \times$ phosphate buffered saline (PBS) (Life Technologies, Singapore). Finally, the scaffolds were incubated in $3 \mathrm{mg} / \mathrm{ml}$ chitosan (Sigma Aldrich, Singapore) in $4 \mathrm{mM} \mathrm{HCl}$ at $4{ }^{\circ} \mathrm{C}$ overnight. Chitosanmodified PLCL scaffolds ( $2 \mathrm{~mm}$ diameter) were sterilized using $70 \%$ ethanol for $2 \mathrm{~h}$ and washed. Approximately $5 \times 104 \mathrm{hMSCs}$ were seeded on each scaffold and cultured.

\subsubsection{Statistical analysis}

All experiments were performed at least thrice. The data were analyzed following Student's t-test to determine the statistical significance between two experimental groups using One-Way ANOVA and Tukey HSD test. The difference between data was considered statistically significant if the p-value was less than 0.05 .

\subsection{Results and Discussion}

For abbreviation, the PCL-DPP-PCL NPs are simply referred to as NPs henceforth. The brief workflow of this study is presented in Figure 18. Firstly, the original hMSCs were incubated with NPs for 3 days (short term, "ST") and 7 days (long term, "LT") with blank cells as control. Then, these labelled cells were induced into adipogenic, chondrogenic and osteogenic differentiation. To study the influence of NPs on hMSC proliferation and multipotency, we divided the MSCs into 3 major experimental groups, i.e., unlabeled control, short-term staining for 3 days (NP-ST) and long-term staining for 7 days (NP-LT).

\subsubsection{Cell labeling with NPs}

We firstly investigated the cellular uptake of NPs. The confocal fluorescence microscopy in both individual channels and ortho-views (Figure 21 A and Figure 19) indicated that NPs were internalized into the cytoplasm of hMSCs, which was consistent to the finding in the previous work [106]. Additionally, prolonged incubation time from 3 days to 7 days for cell staining enhanced the cellular intake of NPs with increased fluorescence intensity (Figure 21A), which 
was further verified by flow cytometry showing the peak intensity shift (Figure 21B). The staining process did not show any considerable effect on the cell viability compared with the unlabeled control (Figure 20), indicating that the NPs had minimum cytotoxicity to the hMSCs. For long-term tracking of cell differentiation, the NP labeling is desired to have minimum effect on cell proliferation to maintain the multipotency of hMSCs. We used PrestoBlue assay to measure the reduction ability of live cells for up to 2 weeks (Figure 21C), which indicated the cell proliferation rate. The results revealed that the shortterm labeled group (NP-ST) and long-term labeled group (NP-LT) did not show any inhibition effect on hMSC proliferation as compared to the unlabeled control. Interestingly, the nanoparticle labelling rather enhanced cell proliferation at day 7 and day 14, while the underlying mechanism is still not clear and needs further investigation.

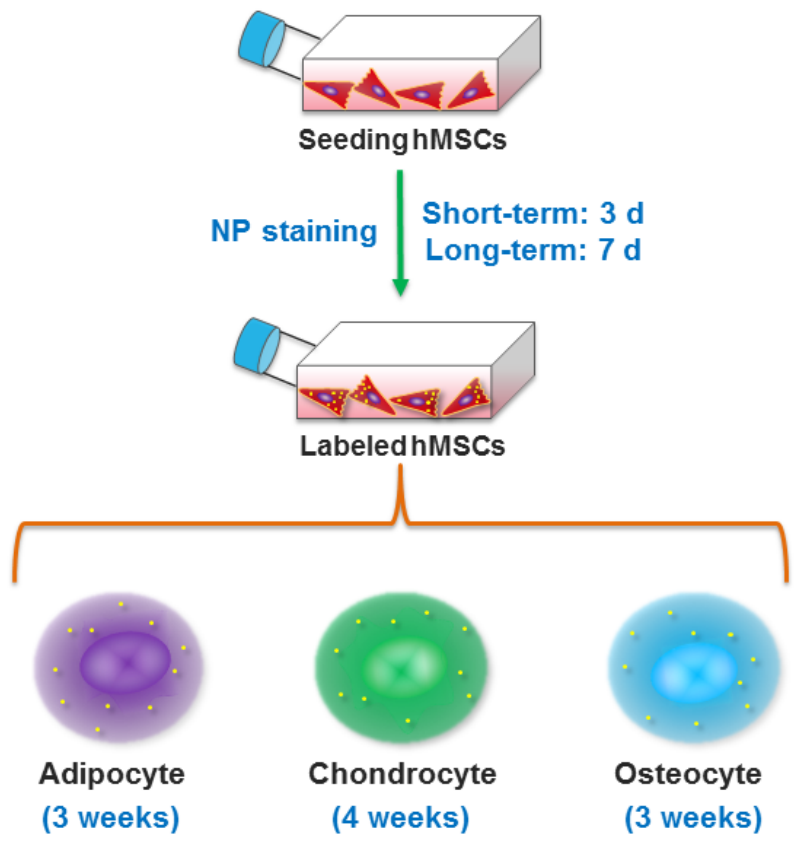

Figure 18. Schematic illustration of long-term tracking human mesenchymal stem cells during adipogenic, chondrogenic and osteogenic differentiations using PCL-DPP-PCL nanoparticles. (This figure is reprinted with permission from[139]. Copyright (2016) American Chemical Society) 

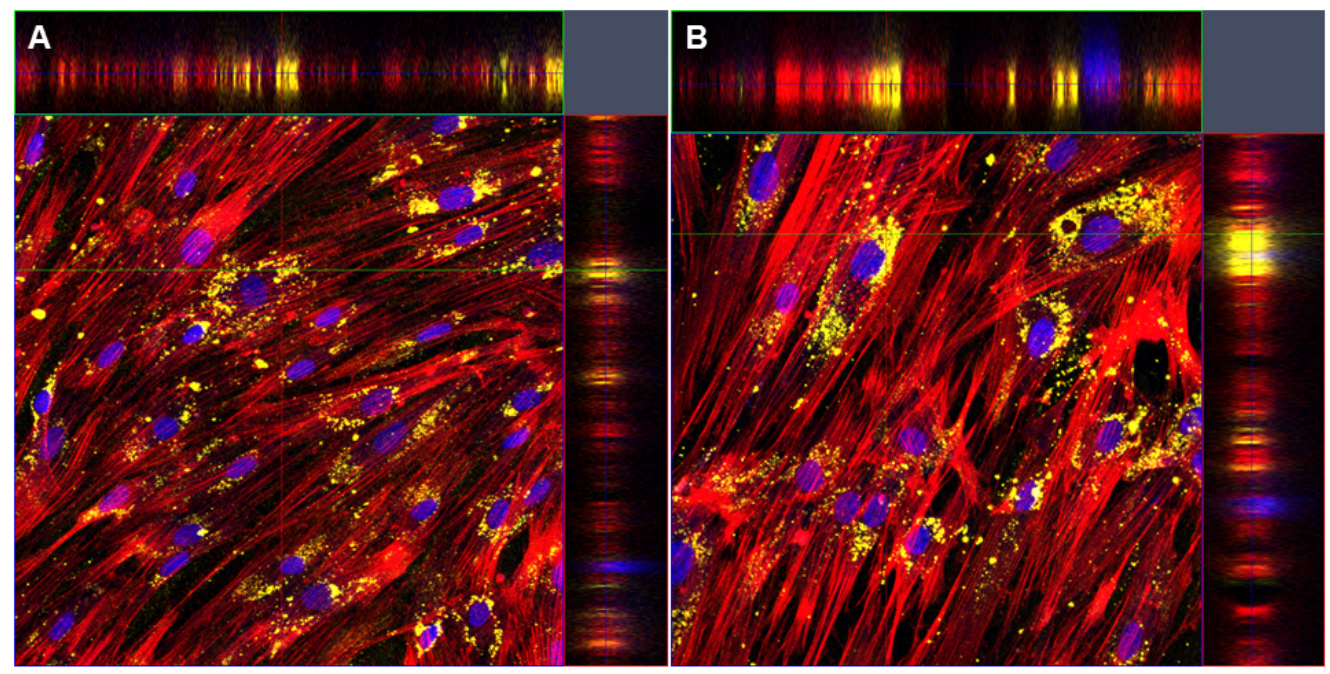

Figure 19. Corresponding ortho-views by taking different $\mathrm{z}$ stacks for the confocal laser scanning microscope images of NP-ST (A) and NP-LT (B) in Figure 21A. Cell nuclei were counterstained with DAPI (blue), F-actin were stained with Alexa Fluor ${ }^{\circledR} 633$ phalloidin (red), and nanoparticles were represented as yellow. (This figure is reprinted with permission from[139]. Copyright (2016) American Chemical Society)

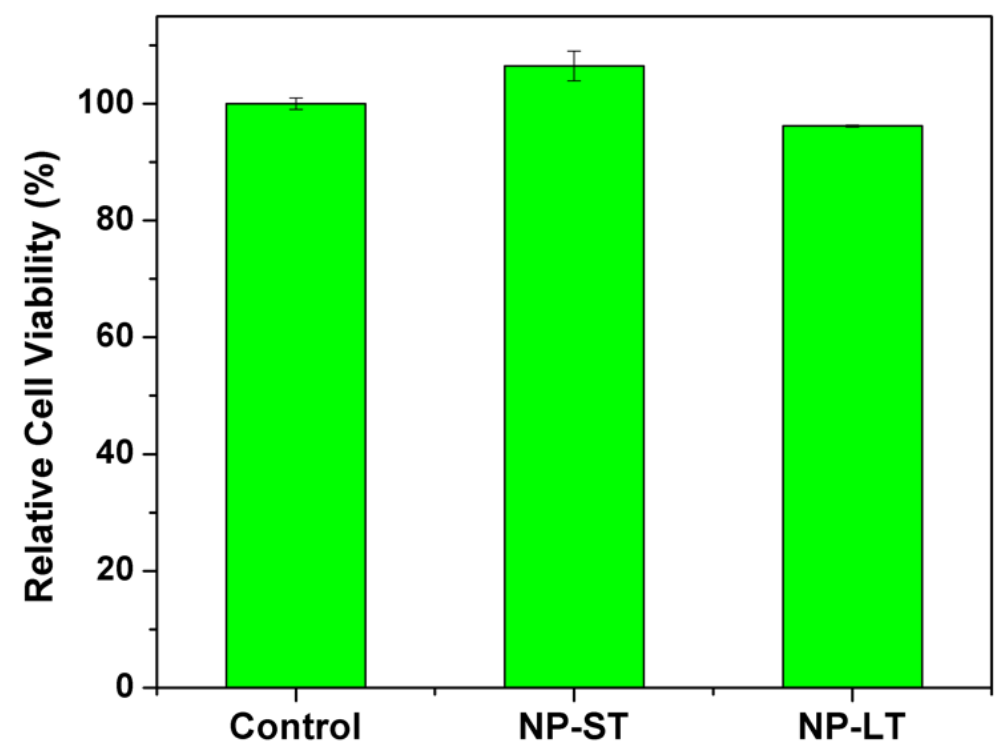

Figure 20. Relative cell viability of hMSCs during NP-labelling (concentration: $0.2 \mathrm{mg} / \mathrm{mL}$ ) detected with PrestoBlue assay. The viability of blank cells without labelling was set as $100 \%$. The data were expressed as means $\pm \mathrm{SD}(\mathrm{n}=3)$. (This figure is reprinted with permission from[139]. Copyright (2016) American Chemical Society) 


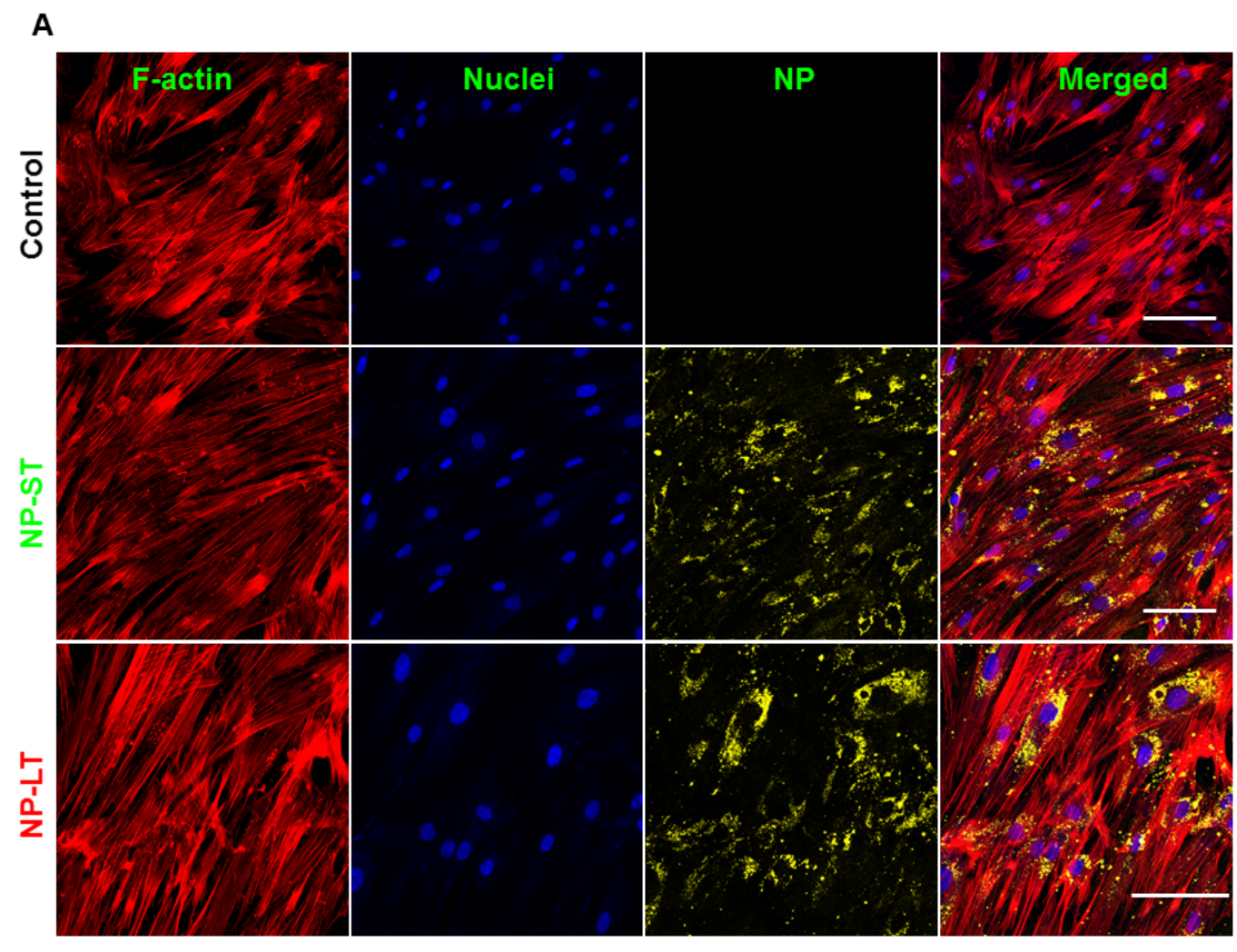

B

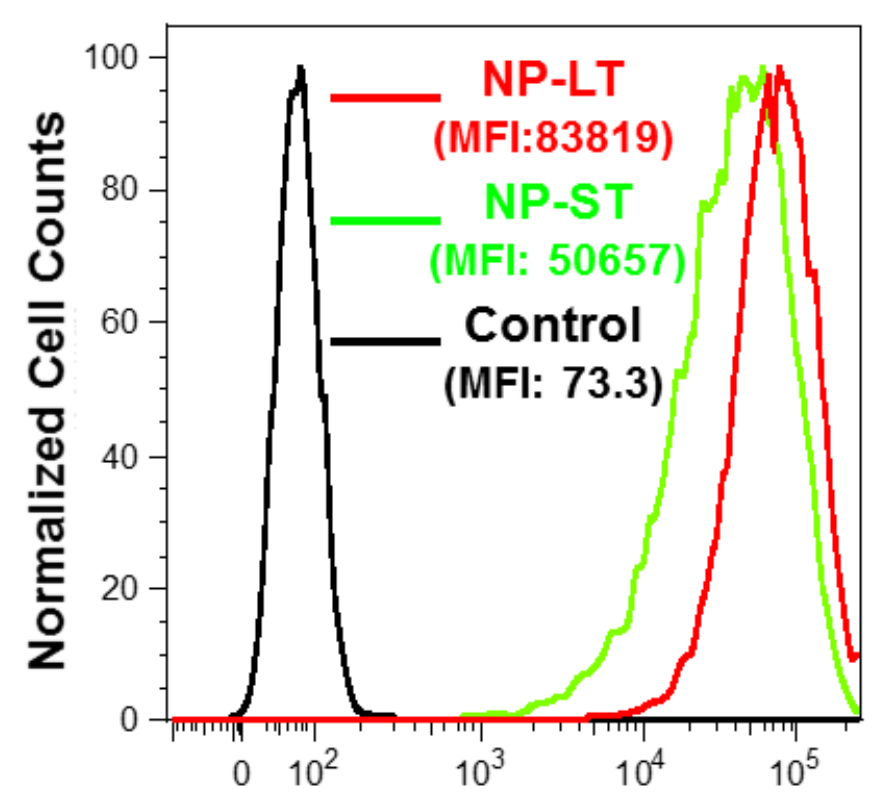

Fluorescence Intensity of NPs 


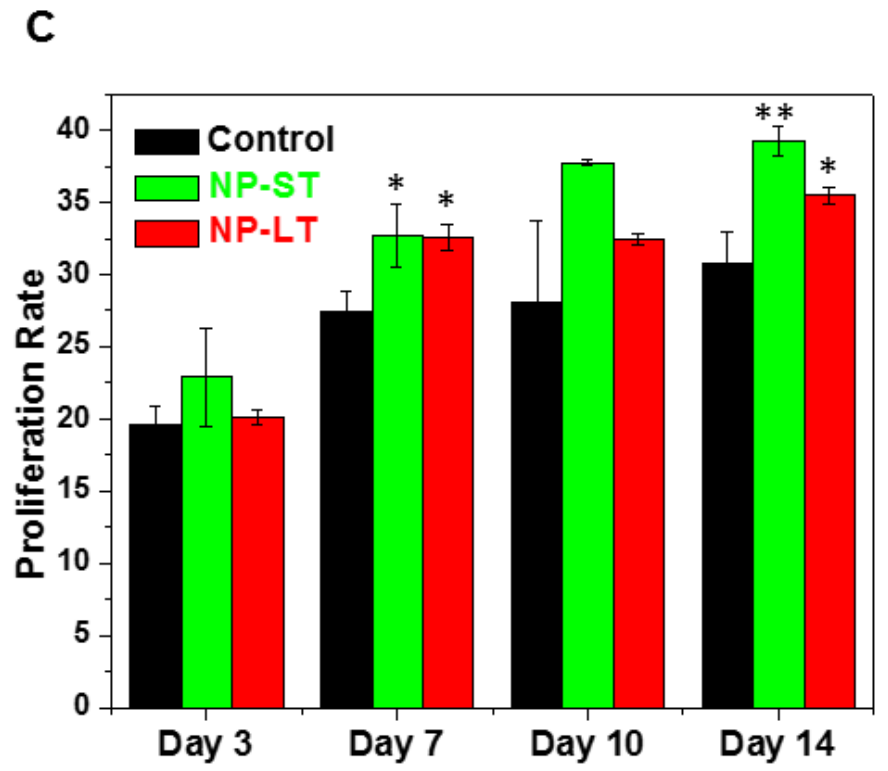

Figure 21. (A) Cellular uptake of NPs in hMSCs imaged by confocal microscopy. Cell nuclei were counterstained with DAPI (blue), F-actin were stained by Alexa Fluor ${ }^{\circledR} 633$ phalloidin (red) and the nanoparticles were represented as yellow. Scale bar: $100 \mu \mathrm{m}$. (B) Quantitative analysis of cellular uptake of NPs by flow cytometry. Normalized MFI: 1143.5 for NP-LT, 691.1 for NP-ST. (C) Cell proliferation measured by PrestoBlue assays. Data presented as mean \pm SD. ${ }^{*}$ p-value $<0.05, * *$ p-value $<0.01 . \mathrm{n}=3$. (This figure is reprinted with permission from[139]. Copyright (2016) American Chemical Society)

\subsubsection{Multilineage differentiations of labeled hMSCs}

Figure 23 showed the long-term effect of NP labeling on hMSC-derived adipogenic differentiation after 3 weeks. The confocal microscopy indicated that differentiated adipocytes retained strong fluorescence due to NP staining (yellow, Figure 23A) for both NP-ST and NP-LT groups.

The observation was further quantified by flow cytometry analysis, which also showed relatively stronger fluorescence of NP-LT than NP-ST (Figure 23B). Although the normalized MFI decreased considerably after 3 weeks of adipogenic differentiation, the NP-labeled adipocytes still exhibited high fluorescence intensity ratio (394.7 for NP-LT, 208.8 for NP-ST) compared to unlabelled controls indicating the strong photostability of the labeling NPs. 
The variation of cell morphology and fluorescence of NP labeling at intermediate stages (day 6,13 and 20) in the adipogenic differentiation were shown in Figure 22. Figure 23D displayed the accumulation of intracellular lipids in the differentiated adipocytes by histological staining, providing the qualitative evidence of successful adipogenesis in the NP-labeled cells. Image analysis of the positive area of Oil Red staining indicated similar levels of lipid formation in the NP-labeled groups as compared with the unlabeled control (Figure 24).

To evaluate the adipogenic differentiation quantitatively, we measured the gene expression of two important adipose biomarkers including leptin and peroxisome proliferator-activated receptor $\gamma(\operatorname{PPAR}-\gamma)$, which regulate fatty acid storage and glucose metabolism. The RT-PCR assay (Figure 23C) revealed that the time of NP staining before induction of adipogenic differentiation influenced the adipose gene expression. Specifically, the NP-ST group exhibited a similar level of leptin and an upregulation of PPAR- $\gamma$ as compared to the unlabeled control. In contrast, the gene expressions of leptin and PPAR$\gamma$ in the NP-LT group were both downregulated significantly. These results implied that prolonged NP staining and higher concentrations of internalized NPs could interfere with the hMSC-derived adipogenic differentiation, for which the underlying mechanism of cellular transduction was still unclear and required further investigation. Nevertheless, we have demonstrated that shortterm NP staining for 3 days (NP-ST) could provide strong fluorescence labeling without compromise of adipogenesis for long-term tracking of hMSC differentiation. Therefore, we applied short-term NP staining in the following chondrogenic and osteogenic differentiations. 


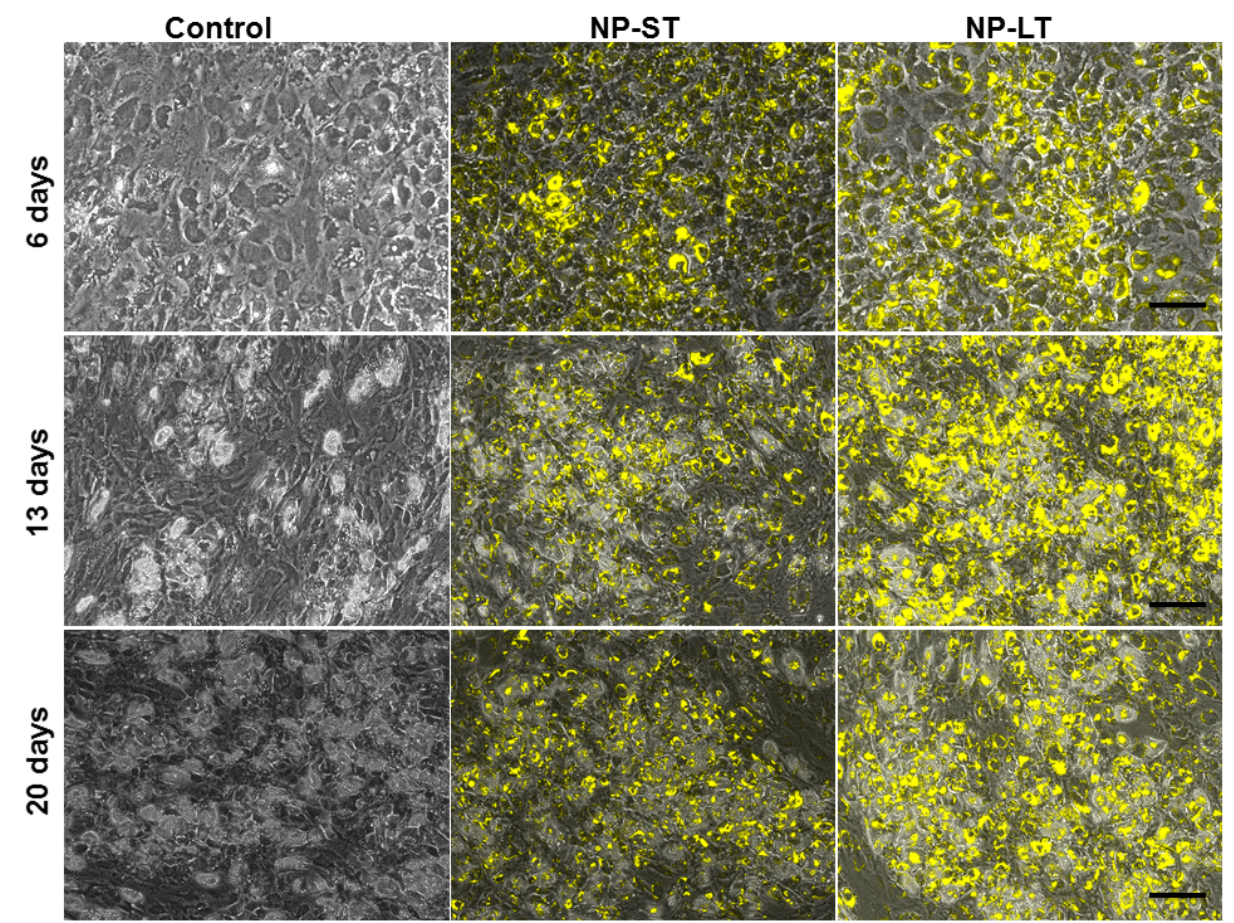

Figure 22. Microscopic images of the NP-labeled hMSCs during adipogenic differentiation. The images of NP-ST and NP-LT groups were obtained by merging the bright field contrast images and the corresponding fluorescent images. Scale bar: $250 \mu \mathrm{m}$. (This figure is reprinted with permission from[139]. Copyright (2016) American Chemical Society)

A

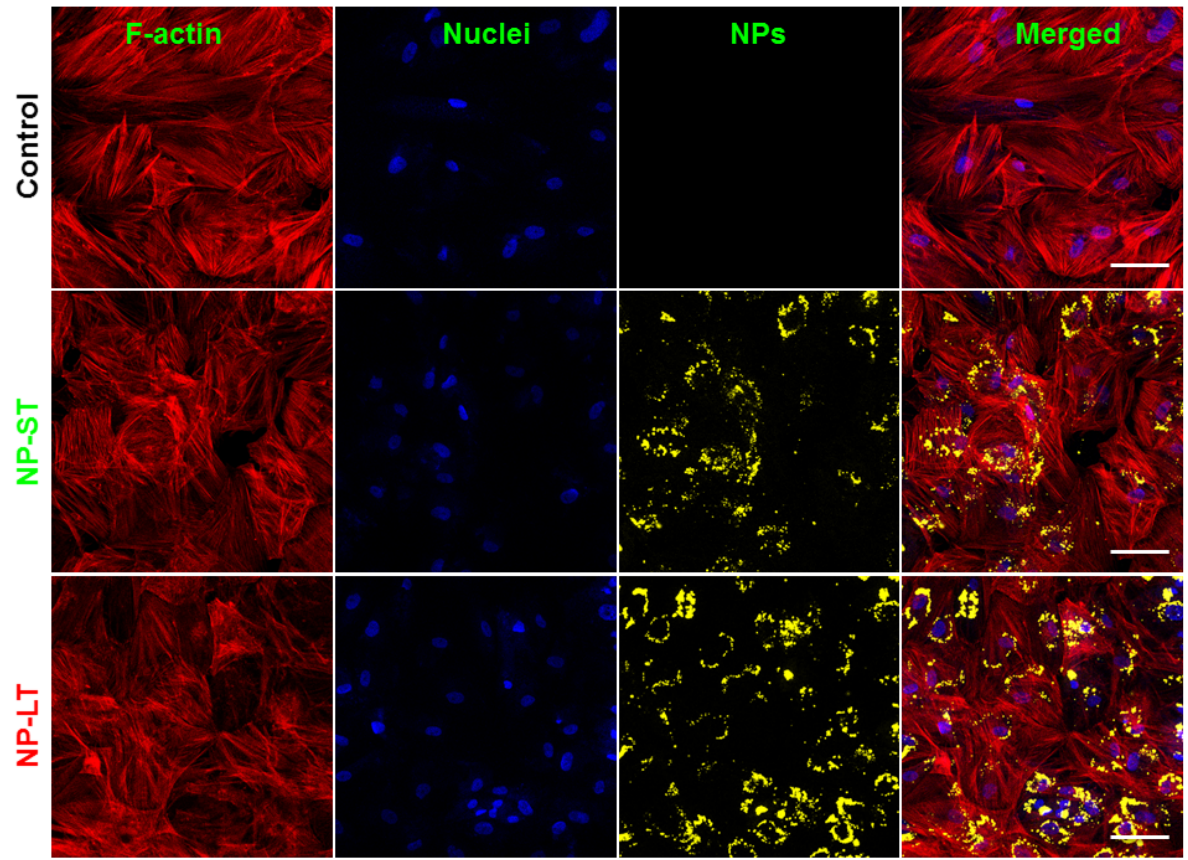




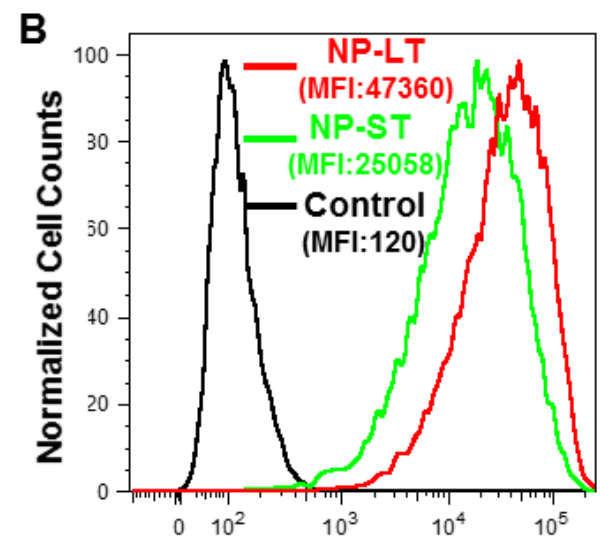

Fluorescence intensity of NPs

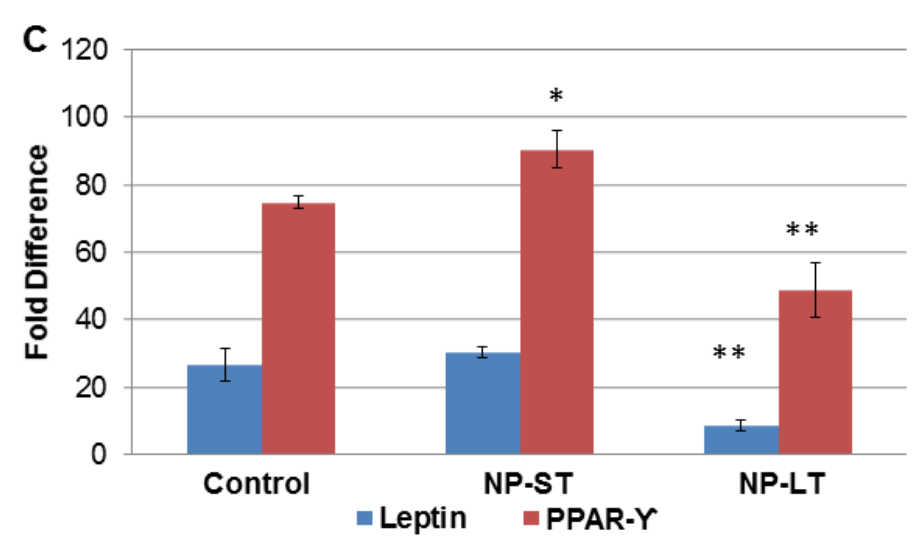

D
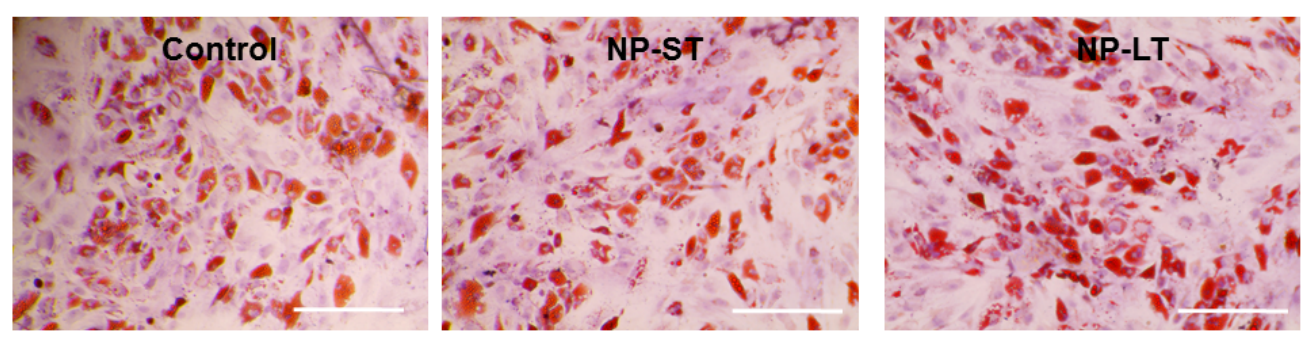

Figure 23. MSC differentiation in adipogenic lineage. (A) Confocal fluorescence images of the differentiated adipocytes (day 21). Scale bar: $100 \mu \mathrm{m}$. (B) The retained fluorescence due to NP staining in the differentiated adipocytes (day 21) analyzed by flow cytometry. Normalized MFI: 394.7 for NP-LT, 208.8 for NP-ST. (C) The mRNA expression of leptin and PPAR- $\gamma$ (day 14) quantified by RT-PCR assay. The results were normalized to the data on day 0 (before differentiation). ${ }^{*}$-value $<0.05,{ }^{* *}$-value $<0.01$. Data presented as mean \pm SD. $\mathrm{n}=4$ for leptin and $\mathrm{n}=3$ for PPAR- $\gamma$. (D) Oil Red staining for oil droplets in the differentiated adipocytes (day 21). Scale bar: $250 \mu \mathrm{m}$. (This figure is reprinted with permission from[139]. Copyright (2016) American Chemical Society) 


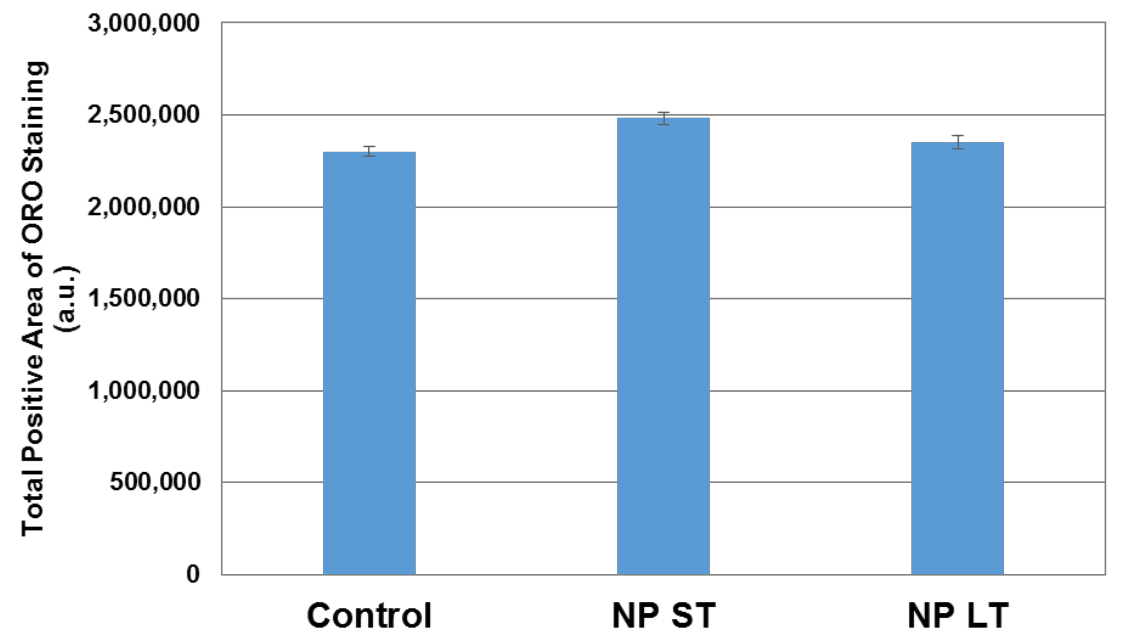

Figure 24. Analysis of the total positive area of Oil Red O (ORO) staining against the reference area of the image frame according to a threshold range set with ImageJ. There is no significant difference among three groups ( $\mathrm{p}$-value $>0.05, \mathrm{n}=7$ ). The area is measured based on an arbitrary unit (a.u.). (This figure is reprinted with permission from[139]. Copyright (2016) American Chemical Society)

The chondrogenic lineage was analyzed after 3-4 weeks of induced differentiation as shown in Figure 25. We observed strong fluorescence within the formed cartilage tissue pellet labeled with NPs (Figure 25B) in addition to its cryosection (Figure 25A). We further homogenized the tissue pellet, digested the matrix proteins and obtained the suspension of chondrocytes for flow cytometry, which showed that the individual hMSC-derived chondrocytes retained strong fluorescence of the NP labels even after 4 weeks of chondrogenesis (Figure 25C).

Three important chondrogenic markers, including aggrecan (AGG), collagen type I (Col1) and collagen type II (Col2), were analyzed using RT-PCR assay for their gene expressions in the chondrogenic pellets at day 21 . The gene expressions of Col1, Col2 and AGG in the labeled chondrogenic pellets were similar to those in the unlabeled control (Figure 25D-E, no significant difference statistically, $\mathrm{p}$-value $>0.05)$. As the most abundant collagen in native cartilage tissues [140], Col2 exhibited very high level of expression (Figure 25E) that led 
to Col2/Col1 ratio above 1,500 (Figure 25F) in both labeled and unlabeled groups, which was a feature of hyaline-like cartilage tissue formation[140, 141].

Immunohistochemistry staining further demonstrated the similar density of Col2 in labelled and control tissue pellets after 4 weeks of chondrogenesis (Figure 25G). Therefore, the NP-labelling was also proved to be amicable for long-term tracking of chondrogenic differentiation of hMSCs.
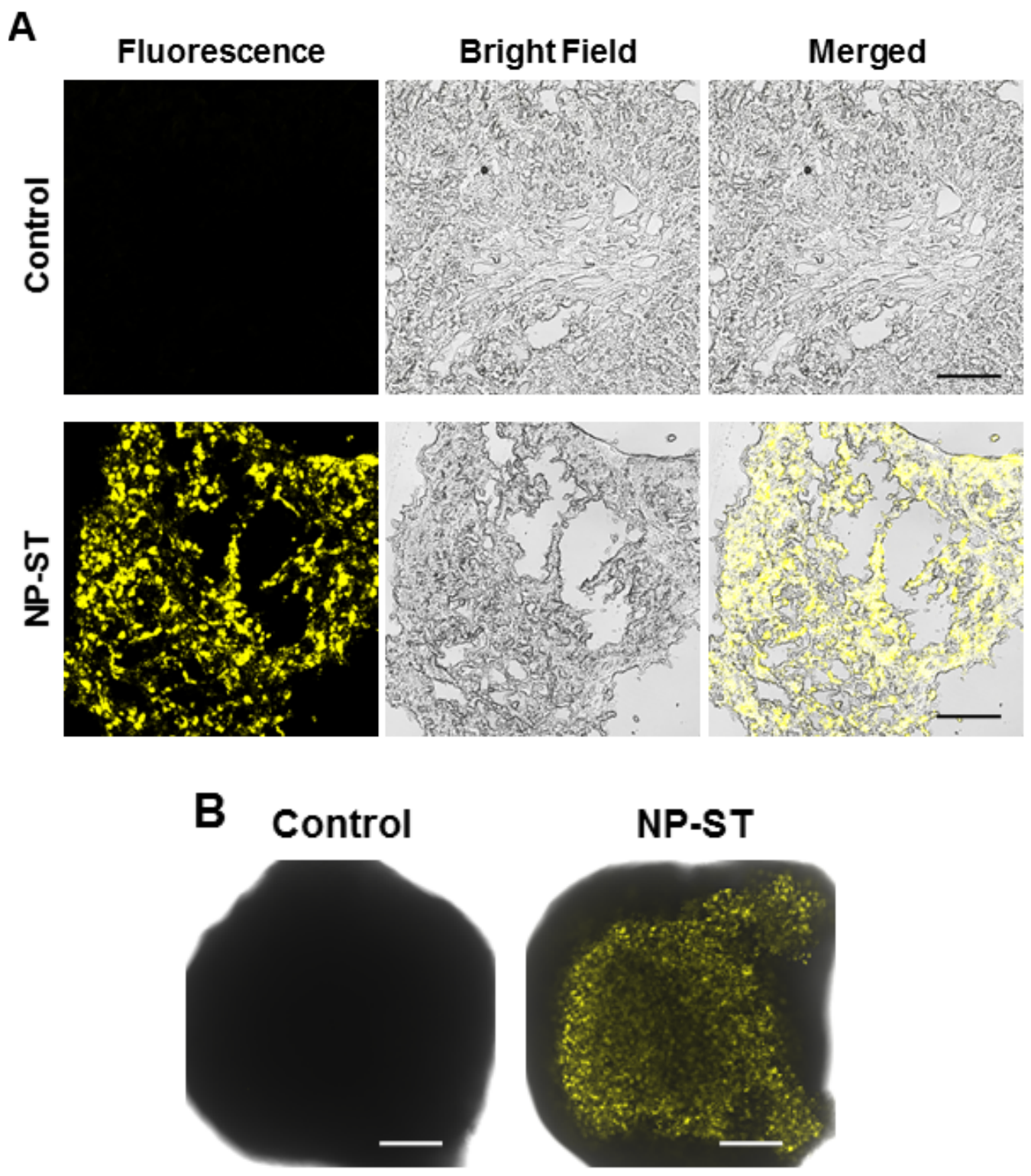


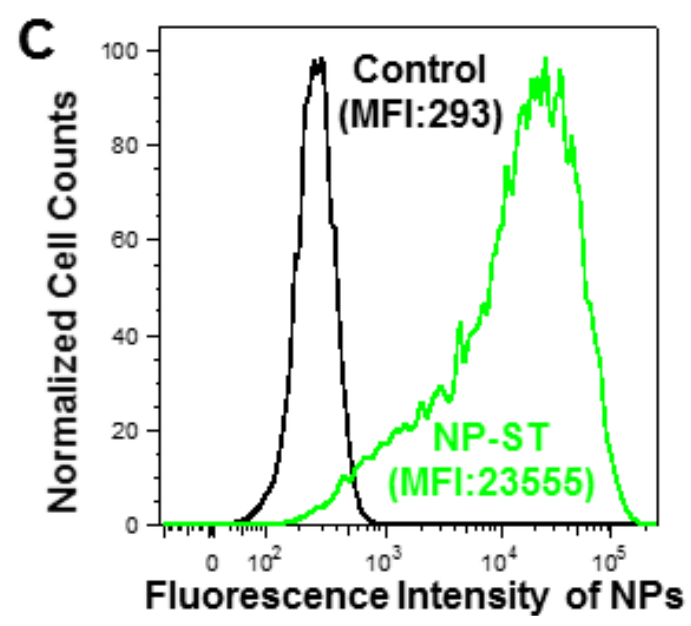

D

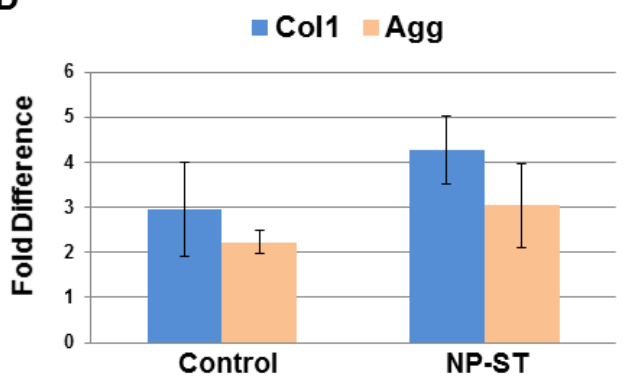

E

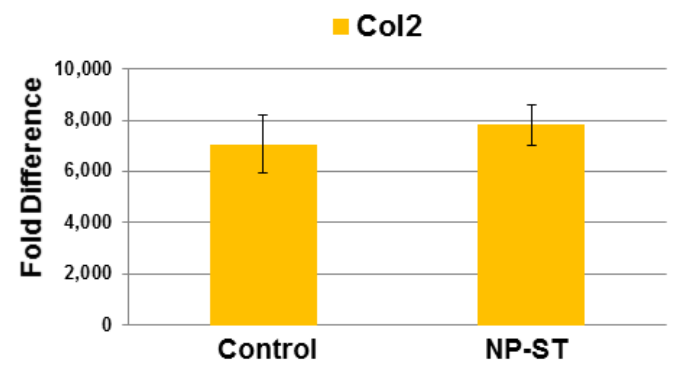

$\mathbf{F}$

$\triangle \mathrm{Col} 2 / \mathrm{Col} 1$

G
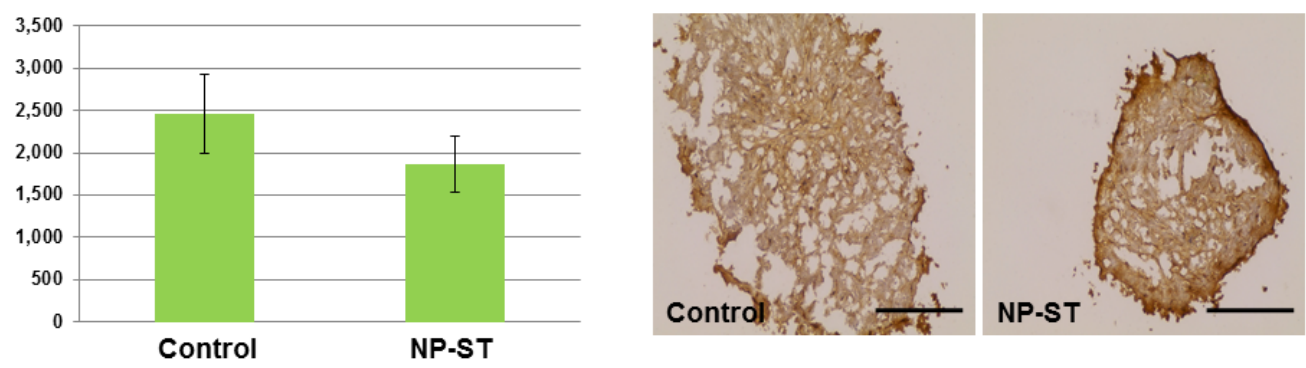

Figure 25. MSC differentiation in chondrogenic lineage. (A) The cryosections of the hMSCderived tissue pellets (day 28). Scale bar: $100 \mu \mathrm{m}$. (B) Confocal fluorescence images of the original pellets (day 28). Scale bar: $200 \mu \mathrm{m}$. (C) Flow cytometry analysis of the trypsinized chondrocytes (day 28). Normalized MFI: 80.4 for NP-ST. (D-F) The mRNA expression of Col1, Col2 and aggrecan (day 21) quantified by RT-PCR assay. Data were normalized according to the results at day 0 (before differentiation). Data presented as mean \pm SD. $n=5$ for Col1, Col2 and Col2/Col1, $n=3$ for AGG. (G) Immunohistochemistry staining of Col2 in the chondrogenic pellets (day 28). Scale bar: $250 \mu \mathrm{m}$. (This figure is reprinted with permission from[139]. Copyright (2016) American Chemical Society) 
The last differentiated group was the osteogenic lineage. Confocal fluorescence microscopy after 3 weeks of osteogenic differentiation (day 21) indicated strong fluorescence in the hMSC-derived osteocytes (Figure 26A,D). However, the NP-labelled osteocytes exhibited much lower intensity of Alizarin Red staining as compared to the unlabelled control (Figure 26B), suggesting that the presence of NPs suppressed the calcium deposition considerably during osteogenesis.

We also quantified the gene expressions of three important osteogenic markers, including Col1, osteocalcin (OCN) and alkaline phosphatase (ALP), using RTPCR assay at day 14 after induction of differentiation. The RT-PCR data revealed significant downregulation of all three osteogenic gene markers in the NP-labelled group (Figure 26C). These results implied that the NP labelling, even by short-term staining of 3 days, had inhibited the osteogenic differentiation of hMSCs. It was previously reported that $\mathrm{TiO}_{2}$ nanoparticles exhibited adverse effects on the viability, proliferation and osteogenic differentiation of MSCs by influencing the gene expressions of ALP, OCN and osteopontin (OPN) in a size-dependent and dose-dependent manner [142]. In the present study, on the other hand, we showed that the synthetic polymerbased NPs with staining concentration of $0.2 \mathrm{mg} / \mu \mathrm{L}$ did not affect the cell viability and proliferation, while they compromised the osteogenic differentiation of MSCs.
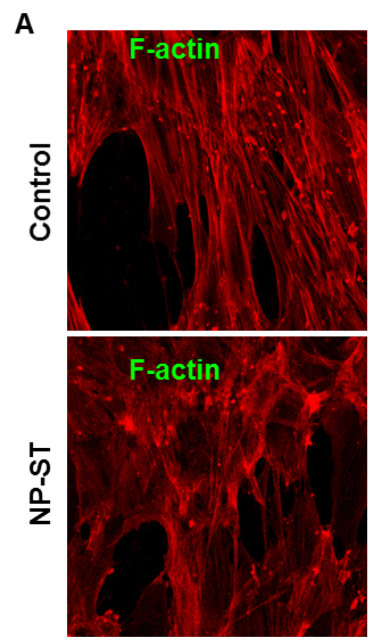
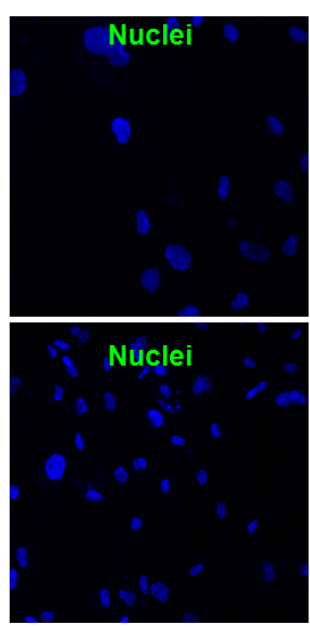
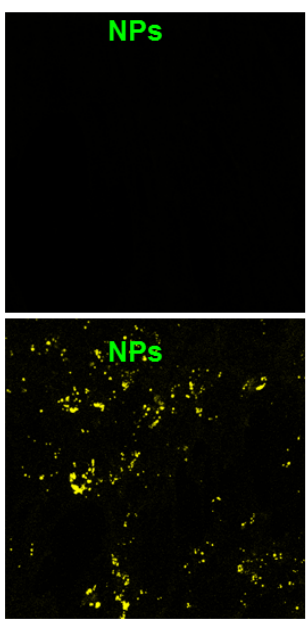

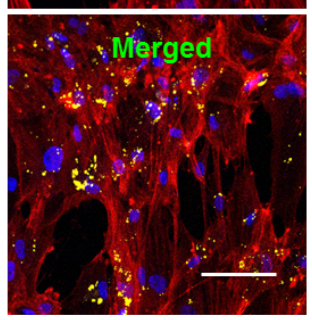



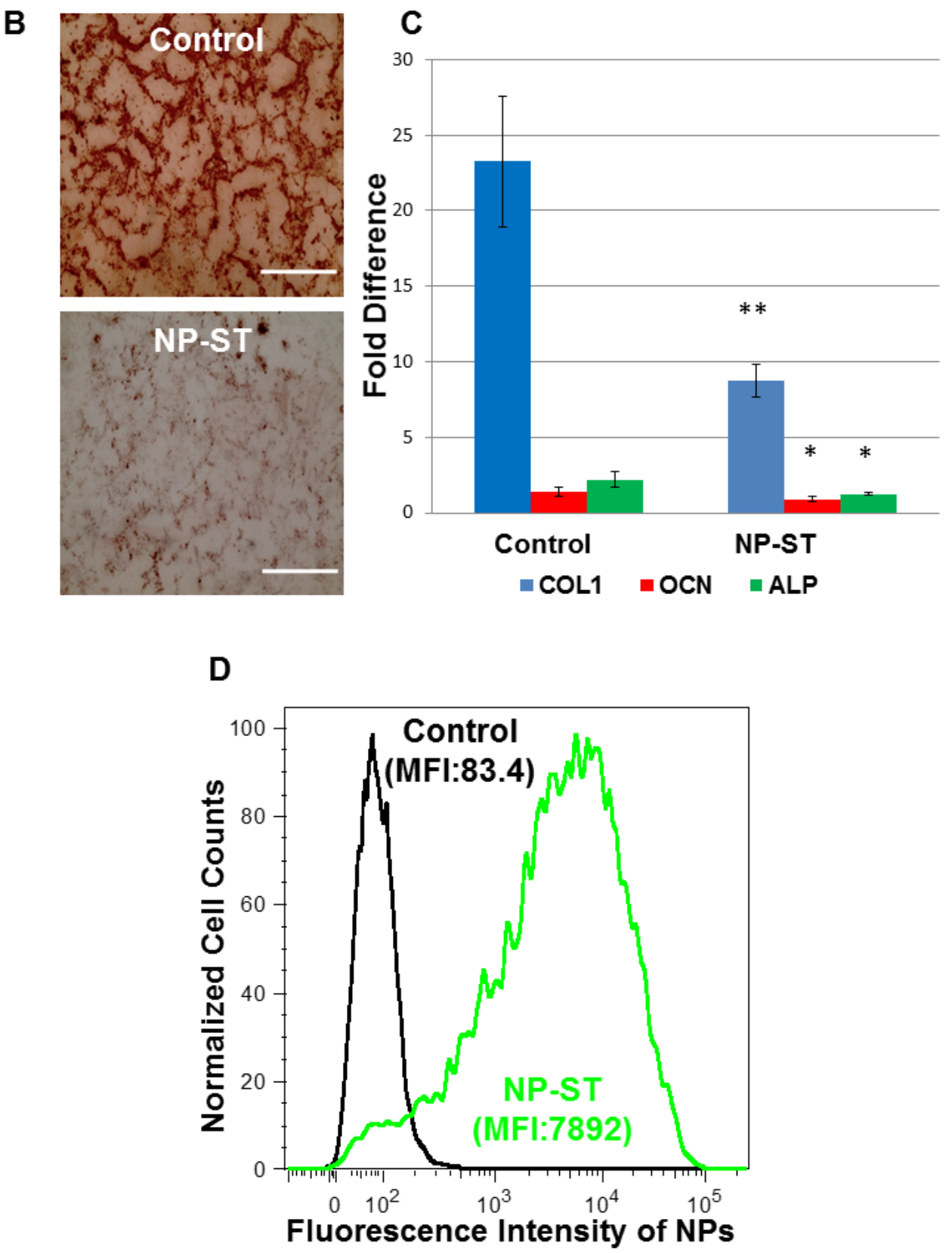

Figure 26. MSC differentiation in osteogenic lineage. (A) Confocal fluorescence images of the differentiated osteocytes (day 21). Scale bar: $100 \mu \mathrm{m}$. (B) Alizarin Red histological staining for calcium deposition after osteogenic differentiation (day 21). Scale bar: $250 \mu \mathrm{m}$. (C) The mRNA expression of Col1, OCN and ALP (day 14) quantified by RT-PCR assay. Data were normalized according to the results at day 0 (before differentiation). ${ }^{*} \mathrm{p}$-value $<0.05,{ }^{*}$ p-value $<0.01$. Data presented as mean \pm SD. $n=4$. (D) Flow cytometry analysis of the hMSC-derived osteocytes (day 21). Normalized MFI: 94.6 for NP-ST. (This figure is reprinted with permission from[139]. Copyright (2016) American Chemical Society) 


\subsubsection{MSC labeling in 3D scaffold}

All above results were based on MSC labelling for conventional 2-dimensional (2D) cell cultures or pellet culture. In the recent advances of tissue engineering, 3-dimensional (3D) scaffolds have become more popular due to many advantages in better mimicking the native cellular environment, which consists of a 3D network with a complex extracellular environment and a highly porous nanotopography [143].

Cells cultured in 3D scaffolds tend to behave more like their in-vivo counterparts, which may work as a better model for tissue engineering and other applications such as drug discovery and cellular signalling [144]. The special mechanical properties of these 3D scaffolds also provide supports to the cells and newly formed tissues, which is important for weight-bearing tissues such as cartilage and bone $[136,145]$.

As a simple proof-of-concept study, we seeded the hMSCs on a Poly(L-Lactideco-Epsilon-Caprolactone) (PLCL) porous scaffold (Figure 27) and cultured the cells for 14 days. The 2D and 3D images in Figure 28 obtained by confocal fluorescence microscopy demonstrated the distribution of NP-labelled MSCs scattered in the scaffold.

The cell nuclei stained with DAPI was indicated as blue dots in the magnified inset of Figure 28. It was however difficult to distinguish the nuclei from the strong background fluorescence under excitation of $405 \mathrm{~nm}$ due to the autofluorescence of PLCL scaffold [146]. Meanwhile, the bright fluorescent NPs clearly indicated the distribution of labelled hMSCs in the scaffold under excitation of $561 \mathrm{~nm}$. This simple test showed that these NPs could also be used as an excellent contrast agent for cell tracking in the 3D scaffold environment, providing important information on the location, distribution and migration of the labeled cells. 

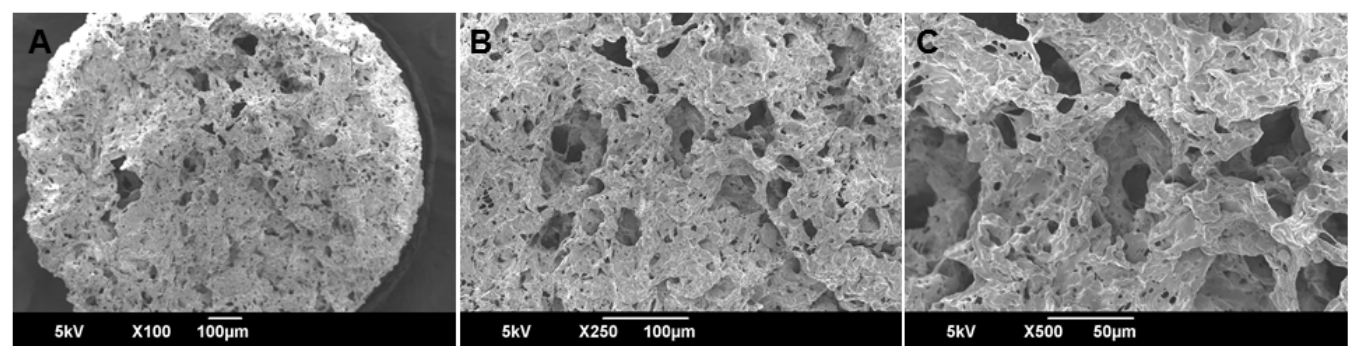

Figure 27. Scanning electron microscope images for PLCL porous scaffold under different magnifications: (A) $100 \times$, (B) $250 \times$ and (C) $500 \times$.(This figure is reprinted with permission from[139]. Copyright (2016) American Chemical Society)

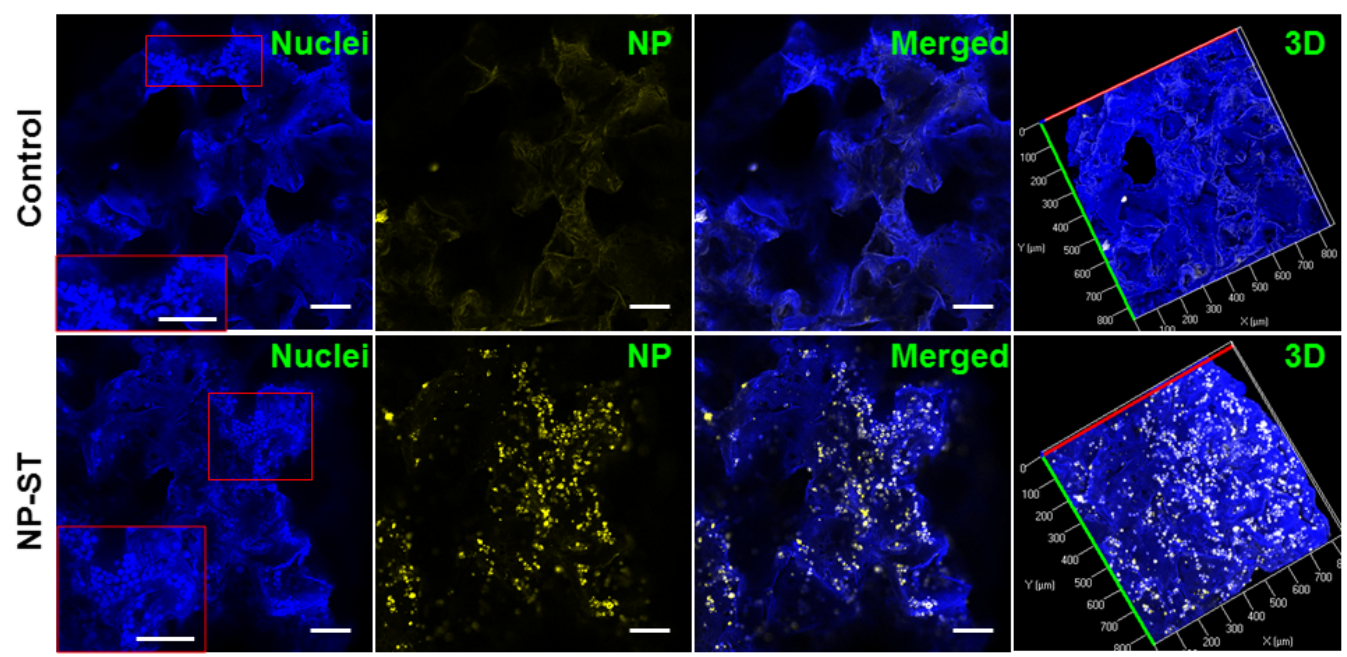

Figure 28. Confocal fluorescence images of hMSCs seeded in a 3D scaffold (day 14). 3D images were reconstructed using ZEN 2012 software by taking different z-stack images. Scale bar: 100 $\mu \mathrm{m}$. (This figure is reprinted with permission from[139]. Copyright (2016) American Chemical Society)

\subsection{Conclusions}

In summary, PCL-DPP-PCL nanoparticles are able to label hMSCs by internalization without affecting the cell viability and proliferation behaviors. It was found that PCL-DPP-PCL NPs labeling for 3 days at concentration of 0.2 $\mathrm{mg} / \mu \mathrm{L}$ did not show any negative effect on MSC adipogenic and chondrogenic differentiations, while the osteogenic differentiation was compromised compared to the unlabeled control. Furthermore, the labeling NPs can maintain 
strong fluorescence even after 4 weeks, indicating these NPs can work as efficient fluorescent probes for long-term tracing during adipogenic and chondrogenic differentiations. The PCL-DPP-PCL nanoparticles do not require any additional bioconjugation or surface modification, while showed minimum cytotoxicity and negative effects on adipogenic and chondrogenic differentiations under appropriate concentrations. Moreover, the PCL polymer skeleton of this probe has proved to be biocompatible and biodegradable, and has been approved by US Food and Drug Administration (FDA) for specific use in human body. Whereas the potential toxic fluorophore DPP composes only about $1.3 \%$ in weight fraction of this probe, thereby further reducing its potential cytotoxicity. By replacing DPP with other near infrared dye in this system, this strategy could be extended for long-term stem cell tracking applications in vivo.

(This chapter is reprinted with permission from[139]. Copyright (2016) American Chemical Society) 


\section{Chapter 4: Peptide Delivery with Poly (ethylene glycol) Diacrylate Microneedles for Keloid Scar Treatment}

\subsection{Introduction}

In addition to long-term stem cell tracking problem, there is another tough issue in the regenerative medicine field, which is keloid scar treatment for skin regeneration. Keloid scars, as a complex abnormal scar, cause not only aesthetic disfigure to the patients, but also have leaded to pruritus, infection, and much distress. Traditional measures to help management of keloids, such as surgery removal, intralesional injection of drugs, or silicon sheet dressing, may cause pain, infection, and less compliance to patients. High rate of recurrence and frequent follow-up result in many inconveniences. Therefore, it's very meaningful to develop a non-invasive method to deliver the drugs which patients can apply by themselves.

Microneedles are emerging as a proficient transdermal delivery system. It allows a variety of molecules to be transported into skin in a non-invasive way, which overcomes the limitations of conventional needle injection[147-149]. Polymer-based microneedles are specifically attractive due to their excellent biocompatibility, biodegradability and nontoxicity[150-152]. Drugs can be loaded into the microneedle tips and/or base, or coated on the microneedle tips[147, 150, 151]. One example is poly(ethylene glycol) diacrylate (PEGDA) based microneedles[153-155]. PEGDA is biocompatible and can be crosslinked in a short time under UV exposure (a few seconds), which facilitates the control of morphology and dimension of microneedles[153, 156]. Small molecule drugs like 5-fluororacil (5-FU) and curcumin have been integrated within microneedles during the fabrication process[157], in which drugs are mixed with the monomer before the mixture is placed in a mold and exposed to UV light. For example, our group has utilized PEGDA-made microneedles to deliver the 5-FU for treating keloid scar cells[158]. PEGDA microneedles have 
sufficient mechanical strength to penetrate skin and release drugs to inhibit keloid fibroblast growth. In addition, other small molecule drugs, such as hydrophobic campothecin (CPT) - that targets DNA topoisomerase I (Topo I) and inhibit collagen synthesis[43] can also be encapsulated and delivered in a similar manner.

In recent years, peptide-based therapeutics have played a major role in new drug development[159-162]. However, this process has not been without difficulties. Peptides face hurdles including instability under high temperature, light, and high/low pHs, and digestion by gastrointestinal enzymes during oral delivery. Microneedles have been suggested as a means to overcome this delivery hurdle since it can bypass oral uptake routes. The easiest way to load the peptide is to coat them on the surface of needles, however, it often requires optimization and adjusting the coating formulations by adding excipients to increase viscosity and decrease surface tension in order to achieve uniform and sufficient coating[93]. Preloading drugs into the needles during the fabrication is an alternative way. However, preloading processes should not disrupt or significantly compromise peptide activity. PEGDA microneedles have been used to deliver several small molecular drugs that are pre-loaded into the polymer matrix during the fabrication. However, this procedure is unsuitable for peptides since high energy UV rays can denature peptides thus compromise the biological activity of peptide-based therapeutics[163, 164].

This chapter introduces a gentle strategy to load peptides into the PEGDA microneedles without the above complications based on the swelling ability of PEGDA in aqueous solution. We discovered that substances with molecular mass less than $4 \mathrm{kDa}$ readily enter the PEGDA matrix during swelling in aqueous solutions. Furthermore, this process is dependent on the period of UV exposure to crosslink PEGDA monomers (part of the microneedle fabrication process). As a proof of concept, the model peptide (Gap 26, a connexin mimetic peptide that inhibits cellular gap-junction)[80, 81, 84, 86, 87, 165-167], is 
loaded into the PEGDA microneedles using this PEGDA swelling strategy (Figure 29). After examining the loading capacity and release profile of the peptide-loaded microneedles, the potential therapeutic effect is explored on a keloid scar model comprising of cells and ex vivo skin.

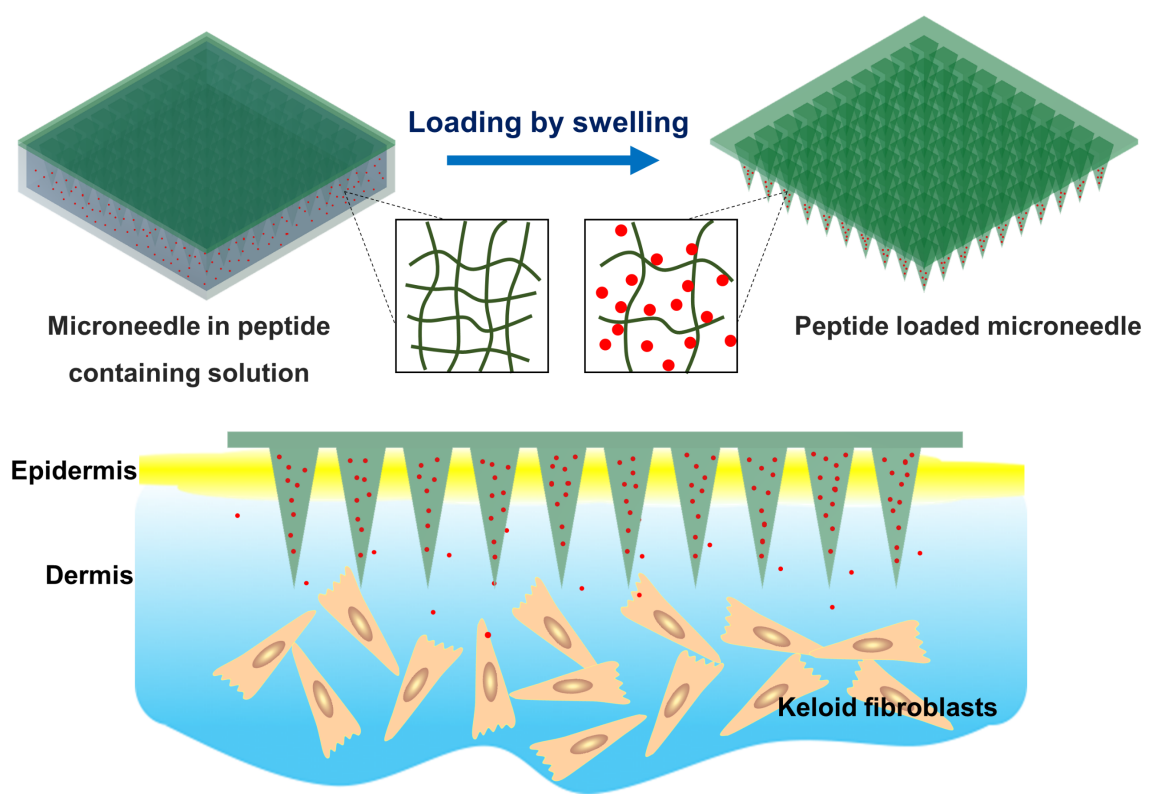

Figure 29. Schematic showing the loading of hydrophilic peptides into poly(ethylene glycol) diacrylate microneedles by the swelling effect. (This figure is reprinted with copyright from[168])

\subsection{Materials and Methods}

\subsubsection{Materials}

Fluorescein Diacetate (FDA), Calcein-AM, Vybrant ${ }^{\circledR}$ DiI Cell-Labeling Solution, and Geltrex ${ }^{\mathrm{TM}}$ LDEV-Free Reduced Growth Factor Basement Membrane Matrix were purchased from Thermo Fisher Scientific Inc. Goat Anti-Mouse $\operatorname{IgG}(\mathrm{H}+\mathrm{L})$ Antibody and Rhodamine conjugate were from Sigma Aldrich. Keloid fibroblasts (KF110) were ordered from Cell Research Corporation (Singapore). Dulbecco's modified eagle medium (DMEM) (w/4.5 $\mathrm{g} / \mathrm{L}$ D-glucose, w/phenol red), fetal bovine serum (FBS), penicillin- 
streptomycin (10,000 U/ml), Phosphate buffered saline (1x, PBS), and trypsinEDTA $(0.25 \%, 10 x)$ were obtained from Gibco Life Technologies (USA). All reagents were of analytical reagent grade and used without further purification.

\subsubsection{Fabrication of blank and CPT loaded PEGDA microneedles}

Firstly, a negative polydimethylsiloxane (PDMS) mold of microneedles was prepared by curing PDMS (base: curing agent $=10: 1$ ) on the stainless microneedle template for $3 \mathrm{~h}$ at $70{ }^{\circ} \mathrm{C}$. In order to make blank PEGDA microneedles, the mixed solution of PEGDA (MW=250) and photoinitiator 2hydroxy-2-methylpropiophenone (HMP) $(0.5 \% \mathrm{v} / \mathrm{v})$ was added to the negative PDMS mold pre-treated with plasma. The gas bubbles in the solution were removed by vacuum before the whole sample was exposed to UV (Intensity: 10 $\mathrm{mW} / \mathrm{cm}^{2}$ ) for crosslinking. Finally, PEGDA microneedles patch was peeled off from the PDMS mold.

The CPT loaded PEGDA microneedles were fabricated in a similar way as the blank microneedles except that the CPT was present in PEGDA solution. CPT loaded microneedles were imaged by confocal laser scanning microscope (Carl Zeiss, LSM 710) with excitation at $405 \mathrm{~nm}$ laser and emission at $410 \mathrm{~nm} \sim 585$ nm. Mechanical properties of microneedles were measured by Instron 5543 Tensile Meter with a speed of $0.5 \mathrm{~mm} / \mathrm{min}$.

\subsubsection{Swelling ratio and mesh size determination}

The microneedle patches were placed in distilled water for $22 \mathrm{~h}$. Then the samples were taken out and gently dried on the paper, and the weight in the swollen state $\left(\mathrm{W}_{\text {swell }}\right)$ was obtained. Later, the patches were transferred into an oven preheated to $50{ }^{\circ} \mathrm{C}$ and dehydrated until a stable dry weight $\left(\mathrm{W}_{\mathrm{dry}}\right)$. The swelling ratio can be calculated by equation (1).

$$
Q=\frac{W_{\text {swell }}-W_{\text {dry }}}{W_{d r y}} \times 100 \%
$$


To calculate the mesh size [169], the polymer volume fraction of the swollen state $v_{2 s}$ was firstly obtained by equation (2).

$$
v_{2 s}=\frac{1}{Q \frac{\rho}{\rho_{H_{2 O}}}+1}
$$

where $Q$ is the swelling ratio, $\rho$ is polymer density, and $\rho_{\mathrm{H}_{20}}$ is water density.

The average molecular weight between two consecutive crosslinks $\left(\mathrm{M}_{c}\right)$ can be calculated by equation (3), where $M_{n}$ is the average molecular weight of the starting polymer $(250 \mathrm{~g} / \mathrm{mol})$ here, $V_{1}$ is the molar volume of water (18 $\left.\mathrm{cm}^{3} / \mathrm{mol}\right), \chi$ is the Flory-Huggins polymer solvent interaction parameter and assumed equal to $0.426, v_{2 r}$ is the polymer volume fraction in the relaxed state (i.e. right after crosslinking and before full swelling), and the polymer volume fraction in the precursor solution was assumed as indicative of $v_{2 r}$.

$$
\frac{1}{M_{c}}=\frac{2}{M_{n}}-\frac{\frac{1}{V_{1}}\left[\ln \left(1-v_{2 s}\right)+v_{2 s}+\chi v_{2 s}^{2}\right]}{\rho v_{2 r}\left[\left(\frac{v_{2 s}}{v_{2 r}}\right)^{\frac{1}{3}}-\frac{1}{2}\left(\frac{v_{2 s}}{v_{2 r}}\right)\right]}
$$

Finally, mesh size can be calculated by the equation (4).

$$
\xi=l\left(2 \frac{M_{c}}{M_{r}}\right)^{\frac{1}{2}} C_{n}^{\frac{1}{2}} v_{2 s}{ }^{-\frac{1}{3}}
$$

where $l$ is the bond length $\left(1.5 \mathrm{~A}^{\mathrm{o}}\right), \mathrm{M}_{\mathrm{r}}$ is the molecular weight of the PEG repeating unit ( $44 \mathrm{~g} / \mathrm{mol}$ ), and $C_{n}$ is the characteristic ratio for PEG, equal to 4 .

\subsubsection{Fabrication of hydrophilic molecules (FITC, FITC-Dextran, Gap 26) loaded PEGDA microneedles}

Aqueous solutions containing FITC and FITC-Dextran with different molecular weight (i.e. $4 \mathrm{kDa}, 10 \mathrm{kDa}, 20 \mathrm{kDa}$, and $70 \mathrm{kDa}$ ) were prepared with DI water and diluted to have the same FITC fluorescence intensity. A specific reservoir with flat bottom was made similarly by PDMS using the stainless microneedles to 
allow one patch of microneedles to be immersed in the hydrophilic solution (100 $\mu \mathrm{L}$ ) for loading. After $22 \mathrm{~h}$ incubation, the microneedle patch was washed with water twice to remove the molecules on the surface before being dried naturally. The FITC and FITC-Dextran loaded microneedle patches were imaged by confocal laser scanning microscope (Carl Zeiss, LSM 710) with excitation at $488 \mathrm{~nm}$ laser and emission at $493 \mathrm{~nm} \sim 634 \mathrm{~nm}$.

FITC-labeled Gap 26 (FITC-C6-VCYDKSFPISHVR, MW=2,053.3) and unlabeled Gap 26 (VCYDKSFPISHVR, MW=1550.8) were acquired from Sigma. Similar to the loading of FITC and FITC-Dextran, Gap 26 was loaded into blank PEGDA microneedles through the incubation in the reservoir for 22 h. FITC conjugated Gap 26 loaded microneedles were imaged by confocal laser scanning microscope (Carl Zeiss, LSM 710) with excitation at $488 \mathrm{~nm}$ laser and emission at $493 \mathrm{~nm} \sim 634 \mathrm{~nm}$. The loading efficiency was calculated by comparing the total amount of peptides and the remaining in solution.

$$
\text { Loading Efficiency }=\frac{\text { Loaded } \operatorname{amount}(\mu \mathrm{g})}{\text { Initial } \operatorname{amount}(\mu \mathrm{g})} \times 100 \%
$$

\subsubsection{Release profile of CPT and Gap26 from microneedles}

Microneedle patches loaded with drugs were immersed in DI water at $37{ }^{\circ} \mathrm{C}$ on a shaking device with a speed of $500 \mathrm{rpm}$. At desired time points, $50 \mu \mathrm{L}$ of solution was transferred to Greiner 96 well plate and the fluorescence intensity was examined with Synergy H4 (CPT: excitation $365 \mathrm{~nm} /$ emission $428 \mathrm{~nm}$; FITC-Gap 26: excitation $495 \mathrm{~nm} /$ emission $525 \mathrm{~nm}$ ). The concentrations were calculated based on standard curves and cumulative released amount of drugs were calculated for different time points.

\subsubsection{Cell viability test by AlamarBlue ${ }^{\circledR}$ assay}

Keloid fibroblasts were seeded in 96 well plate at a density of 1,500 cells per well. After overnight culturing, culture medium was replaced with new culture medium containing different concentrations of free CPT $(0.1 \mu \mathrm{M}, 10 \mu \mathrm{M})$ for $24 \mathrm{~h}$. After $24 \mathrm{~h}$, the medium was removed and cells were gently washed by 
PBS for 3 times. Finally, cell medium with Alamar Blue reagent was added. 4 hours later, fluorescence of the solution (emission/excitation: 555/585 nm) was examined and analyzed according to manufacturer's protocol. All the data was normalized by taking the viability of untreated cells as $100 \%$.

\subsubsection{FRAP assay}

Keloid fibroblasts were seeded at 24 well plate at a density of 40,000 cells per well. Once they reached confluence, cells were incubated with serum-free medium for starvation for $1 \mathrm{~h}$. Then medium was replaced with serum-free medium containing different concentrations of Gap $26(0.125 \mathrm{mg} / \mathrm{mL}, 0.25$ $\mathrm{mg} / \mathrm{mL}, 0.5 \mathrm{mg} / \mathrm{mL}$ ) for $2 \mathrm{~h}$. Later, cells were stained with calcein-AM for 20 min. After washing twice by PBS, cells were imaged through confocal laser scanning microscope (Carl Zeiss, LSM 710) with excitation at $488 \mathrm{~nm}$ and emission at $493 \mathrm{~nm} \sim 616 \mathrm{~nm}$. Thereafter, FRAP assay was performed at a selected specific region with $50 \%$ of $488 \mathrm{~nm}$ laser intensity to bleach the fluorescence for 3 cycles and immediately continuous imaged for $20 \mathrm{~min}$ to record the fluorescence recovery (imaged once per minute).

\subsubsection{Real-time Polymerase Chain Reactions for collagen I expression}

Keloid fibroblasts were seeded at the 24 well plate at a density of 40,000 cells per well. Once they reached confluence, culture medium was replaced with culture medium with different concentrations of free CPT $(0.1 \mu \mathrm{M}, 10 \mu \mathrm{M})$ for $24 \mathrm{~h}$. Later cells were washed by PBS for 3 times before being lysed in Lysis buffer with $\beta$-mercaptoethanol. Subsequently, RNA was extracted by RNAeasy mini kit and the concentration was detected by NanoDrop UV-vis Spectrophotometer. The complementary DNA was synthesized according to the templates of RNA by Bioline SensiFAST cDNA Synthesis Kit. Finally, in order to quantify the gene expression of samples, Real-time Polymerase Chain Reactions (RT-PCRs) were conducted with SYBR Green PCR Master Mix kit by Step One Plus Real-Time PCR System. The process includes denaturation at $95{ }^{\circ} \mathrm{C}$ for $10 \mathrm{~min}$, and amplification for 40 cycles with $95{ }^{\circ} \mathrm{C}$ denaturation for 
$15 \mathrm{~s}$ and $60^{\circ} \mathrm{C}$ extension for $1 \mathrm{~min}$. The forward and backward primer sequences of GAPDH (glyceraldehyde 3-phosphate dehydrogenase) and collagen I are listed in Table 5. The mRNA expression level of collagen I was normalized to the housekeeping gene GAPDH, and then calculated as fold change according to comparative CT method using the $2^{-\Delta \Delta \mathrm{Ct}}$ formula.

Table 5. Primer sequences for real-time polymerase chain reactions (This table is reprinted with copyright from[168])

\begin{tabular}{|c|c|c|}
\hline Gene & Forward primer sequence $\left(5^{\prime}-3^{\prime}\right)$ & Reverse primer sequence (5'-3') \\
\hline GAPDH & ACAACTTTGGTATCGTGGAAGG & GCCATCACGCCACAGTTTC \\
\hline Collagen I & CAGAACGGCCTCAGGTACCA & CAGATCACGTCATCGCACAAC \\
\hline
\end{tabular}

\subsubsection{D agarose hydrogel model}

400,000 keloid fibroblasts were suspended in $75 \mu \mathrm{L}$ complete medium. Then 75 $\mu \mathrm{L}$ of $3 \%$ agarose pre-heated for melting and cooled to $37{ }^{\circ} \mathrm{C}$ was mixed with the cell suspension, in which the bubble formation was avoided. Subsequently, $10 \mu \mathrm{L}$ of the suspension was added to each well of the 48 well plate. After being placed at $4{ }^{\circ} \mathrm{C}$ for $4 \mathrm{~min}$, the agarose gel formed and was placed in the incubator overnight. Later, CPT loaded PEGDA microneedles were inserted into agarose beads for $24 \mathrm{~h}$. After the removal of microneedles, cells were stained with FDA for $20 \mathrm{~min}$ and imaged with confocal microscope (LSM 710).

\subsubsection{Ex-vivo keloid model}

Full-thickness human skin derived from operations to remove excess tissue was purchased from Cell Research Corporation (Singapore). The skin samples were cut to $1.3 \mathrm{~cm} \times 1.3 \mathrm{~cm}$ and maintained in complete medium (DMEM containing $10 \%$ FBS). During the maintenance in the incubator, the epidermis layer was exposed to the air while the dermis was submerged in the medium. In order to identify the location and distribution of injected cells, keloid fibroblasts were stained with DiI ( $1 \mathrm{~mL}$ complete medium with $3 \mu \mathrm{L}$ DiI labeling solution) for 2 h, followed by washing with PBS. Following overnight incubation, DiI-labelled 
cells were trypsinized before resuspension in $100 \mu \mathrm{L}$ cooled medium $(15,000$ cells per $\mu \mathrm{L}$ ). The cell suspension was mixed with $100 \mu \mathrm{L}$ GelTrex matrigel gently, to avoid the generation of bubbles. Thereafter, the cells - matrigel mixture was injected from the dermis-side of the skin sample using a $30 \mathrm{G}$ needle. After resting at room temperature for $15 \mathrm{~min}, 200 \mu \mathrm{L}$ of medium was added and the samples were incubated at $37{ }^{\circ} \mathrm{C}$ overnight. After $24 \mathrm{~h}$, the skin samples were fixed in formalin for $72 \mathrm{~h}$, dehydrated by $30 \%$ sucrose for $8 \mathrm{~h}$, and $4 \%$ sucrose for $18 \mathrm{~h}$. Finally, the samples were frozen in Tissue Freezing Medium and cryosectioned at a thickness of $10 \mu \mathrm{m}$ by Leica CryoStat CM1950. The frozen slides were stored at $-20{ }^{\circ} \mathrm{C}$ before further characterization.

The therapeutic efficacy of drug-loaded microneedles was examined with the skin samples injected with unlabeled keloid fibroblasts. The CPT and FITC loaded PEGDA microneedles were pressed into the skin sample by an applicator (Micropoint Pte Ltd). There were four groups: "CPT MN twice" (CPT MNs (low loading concentration: $18 \mathrm{nmol}$ ) were applied at day 14 and 21), "Gap 26 MN twice" (Gap 26 MNs (loading amount: $125 \mu \mathrm{g}$ ) were applied at day 14 and 21), "CPT MN once” (CPT MNs (low loading concentration: $18 \mathrm{nmol}$ ) were applied once at day 21), and "Gap $26 \mathrm{MN}$ once" (Gap $26 \mathrm{MNs}$ (loading amount: $125 \mu \mathrm{g}$ ) were applied once at day 21). At day 28, the patches were removed from all samples. The skin was fixed in formalin and processed as above for cryosection and immunostaining for collagen I examination.

\subsubsection{Immunostaining for collagen I}

The sliced skin samples were treated by $0.2 \%$ Triton-X 100 in PBS for $10 \mathrm{~min}$, followed by $0.1 \%$ Triton-X 100 in PBS for 5 min. After being washed with PBS 3 times, they were placed in the 1\% BSA in PBS for $30 \mathrm{~min}$ and labeled with primary antibody solution (mouse anti human collagen I, 1 to 100 ) at $4{ }^{\circ} \mathrm{C}$ overnight. Later, the slides were washed by $0.1 \%$ Triton-X 100 in PBS for 3 times and stained with secondary antibody (Goat anti mouse IgG, Rhodamine conjugate, 1 to 100) at room temperature for $4 \mathrm{~h}$. Finally, Hoechst $(10 \mu \mathrm{g} / \mathrm{mL})$ 
was added to stain the nuclei. The stained slides were examined by confocal microscope (LSM 710) (Rhodamine: excitation at $561 \mathrm{~nm}$ and emission at 570 $\mathrm{nm}$ to $660 \mathrm{~nm}$; Hoechst: excitation at $405 \mathrm{~nm}$ and emission at $410 \mathrm{~nm}$ to 543 nm.)

\subsubsection{Statistical analysis}

All values were expressed as means \pm standard deviation. One-way ANOVA and Tukey HSD test was used for statistical analysis with $\mathrm{p}<0.05(*), \mathrm{p}<0.01(* *)$, and $\mathrm{p}<0.001(* * *)$ as significant.

\subsection{Results}

\subsubsection{PEGDA microneedles loaded with hydrophobic camptothecin}

$10 \mu \mathrm{M}$ CPT was shown to inhibit the proliferation of keloid fibroblasts (Figure 30A) and suppress the collagen I expression (Figure 30B). Even only after 24 hours, the proliferative activity of CPT of fibroblasts was reduced by $18.21 \%$. Simultaneously, the cellular collagen I expression per cell decreased $\sim 55 \%$ under the treatment.
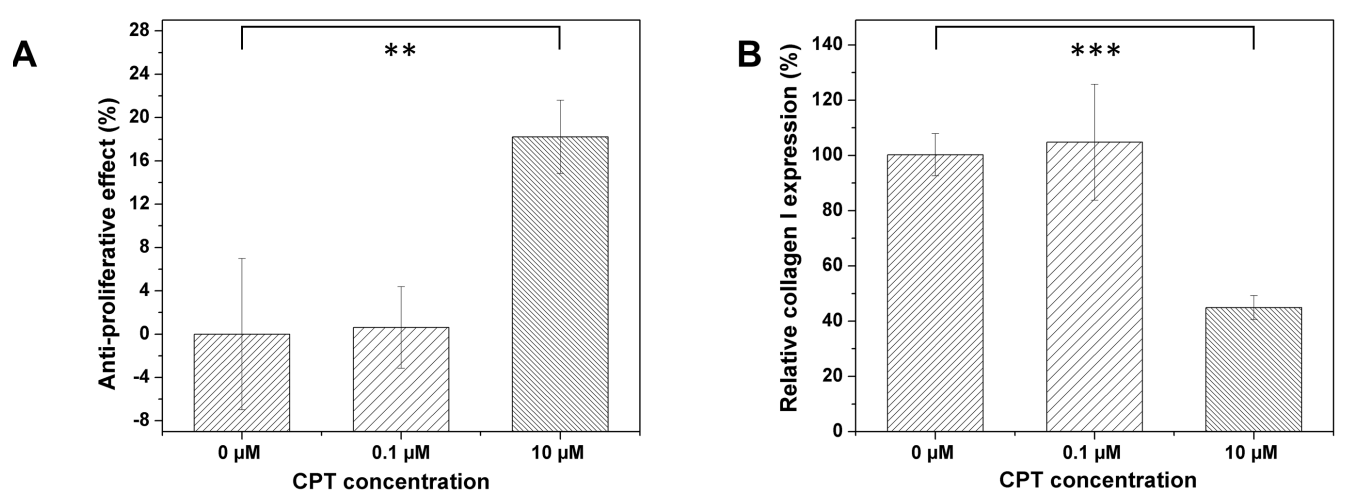

Figure 30. The inhibition of (A) the proliferation and (B) collagen I expression of keloid fibroblasts by camptothecin (CPT). ${ }^{* *} \mathrm{p}<0.01,{ }^{* * *} \mathrm{p}<0.001$. (This figure is reprinted with copyright from[168])

As a hydrophobic molecule, CPT can be loaded into PEGDA microneedles during microneedle fabrication by dissolving it with PEGDA monomer. The PEGDA microneedles exhibited homogeneous pyramid-like structures with a 
base diameter of $300 \mu \mathrm{m}$ and a depth of $\sim 850 \mu \mathrm{m}$ following photopolymerization (Figure 31A, B). The fluorescence from microneedles confirmed the presence of CPT (Figure 32A). The release profile of CPT was determined at desired time points by measuring fluorescence intensity and calculating the released amount based on a standard curve of pre-determined drug concentrations (Figure 32B). Within 24 hours, $0.37 \mathrm{nmol}$ CPT was demonstrated to be released. In 3D agarose gels containing keloid fibroblasts, CPT-loaded microneedles similarly inhibited fibroblast proliferation (Figure 32C, D). Specifically, CPT loaded microneedles treated groups reduced cell proliferation by $30.1 \%$ compared to untreated cells in 24 hours.
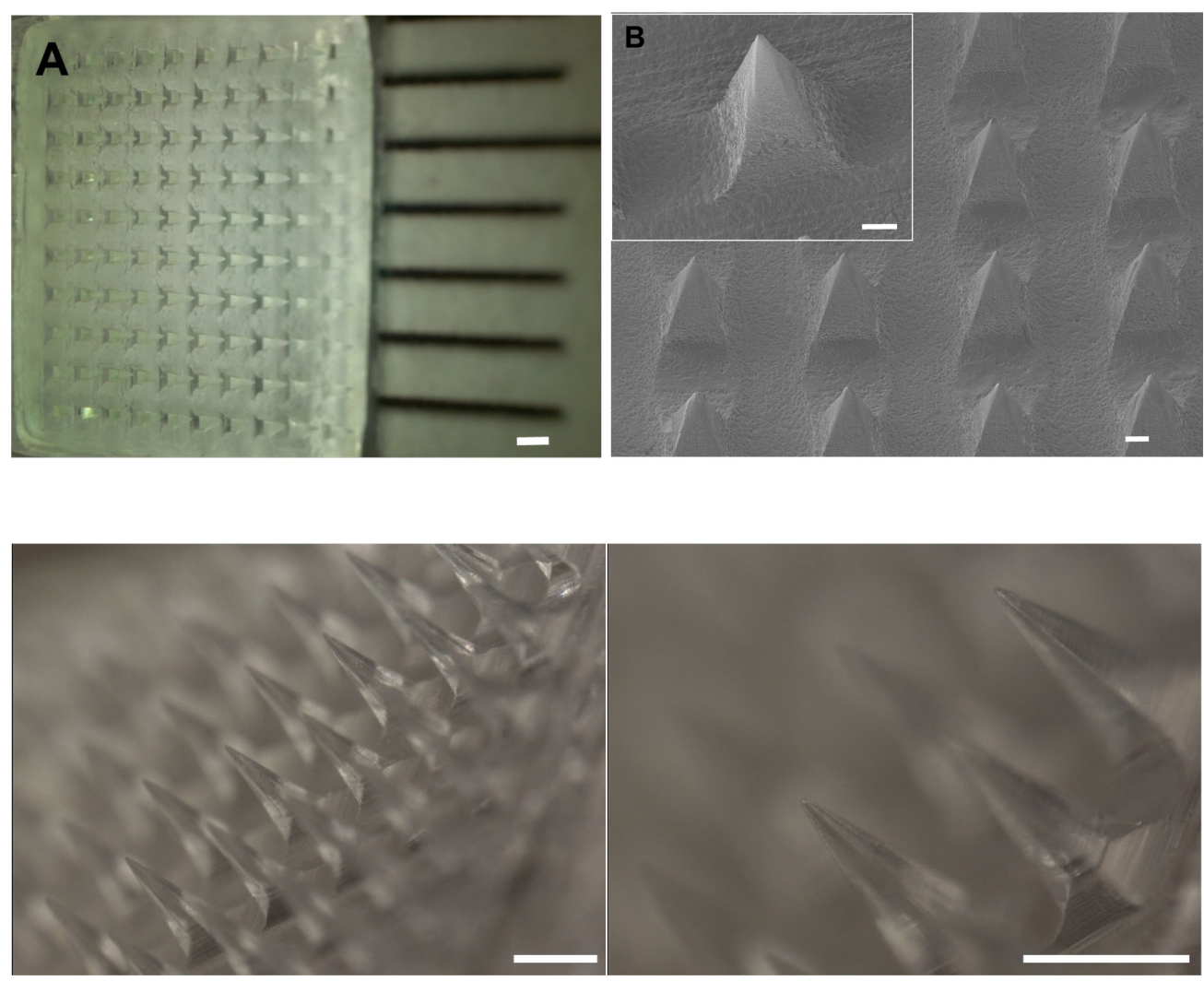

Figure 31. (A) Stereomicroscope images of blank PEGDA microneedles, scale bar: $500 \mu \mathrm{m}$. (B) Scanning electron microscopy (SEM) images of blank PEGDA microneedles, scale bar: $100 \mu \mathrm{m}$. (This figure is reprinted with copyright from[168]) 


\subsubsection{Swelling effect of PEGDA microneedles in aqueous solution}

PEGDA exhibits swelling behavior in aqueous solutions[169]. As shown in Figure 33, PEGDA microneedles fabricated using different UV exposure exhibited different swelling properties. Generally, longer UV exposures resulted in smaller swelling ratios (Q). Microneedles were observed to increase their volume by up to $18.88 \%$. Mesh size can be calculated using the equations 1-4. A higher swelling ratio suggests a larger mesh size (Figure 34). Specifically, the mesh size was $2.0 \mathrm{~nm}$ when the patch was prepared with a short UV exposure time (18s). PEGDA microneedle patch crosslinked with UV exposure for a period of $18 \mathrm{~s}$ was selected for the subsequent experiments.

A

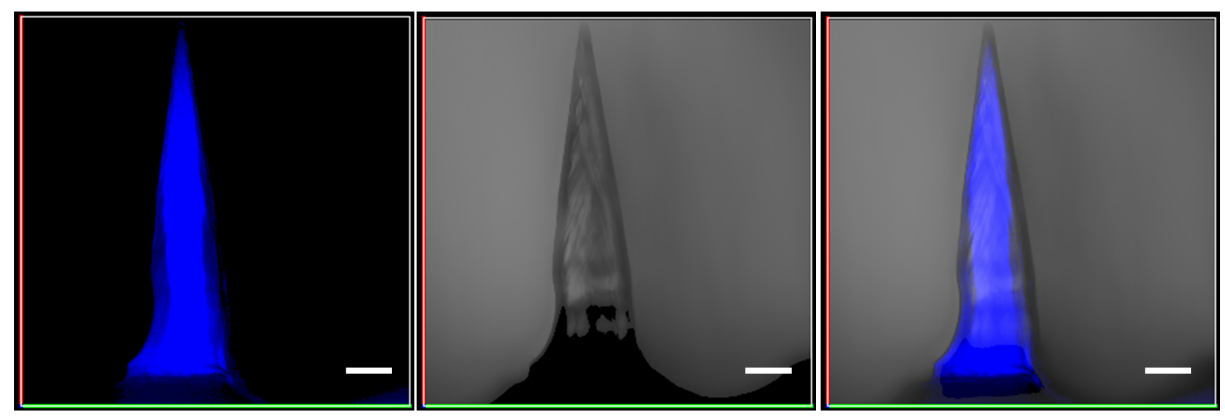

B

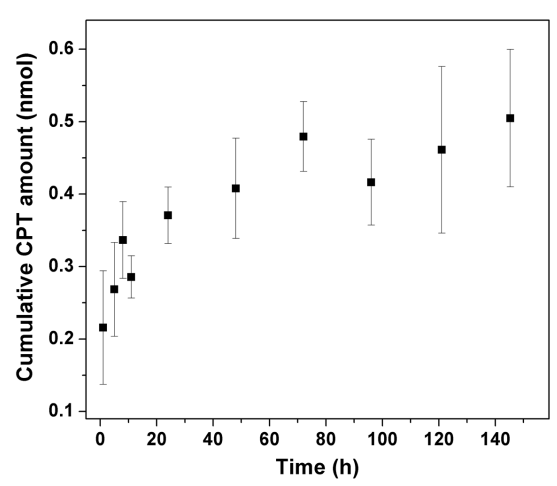

D

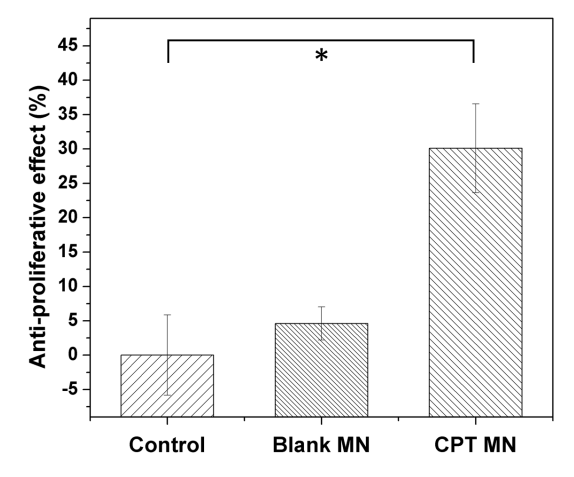

C

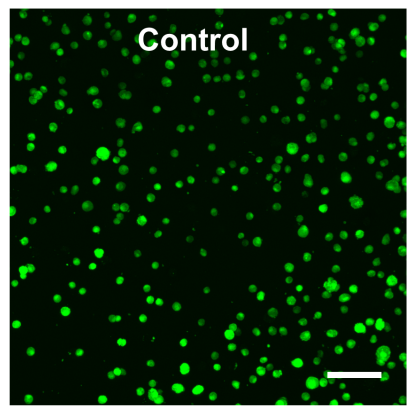

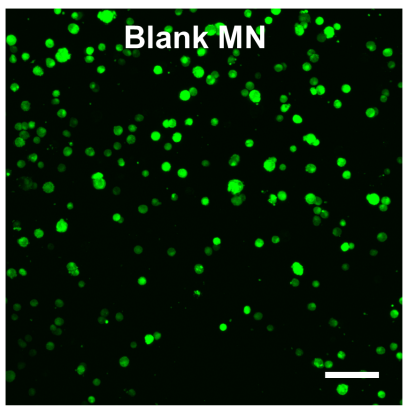

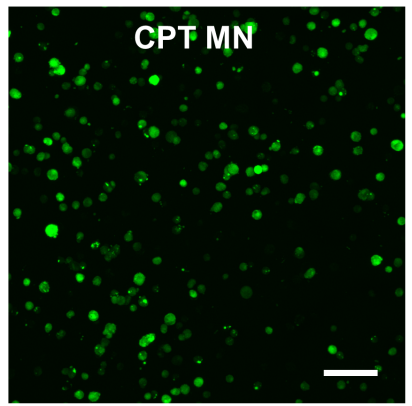


Figure 32. (A) Confocal images of CPT loaded microneedles. Scale bar: $100 \mu \mathrm{m}$. (B) Release profile of CPT from the microneedle patch in PBS. (C) Confocal images of agarose hydrogel containing keloid fibroblasts after the treatment with PEGDA microneedle patches for $24 \mathrm{~h}$, scale bar: $100 \mu \mathrm{m}$. (D) Anti-proliferative effect of CPT loaded microneedles against keloid fibroblasts encapsulated in agarose gel; $* \mathrm{p}<0.05$. (This figure is reprinted with copyright from[168])
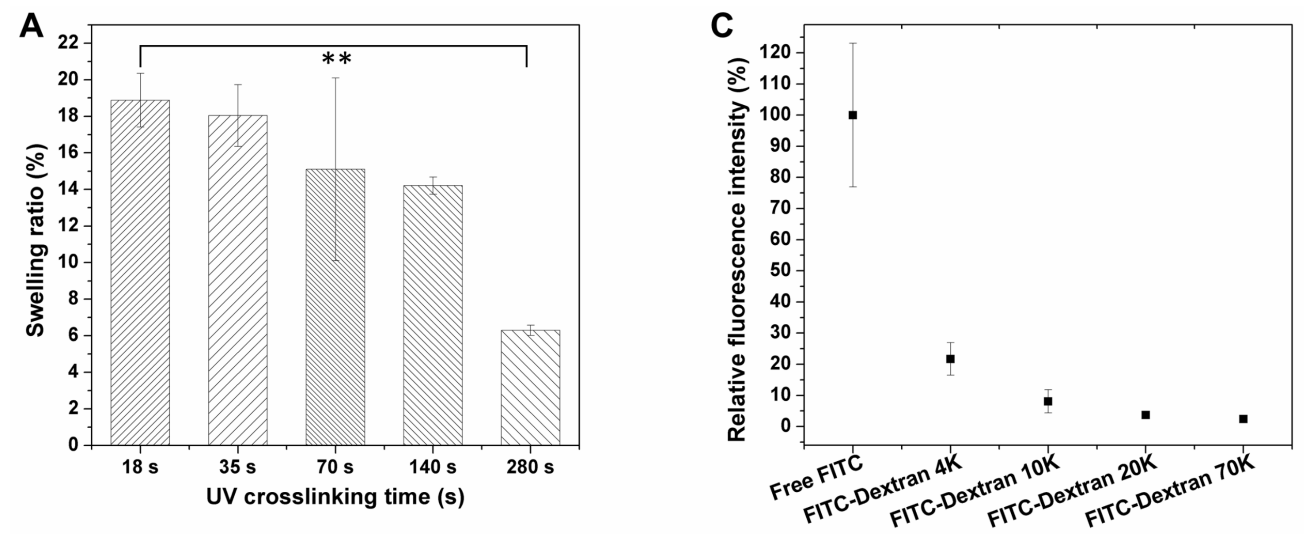

B
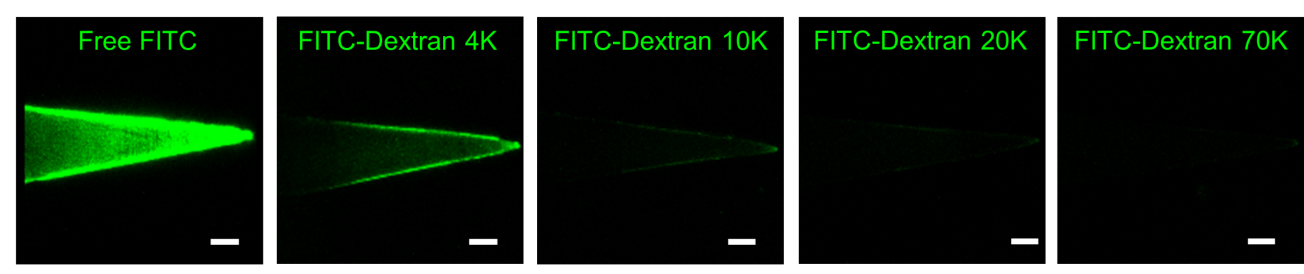

Figure 33. Swell performance of PEGDA microneedles and loading molecules with different molecular weight. (A) Swelling ratio of PEGDA microneedles prepared with different UV exposure time. ${ }^{* *} p<0.01$. (B) Confocal images of PEGDA microneedles loaded with FITC and FITC-Dextran with different molecular weight. Scale bar: $100 \mu \mathrm{m}$. (C) Quantified microneedle fluorescence intensity in B relative to that with free FITC. (This figure is reprinted with copyright from[168])

In the swelling process, molecules like FITC and FITC-Dextran can enter the polymer matrix through diffusion (Figure 33). Obviously, this process is sizedependent, with smaller molecules enter the polymer matrix more readily. In addition, the distribution of loaded FITC and FITC-Dextran molecules within microneedles was inhomogeneous, with more observed at the edge. Increased 
cargo size ( $4 \mathrm{kDa}$ to $70 \mathrm{kDa})$, led to a reduction in loading efficiency as reflected in a decrease in microneedle fluorescence signal (Figure 33B, C). Above 4kDa, the molecules exhibit poor diffusion into the polymeric matrix. Based on the total fluorescence signal from microneedle samples, their relative loading efficiency (compared to free FITC) was found to be $21.7 \%, 8.1 \%, 3.7 \%$, and $2.4 \%$ for FITC-Dextran $4 \mathrm{kDa}, 10 \mathrm{kDa}, 20 \mathrm{kDa}$, and $70 \mathrm{kDa}$, respectively.

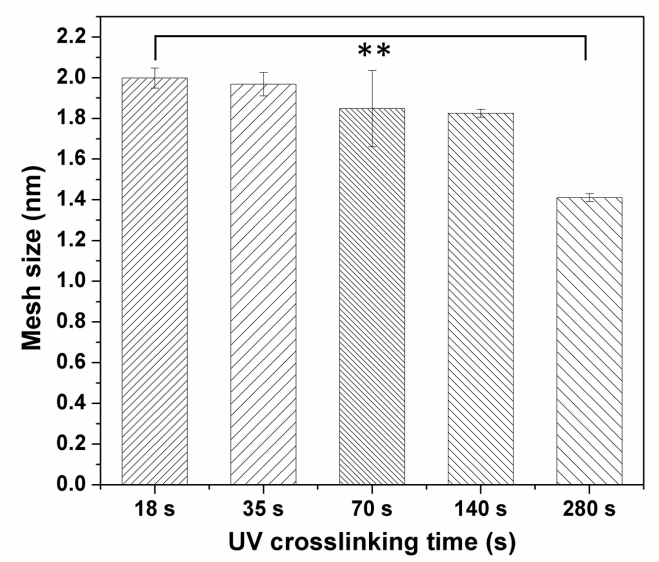

Figure 34. Mesh size of PEGDA microneedles prepared with different UV exposure time. ** $\mathrm{p}<0.01$. (This figure is reprinted with copyright from[168])

\subsubsection{Peptide loaded PEGDA microneedles}

Since the characterization of PEGDA swelling showed that hydrophilic molecules smaller than $4 \mathrm{kDa}$ can be loaded into microneedles, we hypothesized that the peptide Gap 26 (MW=1550.8) could similarly be loaded. Gap26, as a representative connexin mimetic peptide, can mimic specific amino acid sequences of two well-conserved extracellular loops of connexins and interfere with their functions. Gap junctions are formed by intercellular channels composed of connexin proteins, which are known to impact inflammatory response, would closure, and scar formation[80-84]. Suppressing connexins activity by antisense oligodeoxynucleotide was found to reduce the spread of tissue damage, accelerate wound closure, and reduce scarring[85]. So, we hypothesized Gap 26 is able to disturb connexins functions, interfere with gap 
junction based intercellular communications, thus exhibit potential for keloid scar treatment.

Fluorescence recovery after bleach (FRAP) assay was performed to characterize the inhibitory effect of Gap 26 in gap junction based intercellular communication[170]. Fibroblasts were initially incubated with Gap26 before calcein staining. Subsequently, selected area of the cells was photobleached using high power laser (the red box of Figure 35A) and the recovery of calcein fluorescence at that region was recorded (Figure 35B). The fluorescent intensity during the recovery period was compared with the intensity immediately after bleaching. In $20 \mathrm{~min}$, the fluorescence of untreated cells increased to 5.89 folds. However, the presence of Gap 26 peptide significantly delayed this recovery. For example, only a 1.56-fold increase in the fluorescence intensity was observed in keloid fibroblasts treated with $0.5 \mathrm{mg} / \mathrm{mL}$ Gap 26. This suggests considerable attenuation of intercellular communication by gap junctions, indicating the efficacy of Gap26 against connexins, intercellular channels, showing promise for the improvement of scar treatment.

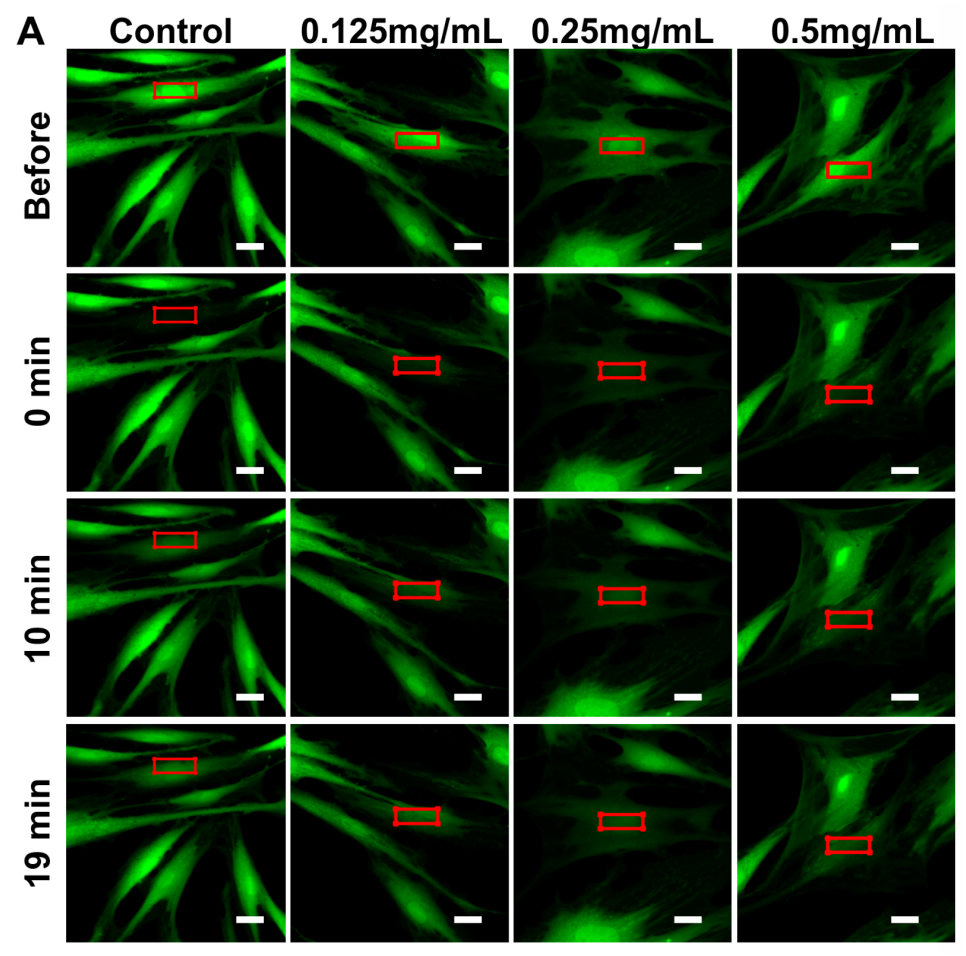




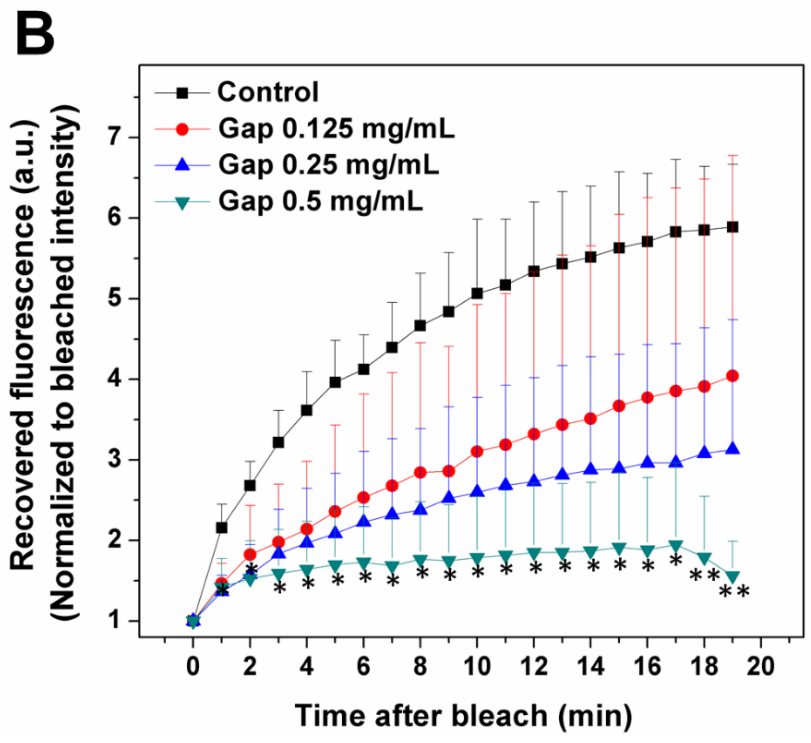

C
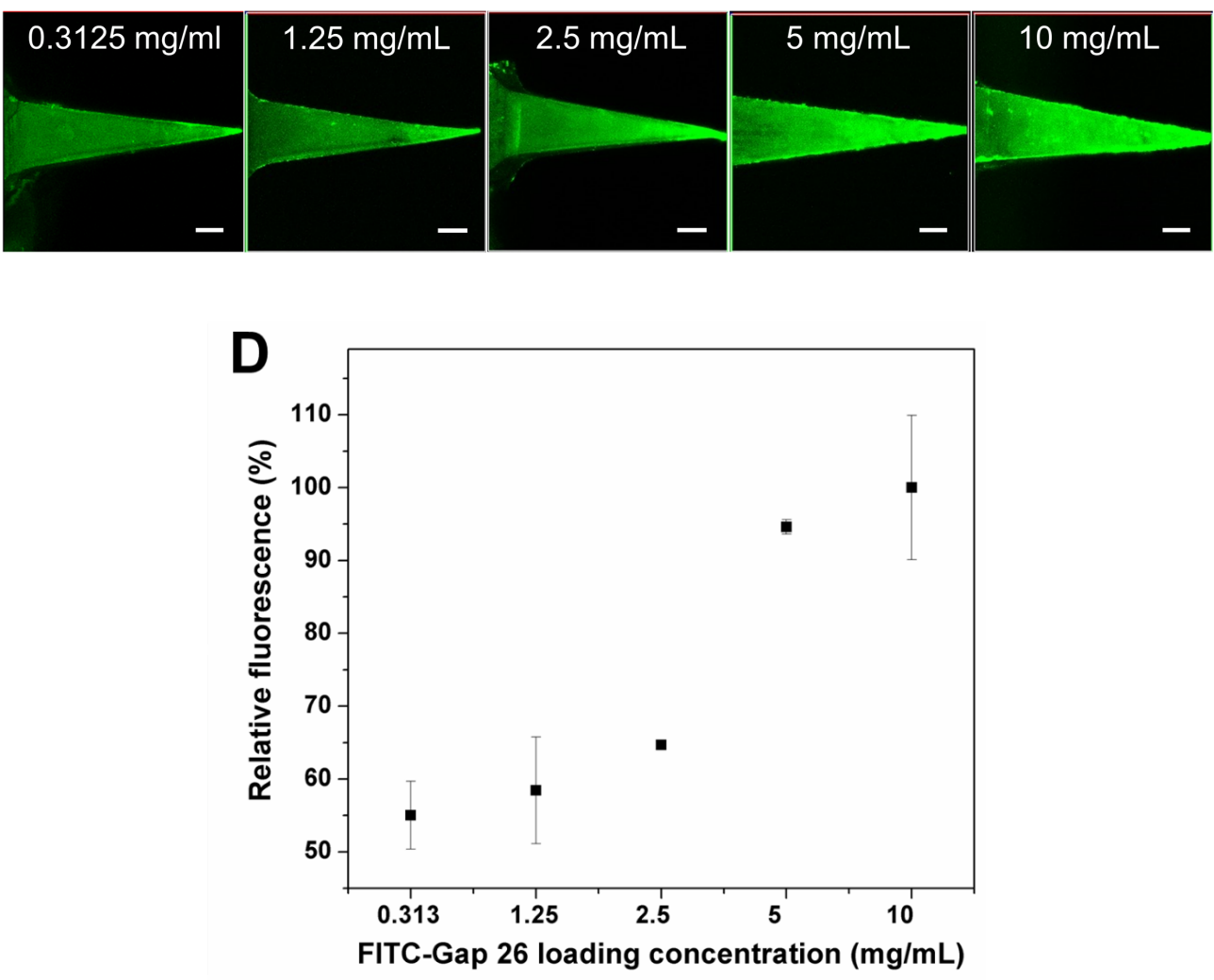
Figure 35. Effect of Gap 26 on FRAP of keloid fibroblasts and loading of FITC-Gap 26 into PEGDA microneedles. (A) Confocal images of keloid fibroblasts in FRAP experiment with the treatment of Gap 26. Scale bar: $20 \mu \mathrm{m}$. (B) Recovered fluorescence intensity change during fluorescence recovery. $* p<0.05, * * p<0.01$. (C) Confocal images of Gap26 loaded PEGDA microneedles. Scale bar: $100 \mu \mathrm{m}$. (D) Quantitative analysis of C. (This figure is reprinted with copyright from[168])

Subsequently, we loaded PEGDA microneedles with Gap26 through the swelling effect of PEGDA. Gap26 was labeled with FITC to facilitate quantification. As shown in Figure 35C, D, a higher peptide concentration in the incubation process provided a higher peptide loading in the microneedles. By quantifying the peptides in the loading solution before and after incubation, the loading efficiency of peptide was calculated (Table 6). For example, $252.13 \pm 5.94 \mu \mathrm{g}$ of Gap26 can be loaded to one patch of microneedles (10 by 10 tips) with $50.43 \% \pm 1.19 \%$ loading efficiency for the initial loading amount of $500 \mu \mathrm{g}$. As the loading process was performed with only the microneedle tips immersed inside the hydrophilic solution, the drugs were only localized in the tips without excess drug trapped in the base. This maximizes the utilization of the drugs. In this example, each tip was loaded with $2.52 \pm 0.06 \mu \mathrm{g}$ of Gap26.

Table 6. FITC-Gap 26 loading amount and efficiency into PEGDA microneedles by swelling effect. (This table is reprinted with copyright from[168])

\begin{tabular}{|c|c|c|c|c|}
\hline Initial amount in solution $(\mu \mathrm{g})$ & $\mathbf{5 0 0}$ & $\mathbf{2 5 0}$ & $\mathbf{1 2 5}$ & $\mathbf{6 2 . 5}$ \\
\hline Initial concentration in solution $(\mu \mathrm{g} / \mu \mathrm{L})$ & 5 & 2.5 & 1.25 & 0.625 \\
\hline Loaded amount $(\mu \mathrm{g})$ & $252.13 \pm 5.94$ & $130.99 \pm 15.13$ & $51.75 \pm 19.85$ & $27.81 \pm 1.40$ \\
\hline Loading efficiency $(\%)$ & $50.43 \pm 1.19$ & $52.40 \pm 6.05$ & $41.40 \pm 15.88$ & $44.50 \pm 2.25$ \\
\hline
\end{tabular}

The loaded peptides were promptly released once the microneedles were placed in the aqueous solution. As shown in Figure 36, 13.47 $\pm 4.52 \mu \mathrm{g}$ of FITC-Gap 26 can be released from the tips of microneedles that contained $252.13 \pm 5.94 \mu \mathrm{g}$ peptides in total. 


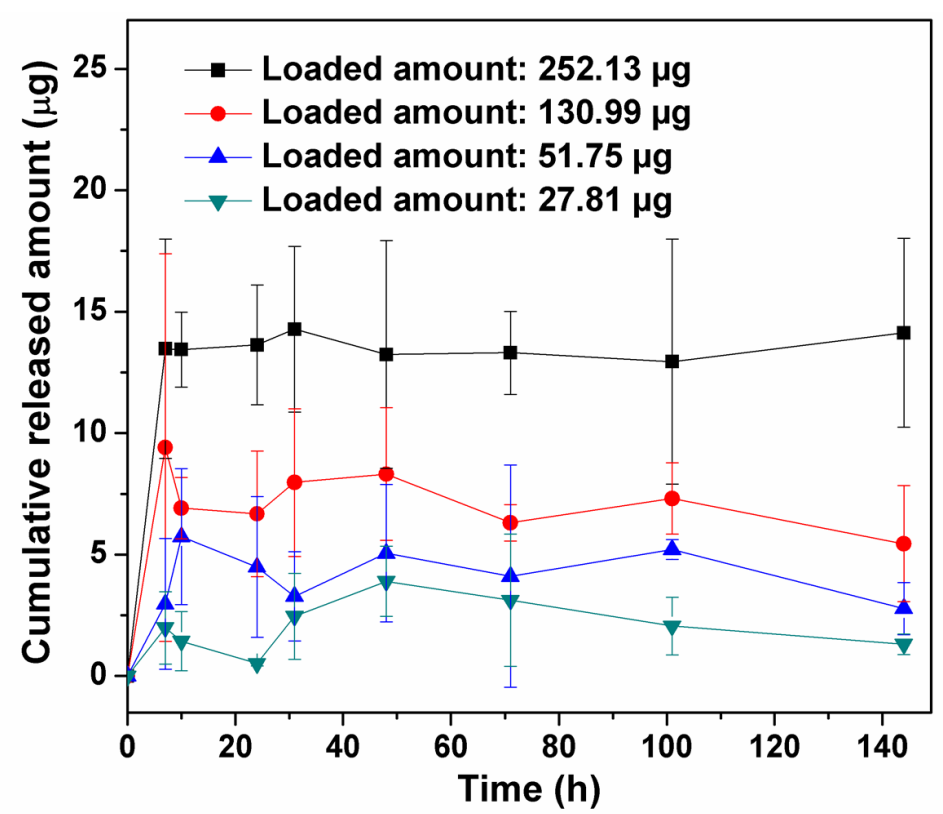

Figure 36. Cumulative release of Gap26 from the microneedle patch. (This figure is reprinted with copyright from[168])

\subsubsection{Therapeutic effect of Gap26 loaded microneedles in ex-vivo keloid model}

A keloid disease model was next used to explore the potential efficacy of Gap26-loaded microneedles. Briefly, the model was built by injecting keloid fibroblasts into the dermis region of an ex vivo human skin sample (Figure 37A). Keloid fibroblasts were labeled with the lipophilic dye DiI (Figure 38) to enable identification (Figure 37B).

Before the study commenced, keloid fibroblasts were injected into the dermis region of the skin. At day 14, the microneedle patch was applied on the skin for one week. The patch was then replaced with a new one at day 21 for another week to sustain drug release and maintain drug amount within the skin. We studied two types of microneedle patches, CPT- and Gap26- loaded microneedles. At day 28, skin samples were fixed and sectioned to examine the collagen I expression. 

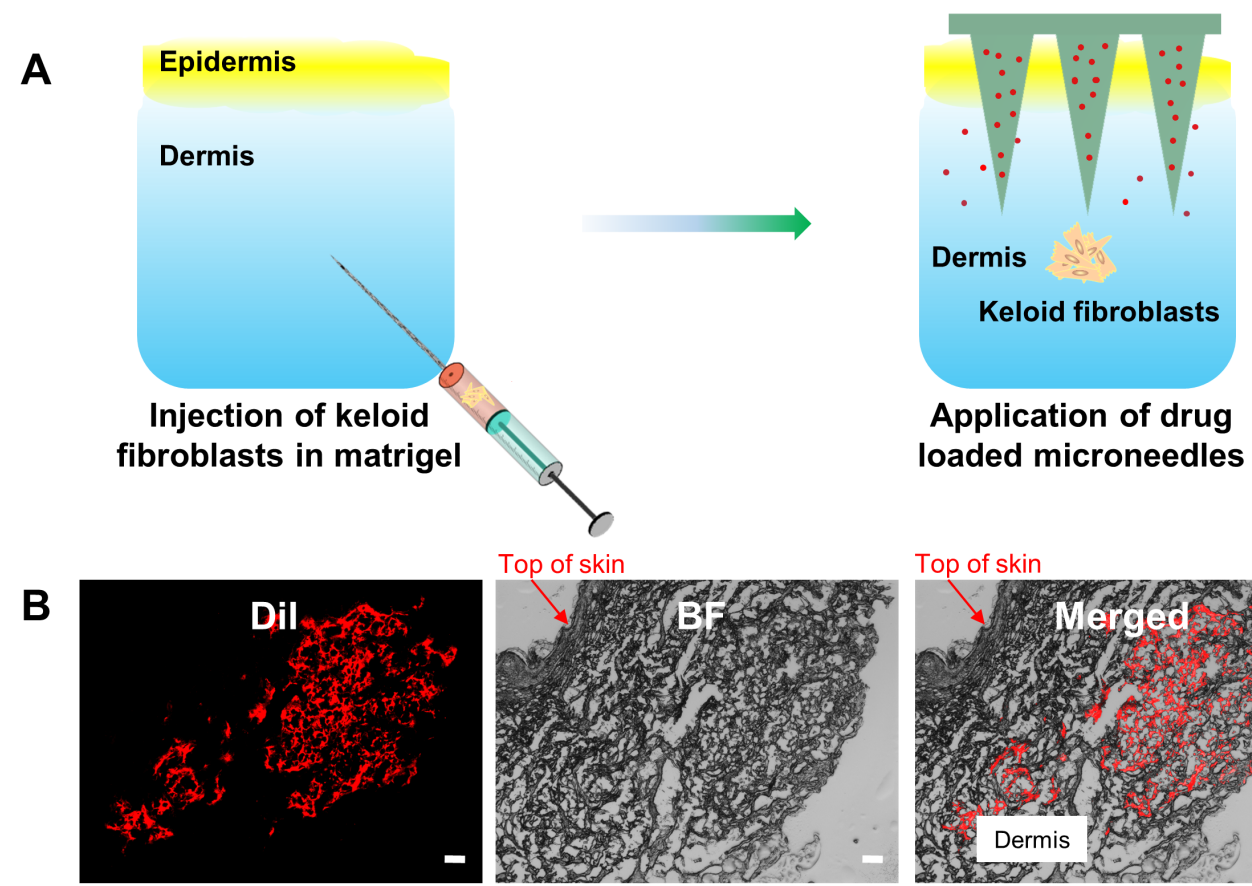

Figure 37. Ex-vivo keloid scar model and injection of keloid fibroblasts. (A) Illustration of the ex-vivo keloid model. (B) Presence of DiI labelled keloid fibroblasts in the dermis region of skin samples. Scale bar: $100 \mu \mathrm{m}$. (This figure is reprinted with copyright from[168])
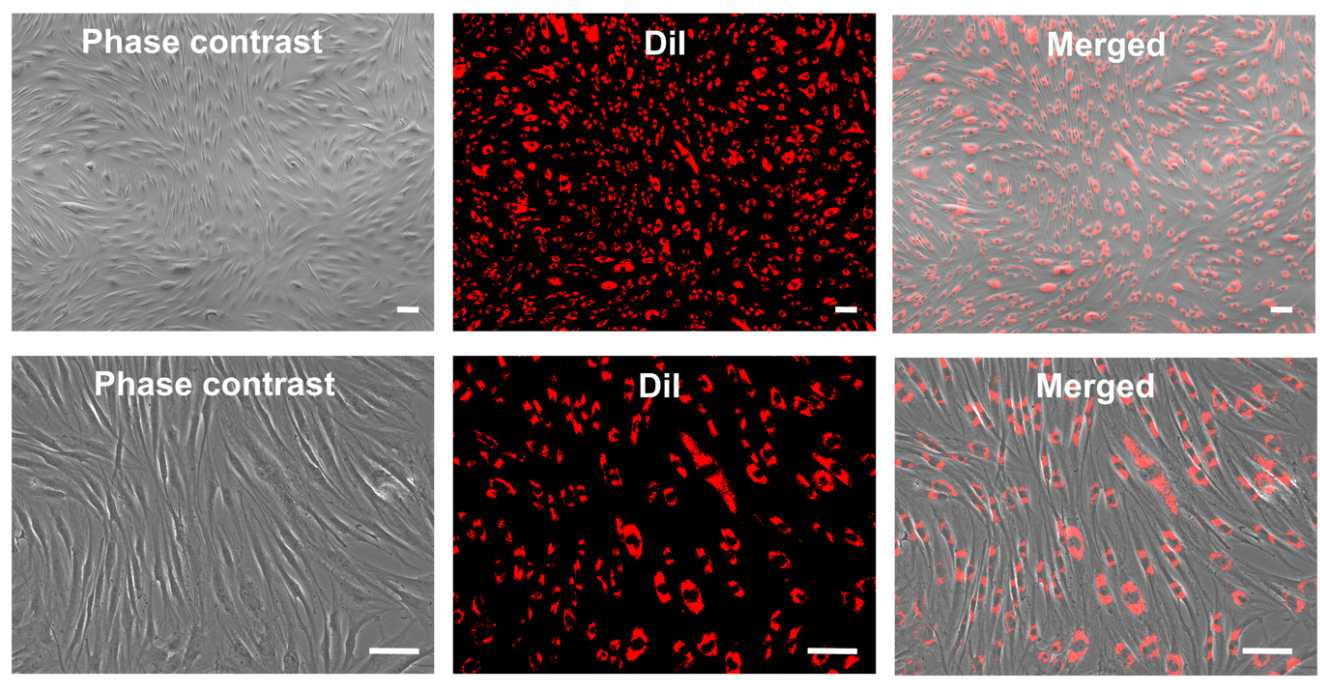

Figure 38. Keloid fibroblasts labelled with DiI. scale bar: $100 \mu \mathrm{m}$. (This figure is reprinted with copyright from[168]) 
The presence of keloid fibroblasts increased the expression of collagen I in the dermis layer of the skin (Figure 39A), which is reminiscent of native keloid scars $[165,171]$.

Keloid scar is characterized by abundant deposition of collagen, especially collagen type I[172]. The treatment with Gap 26 loaded microneedles twice (“Gap $26 \mathrm{MN}$ twice”) reduced the collagen I expression to 36.9\% (Figure 39B). Treatment with CPT loaded microneedles twice ("CPT MN twice") also suppressed collagen I expression to $47.3 \%$ (Figure 40 ). The data reflected a promising trend although there is no significant difference $(p>0.05)$, probably due to the individualized difference and limited number of skin samples and more samples can be tested in the further studies. The PEGDA microneedles still maintained their homogeneous pyramid tips structure after application in the skin samples and didn't dissolve or deform in the skin to leave any remnants as shown in Figure 41. These results indicated the CPT and Gap26-loaded microneedles were effective to inhibit the collagen I expression from keloid fibroblasts, demonstrating their potential efficacy for scar treatment.

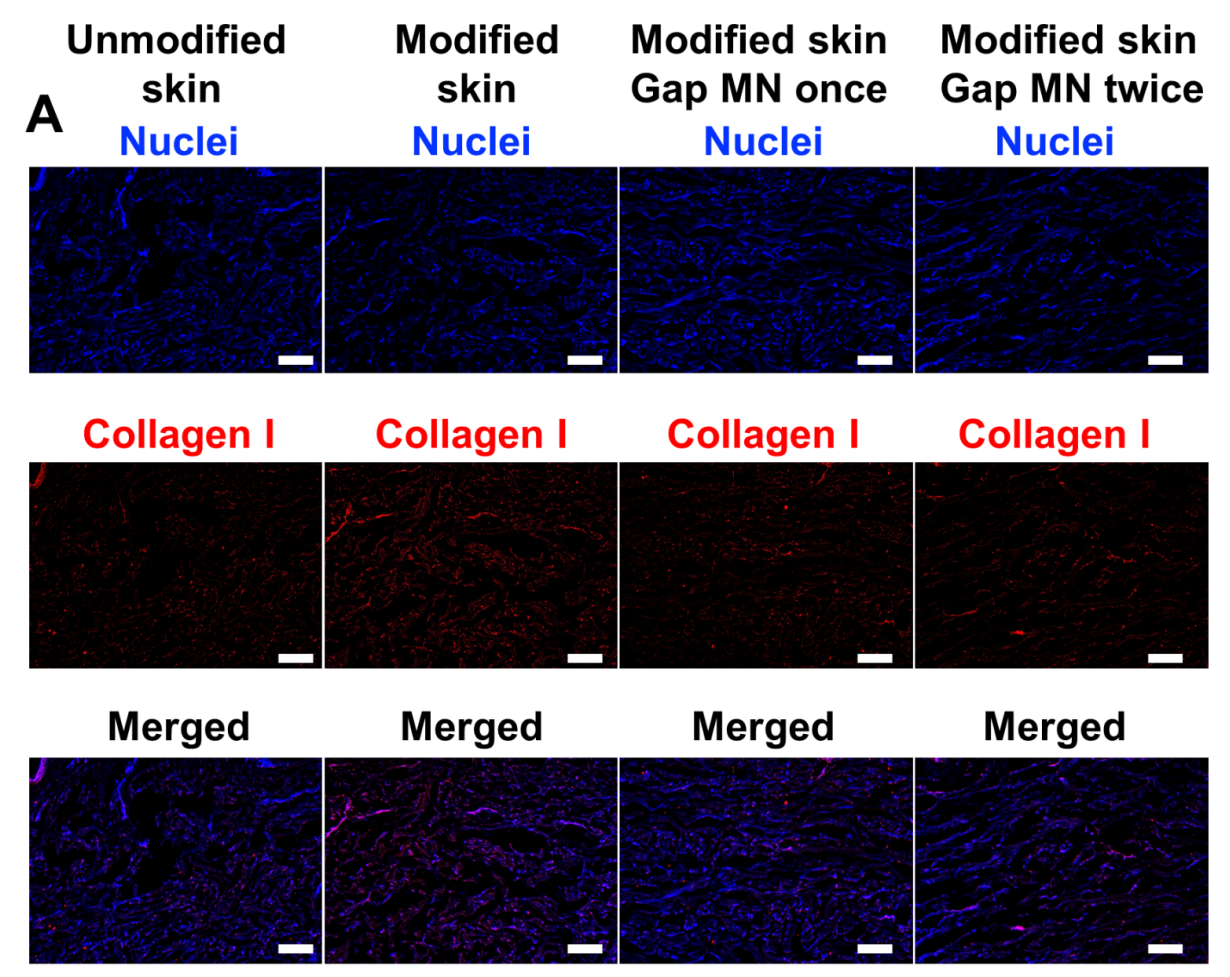




\section{B}

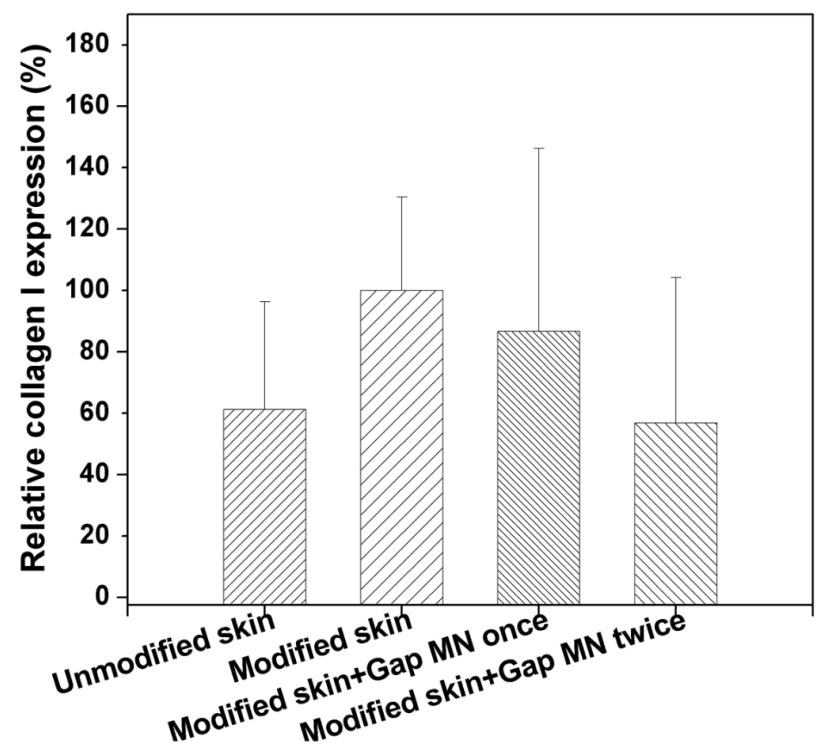

Figure 39. Effect of Gap 26 loaded microneedles on collagen I expression of skin samples in the ex vivo model. (A) Immunostaining of Collagen I expression after the treatment with Gap26loaded microneedles. Scale bar: $100 \mu \mathrm{m}$. (B) Quantitative analysis of collagen I expressions in A by normalization to the modified skin without treatment. (This figure is reprinted with copyright from[168])

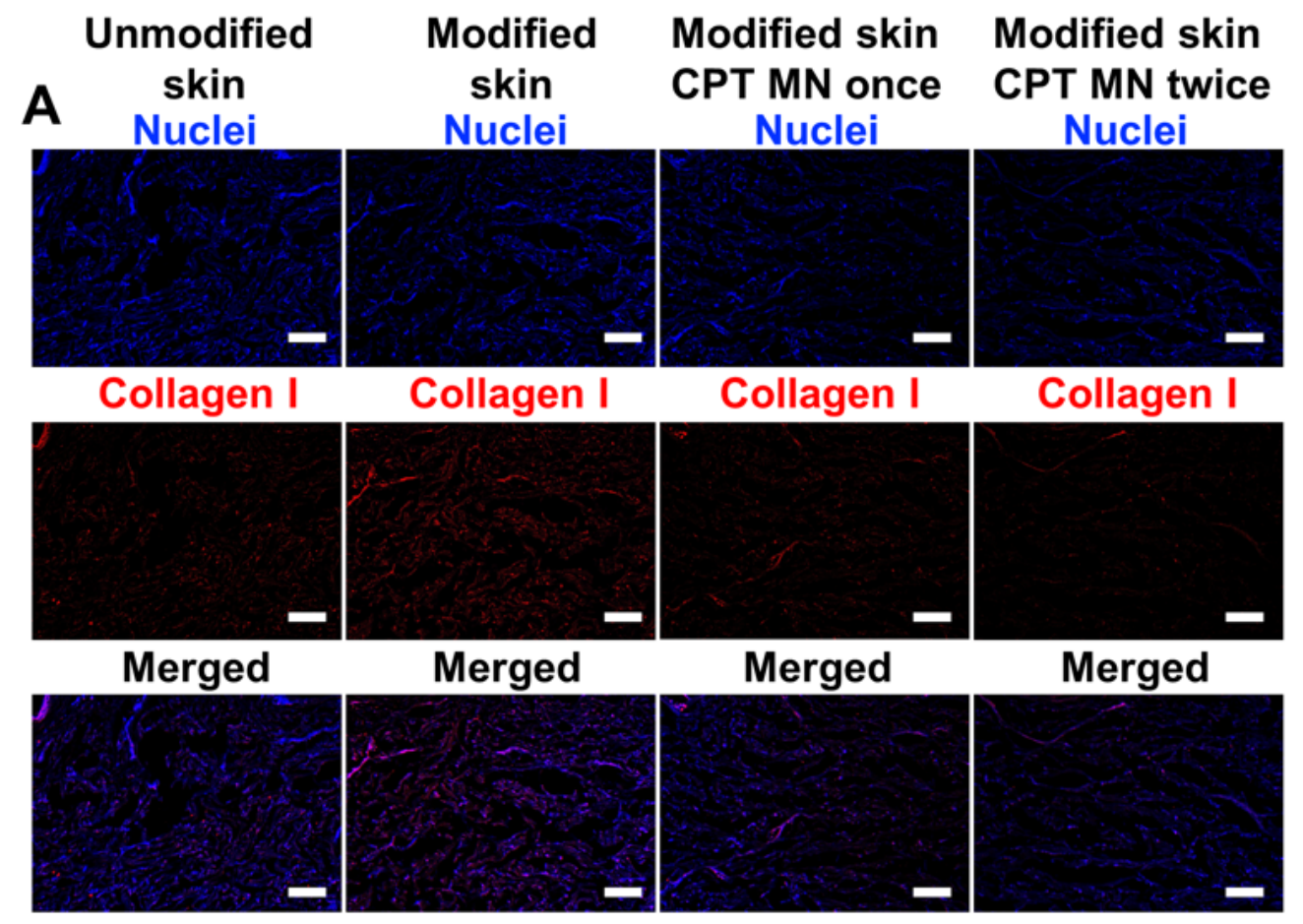




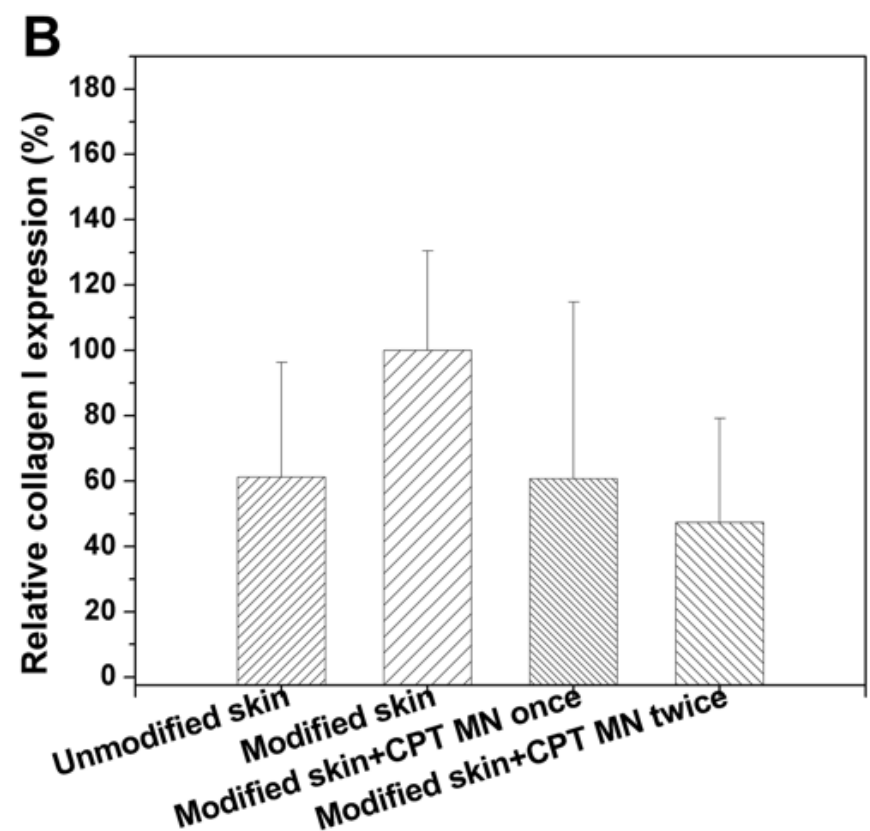

Figure 40. (A) Immunostaining of Collagen I expression in the ex vivo keloid model after the treatment with CPT loaded microneedles. Scale bar: $100 \mu \mathrm{m}$. (B) Quantitative analysis of collagen I expressions in A by normalization to the keloid model without treatment. (This figure is reprinted with copyright from[168])
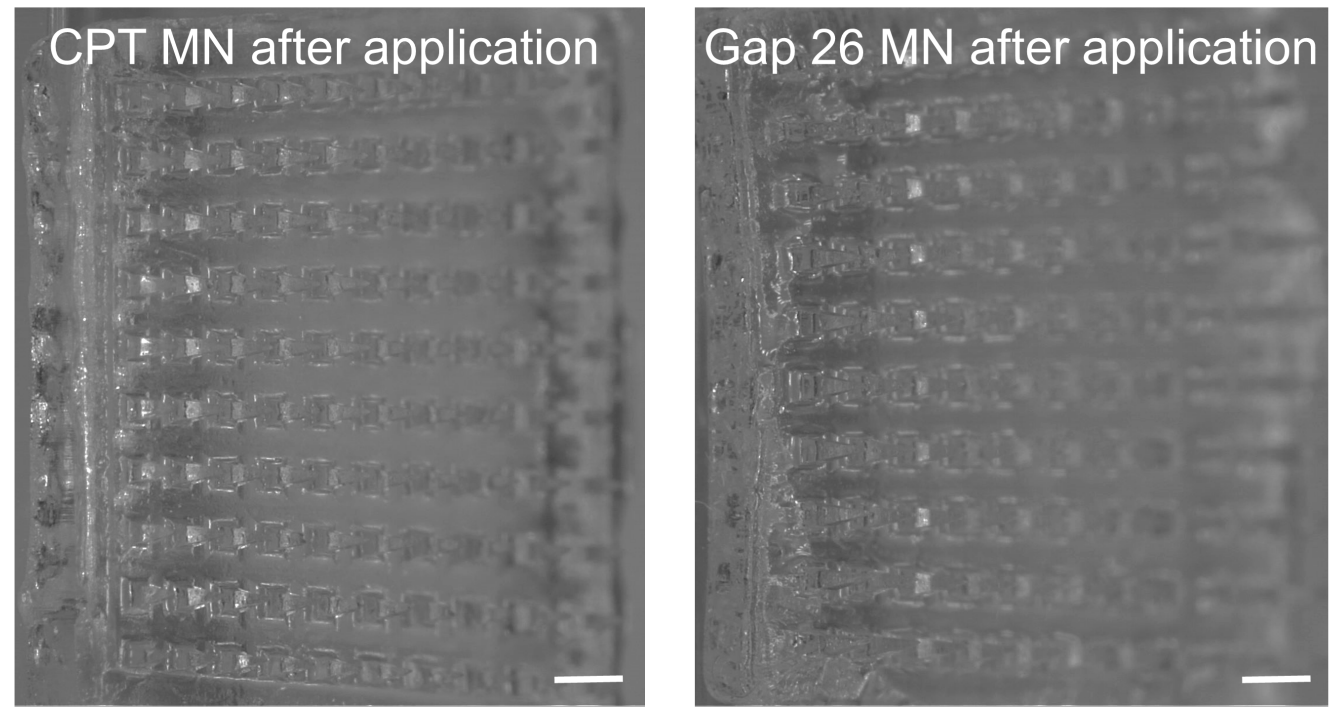

Figure 41. Stereomicroscope images of CPT and Gap26 loaded PEGDA microneedles after application in the ex vivo skin samples, scale bar: $500 \mu \mathrm{m}$. (This figure is reprinted with copyright from[168]) 


\subsection{Discussion}

In recent years, there has been increasing interest to apply biomolecules including peptide/protein as targeted therapeutics. Delivery these molecules by microneedles can avoid their digestions by gastrointestinal enzymes during oral delivery. Previously reported methods to load these biomolecules in the microneedles have been limited. In this paper, we developed a facile, effective, and gentle strategy to load peptide into biocompatible PEGDA microneedles. A model peptide, Gap26 (connexin mimetic peptide) was chosen as a candidate therapeutic and applied on an ex vivo keloid scar model to prove this concept.

To date, PEGDA microneedles have mainly been used to deliver small molecular drugs (e.g. 5-fluororacil (5-FU) and curcumin) that are chemically stable to high temperature, light, and extreme $\mathrm{pH}$ conditions. These stable molecules can be mixed with the monomer before UV exposure or heating to generate microneedles. For example, CPT can reduce collagen biosynthesis, a key step in scar formation[35, 43]. When this drug was encapsulated in PEGDA microneedles through photolithography, no noticeable change was observed in its efficacy to inhibit cell proliferation and collagen I expression.

Subsequently, we loaded hydrophilic sugar and peptide molecules by swelling PEGDA microneedles in an aqueous solution. Upon hydration, the PEGDA matrix allows solutes to diffuse into the hydrogel matrix. As discussed above, the mesh size of PEG hydrogel[173, 174], related to its crosslinking density determines whether molecules can readily diffuse into the matrix. Thus, molecules larger than the mesh size cannot be efficiently loaded into PEGDA. Here, we controlled the mesh size of PEGDA microneedles by tuning the UV exposure time during the fabrication and ended up with a PEGDA matrix with a mesh size of $2.0 \mathrm{~nm}$. Thereafter, we tested the loading of various molecules with distinct molecular weights (FITC, FITC-dextran 4kDa, 10kDa, 20kDa). Due to the difference in molecular weight, their hydrodynamic sizes vary from angstrom scale to $1.3 \mathrm{~nm}$ to $2.0 \mathrm{~nm}$ to $2.8 \mathrm{~nm}$ [157]. In this case, FITC-dextran 
molecules bigger than 10kDa are larger than the mesh size of PEGDA. Thus, it is expected that minimal quantities of larger molecules were observed in microneedles. Furthermore, Stokes-Einstein equation indicates diffusion is related with the radius of the solute on a fixed temperature and solvent $[175$, 176]. So, it is reasonable to observe higher signals for the needles loaded with FITC than FITC-dextran 4kDa.

Next, we chose the connexin mimetic peptide, Gap $26(\mathrm{MW}=1550.8)$ as a model drug to be loaded into PEGDA microneedles and applied for keloid scar therapy. Inhibition of cell-cell communications through gap junctional couplings is an emerging concept to treat scar[84]. Connexin mimetic peptides like Gap 26 are valuable tools to inhibit connexin functions, making it promising for scar reduction[87, 177]. We confirmed Gap26 can suppress the cell-cell communication between keloid fibroblasts and loaded Gap 26 into PEGDA microneedles. The loading of Gap26 is similar to that of dextran. As indicated in Fick's law, $J=-D \nabla \varphi$, where $\mathrm{J}$ is the diffusion flux of the amount per unit area per unit time, $\mathrm{D}$ is the diffusion coefficient, $\nabla \varphi$ is the concentration gradient. The peptide solution with higher concentration generates bigger concentration gradient, which allows more peptide molecules to diffuse into the polymeric network given a similar time period and surface area. An interesting phenomenon is that FITC and FITC-dextran $4 \mathrm{kDa}$ were mainly located on the edges of microneedles, while Gap26 exhibited more homogenous distribution after loading. This may be related to their different molecular structures, which requires the detailed further studies. The amount loaded to each tip of microneedles through this strategy can reach $2.52 \pm 0.06 \mu \mathrm{g}$ of Gap26, which is similar to the previously reported coating method[93]. However, our loading strategy is comparatively more straightforward since formulation viscosity or surface tension does not need adjustment.

After $7 \mathrm{~h}, 13.47 \pm 4.52 \mu \mathrm{g}$ of FITC-Gap 26 was released from microneedles with $252.13 \pm 5.94 \mu \mathrm{g}$ loaded. As the release was detected by immersing the whole 
patch inside water at $37^{\circ} \mathrm{C}$, the swelling occurs throughout the whole patch. As such, released Gap26 from the tips may enter the matrix at the base of microneedles as well, possibly resulting in the relatively low proportion of Gap 26 release. Nonetheless, this situation is not likely to occur in the future therapeutic applications, as skin penetration is limited to microneedle tips.

The efficacy of Gap26-loaded microneedles for keloid scar treatment was investigated on an ex vivo keloid scar model. Application of Gap 26-loaded microneedles (especially when applied twice in the treatment period; 4 weeks) could inhibit collagen I expression efficiently, demonstrating the possibility of this peptide loading system in the treatment of keloid scar. This signifies an interesting transdermal drug delivery system, which is potentially for loading other small molecular weight hydrophilic drug molecules in the applications of various diseases.

\subsection{Conclusions}

In summary, a facile, effective, and gentle strategy is reported to load peptides into PEGDA microneedles by exploiting the swelling phenomena of PEGDA in aqueous solutions. By regulating UV crosslinking time, the swelling ratio and mesh size of PEGDA microneedles can be controlled. This allows loading of molecules less than $4 \mathrm{kDa}$. As a proof of concept, the model peptide, Gap 26 was loaded into the PEGDA microneedles to suppress the gap junction based intercellular communication between keloid fibroblasts which leads to reduced collagen I expression in an ex vivo model. This exhibits its therapeutic potentials for keloid scar.

(This chapter is reprinted with copyright from[168]) 


\section{Chapter 5: Conclusions and Future Work}

\subsection{Conclusions}

In this thesis, two problems existed in regenerative medicine are solved including long-term tracking mesenchymal stem cell differentiation by biocompatible and highly photostable fluorescent nanoparticles and keloid scar treatment by transdermal delivering connexin mimetic peptide through PEGDA microneedles.

First of all, a new type of highly fluorescent and bioresorbable polymeric NPs were prepared through nanoprecipitation from a well-defined polymer, PCLDPP-PCL, in the presence of Pluronic ${ }^{\circledR} 127$ as the stabilizer. Covalent insertion of DPP as a highly fluorescent moiety into the middle of each PCL chain and generated steric effect effectively suppress the $\pi-\pi$ aggregation of DPP in the PCL matrix to reduce the bleaching effect. The NPs prepared were highly fluorescent and could be internalized by MSCs with little cytotoxicity. Most importantly, the PCL-DPP-PCL NPs exhibited significantly enhanced photostability compared to several commercial organic fluorophores, the physically blended DPPHT/PCL NPs and Qtracker® for imaging stem cells.

Secondly, these PCL-DPP-PCL nanoparticles were applied for long-term tracking of mesenchymal stem cell differentiation. In vitro cell experiments exhibited the nanoparticles labelling did not compromise the cell viability and demonstrated different performances on the multilineage potency of the MSCs during differentiation into three lineages. Specifically, compared to the unlabeled control cells, this probe labeling compromised MSC osteogenic differentiation, but didn't show any negative effects for adipogenic and chondrogenic as verified by gene expressions and histological staining. Furthermore, these fluorescent probes were able to maintain their strong fluorescence intensity even after 4 weeks of differentiation. This study 
demonstrated that PCL-DPPPCL nanoparticles could be used for long-term cell tracking in MSC differentiation into adipogenic and chondrogenic lineages.

Finally, regarding to the keloid scar treatment issue in the regenerative medicine field, we introduced a facile, effective, and gentle strategy to load PEGDA microneedles with hydrophilic peptide, such as connexin mimetic peptides (Gap26) without compromising peptide potency through the swelling effect of PEGDA in the aqueous solution. By regulating UV crosslinking time, the swelling ratio, and mesh size of PEGDA microneedles can be controlled. Gap 26 was loaded into the PEGDA microneedles to suppress the gap junction-based intercellular communication between keloid fibroblasts which leads to reduced collagen I expression in an ex vivo model. This exhibits its therapeutic potentials for keloid scar.

\subsection{Limitations and future work}

Up to now, this thesis has managed to develop a biocompatible and photostable fluorescent probe, which demonstrated superb photostability compared to organic dyes, could maintain fluorescent signals at a higher ratio compared to commercial quantum dots, and exhibited much potentials for long-term tracking MSCs' adipogenic and chondrogenic differentiation (up to 4 weeks) without disturbing their viability, proliferation, and differentiation performance. However, they showed negative effects on the osteogenic differentiation of MSCs with reducing the representative mRNA expressions. The detailed reason is still unknown based on the current results and requires further studies, but the different influences of NPs on each specific lineage differentiation indicated it's necessary to investigate their effects individually before the final application.

On the other hand, the biosafety and degradation studies should be performed to investigate in vivo before their real application in vivo. Besides, the current fluorophore-DPP has limitations for the long-term tracking applications in vivo since its emission is still in the visible range. Recent decades witnessed the 
advanced development of near infrared (NIR) dyes, which are more attractive for in vivo imaging with two main advantages. Firstly, NIR light is able to penetrate deeper into tissue compared to the visible light, which allows to interpret deeper tissue structure information. Secondly, NIR dye can provide better signal to noise ratio because there is less autofluorescence in the NIR range compared to visible light. So the future work can apply this strategy to enhance photostability of NIR dye for in vivo studies.

Chapter 4 reported a facile and non-disruptive strategy to load hydrophilic peptide into polymeric microneedles and demonstrated effects for keloid scar treatment. This system can be also used for loading other hydrophilic molecules, such as sugar, siRNA by the swelling of microneedles. However, this delivery system is currently only for the hydrophilic molecules with molecular weight less than $4 \mathrm{kDa}$. Loading bigger molecules above this threshold will be achieved by further optimizations and controls to increase the swelling ratio of the microneedles but maintain the mechanical strength to penetrate the skin.

In addition, an ex vivo keloid scar model was developed in this thesis to test the efficacy of drug loaded microneedles. However, the effect is still not satisfying enough for the clinical applications. In the future, to obtain better effects, combination of two or more drugs can be explored. This thesis has demonstrated both hydrophobic and hydrophilic drugs can be loaded inside the PEGDA microneedles. The synergistic effect of two or more drugs have much possibility to demonstrate better results for the treatment of keloids. In addition, a wellrecognized animal model of keloid scar should be established although with difficulties for testing all kinds of transdermal delivery systems. 


\section{Reference}

[1] A.S. Mao, D.J. Mooney, Regenerative medicine: Current therapies and future directions, Proc Natl Acad Sci U S A. 2015, 112, 14452-9.

[2] T.J. Nelson, A. Behfar, S. Yamada, A. Martinez-Fernandez, A. Terzic, Stem cell platforms for regenerative medicine, Clin Transl Sci. 2009, 2, 222-7.

[3] V. Tabar, L. Studer, Pluripotent stem cells in regenerative medicine: challenges and recent progress, Nat Rev Genet. 2014, 15, 82-92.

[4] B. Bose, K.R. Katikireddy, P.S. Shenoy, Regenerative medicine for diabetes: differentiation of human pluripotent stem cells into functional betacells in vitro and their proposed journey to clinical translation, Vitam Horm. 2014, 95, 223-48.

[5] S.D. Schwartz, C.D. Regillo, B.L. Lam, D. Eliott, P.J. Rosenfeld, N.Z. Gregori, J.P. Hubschman, J.L. Davis, G. Heilwell, M. Spirn, J. Maguire, R. Gay, J. Bateman, R.M. Ostrick, D. Morris, M. Vincent, E. Anglade, L.V. Del Priore, R. Lanza, Human embryonic stem cell-derived retinal pigment epithelium in patients with age-related macular degeneration and Stargardt's macular dystrophy: follow-up of two open-label phase 1/2 studies, Lancet. 2015, 385, 509-16.

[6] F.W. Pagliuca, J.R. Millman, M. Gurtler, M. Segel, A. Van Dervort, J.H. Ryu, Q.P. Peterson, D. Greiner, D.A. Melton, Generation of functional human pancreatic beta cells in vitro, Cell. 2014, 159, 428-39.

[7] P. Menasche, V. Vanneaux, J.R. Fabreguettes, A. Bel, L. Tosca, S. Garcia, V. Bellamy, Y. Farouz, J. Pouly, O. Damour, M.C. Perier, M. Desnos, A. Hagege, O. Agbulut, P. Bruneval, G. Tachdjian, J.H. Trouvin, J. Larghero, Towards a clinical use of human embryonic stem cell-derived cardiac progenitors: a translational experience, European Heart Journal. 2015, 36, 74350 .

[8] M. Eapen, J.E. Wagner, Transplant Outcomes in Acute Leukemia (I), Semin Hematol. 2010, 47, 46-50.

[9] S. Tipnis, C. Viswanathan, Umbilical cord matrix derived mesenchymal stem cells can change the cord blood transplant scenario, Int J Stem Cells. 2010, 3, 103-18.

[10] K. Takahashi, S. Yamanaka, Induction of pluripotent stem cells from mouse embryonic and adult fibroblast cultures by defined factors, Cell. 2006, 126, 663-76.

[11] K. Takahashi, K. Tanabe, M. Ohnuki, M. Narita, T. Ichisaka, K. Tomoda, S. Yamanaka, Induction of pluripotent stem cells from adult human fibroblasts by defined factors, Cell. 2007, 131, 861-72.

[12] J. Yu, M.A. Vodyanik, K. Smuga-Otto, J. Antosiewicz-Bourget, J.L. Frane, S. Tian, J. Nie, G.A. Jonsdottir, V. Ruotti, R. Stewart, Slukvin, II, J.A. Thomson, Induced pluripotent stem cell lines derived from human somatic cells, Science. 2007, 318, 1917-20. 
[13] I.H. Park, N. Arora, H. Huo, N. Maherali, T. Ahfeldt, A. Shimamura, M.W. Lensch, C. Cowan, K. Hochedlinger, G.Q. Daley, Disease-specific induced pluripotent stem cells, Cell. 2008, 134, 877-86.

[14] M. Csobonyeiova, S. Polak, J. Koller, L. Danisovic, Induced pluripotent stem cells and their implication for regenerative medicine, Cell Tissue Bank. 2015, 16, 171-80.

[15] K.K. Hirschi, S. Li, K. Roy, Induced pluripotent stem cells for regenerative medicine, Annu Rev Biomed Eng. 2014, 16, 277-94.

[16] G.G. Walmsley, J. Hyun, A. McArdle, K. Senarath-Yapa, M.S. Hu, M.T. Chung, V.W. Wong, M.T. Longaker, D.C. Wan, Induced pluripotent stem cells in regenerative medicine and disease modeling, Curr Stem Cell Res Ther. 2014, 9, 73-81.

[17] H. Kamao, M. Mandai, S. Okamoto, N. Sakai, A. Suga, S. Sugita, J. Kiryu, M. Takahashi, Characterization of human induced pluripotent stem cell-derived retinal pigment epithelium cell sheets aiming for clinical application, Stem Cell Reports. 2014, 2, 205-18.

[18] S. Reardon, D. Cyranoski, Japan stem-cell trial stirs envy, Nature. 2014, $513,287-8$.

[19] A.J. Wagers, I.L. Weissman, Plasticity of adult stem cells, Cell. 2004, 116, 639-48.

[20] A. Behfar, A. Terzic, Mesenchymal stem cells: engineering regeneration, Clin Transl Sci. 2008, 1, 34-5.

[21] D.G. Phinney, D.J. Prockop, Concise review: mesenchymal stem/multipotent stromal cells: the state of transdifferentiation and modes of tissue repair--current views, Stem Cells. 2007, 25, 2896-902.

[22] A. Uccelli, L. Moretta, V. Pistoia, Mesenchymal stem cells in health and disease, Nat Rev Immunol. 2008, 8, 726-36.

[23] A.I. Caplan, Adult mesenchymal stem cells for tissue engineering versus regenerative medicine, J Cell Physiol. 2007, 213, 341-7.

[24] K. Le Blanc, O. Ringden, Immunomodulation by mesenchymal stem cells and clinical experience, J Intern Med. 2007, 262, 509-25.

[25] P. Bianco, X. Cao, P.S. Frenette, J.J. Mao, P.G. Robey, P.J. Simmons, C.Y. Wang, The meaning, the sense and the significance: translating the science of mesenchymal stem cells into medicine, Nat Med. 2013, 19, 35-42.

[26] C. Nombela-Arrieta, J. Ritz, L.E. Silberstein, The elusive nature and function of mesenchymal stem cells, Nat Rev Mol Cell Biol. 2011, 12, 126-31. [27] P.K. Nguyen, D. Nag, J.C. Wu, Methods to assess stem cell lineage, fate and function, Adv Drug Deliv Rev. 2010, 62, 1175-86.

[28] C. Xu, L. Mu, I. Roes, D. Miranda-Nieves, M. Nahrendorf, J.A. Ankrum, W. Zhao, J.M. Karp, Nanoparticle-based monitoring of cell therapy, Nanotechnology. 2011, 22, 494001.

[29] C. Xu, W. Zhao, Nanoparticle-based monitoring of stem cell therapy, Theranostics. 2013, 3, 616-7. 
[30] P.A.C. Bhranti S. Shah, Eduardo K. Moioli, Michael A. Stroscio, and, J.J. Mao, Labeling of mesenchymal stem cells by bioconjugated quantum dots, Nano Lett. 2007, 7, 3071-9.

[31] S. Danner, H. Benzin, T. Vollbrandt, J. Oder, A. Richter, C. Kruse, Quantum dots do not alter the differentiation potential of pancreatic stem cells and are distributed randomly among daughter cells, Int J Cell Biol. 2013, 2013, 918242.

[32] R.B. Altman, D.S. Terry, Z. Zhou, Q. Zheng, P. Geggier, R.A. Kolster, Y. Zhao, J.A. Javitch, J.D. Warren, S.C. Blanchard, Cyanine fluorophore derivatives with enhanced photostability, Nat Methods. 2012, 9, 68-71.

[33] R. Hachani, M. Lowdell, M. Birchall, N.T. Thanh, Tracking stem cells in tissue-engineered organs using magnetic nanoparticles, Nanoscale. 2013, 5, 11362-73.

[34] X. Wang, L. Yang, Z.G. Chen, D.M. Shin, Application of nanotechnology in cancer therapy and imaging, CA Cancer J Clin. 2008, 58, 97-110.

[35] A. Vollrath, S. Schubert, U.S. Schubert, Fluorescence imaging of cancer tissue based on metal-free polymeric nanoparticles-a review, J Mater Chem B. 2013, 1, 1994.

[36] R. Naccache, E.M. Rodriguez, N. Bogdan, F. Sanz-Rodriguez, C. Cruz Mdel, A.J. Fuente, F. Vetrone, D. Jaque, J.G. Sole, J.A. Capobianco, High resolution fluorescence imaging of cancers using lanthanide ion-doped upconverting nanocrystals, Cancers (Basel). 2012, 4, 1067-105.

[37] J.M. Lee, B.S. Kim, H. Lee, G.I. Im, In vivo tracking of mesechymal stem cells using fluorescent nanoparticles in an osteochondral repair model, Mol Ther. 2012, 20, 1434-42.

[38] H. Ow, D.R. Larson, M. Srivastava, B.A. Baird, W.W. Webb, U. Wiesner, Bright and stable core-shell fluorescent silica nanoparticles, Nano Lett. 2005, 5, 113-7.

[39] C. Wu, D.T. Chiu, Highly fluorescent semiconducting polymer dots for biology and medicine, Angew Chem Int Ed. 2013, 52, 3086-109.

[40] A. Kaeser, A.P.H.J. Schenning, Fluorescent nanoparticles based on selfassembled $\pi$-conjugated systems, Adv Mater. 2010, 22, 2985-97.

[41] G.T. Dempsey, J.C. Vaughan, K.H. Chen, M. Bates, X. Zhuang, Evaluation of fluorophores for optimal performance in localization-based super-resolution imaging, Nat Methods. 2011, 8, 1027-36.

[42] A. Wagh, S.Y. Qian, B. Law, Development of biocompatible polymeric nanoparticles for in vivo NIR and FRET imaging, Bioconjug Chem. 2012, 23, 981-92.

[43] A. Reisch, P. Didier, L. Richert, S. Oncul, Y. Arntz, Y. Mély, A.S. Klymchenko, Collective fluorescence switching of counterion-assembled dyes in polymer nanoparticles, Nat Commun. 2014, 5, 4089.

[44] Q. Zheng, M.F. Juette, S. Jockusch, M.R. Wasserman, Z. Zhou, R.B. Altman, S.C. Blanchard, Ultra-stable organic fluorophores for single-molecule research, Chem Soc Rev. 2014, 43, 1044-56. 
[45] W. Qin, K. Li, G. Feng, M. Li, Z. Yang, B. Liu, B.Z. Tang, Bright and photostable organic fluorescent dots with aggregation-induced emission characteristics for noninvasive long-term cell imaging, Adv Funct Mater. 2014, 24, 635-43.

[46] I.L. Medintz, H.T. Uyeda, E.R. Goldman, H. Mattoussi, Quantum dot bioconjugates for imaging, labelling and sensing, Nat Mater. 2005, 4, 435-46.

[47] B.A. Kairdolf, A.M. Smith, T.H. Stokes, M.D. Wang, A.N. Young, S. Nie, Semiconductor quantum dots for bioimaging and biodiagnostic applications, Annu Rev Anal Chem (Palo Alto Calif). 2013, 6, 143-62.

[48] P. Wu, X.P. Yan, Doped quantum dots for chemo/biosensing and bioimaging, Chem Soc Rev. 2013, 42, 5489-521.

[49] X. Michalet, F.F. Pinaud, L.A. Bentolila, J.M. Tsay, S. Doose, J.J. Li, G. Sundaresan, A.M. Wu, S.S. Gambhir, S. Weiss, Quantum dots for live cells, in vivo imaging, and diagnostics, Science. 2005, 307, 538-44.

[50] M. Bruchez, M. Moronne, P. Gin, S. Weiss, A.P. Alivisatos, Semiconductor Nanocrystals as Fluorescent Biological Labels, Science. 1998, 281, 2013-6.

[51] A.P. Alivisatos, Semiconductor clusters, nanocrystals, and quantum dots, Science. 1996, 271, 933-7.

[52] P. Zrazhevskiy, M. Sena, X. Gao, Designing multifunctional quantum dots for bioimaging, detection, and drug delivery, Chem Soc Rev. 2010, 39, 432654.

[53] X. Wu, H. Liu, J. Liu, K.N. Haley, J.A. Treadway, J.P. Larson, N. Ge, F. Peale, M.P. Bruchez, Immunofluorescent labeling of cancer marker Her2 and other cellular targets with semiconductor quantum dots, Nat Biotechnol. 2003, 21, 41-6.

[54] H.T.U. Igor L. Medintz, Ellen R. Goldman and Hedi Mattoussi, Quantum dot bioconjugates for imaging, labelling and sensing, Nat Mater. 2005, 4, 43546.

[55] F. Tang, L. Li, D. Chen, Mesoporous silica nanoparticles: synthesis, biocompatibility and drug delivery, Adv Mater. 2012, 24, 1504-34.

[56] Q. Cai, Z.S. Luo, W.Q. Pang, Y.W. Fan, X.H. Chen, F.Z. Cui, Dilute solution routes to various controllable morphologies of MCM-41 silica with a basic medium, Chem Mater. 2001, 13, 258-63.

[57] C.E. Fowler, D. Khushalani, B. Lebeau, S. Mann, Nanoscale materials with mesostructured interiors, Adv Mater. 2001, 13, 649-52.

[58] H. Yamauchi, T. Ishikawa, S. Kondo, Surface characterization of ultramicro spherical-particles of silica prepared by W/O microemulsion method, Colloids Surf. 1989, 37, 71-80.

[59] H. Ow, D.R. Larson, M. Srivastava, B.A. Baird, W.W. Webb, U. Wiesner, Bright and stable core-shell fluorescent silica nanoparticles, Nano Lett. 2005, 5, 113-7.

[60] T.H. Chung, S.H. Wu, M. Yao, C.W. Lu, Y.S. Lin, Y. Hung, C.Y. Mou, Y.C. Chen, D.M. Huang, The effect of surface charge on the uptake and 
biological function of mesoporous silica nanoparticles 3T3-L1 cells and human mesenchymal stem cells, Biomaterials. 2007, 28, 2959-66.

[61] D.M. Huang, T.H. Chung, Y. Hung, F. Lu, S.H. Wu, C.Y. Mou, M. Yao, Y.C. Chen, Internalization of mesoporous silica nanoparticles induces transient but not sufficient osteogenic signals in human mesenchymal stem cells, Toxicol Appl Pharmacol. 2008, 231, 208-15.

[62] X.E. Jiang, J. Dausend, M. Hafner, A. Musyanovych, C. Rocker, K. Landfester, V. Mailander, G.U. Nienhaus, Specific effects of surface amines on polystyrene nanoparticles in their Interactions with mesenchymal sem cells, Biomacromolecules. 2010, 11, 748-53.

[63] A. Tautzenberger, S. Lorenz, L. Kreja, A. Zeller, A. Musyanovych, H. Schrezenmeier, K. Landfester, V. Mailander, A. Ignatius, Effect of functionalised fluorescence-labelled nanoparticles on mesenchymal stem cell differentiation, Biomaterials. 2010, 31, 2064-71.

[64] D.N. Tran, L.C. Ota, J.D. Jacobson, W.C. Patton, P.J. Chan, Influence of nanoparticles on morphological differentiation of mouse embryonic stem cells, Fertil Steril. 2007, 87, 965-70.

[65] Y. Wang, C. Xu, H. Ow, Commercial nanoparticles for stem cell labeling and tracking, Theranostics. 2013, 3, 544-60.

[66] D. Del Toro, R. Dedhia, T.T. Tollefson, Advances in scar management: prevention and management of hypertrophic scars and keloids, Curr Opin Otolaryngol Head Neck Surg. 2016, 24, 322-9.

[67] G. Juckett, H. Hartman-Adams, Management of keloids and hypertrophic scars, Am Fam Physician. 2009, 80, 253-60.

[68] A.E. Brissett, D.A. Sherris, Scar contractures, hypertrophic scars, and keloids, Facial Plast Surg. 2001, 17, 263-72.

[69] B. Berman, O.A. Perez, S. Konda, B.E. Kohut, M.H. Viera, S. Delgado, D. Zell, Q. Li, A review of the biologic effects, clinical efficacy, and safety of silicone elastomer sheeting for hypertrophic and keloid scar treatment and management, Dermatol Surg. 2007, 33, 1291-302.

[70] F.B. Niessen, P.H. Spauwen, J. Schalkwijk, M. Kon, On the nature of hypertrophic scars and keloids: a review, Plast Reconstr Surg. 1999, 104, 143558.

[71] P. Omo-Dare, Genetic studies on keloid, J Natl Med Assoc. 1975, 67, 42832.

[72] J.A. Atkinson, K.T. McKenna, A.G. Barnett, D.J. McGrath, M. Rudd, A randomized, controlled trial to determine the efficacy of paper tape in preventing hypertrophic scar formation in surgical incisions that traverse Langer's skin tension lines, Plast Reconstr Surg. 2005, 116, 1648-56.

[73] M.K. Atkinson JA, Barnett AG, McGrath DJ, Rudd, M., Randomized controlled trial to determine the efficacy of paper tape in preventing hypertrophic scar formation in surgical incisions that transverse Langer's skin tension lines, Plast Reconstr Surg. 2005, 116, 1648-56. 
[74] F. RE., Treatment of inflamed hypertrophic scars using intralesional 5-FU, Dermatol Surg. 1999, 25, 224-32.

[75] L. O'Brien, A. Pandit, Silicon gel sheeting for preventing and treating hypertrophic and keloid scars, Cochrane Database Syst Rev. 2006, CD003826.

[76] P. Agbenorku, Triple keloid therapy: a combination of steroids, surgery and silicone gel strip/sheet for keloid treatment., Eur J Plast Surg. 2000, 23, 1501 .

[77] Q. Wang, H. Cheng, H. Peng, H. Zhou, P.Y. Li, R. Langer, Non-genetic engineering of cells for drug delivery and cell-based therapy, Adv Drug Deliv Rev. 2015, 91, 125-40.

[78] M.R. Prausnitz, R. Langer, Transdermal drug delivery, Nat Biotechnol. 2008, 26, 1261-8.

[79] J.W. Lee, M.R. Han, J.H. Park, Polymer microneedles for transdermal drug delivery, J Drug Target. 2013, 21, 211-23.

[80] H.P. Ehrlich, B. Sun, G.C. Saggers, F. Kromath, Gap junction communications influence upon fibroblast synthesis of Type I collagen and fibronectin, J Cell Biochem. 2006, 98, 735-43.

[81] C. Qiu, P. Coutinho, S. Frank, S. Franke, L.Y. Law, P. Martin, C.R. Green, D.L. Becker, Targeting connexin43 expression accelerates the rate of wound repair, Curr Biol. 2003, 13, 1697-703.

[82] P. Coutinho, C. Qiu, S. Frank, K. Tamber, D. Becker, Dynamic changes in connexin expression correlate with key events in the wound healing process, Cell Biol Int. 2003, 27, 525-41.

[83] R.G. Gourdie, G.S. Ghatnekar, M. O'Quinn, M.J. Rhett, R.J. Barker, C. Zhu, J. Jourdan, A.W. Hunter, The unstoppable connexin 43 carboxyl-terminus: new roles in gap junction organization and wound healing, Ann N Y Acad Sci. 2006, 1080, 49-62.

[84] J.M. Rhett, G.S. Ghatnekar, J.A. Palatinus, M. O'Quinn, M.J. Yost, R.G. Gourdie, Novel therapies for scar reduction and regenerative healing of skin wounds, Trends Biotechnol. 2008, 26, 173-80.

[85] P. Coutinho, C. Qiu, S. Frank, C.M. Wang, T. Brown, C.R. Green, D.L. Becker, Limiting burn extension by transient inhibition of Connexin43 expression at the site of injury, Br J Plast Surg. 2005, 58, 658-67.

[86] B. Cogliati, M. Vinken, T.C. Silva, C.M. Araujo, T.P. Aloia, L.M. Chaible, C.M. Mori, M.L. Dagli, Connexin 43 deficiency accelerates skin wound healing and extracellular matrix remodeling in mice, J Dermatol Sci. 2015, 79, 50-6.

[87] W.H. Evans, L. Leybaert, Mimetic peptides as blockers of connexin channel-facilitated intercellular communication, Cell Commun Adhes. 2007, 14, 265-73.

[88] P.C. Cheng, The contrast formation in optical microscopy, in: J.B. Pawley (Ed.), Handbook of Biological Confocal Microscopy, Springer US, New York, 2006, pp. 162-206.

[89] W.C.W. Chan, S. Nie, Quantum dot bioconjugates for ultrasensitive nonisotopic detection, Science. 1998, 281, 2016-8. 
[90] X. Wu, H. Liu, J. Liu, K.N. Haley, J.A. Treadway, J.P. Larson, N. Ge, F. Peale, M.P. Bruchez, Immunofluorescent labeling of cancer marker Her2 and other cellular targets with semiconductor quantum dots, Nat. Biotechnol. 2003, 21, 41-6.

[91] Q. Liu, B. Guo, Z. Rao, B. Zhang, J.R. Gong, Strong two-photon-induced fluorescence from photostable, biocompatible nitrogen-doped graphene quantum dots for cellular and deep-tissue imaging, Nano Lett. 2013, 13, 243641.

[92] L.A. Osminkina, K.P. Tamarov, A.P. Sviridov, R.A. Galkin, M.B. Gongalsky, V.V. Solovyev, A.A. Kudryavtsev, V.Y. Timoshenko, Photoluminescent biocompatible silicon nanoparticles for cancer theranostic applications, J Biophotonics. 2012, 5, 529-35.

[93] A. Burns, H. Ow, U. Wiesner, Fluorescent core-shell silica nanoparticles: towards "Lab on a Particle" architectures for nanobiotechnology, Chem Soc Rev. 2006, 35, 1028-42.

[94] W. Qin, K. Li, G. Feng, M. Li, Z. Yang, B. Liu, B.Z. Tang, Bright and Photostable Organic Fluorescent Dots with Aggregation-Induced Emission Characteristics for Noninvasive Long-Term Cell Imaging, Adv. Funct. Mater. 2013, 24, 635-43.

[95] N.C. Shaner, M.Z. Lin, M.R. McKeown, P.A. Steinbach, K.L. Hazelwood, M.W. Davidson, R.Y. Tsien, Improving the photostability of bright monomeric orange and red fluorescent proteins, Nat Methods. 2008, 5, 545-51.

[96] K. Li, W. Qin, D. Ding, N. Tomczak, J. Geng, R. Liu, J. Liu, X. Zhang, H. Liu, B. Liu, B.Z. Tang, Photostable fluorescent organic dots with aggregationinduced emission (AIE dots) for noninvasive long-term cell tracing, Sci Rep. 2013, 3, 1150.

[97] Q. Zhao, K. Li, S. Chen, A. Qin, D. Ding, S. Zhang, Y. Liu, B. Liu, J.Z. Sun, B.Z. Tang, Aggregation-induced red-NIR emission organic nanoparticles as effective and photostable fluorescent probes for bioimaging, J Mater Chem. 2012, 22, 15128-35.

[98] C.W.T. Leung, Y. Hong, S. Chen, E. Zhao, J.W.Y. Lam, B.Z. Tang, A photostable AIE luminogen for specific mitochondrial imaging and tracking, J Am Chem Soc. 2012, 135, 62-5.

[99] M.A. Woodruff, D.W. Hutmacher, The return of a forgotten polymerpolycaprolactone in the 21st century, Prog Polym Sci. 2010, 35, 1217-56.

[100] S. Kuddannaya, Y.J. Chuah, M.H.A. Lee, N.V. Menon, Y. Kang, Y. Zhang, Surface chemical modification of poly(dimethylsiloxane) for the enhanced adhesion and proliferation of mesenchymal stem cells, ACS Appl Mater Inter. 2013, 5, 9777-84.

[101] J.-S. Hu, Y. Guo, H.-P. Liang, L.-J. Wan, L. Jiang, Three-dimensional self-organization of supramolecular self-assembled porphyrin hollow hexagonal nanoprisms, J Am Chem Soc. 2005, 127, 17090-5.

[102] M. Akbulut, P. Ginart, M.E. Gindy, C. Theriault, K.H. Chin, W. Soboyejo, R.K. Prud'homme, Generic method of preparing multifunctional 
fluorescent nanoparticles using flash nanoPrecipitation, Adv Funct Mater. 2009, $19,718-25$.

[103] X. Zhang, C. Dong, J.A. Zapien, S. Ismathullakhan, Z. Kang, J. Jie, X. Zhang, J.C. Chang, C.-S. Lee, S.-T. Lee, Polyhedral organic microcrystals: from cubes to rhombic dodecahedra, Angew Chem Int Ed. 2009, 48, 9121-3.

[104] F. Bai, Z. Sun, H. Wu, R.E. Haddad, E.N. Coker, J.Y. Huang, M.A. Rodriguez, H. Fan, Porous one-dimensional nanostructures through confined cooperative self-assembly, Nano Lett. 2011, 11, 5196-200.

[105] L. Tang, X. Yang, Q. Yin, K. Cai, H. Wang, I. Chaudhury, C. Yao, Q. Zhou, M. Kwon, J.A. Hartman, I.T. Dobrucki, L.W. Dobrucki, L.B. Borst, S. Lezmi, W.G. Helferich, A.L. Ferguson, T.M. Fan, J. Cheng, Investigating the optimal size of anticancer nanomedicine, PNAS. 2014, 111, 15344-9.

[106] S. Huang, S.Y. Liu, K. Wang, C.J. Yang, Y.M. Luo, Y.D. Zhang, B. Cao, Y.J. Kang, M.F. Wang, Highly fluorescent and bioresorbable polymeric nanoparticles with enhanced photostability for cell imaging, Nanoscale. 2015, 7, 889-95.

[107] R.F. Kubin, A.N. Fletcher, Fluorescence quantum yields of some rhodamine dyes, J Lumin. 1982, 27, 455-62.

[108] L.Z. Chen, J.M. Zheng, Y. Zhang, L.X. Yang, J.Q. Wang, J. Ni, D.X. Cui, C.Q. Yu, Z.L. Cai, Tumor-specific expression of microRNA-26a suppresses human hepatocellular carcinoma growth via cyclin-dependent and independent pathways, Mol Ther. 2011, 19, 1521-8.

[109] G.K. Wilson, Z. Stamataki, In vitro systems for the study of hepatitis C virus infection, Int J Hepatol. 2012, 2012, 292591.

[110] T. Kato, M. Tanaka, M. Oba, Protein transfection study using multicellular tumor spheroids of human hepatoma Huh-7 Cells, PLoS One. 2013, 8 .

[111] K. Ma, H. Shen, S. Shen, M. Xie, C. Mao, L. Qiu, Y. Jin, Development of a successive targeting liposome with multi-ligand for efficient targeting gene delivery, J Gene Med. 2011, 13, 290-301.

[112] S.V. Boddapati, G.G.M. D’Souza, S. Erdogan, V.P. Torchilin, V. Weissig, Organelle-targeted nanocarriers: specific delivery of liposomal ceramide to mitochondria enhances Its cytotoxicity in vitro and in vivo, Nano Lett. 2008, 8, 2559-63.

[113] M.L. Barcellona, E. Gratton, The fluorescence properties of a DNA probe, Eur Biophys J. 1990, 17, 315-23.

[114] G. Cosa, K.S. Focsaneanu, J.R.N. McLean, J.P. McNamee, J.C. Scaiano, Photophysical properties of fluorescent DNA-dyes bound to single- and doublestranded DNA in aqueous buffered solution, Photochem Photobiol. 2001, 73, 585-99.

[115] Y. Gao, Y. Cui, J.K. Chan, C. Xu, Stem cell tracking with optically active nanoparticles, Am J Nucl Med Mol Imaging. 2013, 3, 232-46.

[116] V.F.M. Segers, R.T. Lee, Stem-cell therapy for cardiac disease, Nature. 2008, 451, 937-42. 
[117] O. Lindvall, Z. Kokaia, Stem cells for the treatment of neurological disorders, Nature. 2006, 441, 1094-6.

[118] C. Aguayo-Mazzucato, S. Bonner-Weir, Stem cell therapy for type 1 diabetes mellitus, Nat Rev Endocrinol. 2010, 6, 139-48.

[119] U. Noth, A.F. Steinert, R.S. Tuan, Technology Insight: adult mesenchymal stem cells for osteoarthritis therapy, Nat Clin Pract Rheumatol. 2008, 4, 371-80.

[120] X. Wei, X. Yang, Z.P. Han, F.F. Qu, L. Shao, Y.F. Shi, Mesenchymal stem cells: a new trend for cell therapy, Acta Pharmacol Sin. 2013, 34, 747-54. [121] S.H. Wang, X.B. Qu, R.C. Zhao, Clinical applications of mesenchymal stem cells, J Hematol Oncol. 2012, 5.

[122] M. Edmundson, N.T.K. Thanh, B. Song, Nanoparticles based stem cell tracking in regenerative medicine, Theranostics. 2013, 3, 573-82.

[123] A. Tautzenberger, A. Kovtun, A. Ignatius, Nanoparticles and their potential for application in bone, Int J Nanomedicine. 2012, 7, 4545-57.

[124] Y.C. Yu Gao, Jerry KY Chan, Chenjie Xu, Stem cell tracking with optically active nanoparticles, Am J Nucl Med Mol Imaging. 2013, 3, 232-46.

[125] B.S. Shah, P.A. Clark, E.K. Moioli, M.A. Stroscio, J.J. Mao, Labeling of mesenchymal stem cells by bioconjugated quantum dots, Nano Lett. 2007, 7, 3071-9.

[126] B.S. Shah, J.J. Mao, Labeling of mesenchymal stem cells with bioconjugated quantum dots, Methods Mol Biol. 2011, 680, 61-75.

[127] C.J. Xu, L.Y. Mu, I. Roes, D. Miranda-Nieves, M. Nahrendorf, J.A. Ankrum, W.A. Zhao, J.M. Karp, Nanoparticle-based monitoring of cell therapy, Nanotechnology. 2011, 22.

[128] C.J. Xu, W.A. Zhao, Nanoparticle-based monitoring of stem cell therapy, Theranostics. 2013, 3, 616-7.

[129] E.J. Sutton, T.D. Henning, B.J. Pichler, C. Bremer, H.E. Daldrup-Link, Cell tracking with optical imaging, Eur Radiol. 2008, 18, 2021-32.

[130] I.L. Medintz, H.T. Uyeda, E.R. Goldman, H. Mattoussi, Quantum dot bioconjugates for imaging, labelling and sensing, Nature Materials. 2005, 4, 435-46.

[131] K.M. Dupont, K. Sharma, H.Y. Stevens, J.D. Boerckel, A.J. Garcia, R.E. Guldberg, Human stem cell delivery for treatment of large segmental bone defects, Proc Natl Acad Sci U S A. 2010, 107, 3305-10.

[132] Y.Y. Su, Y. He, H.T. Lu, L.M. Sai, Q.N. Li, W.X. Li, L.H. Wang, P.P. Shen, Q. Huang, C.H. Fan, The cytotoxicity of cadmium based, aqueous phasesynthesized, quantum dots and its modulation by surface coating, Biomaterials. 2009, 30, 19-25.

[133] N. Chen, Y. He, Y.Y. Su, X.M. Li, Q. Huang, H.F. Wang, X.Z. Zhang, R.Z. Tai, C.H. Fan, The cytotoxicity of cadmium-based quantum dots, Biomaterials. 2012, 33, 1238-44.

[134] S.C. Hsieh, F.F. Wang, C.S. Lin, Y.J. Chen, S.C. Hung, Y.J. Wang, The inhibition of osteogenesis with human bone marrow mesenchymal stem cells by CdSe/ZnS quantum dot labels, Biomaterials. 2006, 27, 1656-64. 
[135] A. Tautzenberger, S. Lorenz, L. Kreja, A. Zeller, A. Musyanovych, H. Schrezenmeier, K. Landfester, V. Mailander, A. Ignatius, Effect of functionalised fluorescence-labelled nanoparticles on mesenchymal stem cell differentiation, Biomaterials. 2010, 31, 2064-71.

[136] Z. Yang, Y.N. Wu, C. Li, T.T. Zhang, Y. Zou, J.H.P. Hui, Z.G. Ge, E.H. Lee, Improved mesenchymal stem cells attachment and In vitro cartilage tissue formation on chitosan-modified poly(L-lactide-co-epsilon-caprolactone) scaffold, Tissue Eng Part A. 2012, 18, 242-51.

[137] Z. Yang, J.F. Schmitt, E.H. Lee, Immunohistochemical analysis of human mesenchymal stem cells differentiating into chondrogenic, osteogenic, and adipogenic lineages, Methods Mol Biol. 2011, 698, 353-66.

[138] T.D. Schmittgen, K.J. Livak, Analyzing real-time PCR data by the comparative C-T method, Nat Protoc. 2008, 3, 1101-8.

[139] S. Liu, L.M. Tay, R. Anggara, Y.J. Chuah, Y. Kang, Long-term tracking mesenchymal stem cell differentiation with photostable fluorescent nanoparticles, ACS Appl Mater Interfaces. 2016, 8, 11925-33.

[140] A.J. Sophia Fox, A. Bedi, S.A. Rodeo, The basic science of articular cartilage: structure, composition, and function, Sports Health. 2009, 1, 461-8.

[141] C. Karlsson, C. Brantsing, T. Svensson, H. Brisby, J. Asp, T. Tallheden, A. Lindahl, Differentiation of human mesenchymal stem cells and articular chondrocytes: Analysis of chondrogenic potential and expression pattern of differentiation-related transcription factors, J Orthop Res. 2007, 25, 152-63.

[142] Y.H. Hou, K.Y. Cai, J.H. Li, X.Y. Chen, M. Lai, Y. Hu, Z. Luo, X.W. Ding, D.W. Xu, Effects of titanium nanoparticles on adhesion, migration, proliferation, and differentiation of mesenchymal stem cells, Int $\mathrm{J}$ Nanomedicine. 2013, 8, 3619-30.

[143] H. Geckil, F. Xu, X.H. Zhang, S. Moon, U. Demirci, Engineering hydrogels as extracellular matrix mimics, Nanomedicine. 2010, 5, 469-84.

[144] M. Ravi, V. Paramesh, S.R. Kaviya, E. Anuradha, F.D.P. Solomon, 3D cell culture systems: advantages and applications, J Cell Physio. 2015, 230, 1626.

[145] V. Liu Tsang, S.N. Bhatia, Three-dimensional tissue fabrication, Adv Drug Deliv Rev. 2004, 56, 1635-47.

[146] I.H. Jaafar, C.E. LeBlon, M.-T. Wei, D. Ou-Yang, J.P. Coulter, S.S. Jedlicka, Improving fluorescence imaging of biological cells on biomedical polymers, Acta Biomater. 2011, 7, 1588-98.

[147] R.F. Donnelly, T.R. Raj Singh, A.D. Woolfson, Microneedle-based drug delivery systems: microfabrication, drug delivery, and safety, Drug Deliv. 2010, 17, 187-207.

[148] J. Yu, Y. Zhang, Y. Ye, R. DiSanto, W. Sun, D. Ranson, F.S. Ligler, J.B. Buse, Z. Gu, Microneedle-array patches loaded with hypoxia-sensitive vesicles provide fast glucose-responsive insulin delivery, Proc Natl Acad Sci U S A. $2015,112,8260-5$. 
[149] Y. Zhang, J. Yu, J. Wang, N.J. Hanne, Z. Cui, C. Qian, C. Wang, H. Xin, J.H. Cole, C.M. Gallippi, Y. Zhu, Z. Gu, Thrombin-responsive transcutaneous patch for auto-anticoagulant regulation, Adv Mater. 2017, 29, 1604043.

[150] J.W. Lee, M.R. Han, J.H. Park, Polymer microneedles for transdermal drug delivery, J Drug Target. 2012, 21, 211-23.

[151] J.H. Park, M.G. Allen, M.R. Prausnitz, Polymer microneedles for controlled-release drug delivery, Pharm Res. 2006, 23, 1008-19.

[152] M. Wang, L. Hu, C. Xu, Recent advances in the design of polymeric microneedles for transdermal drug delivery and biosensing, Lab Chip. 2017, 17, 1373-87.

[153] C.A. Durst, M.P. Cuchiara, E.G. Mansfield, J.L. West, K.J. Grande-Allen, Flexural characterization of cell encapsulated PEGDA hydrogels with applications for tissue engineered heart valves, Acta Biomater. 2011, 7, 246776.

[154] J. Jia, R.C. Coyle, D.J. Richards, C.L. Berry, R.W. Barrs, J. Biggs, C. James Chou, T.C. Trusk, Y. Mei, Development of peptide-functionalized synthetic hydrogel microarrays for stem cell and tissue engineering applications, Acta Biomater. 2016, 45, 110-20.

[155] A. Sabnis, M. Rahimi, C. Chapman, K.T. Nguyen, Cytocompatibility studies of an in situ photopolymerized thermoresponsive hydrogel nanoparticle system using human aortic smooth muscle cells, J Biomed Mater Res A. 2009, 91, 52-9.

[156] S. Lee, X. Tong, F. Yang, The effects of varying poly(ethylene glycol) hydrogel crosslinking density and the crosslinking mechanism on protein accumulation in three-dimensional hydrogels, Acta Biomater. 2014, 10, 416774.

[157] H.S. Gill, M.R. Prausnitz, Coating formulations for microneedles, Pharm Res. 2007, 24, 1369-80.

[158] P. Xue, D.C.L. Yeo, Y.J. Chuah, H.L. Tey, Y. Kang, C. Xu, Drug-eluting microneedles for self-administered treatment of keloids, Technology. 2014, 02, 144-52.

[159] A.A. Ali, C.M. McCrudden, J. McCaffrey, J.W. McBride, G. Cole, N.J. Dunne, T. Robson, A. Kissenpfennig, R.F. Donnelly, H.O. McCarthy, DNA vaccination for cervical cancer; a novel technology platform of RALA mediated gene delivery via polymeric microneedles, Nanomedicine. 2016, 13, 921-32.

[160] P.C. DeMuth, X. Su, R.E. Samuel, P.T. Hammond, D.J. Irvine, Nanolayered microneedles for transcutaneous delivery of polymer nanoparticles and plasmid DNA, Adv Mater. 2010, 22, 4851-6.

[161] N.W. Kim, M.S. Lee, K.R. Kim, J.E. Lee, K. Lee, J.S. Park, Y. Matsumoto, D.G. Jo, H. Lee, D.S. Lee, J.H. Jeong, Polyplex-releasing microneedles for enhanced cutaneous delivery of DNA vaccine, J Control Release. 2014, 179, 11-7.

[162] Y.C. Kim, D.G. Yoo, R.W. Compans, S.M. Kang, M.R. Prausnitz, Crossprotection by co-immunization with influenza hemagglutinin DNA and 
inactivated virus vaccine using coated microneedles, J Control Release. 2013, 172, 579-88.

[163] M. Burke, L. Augenstein, A comparison of the effects of ultraviolet and ionizing radiations on trypsin activity and on its constituent amino acids, Biochem J. 1969, 114, 535-45.

[164] M. Correia, M.T. Neves-Petersen, P.B. Jeppesen, S. Gregersen, S.B. Petersen, UV-light exposure of insulin: pharmaceutical implications upon covalent insulin dityrosine dimerization and disulphide bond photolysis, PLoS One. 2012, 7, e50733.

[165] D.L. Becker, C. Thrasivoulou, A.R. Phillips, Connexins in wound healing; perspectives in diabetic patients, Biochim Biophys Acta. 2012, 1818, 2068-75.

[166] M. Kretz, C. Euwens, S. Hombach, D. Eckardt, B. Teubner, O. Traub, K. Willecke, T. Ott, Altered connexin expression and wound healing in the epidermis of connexin-deficient mice, J Cell Sci. 2003, 116, 3443-52.

[167] E.L. Ongstad, M.P. O'Quinn, G.S. Ghatnekar, M.J. Yost, R.G. Gourdie, A connexin43 mimetic peptide promotes regenerative healing and improves mechanical properties in skin and heart, Adv Wound Care (New Rochelle). 2013, 2, 55-62.

[168] Shiying Liu, David C. Yeo, Christian Wiraja, Hong Liang Tey, Milan Mrksich, C. Xu, Peptide delivery with poly(ethylene glycol) diacrylate microneedles through swelling effect, Bioengineering \& Transla Med 2017, 110.

[169] A. Cavallo, M. Madaghiele, U. Masullo, M.G. Lionetto, A. Sannino, Photo-crosslinked poly(ethylene glycol) diacrylate (PEGDA) hydrogels from low molecular weight prepolymer: Swelling and permeation studies, J Appl Polym Sci. 2017, 134, 44380.

[170] B.J. Glass, R.G. Hu, A.R. Phillips, D.L. Becker, The action of mimetic peptides on connexins protects fibroblasts from the negative effects of ischemia reperfusion, Biol Open. 2015, 4, 1473-80.

[171] J.J. Shaffer, S.C. Taylor, F. Cook-Bolden, Keloidal scars: a review with a critical look at therapeutic options, J Am Acad Dermatol. 2002, 46, S63-S97.

[172] S.A. Shaker, N.N. Ayuob, N.H. Hajrah, Cell talk: a phenomenon observed in the keloid scar by immunohistochemical study, Appl Immunohistochem Mol Morphol. 2011, 19, 153-9.

[173] V. Hagel, T. Haraszti, H. Boehm, Diffusion and interaction in PEGDA hydrogels, Biointerphases. 2013, 8, 36-45.

[174] S. Lee, X. Tong, F. Yang, Effects of the poly(ethylene glycol) hydrogel crosslinking mechanism on protein release, Biomater Sci. 2016, 4, 405-11.

[175] S.P. Zustiak, H. Boukari, J.B. Leach, Solute diffusion and interactions in cross-linked poly(ethylene glycol) hydrogels studied by fluorescence correlation spectroscopy, Soft Matter. 2010, 6, 3609-18.

[176] L.M. Weber, C.G. Lopez, K.S. Anseth, Effects of PEG hydrogel crosslinking density on protein diffusion and encapsulated islet survival and function, J Biomed Mater Res A. 2009, 90, 720-9. 
[177] K. Moore, Z. Bryant, A. Vandergriff, G. Ghatnekar, R. Gourdie, J. Potts, Delivery of a novel connexin-43 mimetic peptide enhances wound healing, Microsc Microanal. 2013, 19, 216-7. 